\title{
DEVELOPMENT OF ELECTROSTATIC ACTUATORS WITH LARGE OUT-OF-PLANE DEFLECTION AND ITS APPLICATION IN SCANNING DISPLAY
}

\author{
By \\ Chao Fan \\ BSc, Electrical Engineering, University of Science and Technology Beijing, 2009 \\ MASc, Mechanical Engineering, Ryerson University, 2011 \\ A dissertation \\ presented to Ryerson University \\ in partial fulfillment of the \\ requirements for the degree of \\ Doctor of Philosophy \\ in the Program of \\ Mechanical and Industrial Engineering
}

Toronto, Ontario, Canada, 2016

(C) Fan Chao 2016 


\section{Author's Declaration}

I hereby declare that I am the sole author of this dissertation. This is a true copy of the dissertation, including any required final revisions, as accepted by my examiners.

I authorize Ryerson University to lend this dissertation to other institutions or individuals for the purpose of scholarly research.

I further authorize Ryerson University to reproduce this dissertation by photocopying or by other means, in total or in part, at the request of other institutions or individuals for the purpose of scholarly research.

I understand that my dissertation may be made electronically available to the public. 


\title{
Development of electrostatic actuators with large out-of-plane deflection and its application in scanning display
}

\section{Chao Fan}

\section{Doctor of Philosophy}

\section{Mechanical and Industrial Engineering, Ryerson University, 2016}

\begin{abstract}
Electrostatic out-of-plane microactuators have been widely used in applications of variable capacitors, optical attenuators, optical switches and scanning displays due to their small size, low cost, simple and diverse structure, low power consumption and high compatibility with semiconductor process. The large out-of-plane displacement of the microactuator with high reliability is preferred in order to increase the tuning range, tunability and the display size. However, the "pull-in" instability associated with conventional attractive-force electrostatic microactuators significantly limits the out-of-plane displacement and lowers the operation stability. A repulsive-force microactuator has been previously developed which can achieve large out-ofplane rotation and does not suffer from the "pull-in" instability. However, a larger rotation angle of the repulsive-force actuator is highly desired in order to improve its performance in the applications such as increasing the tunability and the scanning angle. In this thesis two novel repulsive-force actuators, i.e., two-row interdigitating-finger and two-width-finger (TWF) actuators are developed which output much larger out-of-plane rotation than the previous repulsive-force actuator without suffering from the "pull-in" instability. The mathematical models are established for both actuators using a hybrid approach. The actuators require only two thin layers and are suitable for surface micromachining process. The measured results show that the
\end{abstract}


two microactuators can achieve rotation angles of $11.5^{\circ}$ and $7.5^{\circ}$ at $150 \mathrm{~V}$ respectively. The improvements are $100 \%$ and $35 \%$ in comparison to the previous repulsive-force actuator with the same size, stiffness and driving voltage. A 2D scanning micromirror has been developed and fabricated based on the two-row-finger (TRF) actuator. Experimental results show the micromirror has larger rotation angle and faster response speed than those of the micromirror driven by the previous repulsive-force microactuator. The vector scanning display based on the micromirror is demonstrated. An advanced display approach is developed to generate displays with less distortion and higher refreshing rate compared to the previous generic display approach. The automotive Head-up Display (HUD) based on the micromirror and advanced display approach has been constructed for both real and virtual image configurations, which has advantages of small size, low cost, large viewing angle and good visibility over those HUDs in the market. 


\section{Acknowledgements}

I would like to thank:

* My parents for their love and countless sacrifices

My girlfriend, Shena, for her immense patience, emotional support and sacrifices.

My supervisor, Dr. Siyuan He, for his encouragement, support and guidance in this research project

* Tim, Terry and Auguest for being great graduate friends throughout my PhD time at Ryerson University

* The following groups for their collaboration and financial support on this project:

- Ryerson University

- CMC Microsystems 


\section{Dedication}

This dissertation is dedicated to my father, mother, grandparents and my girlfriend. 


\section{Table of Contents}

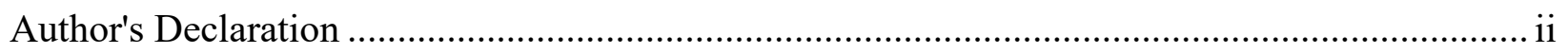

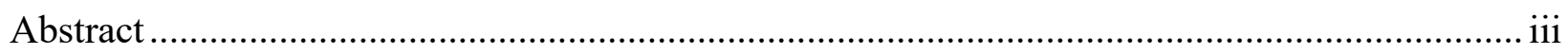

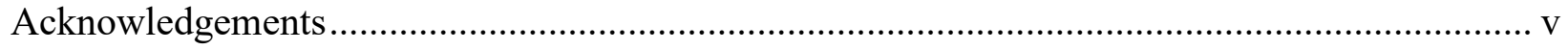

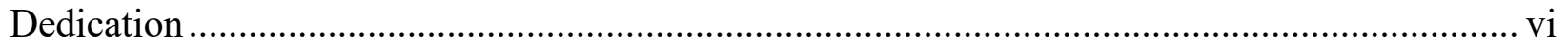

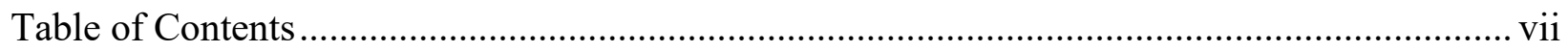

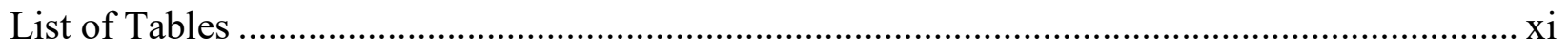

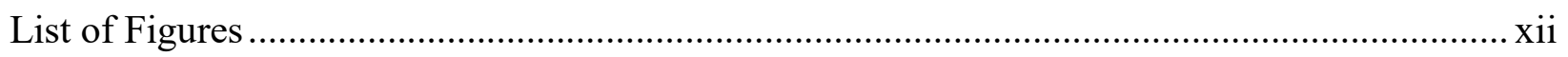

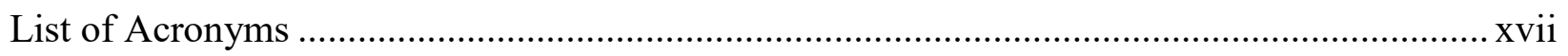

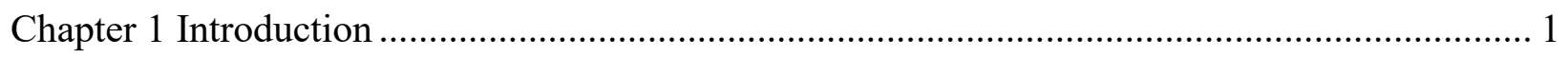

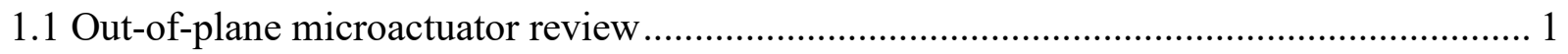

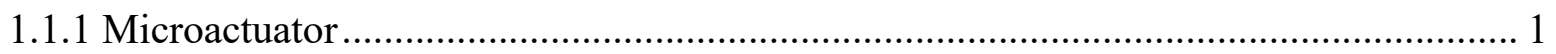

1.1.2 Electrostatic attractive-force out-of-plane rotation actuators ...................................... 2

1.1.3 Electrostatic repulsive-force out-of-plane actuators ................................................. 8

1.2 Micromirror based laser scanning display review ........................................................ 13

1.3 Automotive head-up display (HUD) review ............................................................... 17

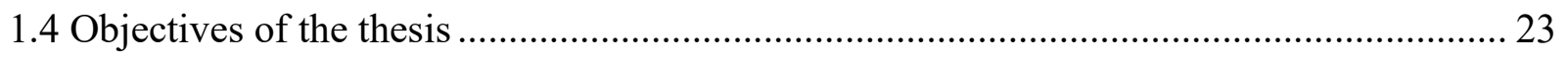

Chapter 2 Two-row interdigitating-finger repulsive-torque electrostatic actuator ........................ 25

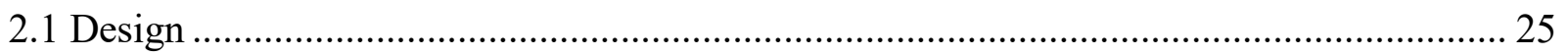

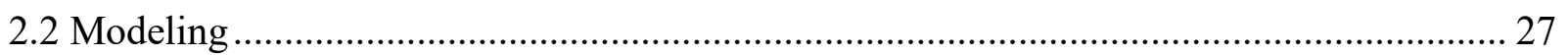

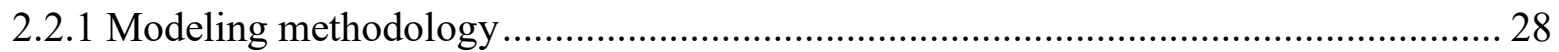

2.2.2 Modeling ........................................................................................................... 29

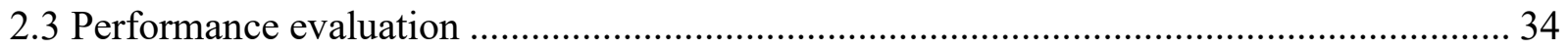

2.3.1 Rotation angle estimation and comparison with one-row-finger (ORF) actuator ....... 34

2.3.2 Dynamic response estimation by numerical simulation ............................................. 37 
2.4 Prototype and performance testing ……........................................................................ 39

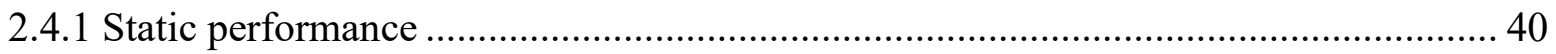

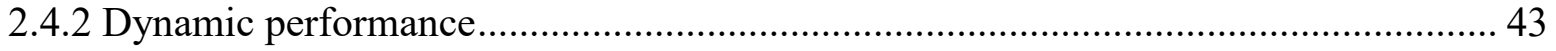

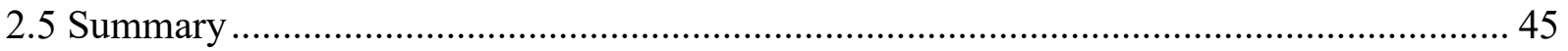

Chapter 3 Two-width-finger repulsive-torque electrostatic actuator.......................................... 47

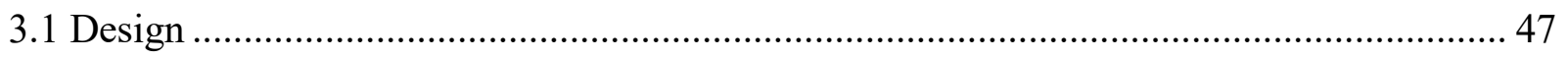

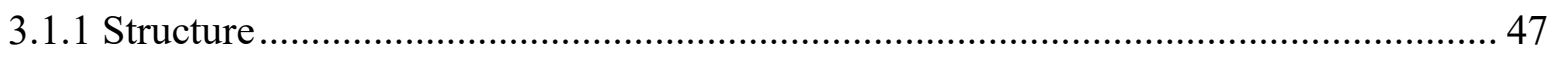

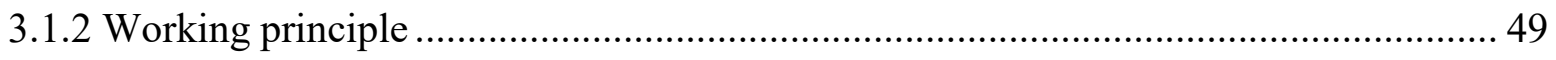

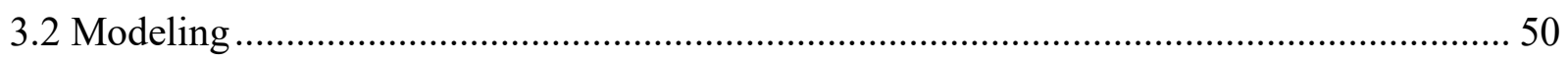

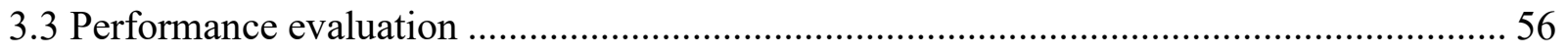

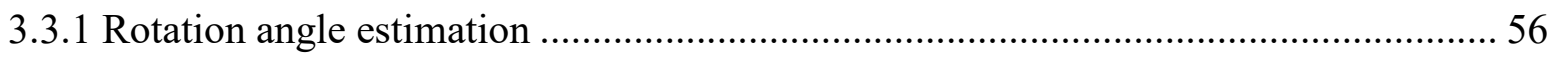

3.3.2 Dynamic response estimation ............................................................................. 57

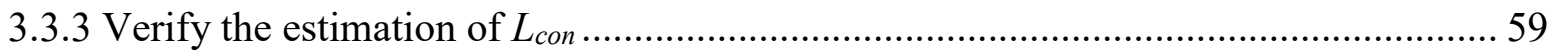

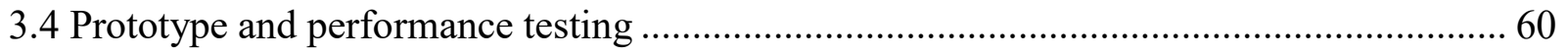

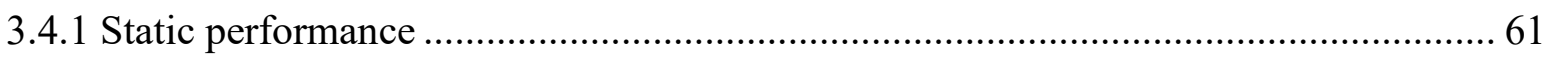

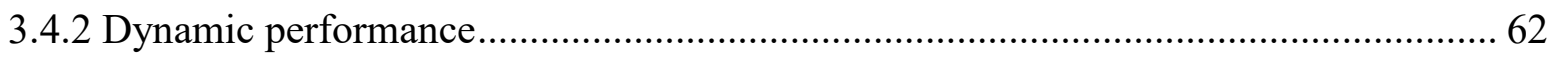

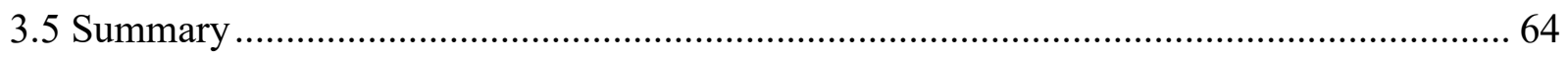

Chapter 4 Micromirror driven by the two-row interdigitating-finger actuator for vector display 65

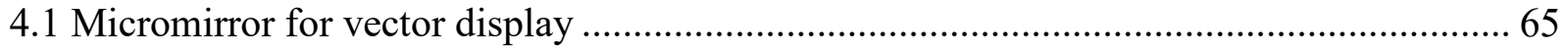

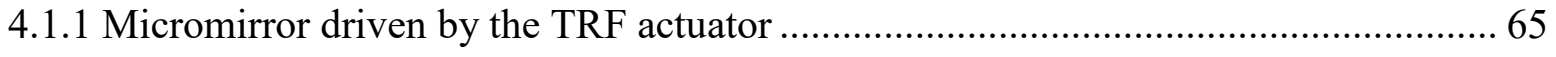

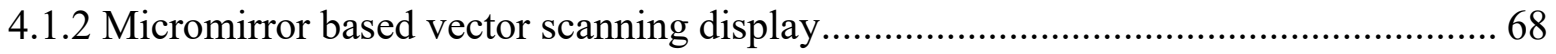

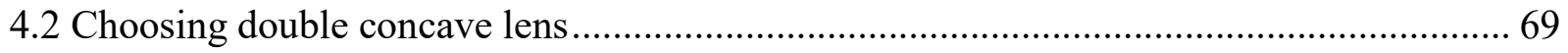

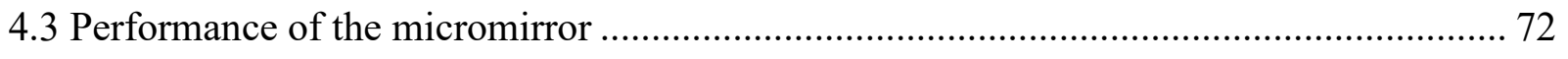

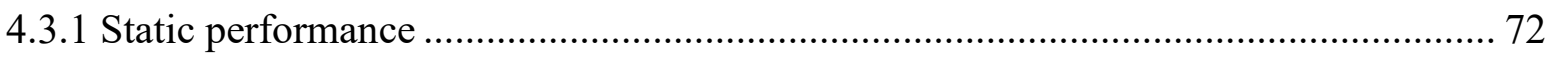

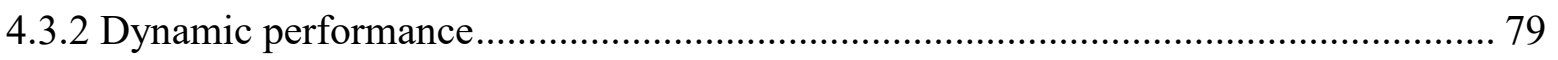




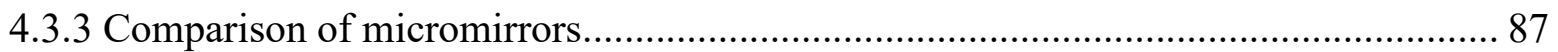

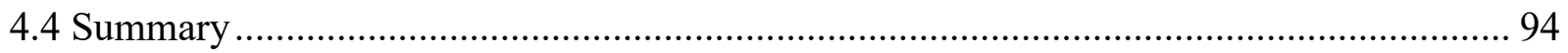

Chapter 5 Advanced display approach for micromirror based vector display............................... 96

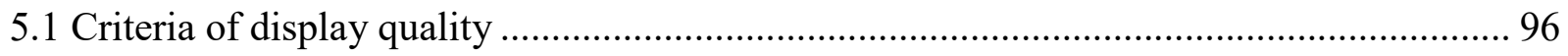

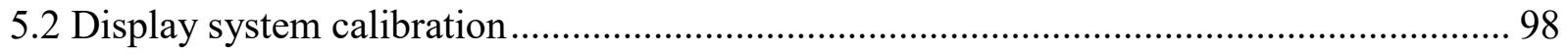

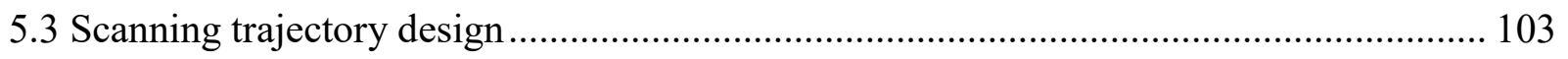

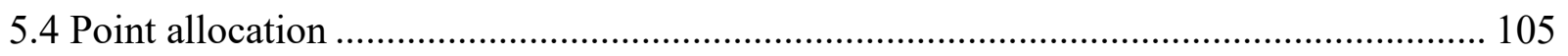

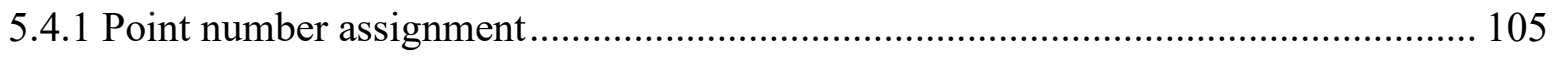

5.4.2 Design point distribution .................................................................................... 110

5.5 Control voltage generation and parameter identification............................................... 117

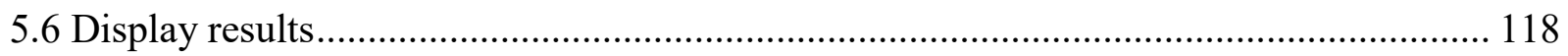

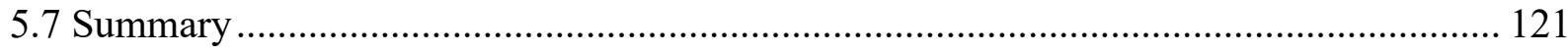

Chapter 6 Application of the micromirror on automotive head-up display (HUD) ................... 123

6.1 Design and working principle ..................................................................................... 123

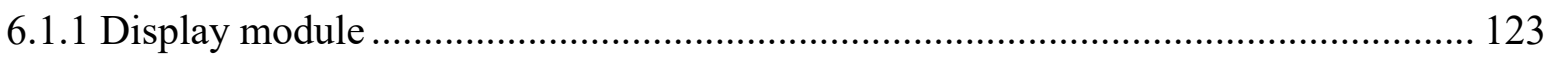

6.1.2 Real image and virtual image configuration of automotive HUD ............................ 125

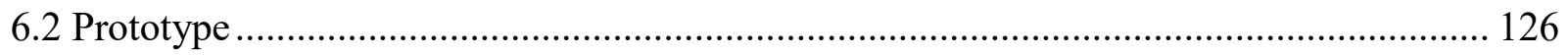

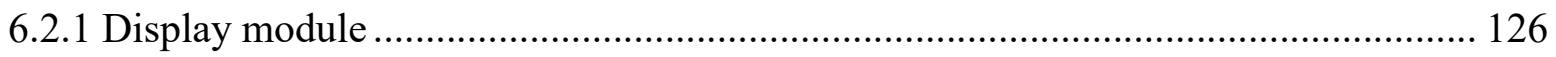

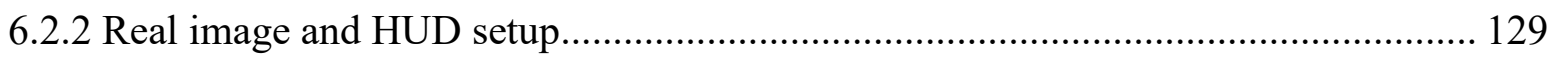

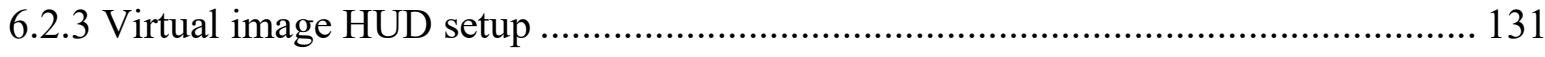

6.3 Compact design of display module ............................................................................. 135

6.4 Summary and comparison with the other HUDs ........................................................... 139

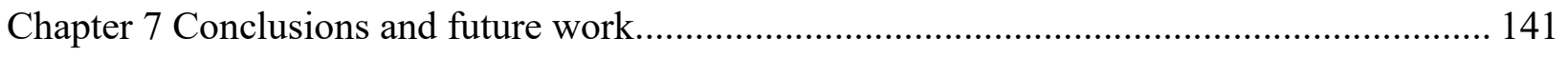

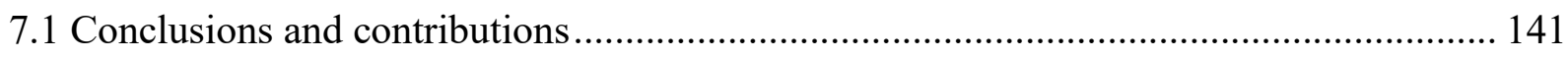

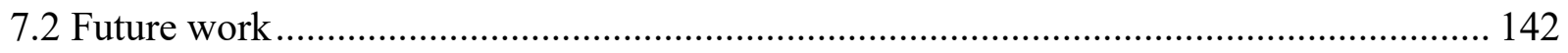




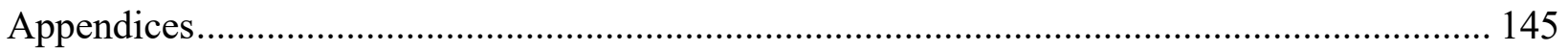

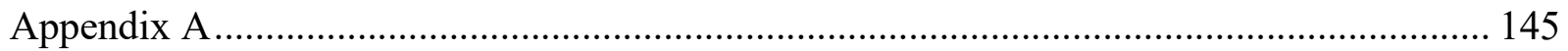

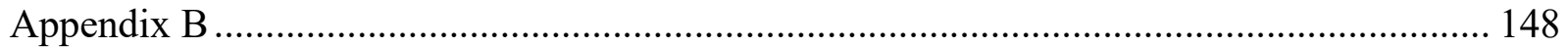

Appendix C Optical optimization results........................................................................ 148

Appendix D Bonding process of the protective glass .................................................. 149

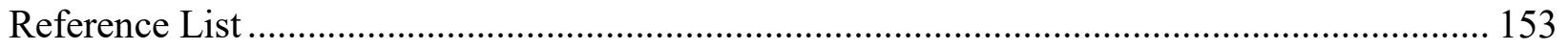




\section{List of Tables}

Table 3.1 Parameters of two-width finger actuator (unit: micrometer) ................................... 51

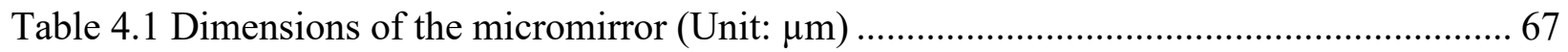

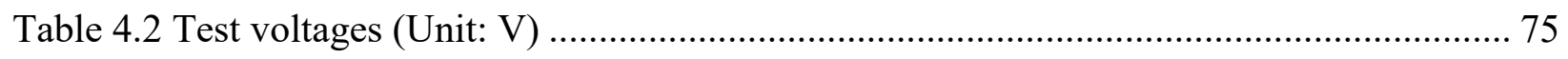

Table 4.3 Voltage combinations for step response test....................................................... 79

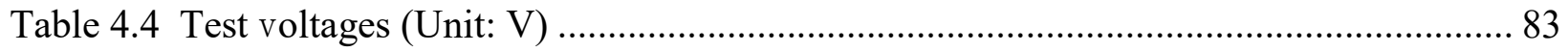

Table 5.1 The optimal parameters obtained using tral and error methods............................... 118 


\section{List of Figures}

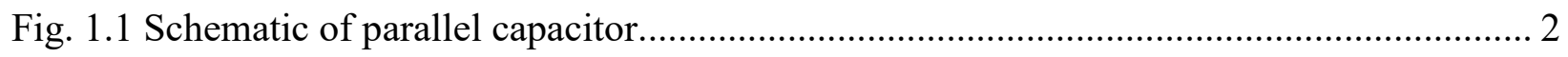

Fig. 1.2 Schematic view of the MEMS variable capacitor and SEM photographs of the fabricated

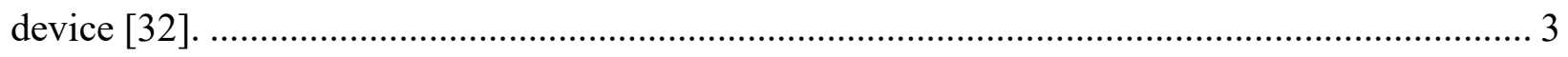

Fig. 1.3 A voltage controlled C-V response tuning and SEM image of the fabricated device [34].

Fig. 1.4 Basic structures of offset-drive, parallel plates and comb-drive [35].............................. 4

Fig. 1.5 Left: The schematic structure of the proposed micromirror. Right: The SEM micrograph of the fabricated micromirror with the other half of the micromirror removed [50]..................... 6

Fig. 1.6 (a) Comb-drive with fixed combs in one side of the movable comb (b) Dual mode comb-

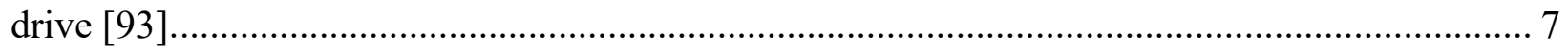

Fig. 1.7 Left: Schematic of 3-layer repulsive-force actuator. Right: Scanning electron microscope image $($ SEM) of a fabricated device with $100 \times 100 \mu$ m paddle [99] ...................................... 9

Fig. 1.8 The novel translation micromirror for adaptive optics [101] ....................................... 10

Fig. 1.9 SEM image of the optimized actuator fabricated using the PolyMUMPs process [105].11

Fig. 1.10 SEM images of the actuator subject to different voltages [107] .................................. 11

Fig. 1.11 Left: Galvanometer scanner and its driving circuit [113] Right: 2 axis galvanometer

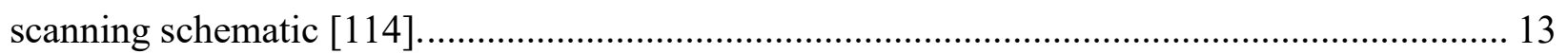

Fig. 1.12 (a) Laser projection based assembly and tooling guidance [115] (b) Laser show [116].

Fig. 1.13 Display results of laser raster scanning display based on electrostatic micromirrors [26], [93]. 15

Fig. 1.14 Vector scanning graphics using 2-layer surface micromachined micromirror [108] ... 15 Fig. 1.15 (a) Micromirror with vertical comb-drive (b) Vector scanning display (c) Raster scanning

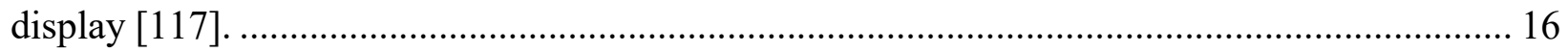

Fig. 1.16 A schematic view of the conventional HUD setup [126] ........................................... 18

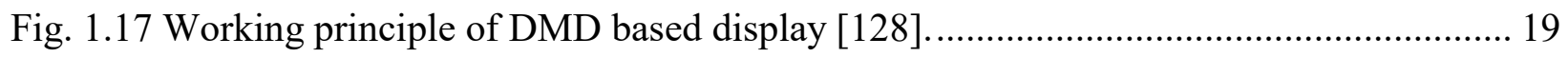

Fig. 1.18 Display result of LCoS based HUD in Land Rover Evoque [133]. .............................. 20

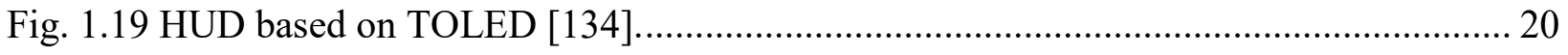

Fig. 1.20 (a) Electromagnetic resonant micromirror [135] (b) HUD based on the micromirror [135]. 
Fig. 1.21 HUD based on the micromirror [122]. 21

Fig. 1.22 (a) Micromirror fabricated using surface micromachining process [108] (b) HUD display result using the micromirror [109]. 23

Fig. 2.1 Two-row interdigitating-finger repulsive-torque electrostatic actuator. (a) Actuator at rest.

(b) Actuator in operation. 27

Fig. 2.2 Side view of interdigitating-fingers of lower and upper rows. (a) Actuator at rest position.

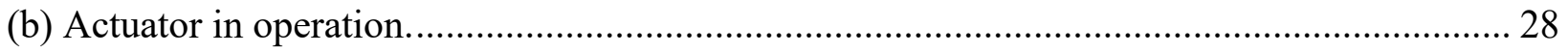

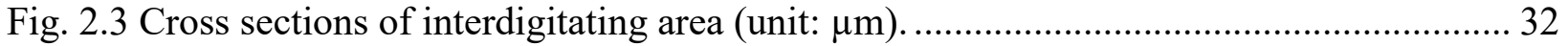
Fig. 2.4 Simulated and fitting results of electrostatic force in cross sections in the interdigitating

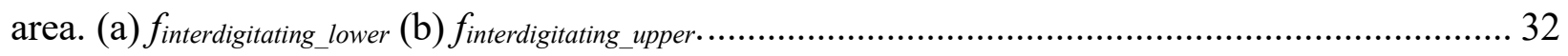
Fig. 2.5 Cross sections of non-interdigitating area (unit: $\mu \mathrm{m}$ ) …............................................... 32 Fig. 2.6 Simulated and fitting forces in cross sections of non-interdigitating area. .................... 33

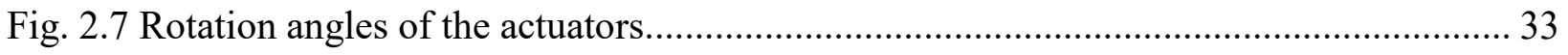

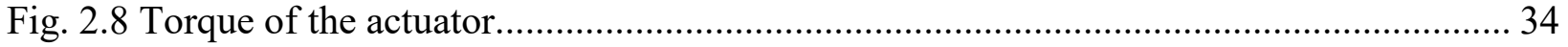

Fig. 2.9 Torque and rotation angle of the upper rotation fingers.............................................. 35

Fig. 2.10 Rotation angles of TRF actuator and ORF actuator at various actuator lengths........... 37

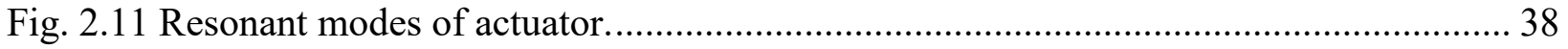

Fig. 2.12 SEM images of the prototype. (a) The actuator (b) Lower spring, lower and upper fingers. 39

Fig. 2.13 Experiment setup for measuring the rotation of the actuator. ……….......................... 39

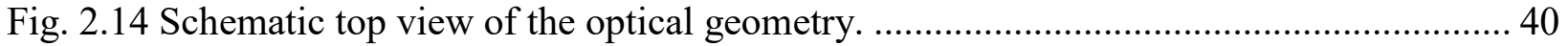

Fig. 2.15 Schematic side view of verifying the angle between the incident laser beam and the

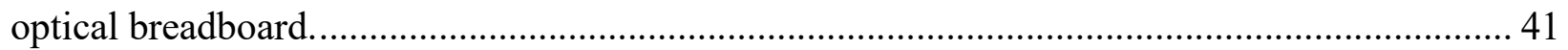

Fig. 2.16 Schematic top view of the geometry relationship between the tilt angle of the mirror plate and the displacement of the laser spot on the screen. ................................................................... 42

Fig. 2.17 Frequency response of the actuator (a) Magnitude response (b) Phase angle response.44 Fig. 3.1 Two-width finger actuator. (a) The actuator is at initial position. (b) The actuator is in

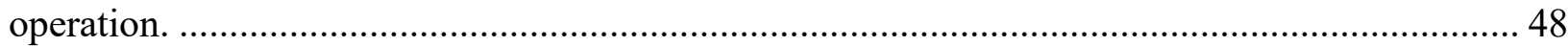

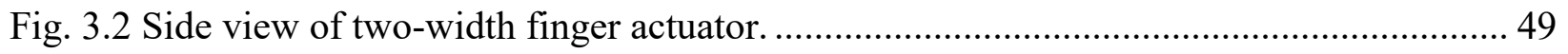

Fig. 3.3 (a) Top View of the actuator. (b) Enlarged view of part of the middle part.................... 51

Fig. 3.4 Simulation of rotation stiffness about Y-axis (see Fig. 3.1(b)) ....................................... 53 
Fig. 3.5 Mesh size convergence study. 53

Fig. 3.6 Schematic of cross sections. (a) Part of the lower part (b) Half of the middle part (c) Part of the upper part. 55

Fig. 3.7 Fitting curves and simulation data. 56 Fig. 3.8 (a) Modeling and experimental results (b) Percentage of the improvement of the TWF actuator over the previously developed ORF actuator. 58

Fig. 3.9 First mode deformation of the two-width finger actuator. 58 Fig. 3.10 Calculated rotation angles when $L_{C o n}$ is set to the length of the 4 fingers in the center and 6 finger on the side separately. 59

Fig. 3.11 SEM image of the TWF actuator (1) Overall view (2) Zoomed in view. ................... 60

Fig. 3.12 Experiment setup for measuring the rotation of the actuator. 61

Fig. 3.13 Bode plot of the two-width finger actuator (a) Magnitude response (b) Phase angle response....... 62

Fig. 3.14 Step response of the actuator from 0 to $5^{\circ}$ for a driving voltage of $76 \mathrm{~V}$. 63

Fig. 4.1 2D scanning micromirror based on TRF actuator. 67

Fig. 4.2 The prototype of the micromirror for vector scanning display. (a) SEM picture of the micromirror (b) Micromirror packaged in CQFP44 chip with a protective glass bonded on the top. 68

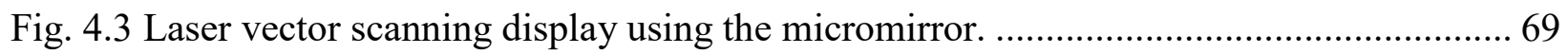

Fig. 4.4 Zemax model of the display system. ................................................................ 70

Fig. 4.5 Profile measure of the micromirror plate. ........................................................... 70

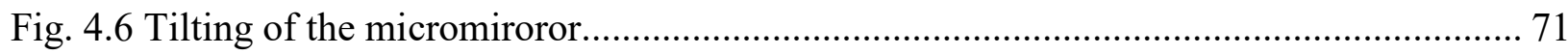

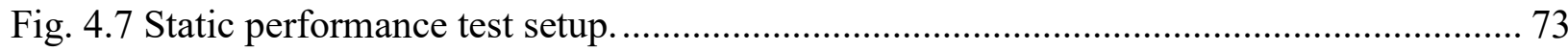

Fig. 4.8 Three driving approaches of the micromirror...................................................... 73

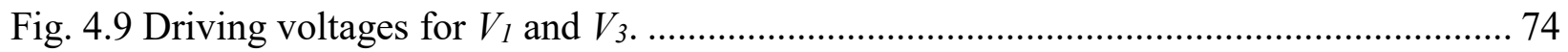

Fig. 4.10 Error introduced by manually marking. ........................................................... 77

Fig. 4.11 Micromirror rotation angles under different base voltages. .................................. 78

Fig. 4.12 Mechanical rotation of the micromirror. .............................................................. 78

Fig. 4.13 Rise time of the micromirror under different base voltages.................................. 81

Fig. 4.14 Percentage of overshoot of the micromirror under different base voltages. ............... 81

Fig. 4.15 Settling time of the micromirror under different base voltages............................... 82 
Fig. 4.16 Bode plot for test 1 and 4 (a) Amplitude response (b) Phase response...................... 84

Fig. 4.17 Bode plot for test 2 and 5 (a) Amplitude response (b) Phase response...................... 85

Fig. 4.18 Bode plot for test 3 and 6 (a) Amplitude response (b) Phase response...................... 86

Fig. 4.19 Micromirror rotation angle comparison. ............................................................. 88

Fig. 4.20 Rise time comparisons of the micromirrors. ........................................................ 89

Fig. 4.21 (a) Response speed compassion of the micromirrors (b) Percentage of the improvement of the response speed of the TRF micromirror compared to the ORF micromirror................... 90

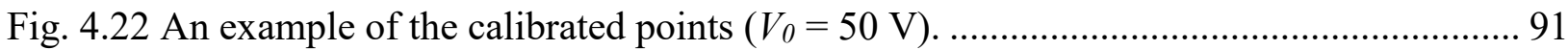
Fig. 4.23 Display results of the micromirrors $(30 \mathrm{~Hz})$ (a) ORF micromirror (b) TRF micromirror display: same size as the display of the ORF micromirror (c) TRF micromirror display: 1.7 times of the size of the ORF micromirror's display (d) TRF micromirror display: 2.8 times of the size of the ORF micromirror's display (e) TRF micromirror display: 4 times of the size of the ORF micromirror's display (f) TRF micromirror display: 5.8 times of the size of the ORF micromirror's

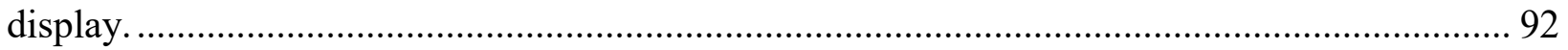
Fig. 4.24 Enlarged views of the displays in Fig. 4.23 (a) Enlarged view of Fig. 4.23 (a). (b)

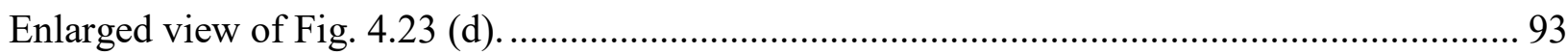

Fig. 4.25 Display results in 40Hz (a) ORF micromirror (b) TRF micromirror ......................... 94 Fig. 4.26 Display results of ORF and TRF micromirror in $40 \mathrm{~Hz}$ (a) ORF micromirror (b) TRF

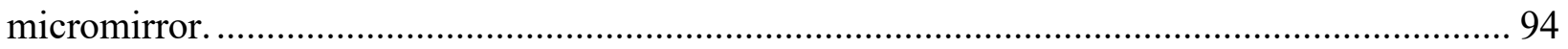

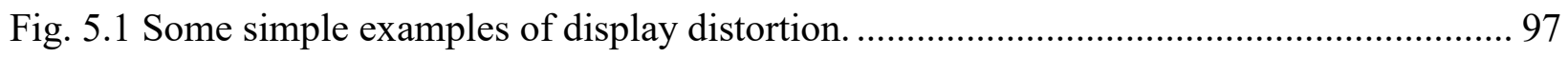

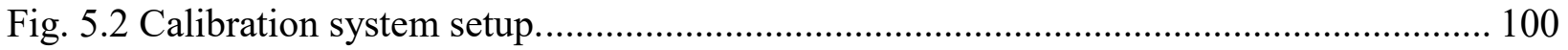

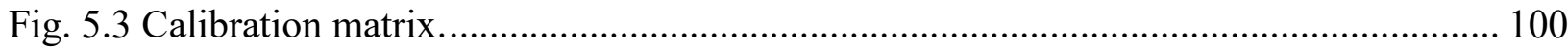

Fig. 5.4 Calibration RGB image split by 3 color channels (a) Green Channel image (b) Red channel

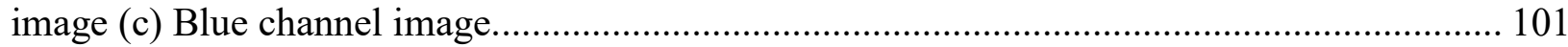

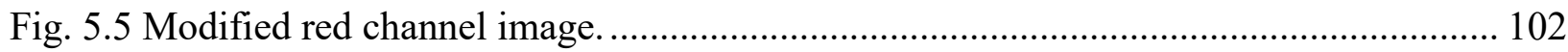

Fig. 5.6 Binary image converted from the modified red channel image. .............................. 102

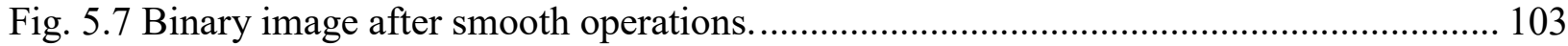

Fig. 5.8 Designed trajectory inside the calibration area.................................................. 103

Fig. 5.9 (a) Desired vector pattern (b) Visible and transitional paths of the desired vector pattern. 104 
Fig. 5.10 (a) Length level classification of the line segments (b) Length level classification of the transitional segments. 107

Fig. 5.11 One example of the distribution function expressed in Eq. (5.1) 109

Fig. 5.12 Display distortion with the increase of the refreshing rate (a) $30 \mathrm{~Hz}$ (b) $40 \mathrm{~Hz}$ (c) $50 \mathrm{~Hz}$.

Fig. 5.13 Point distribution on visible line segment. 112

Fig. 5.14 Point distribution on transitional segment. 114

Fig. 5.15 (a) $\sim$ (c)Two intersected segments with intersection angles $30^{\circ}, 90^{\circ}$, and $120^{\circ}$ respectively (d) Velocity variation from $V_{B}$ to $V_{B}{ }^{\prime}$ of the three cases illustrated in (a) - (c)...... 116

Fig. 5.16 Display setup. 117

Fig. 5.17 Display in $30 \mathrm{~Hz}$ (a) Advanced display approach (b) Generic display approach........ 119

Fig. 5.18 Display in $40 \mathrm{~Hz}$ (a) Advanced display approach (b) Generic display approach........ 119

Fig. 5.19 Display in $50 \mathrm{~Hz}$ (a) Advanced display approach (b) Generic display approach........ 119 Fig. 5.20 Display results of the ORF micromirror in $43 \mathrm{~Hz}$. Left: Generic display approach. Right: Advanced display approach. 121

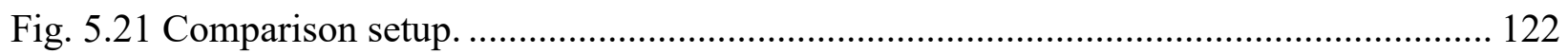

Fig. 5.22 Display result comparison using two micromirrors. .................................................. 122

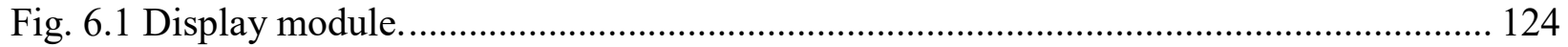

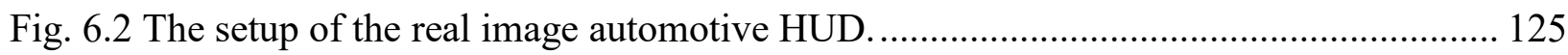

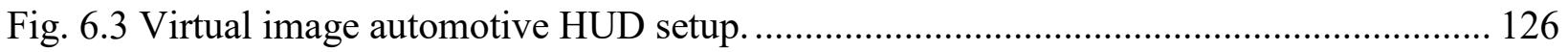

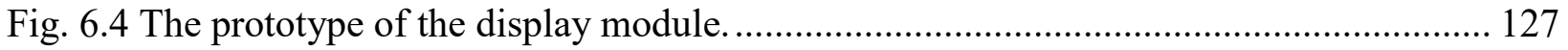

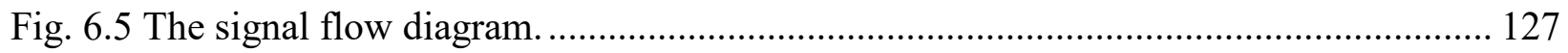

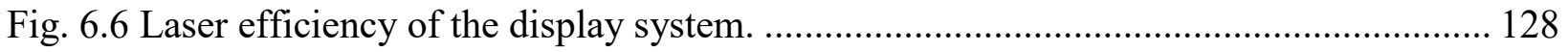

Fig. 6.7 (a) The reflected laser beam by the micromirror (b) The reflected laser beam after the

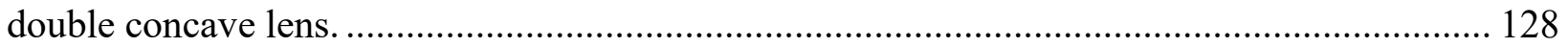

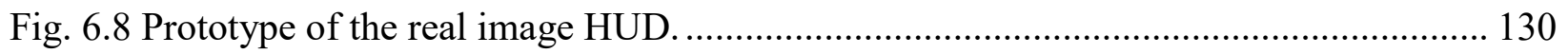

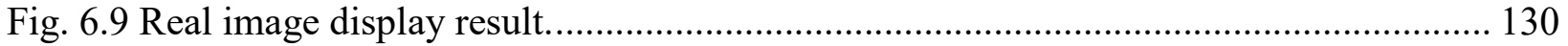

Fig. 6.10 Display films (a) Mirror-reflective film (b) Diffuse-reflective film (c) Diffuse-reflective film attacked on top of the mirror-reflective film.................................................................. 131

Fig. 6.11 The prototype of the virtual image HUD.............................................................. 132

Fig. 6.12 The projection screen used for virtual HUD. ......................................................... 132 


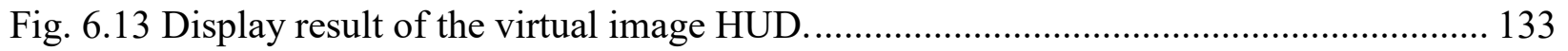

Fig. 6.14 (a) Micromirror vibration test system (b) Setup on the shaker............................... 134

Fig. 6.15 Compact structure design of the micromirror.................................................. 137

Fig. 6.16 (a) Prototype of the compact display module. (b) Display result of display module.. 137

Fig. 6.17 Real image HUD of compact display module................................................... 138

Fig. 6.18 Comparison of the diffuse-reflective films..................................................... 138

Fig. 6.19 Visibility test setup (a) Front view (b) Side view................................................ 139

Fig. 6.20 Display results under different external illumination (a) 2300 Lux (b) 6800 Lux ...... 139 


\section{List of Acronyms}

AVC Angular vertical comb-drive

DMD Digital micromirror device

HUD Head-up display

LSD Laser scanning display

LCoS Liquid crystal on silicon

MEMS Microelectromechanical system

MUMPs Multi-user MEMS process

OPD Optical path distance

ORF One-row-finger actuator

PSD Position sensing detector

PolyMUMPs Polysilicon multi-user MEMS process

SEM Scanning electron microscope

SOI Silicon on insulator

SVC Staggered vertical comb-drive

TRF Two-row interdigitating-finger actuator

TWF Two-width-finger actuator

XGA Extended graphics array 


\section{Chapter 1 Introduction}

\subsection{Out-of-plane microactuator review}

\subsubsection{Microactuator}

Microactuator is a microscopic mechanism that provides actuation motion to other micro structures. It is a constitutional device of Microelectromechanical Systems (MEMS). If classified by the moving direction, there exists in-plane and out-of-plane motion microactuators. The plane refers to the fixed layer of the microactuator. The in-plane motion indicates that the movement is in a plane parallel to the substrate and the out-of-plane motion means the movement has an angle with the substrate.

If classified by driving principle, microactuators can be categorized into 4 types, i.e., electrostatic, electrothermal, electromagnetic and piezoelectric actuations [1], [2]. The electrostatic actuation utilizes the electrostatic force between the opposite charges on the surfaces of different electrodes. Electrostatic force is not used in driving macroscopic machinery due to the small force (in the magnitude of $\mu \mathrm{N}$ ). Instead, it is widely used to drive micro devices due to the large surface-area-to-volume ratio, the small mass and short distance between electrodes in the micro electrostatic actuator, which provides enough force to drive the micro structure. The electrothermal microactuator [3]-[7] utilizes thermal expansion and contraction of the structures. The heat source is from the resistive heating generated by the electric current. Cooling can be achieved by conduction, convection and radiation dissipations. Usually electrothermal actuation can generate larger forces and displacement than electrostatic actuators, but the power consumption is much higher. Electromagnetic microactuator [8]-[12] uses the magnetic force exerted on the magnetic material or the Lorentz force exerted on the conductors. It consumes high power and requires external magnetic field. Piezoelectric microactuators [13]-[17] uses the inverse piezoelectric effect, i.e., piezoelectric materials generate deformation when subject to electric field. Thus far the fabrication of piezoelectric materials is not highly compatible with semiconductor process. All of the four conventional driving principles have been used to make microactuators to generate out-of-plane motion.

Among the four driving principles, the electrostatic actuation has advantages of low power consumption, high efficiency, high compatibility with semiconductor process, simple and diverse 
structures. As a result, a lot of electrostatic microactuators have been developed for optical switches [18]-[20], optical attenuators [21], [22], tunable optical filter [23] and scanning displays [24]-[27]. All of those applications require out-of-plane motions, which highly desire large rotation angles in order to increase the switching number of ports, the attenuating range and tunability as well as enlarge the display area for scanning display application. There are two types of conventional out-of-plane electrostatic actuators, i.e., parallel-plate (or gap-closing) and vertical comb-drive actuators, both of which utilize the attractive force to pull the moving electrode toward the fixed electrode.

\subsubsection{Electrostatic attractive-force out-of-plane rotation actuators}

A parallel-plate actuator can be considered as a capacitor with one plate fixed and the other movable. Its schematic is shown in Fig. 1.1. By applying electric potentials between the two plates, the movable plate subject to the electrostatic attractive force moves towards the fixed plate. With the movement of the movable plate, the gap between the plates, i.e., $g$, is closed. In this case, it is also called gap-closing actuator. The movable plate becomes stabilized when the attractive force is balanced by the mechanical restoring force of the spring.

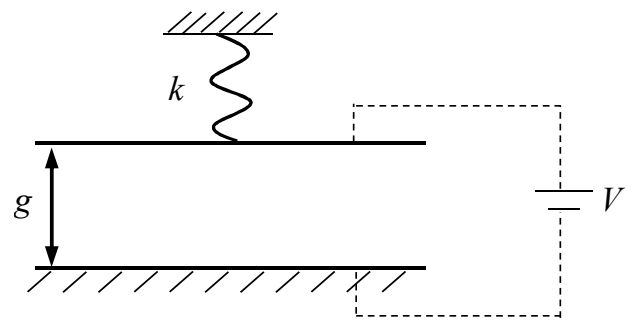

Fig. 1.1 Schematic of parallel capacitor.

Although the parallel-plate actuator is easy to be implemented due to its simple structure, the displacement is limited by the initial gap between fixed and movable electrodes. Furthermore, the parallel-plate actuator suffers from the "pull-in" instability which limits its displacement to less than $1 / 3$ of the initial gap [13]-[15] and causes low operational stability. The "pull-in" is caused due to the fact that electrostatic force is inversely proportional to the square of the gap. As the gap reduces, there is a point beyond which the upward mechanical restoring force cannot balance the downward electrostatic force anymore and consequently the movable plate is pulled towards the fixed plate until they contact. 
Much work has been done to increase the travel range of the parallel-plate actuator. One method is to enlarge the initial gap. Self-assembly is used for surface micromachined parallel-plate actuator to achieve this [19]. The micromirror in [19] was actuated by the electrode plate under it. The micromirror together with its supporting frame is lifted by serval self-lifting assembly arms to increase the gap between the micromirror plate and its underneath electrodes. The micromirror is able to achieve $6.6^{\circ}$ rotation angle under $180 \mathrm{~V} \mathrm{DC}$. However, the increase of the initial gap will lead to large driving voltage, and the self-assembly structures require large area. Another method to improve the displacement of the parallel-plate actuator is to use leverage structure to amplify the displacement [31]-[34]. The parallel-plate actuator and the actuation beam are located at two sides of the levering beam as shown in Fig. 1.2 and Fig. 1.3. However, a long leverage beam is needed if a large displacement is to be achieved, which requires a lot of space. In addition, the actuator still suffers from the "pull-in" instability, which lowers the operational stability.
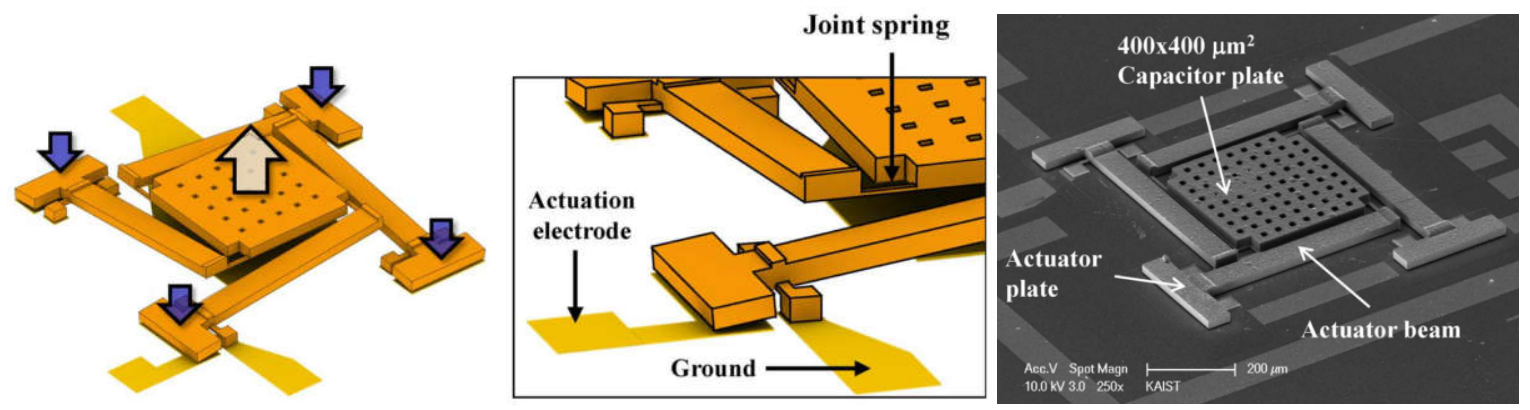

Fig. 1.2 Schematic view of the MEMS variable capacitor and SEM photographs of the fabricated device [32].
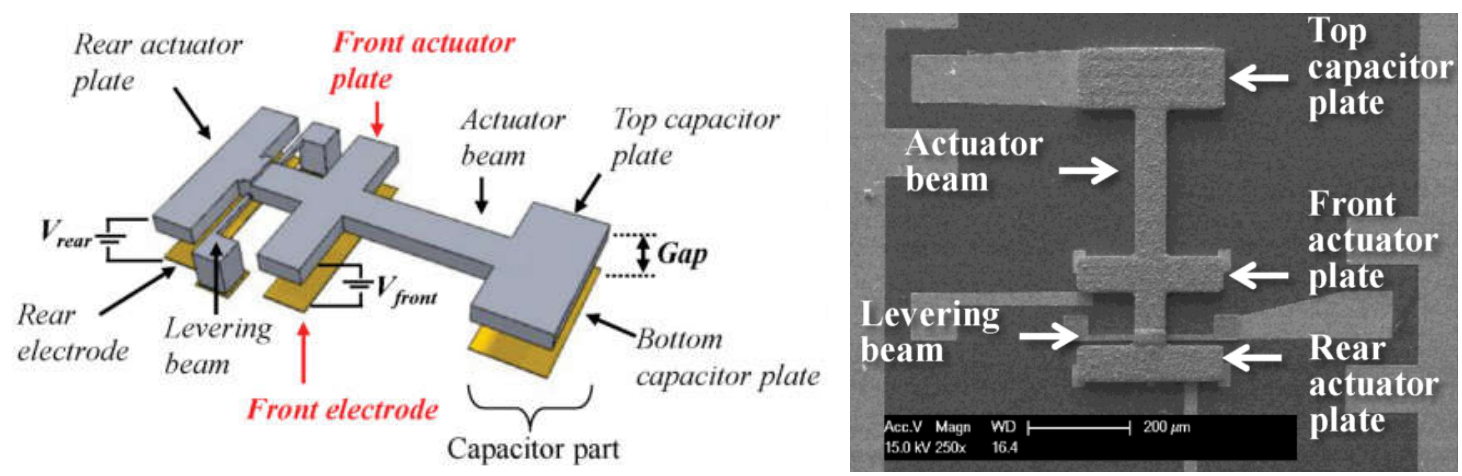

Fig. 1.3 A voltage controlled C-V response tuning and SEM image of the fabricated device [34].

One method to avoid the "pull-in" effect existed in a parallel plate electrostatic microactuator is to design the electrodes in an offset-drive configuration. Instead of putting an electrode right beneath the movable plate, two electrodes are located on both sides of the movable plate [35]-[38]. 
Consequently, the "pull-in" effect is avoided and the movable plate is mainly driven by the electric fields between it and the side electrodes. The offset-drive configuration has similar structure to vertical comb-drive which is usually fabricated using bulk micromachining process [36], [39]. However the offset-drive method is suitable for surface micromachining process which can significantly extend the travel range of out-of-plane actuators. Rosa et al. [35] presented an offsetdrive structure with two tapered shaped external electrodes located on each side of a buckled cantilever beam as shown in Fig. 1.4. Prototypes were fabricated and it was proved experimentally that the cantilever beam was able to travel almost the entire movement range. Large actuation voltage is required (more than $160 \mathrm{~V}$ ) to achieve the full range travel and the actuator requires a pre-lifting movable plate using the residual stress gradient which is hard to be controlled. A similar structure was presented in [36]. The substrate effect was analyzed and it was proved that the driving voltage could be lowered by removing the substrate under the moving plate. According to the analysis, the tapered side electrode design in [35] is a variation of the series capacitor mechanism [40]. However, the presented actuator in [36] is a fixed-fixed beam which is difficult to be utilized to drive some other relative large microstructures.

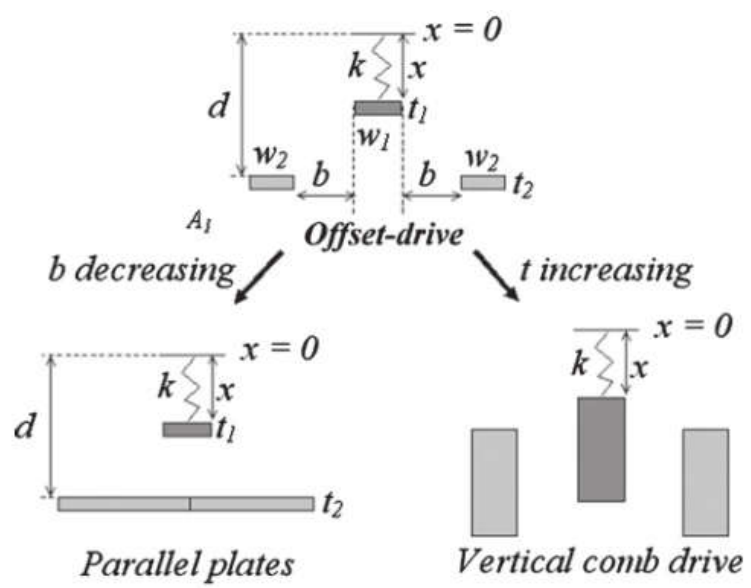

Fig. 1.4 Basic structures of offset-drive, parallel plates and comb-drive [35].

Nonlinear springs are also developed to cancel the nonlinearity relationship between the electrostatic attractive force and the gap between electrodes in a parallel-plate microactuator [41][43]. A method of designing the nonlinear spring was presented in [42] and a macro-scale test was performed to verify the design model. The proposed design methodology can be potentially used in micro-scale for microactuators. However, due to the limitation of the fabrication process, the 
nonlinear spring is difficult to be fabricated in a micromachined out-of-plane actuators. As a result, the proposed methodology was only verified using an in-plane motion microactuator [43].

In addition, control methods are also used to extend the travel range of the parallel-plate actuator such as charge control [40], [44]-[47] and voltage control [48], [49]. However, the control methods either increase the driving voltage or require a sensing mechanism which adds complexity to the design. Moreover, even though the parallel-plate actuator can be controlled to travel the entire range of the initial gap, the displacement is still limited by the initial gap between the plates. The above limitations determine that the parallel-plate actuator is not suitable for outputting a large out-of-plane displacement.

The other type of electrostatic attractive-force actuator, i.e., vertical comb-drive actuator, is more suitable for applications which require large out-of-plane displacements, especially in driving scanning micromirrors [50]-[61]. The vertical comb-drive consists of an array of stationary fingers interdigitated with another array of movable fingers with vertical offset. When a voltage is applied between them, the movable comb will move towards the fixed comb. The electrostatic attractive force generated between the two arrays of combs is independent of the displacement [62]. However vertical comb-drive actuators suffer from the side "pull-in" instability if the fingers are not properly aligned in the transverse direction due to the imperfection in fabrication or external vibration [39].

In order to avoid the side "pull-in" instability and make an initial vertical offset between the fixed and movable combs, many fabrication processes have been developed. The surface micromachining is used to fabricate a staggered vertical comb-drive based on (SUMMiT-V) [50] as shown in Fig. 1.5. The actuator was used to drive a mirror plate $(137 \mu \mathrm{m} \times 120 \mu \mathrm{m})$. The static mechanical rotation angle was measured to be $\pm 5.9^{\circ}$ under a $6 \mathrm{~V}$ DC driving voltage. One drawback is that the travel range of the mirror plate is limited by its size and consequently this design is suitable for small mirrors.

Another comb-drive actuator which requires only one structure layer has been recently presented in [63], [64]. The vertical offset between the movable comb and fixed comb is obtained by the residual stress along the bending beam. Experimental results showed that a vertical displacement of $4.81 \mu \mathrm{m}$ can be reached at $150 \mathrm{~V}$. Side "pull-in" instability happens when the applied voltage is above $160 \mathrm{~V}$. The achieved travel range is relatively small, which is only about 
$22 \%$ of the entire travel range. The residual stress gradient, which is difficult to be accurately controlled, leads to the small initial vertical offset between fixed and movable combs and then a small displacement
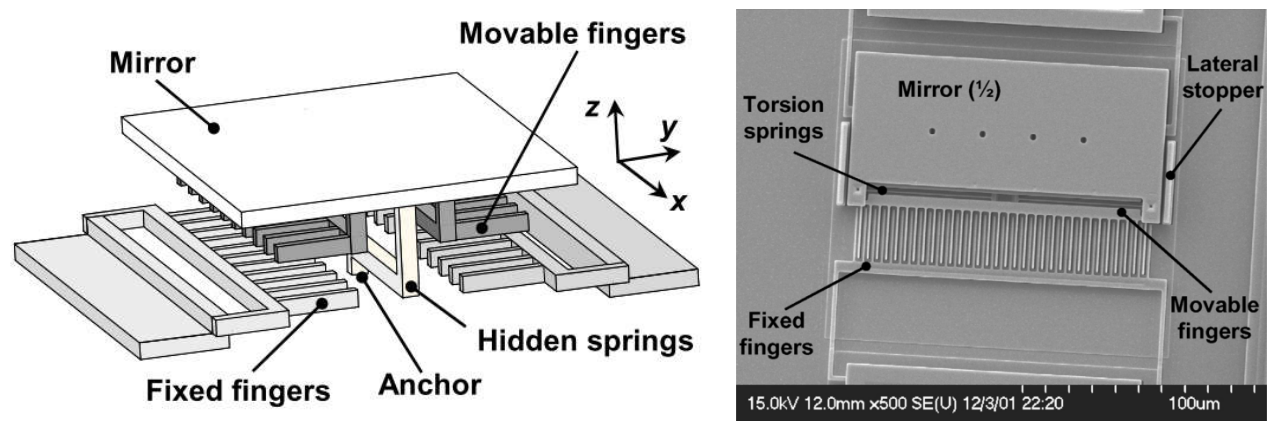

Fig. 1.5 Left: The schematic structure of the proposed micromirror. Right: The SEM micrograph of the fabricated micromirror with the other half of the micromirror removed [50].

Bulk micromachining is more popular in fabricating the vertical comb-drives which is able to provide large travel range to the actuator and the driven microstructures [39]. One challenge of the bulk micromachining is the alignment of the fingers. Many processes have been developed with different methods to help solve this issue. For example, a fabrication process based on SOI wafer bonding was developed to avoid the misalignment of the combs in [52]. Measurements show that less than $0.1 \%$ misalignment can be obtained using the presented fabrication process, which enables the perfectly aligned device to travel $98 \%$ of the full range. Other fabrication processes with their own alignment mechanisms are also presented, such as displaced anchor process [65][67], single wafer process [68]-[70], polysilicon trench re-fill process [71]-[73], custom SOI wafer process [52], [74]-[77], SOI wafer process [53], [78]-[85], single device layer process [86]-[91] and multiple wafer process[92]-[94].

The displacement of the vertical comb-drive is limited by the smaller thickness between the movable comb and fixed comb [39], [95]. The comb thickness of the bulk micromachined actuators is usually in tens of microns which limits their DC travel range. As a result, many vertical comb-drives need to operate under resonant frequency to obtain large deflections [52]. For example the comb-drive actuated micromirror presented in [52] is able to achieve only $4^{\circ}$ optical DC scanning angle. However, under the resonant driving mode, the optical scanning angle increases to about $40^{\circ}$. 
Besides driving the actuator in resonant mode, many other studies have been conducted to extend the travel range of vertical comb-drive actuators. For example, a dual mode comb-drive [93], [96] is able to enlarge the actuation force and the travel range. Different from the conventional comb-drive which has one row of fixed comb, the dual comb has two rows of fixed combs located below and above the movable comb as shown in Fig. 1.6 (b). A large DC optical scanning angle, i.e., $32^{\circ}$ was measured for the $1 \mathrm{D}$ scanning micromirror driven by the dual mode comb-drives at 100 V DC [93]. However, the dual mode configuration requires 3 structure layers with relative large gap between each other, which leads to complicated fabrication process. Furthermore, due to the process limitation, the lower fixed comb and the upper fixed comb are usually asymmetrical with respect to the movable comb in the middle, which result in complicated driving approach.

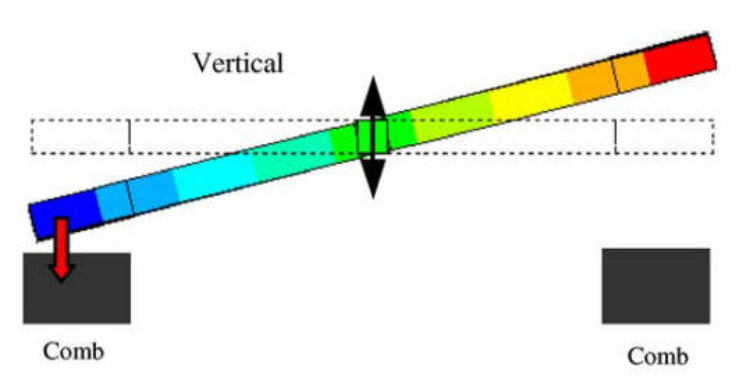

(a)

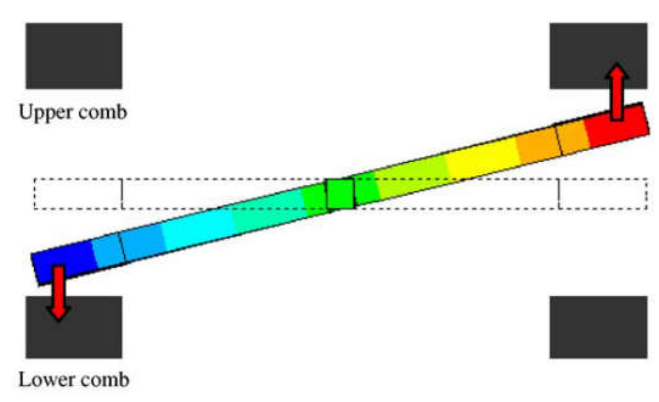

(b)

Fig. 1.6 (a) Comb-drive with fixed combs in one side of the movable comb (b) Dual mode combdrive [93].

Another way to improve the deflection of vertical comb-drive is to use angular vertical combdrive (AVC). According to the types of the offset the vertical comb-drives can be categorized into staggered vertical comb-drive (SVC) and angular vertical comb-drive (AVC). The movable combs of SVC have a vertical translational initial offset with the fixed comb, and the movable combs of AVC have an angular initial offset with the fixed comb. It has been proved that AVC with relatively thin fingers has a $60 \%$ larger deflection than that of the SVC of the same dimension [57].

From the literature review in this section, it can be concluded that the parallel-plate actuator has the advantage of simple structure. However, the "pull-in" effect and the small gap between the two electrodes limit the out-of-plane deflection. In contrast, the vertical comb-drive can generate larger displacement due to large vertical dimensions and has no direct "pull-in" effect. Nevertheless, more complicated fabrication processes are required to generate the initial vertical 
offset between the fixed and movable combs and to avoid the finger misalignment. Furthermore, it suffers from the side "pull-in" instability which even though can be avoided by introducing proper alignment mechanism in the fabrication process, will still be a potential unstable factor when exposed to relative large external vibrations. A novel type of electrostatic repulsive-force actuators has been previously developed which can generate repulsive electrostatic force to push the movable structures out-of-plane. It can overcome some of the disadvantages of parallel-plate and vertical comb-drive.

\subsubsection{Electrostatic repulsive-force out-of-plane actuators}

Different from the attractive-force electrostatic actuators which utilize the attraction force to bring two electrodes together, repulsive-force (or repulsive-torque) actuators use the electrostatic net force to push the movable electrodes away from the underlying electrodes whose effect is repulsive [97]. It is noted that the "repulsive-force" or "repulsive-torque" here is not simply generated from repelling force between charges of the same polarity. They are the net force of a number of electrostatic forces which are exerted on the top and bottom surfaces of the movable electrode. The total force exerted on the top surface is upward, and the total force exerted on the bottom surface is downward. Due to the asymmetrical electric filed around the movable plate, the force on the top surface is larger than that on the bottom surface. As a result, the resultant electrostatic force pushes the movable structure away from its counterpart. The asymmetrical electric filed is generated by a series of properly configured electrodes. It has the advantage over the attractive-force actuators of larger out-of-plane displacement and higher reliability since 1) Its displacement is not limited by the space between the movable and fixed electrodes 2) It does not suffer from the "pull-in" instability that encountered by parallel-plates and comb-drives [1], [2], [28], [29], [98].

Different structures of repulsive-force actuator have been presented. A 3-layer electrostatic actuator, which can generate repulsive force, is reported in [99], [100] as shown in Fig. 1.7. The 3 electrodes constructed by 3 different layers are all vertical aligned with an isolation layer (oxide) sandwiched between each two layers. The top electrode is the movable structure while the bottom 2 electrodes are fixed. By applying the same electric potential to the top two electrodes, and a different potential to the most bottom electrode, an out-of-plane electrostatic force is generated on the movable electrode and pushes it to move upward. The advantage of the design is that it has a 
large fill-factor similar to that of the parallel-plate actuators. However, due to the fabrication limit, the tip of the movable structure suffers from a stiction force ( $F_{c}$ in Fig. 1.7$)$ which prevents the tip from separating with the underlying fixed metal layer. This significantly limits the actuator's displacement. It is reported that the driving voltage is limited to a low value, i.e., $40 \mathrm{~V}$, beyond which it will cause severe current leakage of the sandwiched isolation layer. A small out-of-plane displacement is obtained which is less than $0.8 \mu \mathrm{m}$.

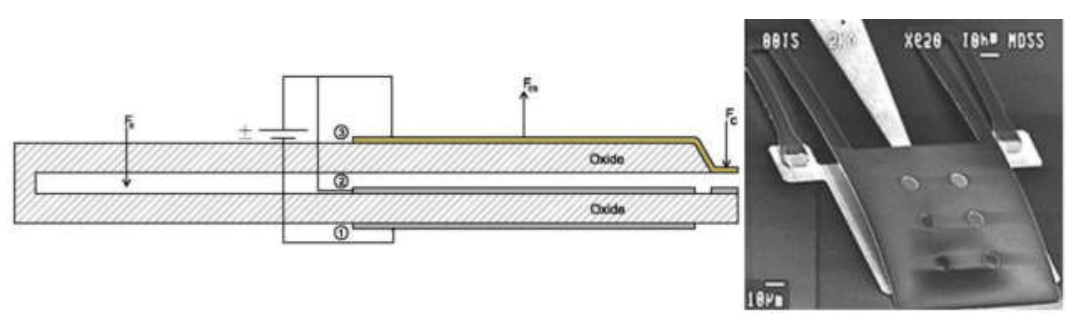

Fig. 1.7 Left: Schematic of 3-layer repulsive-force actuator. Right: Scanning electron microscope image (SEM) of a fabricated device with $100 \times 100 \mu \mathrm{m}$ paddle [99].

Another 2-layer repulsive-force out-of-plane actuator has been presented in [1], [2], [28], [29], [98] which requires only two thin layers. The first design of this type of actuator was presented in [101] which was used to drive a micromirror plate to realize the out-of-plane translation. The configuration of the electrodes is shown in Fig. 1.8. The top layer is the movable structure, and the bottom layer is the fixed electrodes. The electrodes are finger shaped and are called fingers hereafter. There is a corresponding fixed finger under each moving finger, which are both subject to the same electric potential. There are unaligned fixed fingers located on the sides of each pair of movable fingers and its underling fixed fingers. The unaligned fingers are applied different electric potentials. There are two fixed electrodes between the two neighboring moving electrodes as shown in Fig. 1.8 (b). The electrostatic force is exerted on both the top and bottom surfaces of the movable fingers with the net force upward due to the electrode configuration. As a result, the moving fingers are driven to move away from their underlying electrodes, and the effect of the force is repulsive. The actuator was fabricated using a 2-layer PolyMUMPs process and is able to achieve a vertical stroke of $1.75 \mathrm{um}$ at $50 \mathrm{~V} \mathrm{DC}$. The displacement is more than 3 times of that of the conventional MUMPs translational micromirrors. A similar actuator was also presented as Actuator $B$ in [102] and a repulsive-force actuator with different electrode configuration was presented as Actuator A. However, Actuator $A$ is difficult to be fabricated since it requires an 
insolation layer to be placed between the driven microstructure and the movable fingers. The applications of using the actuators in adaptive optics were presented in [102], [103].

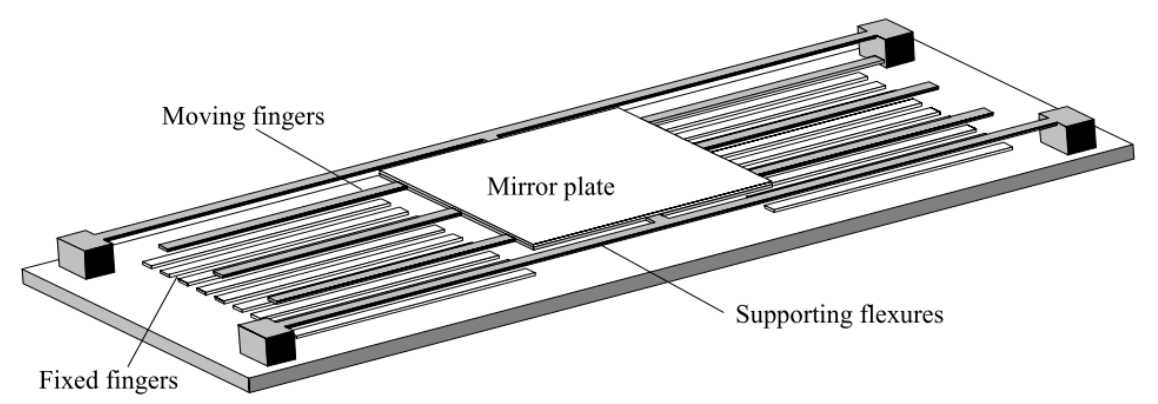

(a) Structure of the micromirror

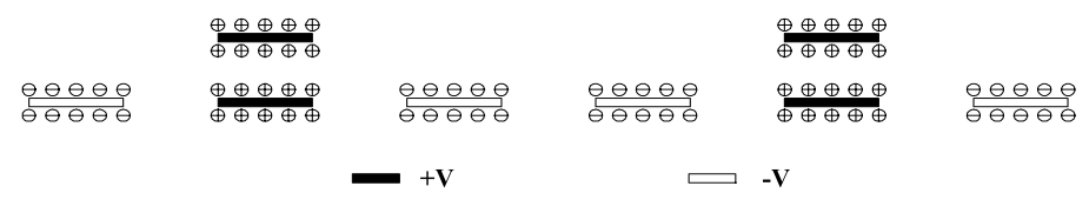

(b) Section view of fingers

Fig. 1.8 The novel translation micromirror for adaptive optics [101].

In addition to the translation actuator, the repulsive force driving mechanism was also used to construct out-of-plane rotation actuators [97], [104], [105]. The vertical translation is converted to out-of-plane rotation by anchoring one side of the movable fingers. A rotation actuator was presented in [97] and was used to form a 1D scanner. A detailed electric filed analysis has been given and an analytical model has been built which matches well with the simulation and experimental results. The proposed actuator was fabricated using a 2-layer MUMPs process. The testing results showed that the micromirror has a rotation angle of $0^{\circ} \sim 2.1^{\circ}$ with $0 \sim 200 \mathrm{~V} \mathrm{DC}$ driving voltages. The frequency response of the micromirror was tested to be $150 \mathrm{~Hz}$. A design optimization model for the actuator was built in [105] using a hybrid approach by combining numerical 2D electric field simulation and analytical derivations. The hybrid approach provides a good guidance to create the math model for other electrostatic actuators. By using the optimization model, the finger width can be optimized by knowing some basic parameters of the actuator such as the actuator's width and length, the spring stiffness and the desired maximum driving voltage. The actuator having the same size and spring stiffness as the one presented in [97] was optimized using the proposed optimization model and fabricated (SEM image shown in Fig. 1.9). The test result shows that a rotation angle of $4.4^{\circ}$ can be achieved at $200 \mathrm{~V}$ DC and there is more than $100 \%$ 
improvement compared to the non-optimized actuator presented in [97]. The application of using the rotary actuator in automated microassembly was presented in [106].

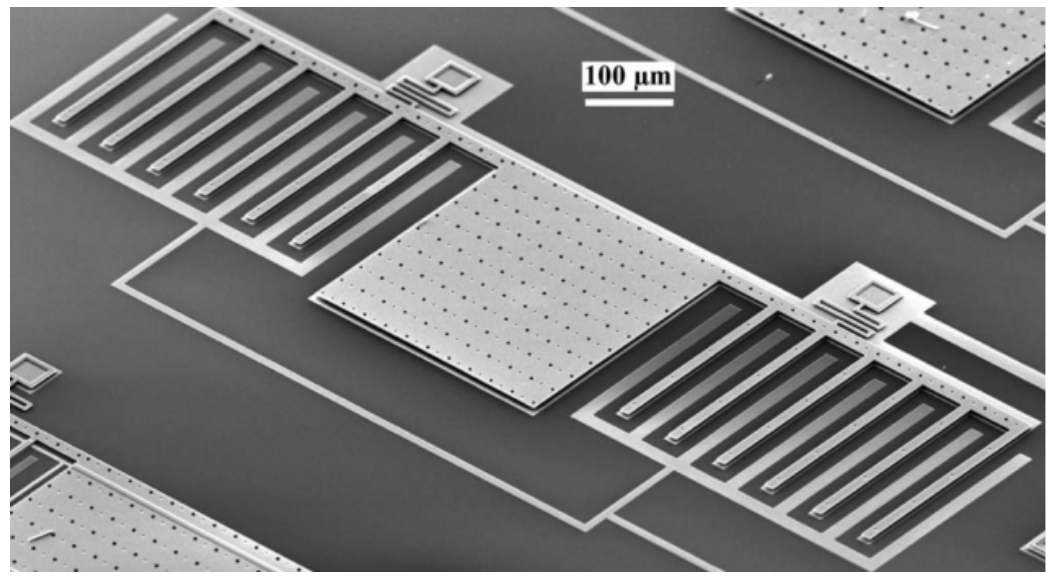

Fig. 1.9 SEM image of the optimized actuator fabricated using the PolyMUMPs process [105].
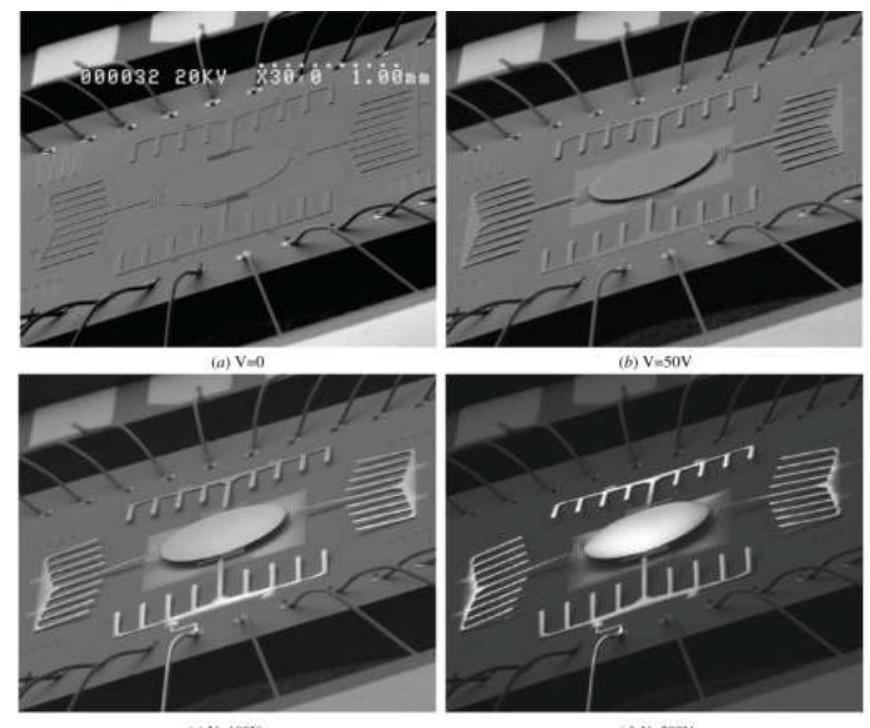

(c) $V=100 \mathrm{~N}$

(d) $\mathrm{V}=200 \mathrm{~V}$

Fig. 1.10 SEM images of the actuator subject to different voltages [107].

One important application of the repulsive-force actuators presented in [97], [105] is to drive a 2D scanning micromirror [107] which is shown in Fig. 1.10. Four identical actuators are used to drive a center micromirror plate. The measured results show that the micromirror has a static outof-plane translation of $86 \mu \mathrm{m}$ at $200 \mathrm{~V}$ DC. The micromirror can also operate in rotation mode which can achieve $\pm 1.5^{\circ}$ rotation angles under $200 \mathrm{~V}$ maximum driving voltage. Some applications of the micromirror are developed such as laser scanning vector display [108] and automotive head-up display [109]. 
Compared to the attractive-force actuators the presented repulsive-force actuators [97], [105], [107] is superior since it suffers from neither the direct "pull-in" effect nor the side "pull-in" instability. Furthermore, the travel range is not limited by the initial gap between the two opposite electrodes. They can be used for both translation [102] and rotation [97], [105], [107] driving. It also has a simple structure which requires two thin layers and can be fabricated using low cost commercialized surface micromachining process instead of some customized processes which are high cost. However, the currently developed repulsive-force actuators have three major disadvantages. The first is the rough surface quality due to releasing holes and dimples that are introduced in the surface micromachining process [110]. It limits the micromirror to be used in some optical applications which have high requirement on the micromirrors' surface quality. One potential approach to solve this is to develop a bonding technique to bond a high surface quality silicon mirror plate on top of the surface micromachined mirror plate. One promising methodology has been presented in [111]. The second issue is the relative low fill-factor of the device, which is similar to the vertical comb-drive actuators. This issue is attributed to the configuration of the electrodes that requires unaligned fixed fingers on both sides of the movable finger. One possible solution to this issue has been presented in [102] as Actuator $A$ which introduced an insolation layer between the movable fingers and the driven microstructure. The mechanism is difficult to be implemented due to the limitation of the fabrication process. Another solution has been reported in [103], which utilizes the movable finger as both the actuation beam and the spring. However, the presented solution is only limited to the actuators with small out-of-plane deflection. A feasible solution to improve the fill-factor of repulsive-force actuators with large vertical deflections has not been proposed yet. The third issue is the relative small force and then small out-of-plane deflection generated by the actuator. This limitation is critical and needs to be addressed in order to use the repulsive-force actuator in practical applications because: 1) A voltage as high as about $200 \mathrm{~V}$ [107] has to be applied to the existing repulsive-force actuators in order to generate enough force/torque, which is close to the breakdown voltage of the substrate insulation layer. For example silicon nitride $(0.6 \mu \mathrm{m})$ layer on the substrate acts as the insulation layer in PolyMUMPs [112]; and 2) If voltage lower than $150 \mathrm{~V}$ is to be used, the size of the actuator needs to be significantly increased in order to provide enough force/torque. For example, the size of the micromirror developed in [107] has a size of $3.3 \times 3.3 \mathrm{~mm}$. Further increase the size of the micromirror may lead to a bulky device and deteriorate the dynamic performance of the micromirror due to the 
increased damping. Thus, the development of a repulsive-force rotation actuator with output torque (and then rotation angle) much larger than that of the existing ones is the first objective of this thesis. In addition, the microactuator that is to be developed should have low cost and high fabrication yield, which is suitable for mass production and various applications.

\subsection{Micromirror based laser scanning display review}
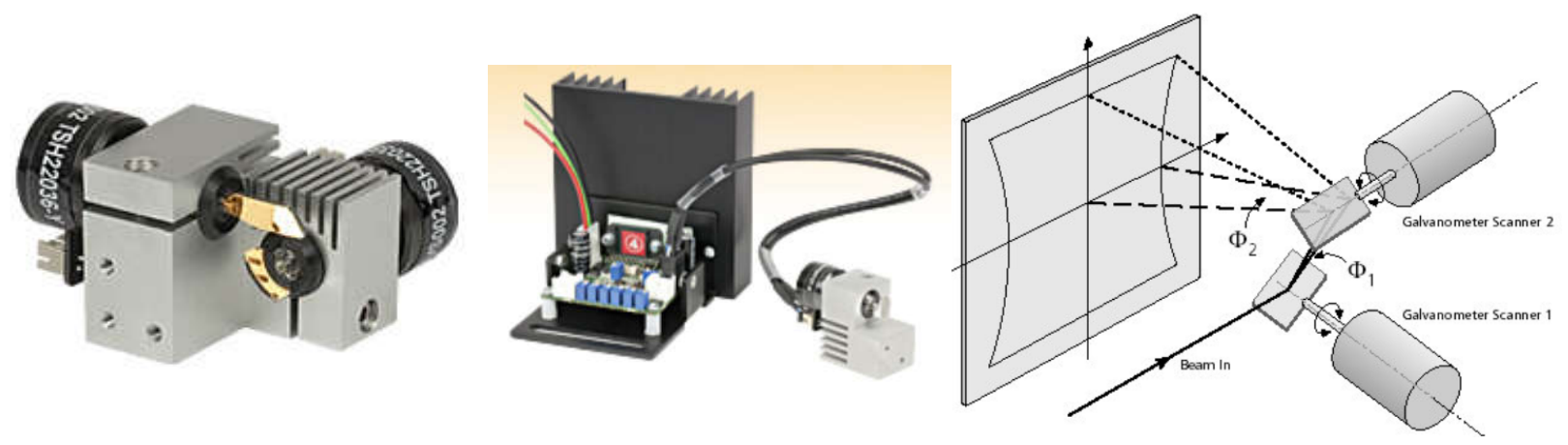

Fig. 1.11 Left: Galvanometer scanner and its driving circuit [113] Right: 2 axis galvanometer scanning schematic [114].

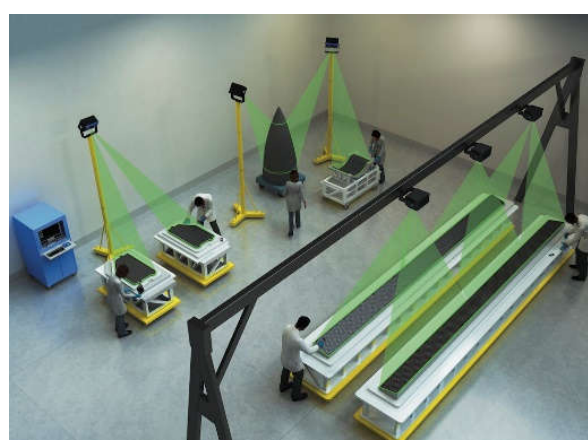

(a)

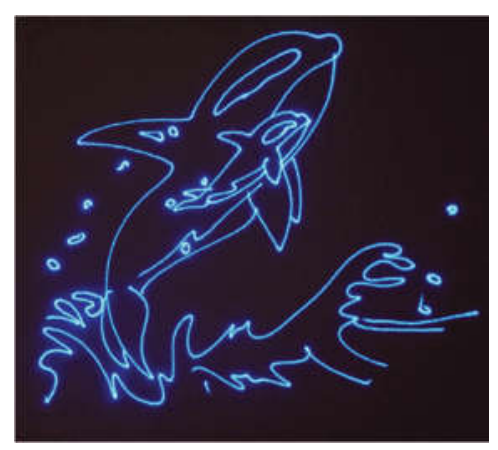

(b)

Fig. 1.12 (a) Laser projection based assembly and tooling guidance [115] (b) Laser show [116].

The laser scanning display (LSD) is widely used in manufacturing such as assembly guidance [115] and laser shows [116]. Conventionally, the galvanometer scanner shown in Fig. 1.11 is used to steer the laser beam to scan through desired trajectories. It has large mechanical rotation angles $\left(> \pm 10^{\circ}\right)$ with frequency bandwidth higher than $1 \mathrm{KHz}$ [115]. However, such scanning system is composed of two large scanners and some bulky peripherals such as servo circuits, driving module and heat sinks. The scanning micromirrors based on out-of-plane electrostatic actuators have advantages of small volume, low power consumption and simple optical setup [27], [93] and hence are getting more attentions. 
There are two schemes of micromirror based laser scanning display, i.e., raster and vector scanning display. In the raster scanning display, the laser beam is controlled to scan through a display area line by line. As a result, one scanning axis is required to have a faster scanning frequency than the other to perform raster scanning. In the vector display, the laser beam only scans through lines and curves of the desired vector patterns. According to different scanning schemes, the micromirror types are also different. The raster scanning display requires resonant scanning micromirror with one or both axes working under their resonant frequency so that a relative high scanning frequency and large scanning angle can be achieved. The two axes of this type of micromirror is divided into slow axis and fast axis. The fast axis works under its resonant frequency so that the excited resonant vibration of the actuator is able to steer the micromirror to scan through a large area rapidly. The slow axis has relaxed restriction and may work at resonant or non-resonant mode. The vector scanning display requires quasistatic micromirror which can be stabilized at any rotation angle in the operation range and is able to steer the laser beam to track a desired trajectory point by point to generate a vector graphic. It is noted here that some micromirrors are able to be used for both raster scanning and vector scanning. However, they are always more suitable for one of the scanning scheme and cannot achieve good performances for both.

An advantage of the resonant micromirror over the quasistatic one is the large scanning angle due to the large displacement under the resonant mode. One example of the resonant micromirror was presented in [93] as the eye-type one-axis scanner. It has an optical scanning angle of $32^{\circ}$ when driven by sinusoidal voltage under the micromirror's resonant frequency in the range of 22.1 $\sim 24.5 \mathrm{kHz}$ with $100 \mathrm{~V}$ DC bias voltage. It is used together with a huge galvanometer mirror to display an image with extended graphics array $(\mathrm{XGA})$ resolution $(1024 \times 768)$ as shown in Fig. 1.13 (a), which will significantly increase the size of the display module. A biaxial resonant micromirror driven by vertical comb-drive was developed [26]. The micromirror was fabricated using a dual layer polysilicon SOI process and was packaged in a vacuum package to improve the scanning angle. Relative low driving voltage $(60 \mathrm{~V})$ is required for the fast axis to achieve large optical scanning angle, i.e., $60^{\circ}$. The low driving voltage has advantages of high reliability and compatibility with some consumer electronics. A $512 \times 1024$ pixel image is generated using the micromirror, as shown in Fig. 1.13 (b). 


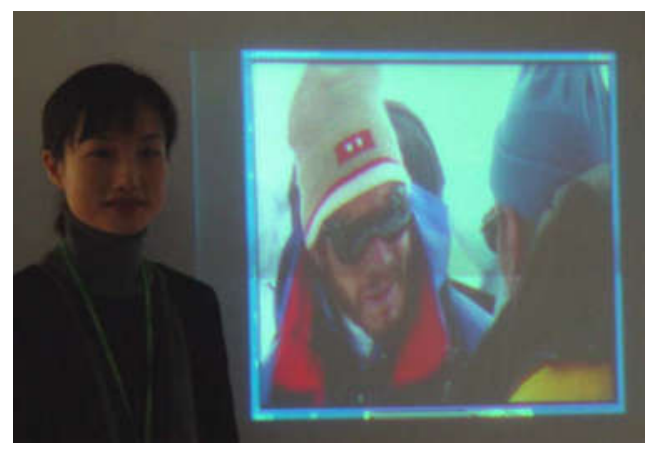

(a)

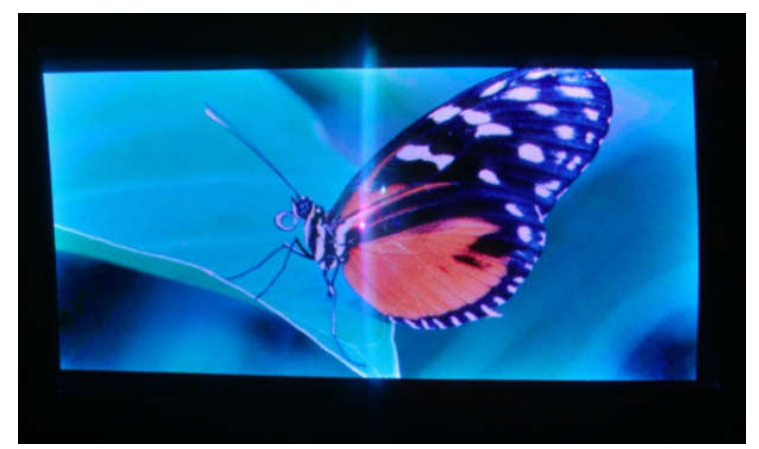

(b)

Fig. 1.13 Display results of laser raster scanning display based on electrostatic micromirrors [26], [93].
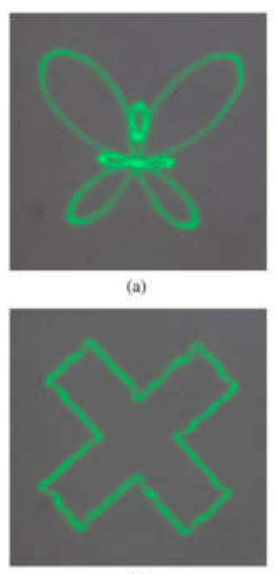

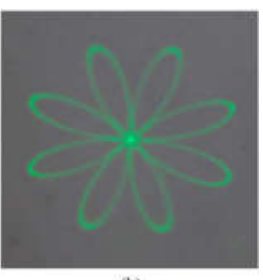

(b)

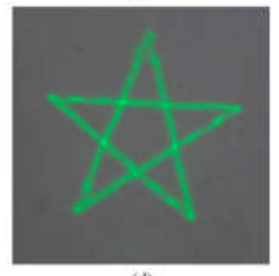

Fig. 1.14 Vector scanning graphics using 2-layer surface micromachined micromirror [108].

Quasistatic micromirrors are also widely studied. A vertical comb-drive driven micromirror having an optical scanning angle of $16^{\circ}$ for both axis was developed in [25]. A micromirror based on electrostatic repulsive-force actuator [107] was previously used to display vector graphics, as shown in Fig. 1.14 [108]. The measurements showed that the micromirror has an optical scanning angle of $\pm 2.5^{\circ}$ for both axes with the aid of a magnification lens. The maximum rotation angle is achieved at $200 \mathrm{~V}$ DC which approaches the breakdown voltage of the insolation layer and may cause a failure of the device. Usually by introducing magnification lens, the small rotation angles widely suffered by quasistatic micromirrors can be magnified. For example, the $16^{\circ}$ optical scanning angle in [25] can be amplified to about $120^{\circ}$ using a customized fisheye lens. However, there is an extra cost on the customization. The micromirror and the display results in [25] are shown in Fig. 1.15. 


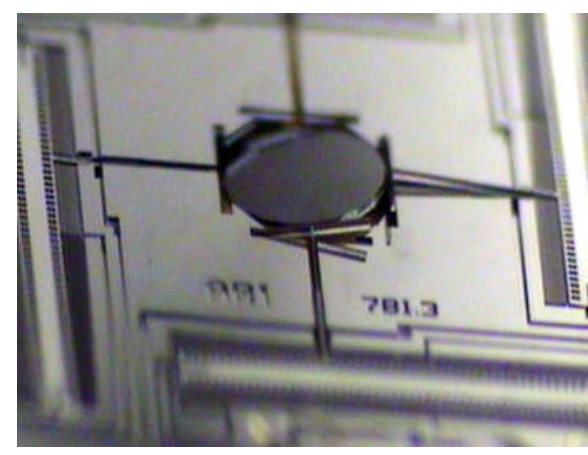

(a)

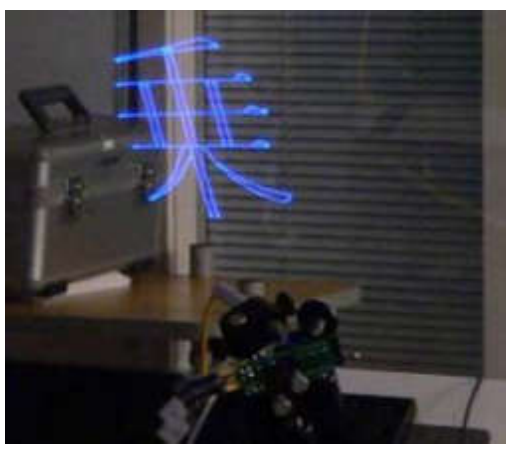

(b)

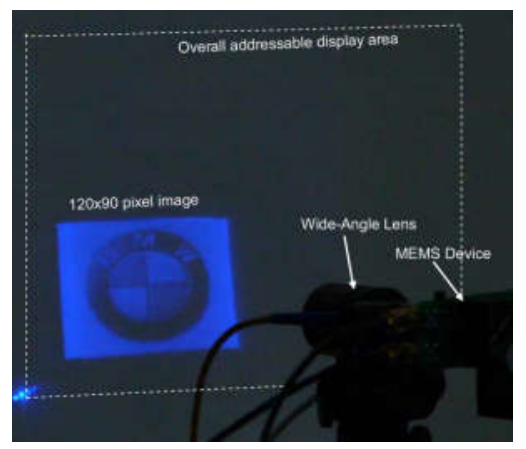

(c)

Fig. 1.15 (a) Micromirror with vertical comb-drive (b) Vector scanning display (c) Raster scanning display [117].

Raster scanning display is capable of generating pixel image which is able to show much more vivid information (as shown in Fig. 1.13) than that of the vector scanning display (as shown in Fig. 1.12(b), Fig. 1.14 and Fig. 1.15 (c)). However, raster scanning display comes with a limitation that the brightness of the display is limited by the maximum laser power that can be used, which is regulated by the laser safety standard [118], [119]. The reason is that in raster scanning display the illuminance of the laser is evenly distributed to all the pixels over the entire display area. In order to increase the average brightness of the display, laser source with much higher output power are required. It has been reported [120] that the laser diodes which are similar to the ones used in optical drives are used for the LSD which could be in the ranges of serval tens to hundreds of miliwatts. According to the laser safety classification specified by IEC 60825-1 standard [121], the laser with this amount of output power will cause eye hazard if directly exposed. If accidentally the micromirror encountered a failure, the laser beam will be concentrated in one spot. The concentrated laser beam may shoot directly or be reflected to the viewer and may cause injury. However, for vector scanning display all of the laser power is concentrated in one single point on the scanned trajectory at one time instant. As a result, usually laser source with lower power can be used [122]. It was reported in [109] that a less than $5 \mathrm{~mW}$ laser was used for a vector scanning display.

For some applications such as the assembly guidance and automotive head up display, only simple patterns are to be displayed. Vector scanning display is fully capable of such applications. Also considering the vector display requires laser with much lower power than that of the raster scanning display, the micromirror based vector scanning display is ideal for such applications. 
Usually low cost and high fabrication yield micromirror is desired which is suitable for mass production and can reduce the overall cost of the display module. As a result, the second objective of the thesis is to develop a low cost micromirror for vector scanning display, which is based on the actuator developed in the first objective.

\subsection{Automotive head-up display (HUD) review}

The micromirror based vector display system can be used in assembly and tooling guidance, laser show (as shown in Fig. 1.12) and automotive HUD [109], [123]. In this thesis the micromirror developed in objective 2 will be used to construct an automotive HUD.

HUD was first developed to help the pilots of fighters to aim at the target. The vector patterns are reflected by the combiners and a virtual image is generated in front of the pilot's sight. Later this technology was used on the civilian air planes to aid the pilots. In 1988, GM introduced this technology to the cars. Nowadays, a lot of car companies have provided HUD as an optional package for their high end cars. It is a trend that HUD becomes a standard module of the cars. The reasons can be concluded as follows. 1) With the development of the sensor technology, more and more driving critical information are provided to the drivers to improve driving safety. 2) The development of the networks is changing the way people communicate. As a result, drivers tend to be exposed to a large amount of driving irrelevant information such as calls, text or voice massages and massage from social media. All those information needs to be displayed properly so that they do not interfere with the driving.

The conventional automotive HUD is mainly based on LCD which dominates the market. The system setup of LCD based HUD is shown in Fig. 1.16. Some issues suffered by LCD limits its application in automotive HUD. Firstly, it has low light efficiency which leads to low brightness of the display. The light source of LCD is provided by backlit LEDs. However, most of the energy are lost in the transmission through the polarizer, color filter and the aperture. As a result, about only $7.7 \%$ of the total illuminance generated by the backlight can be illuminated from the display [124]. Usually, a high brightness capability of the display module is required $\left(>15,000 \mathrm{~cd} / \mathrm{m}^{2}\right)$ so that the display can be observed when there is a strong background illuminance such as driving in the daylight. If the HUD does not have the capability to generate a display with high brightness,

as the background illuminance of the display increases, the displayed image will fuse with the background and can hardly be observed. This is called "washout" of the display. Due to the low 
energy efficiency, the image generated by LCD is not high enough to avoid the washout of the image. This issue cannot be solved by simply increasing the power of the backlight which will cause dramatic temperature increase of the display module [125]. Another issue with the LCD based HUD is the tradeoff between the display size and the volume of the system. Since a virtual image is required to be generated in front of the driver about $2 \mathrm{~m}$ away, the size of the virtual image needs to be large enough for the driver to see all details of the displayed information as illustrated in Fig. 1.16. Usually the image generated by the picture generation unit such as LCD needs to be about twice of the size of the virtual image. Furthermore, the relay optics must be even larger than the image. In this case, a large display will lead to bulky HUD system. As a result, an automotive HUD with low cost, compact size and simple structure is desired.

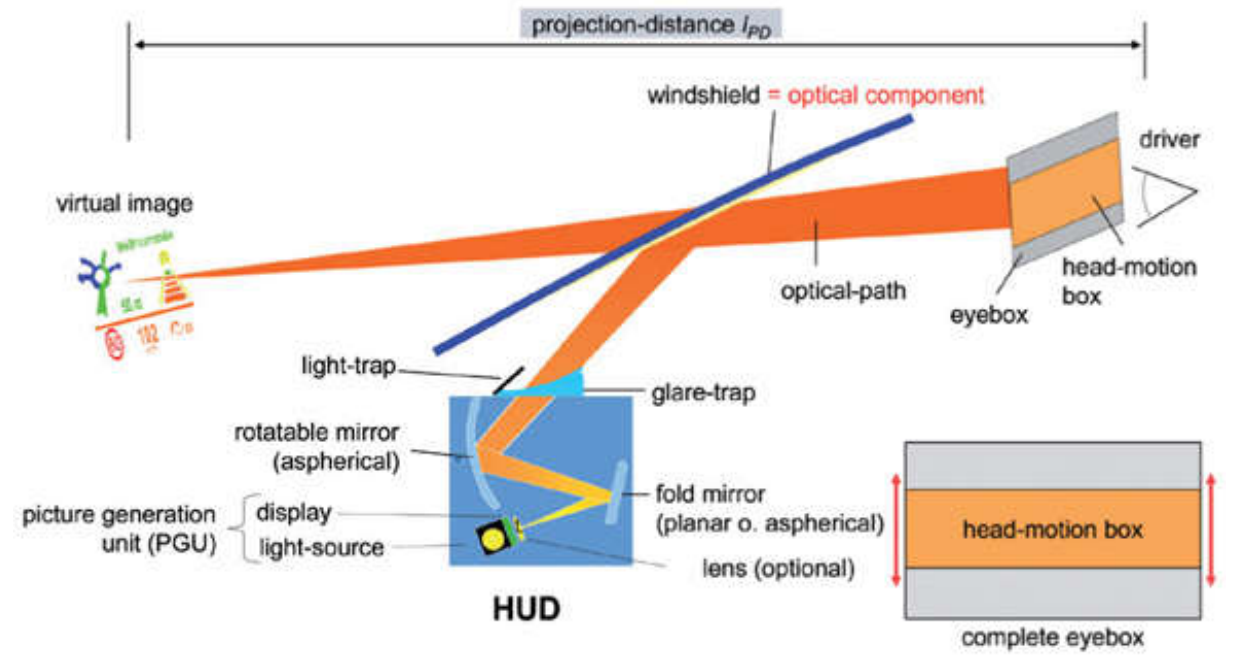

Fig. 1.16 A schematic view of the conventional HUD setup [126].

With the development of the display technologies, digital micromirror device (DMD), liquid crystal on silicon (LCOS), organic light-emitting device (TOLED) and laser scanning light display (LSD) are used to form compact HUD display modules. These technologies are able to overcome the above mentioned limitations of LCD based HUD.

The digital micromirror device (DMD) is a micromirror array which has hundreds of thousands of tiny micromirrors, which is developed by Larry [24]. Each micromirror is actuated by electrostatic attractive force to have two stable states, i.e., $12^{\circ}$ and $-12^{\circ}$ rotation which corresponds to on and off position. The light sources is provided by external LEDs or lasers. Its working principle is illustrated in Fig. 1.17. When the micromirror is at "on" position, the incident light is directed to the projection lens of the system. While at "off" position, the light is directed to light 
absorber which appears to be a dark pixel on the image. A DMD based display module has the advantage of low power consumption, high energy efficiency, fast response time and long working life. A simple prototype of DMD based HUD has been developed in [125]. A commercial DMD based portable projector was used to generate the display image. A 7 inches virtual images lies about $2.5 \mathrm{~m}$ in front of viewer's eye has been generated. Comparison has been made with the LCD based HUD. The experiments show that the overheat issue exists when increasing the output power of the backlight LEDs to generate a brighter image. However, the developed HUD is based on the lab environment, which is a proof of concept. A commercial DMD based augmented reality (AR) HUD [127] has been developed by a German automotive parts company Continential which is a leader in automotive HUD industry. The company has claimed to bring this technology to series production in 2016.

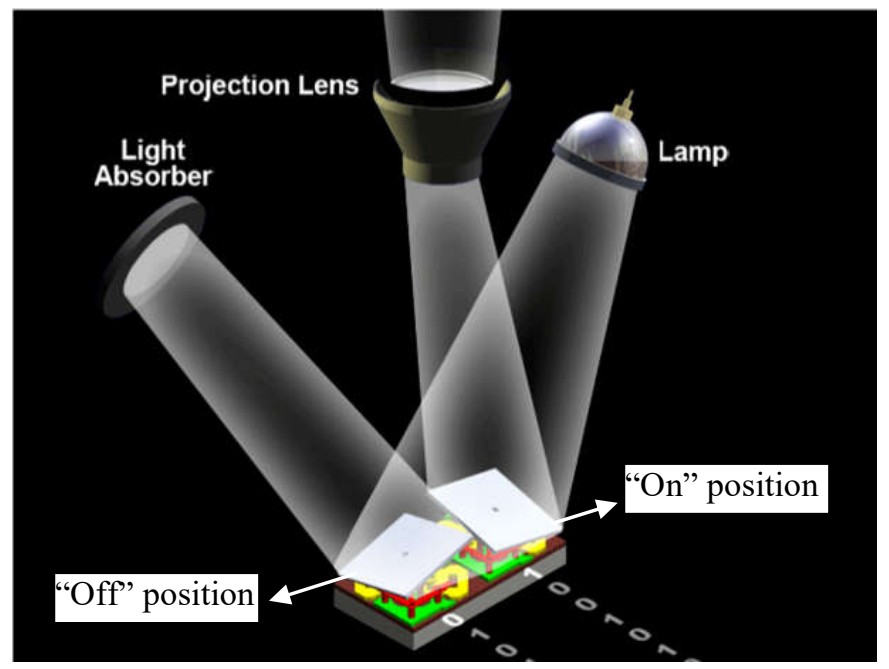

Fig. 1.17 Working principle of DMD based display [128].

Liquid crystal on silicon (LCoS) is an alternative to DMD. Its working principle combines the mechanisms of LCD and DMD. LCoS applies liquid crystal on reflective single crystal substrate. By manipulating the open and close state of the liquid crystal, the incident light to the LCoS is either reflected by the substrate or blocked by the liquid crystal. It has advantages of high resolution, high light efficiency and high contrast ratio. The system cost and volume of LCoS based display module are similar to those of DMD. A phase-only LCoS device has been presented in [124], [129]-[132] and has been used for an automotive holographic HUD system. It has been equipped in Land Rover Evoque as shown in Fig. 1.18. 


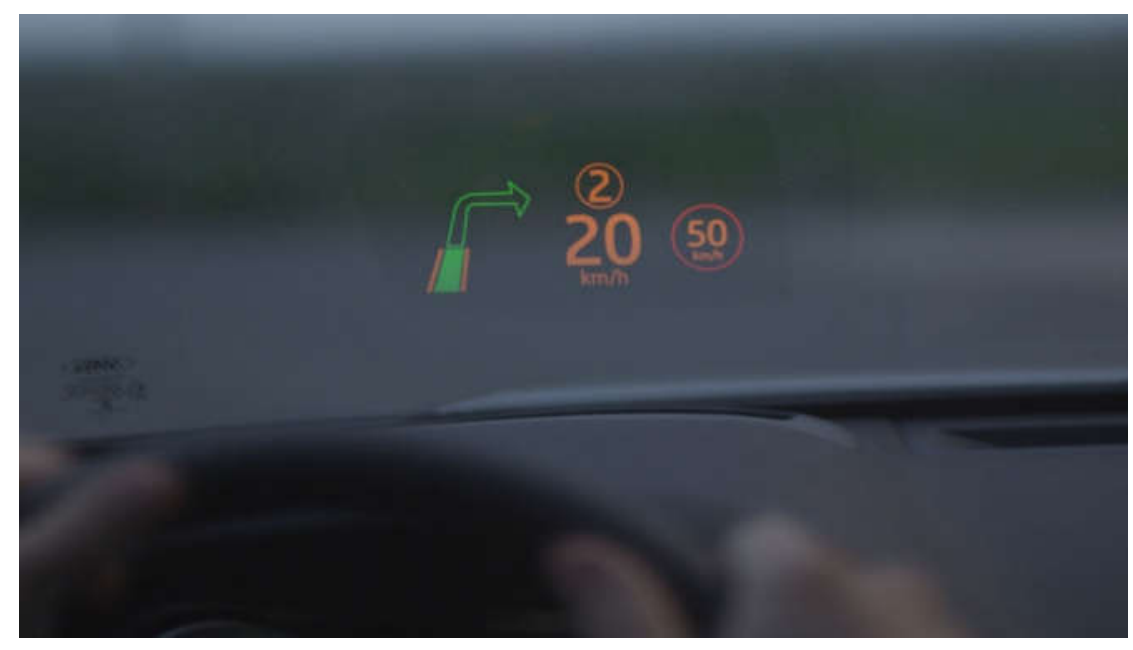

Fig. 1.18 Display result of LCoS based HUD in Land Rover Evoque [133].

Although DMD and LCoS are good at displaying pixel images, they share a common weakness of background glow. Background glow is related to the black-level control ability of the display module. Since the displayed pattern of a HUD is composed of simple symbols, there is a large amount of unused pixels. It is desired that there is no illumination from those pixels, i.e., black pixels. However, DMD and LCoS based display cannot produce true black pixels due to residual illuminance. Consequently the HUD based on them has a glowed background which may interfere with the driving.

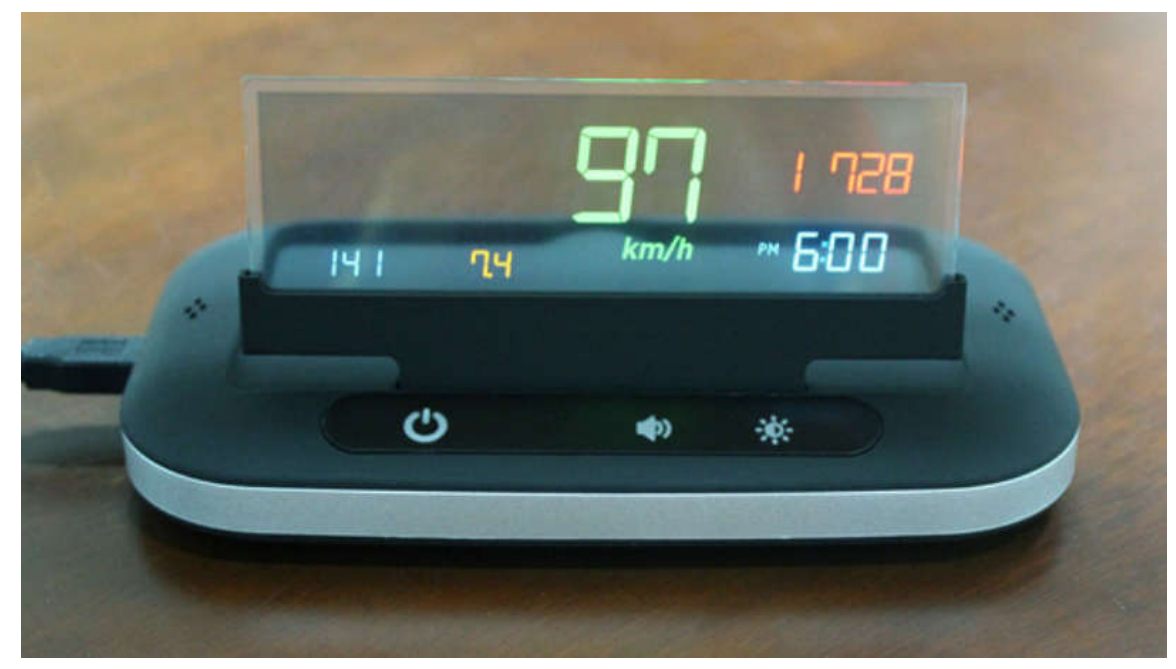

Fig. 1.19 HUD based on TOLED [134].

Transparent OLED (TOLED) due to its transparency are also used in automotive HUD. A real image can be displayed on the surface of OLED panel as shown in Fig. 1.19. One limitation is the non-transparency of the display background, which may interfere with the driving. Furthermore 
OLED is limited by the low brightness. Although the brightness can be boosted by increasing the power, it will reduce the working life of OLED. As a result, with the current development of the OLED display technology, its application in automotive HUD is limited.
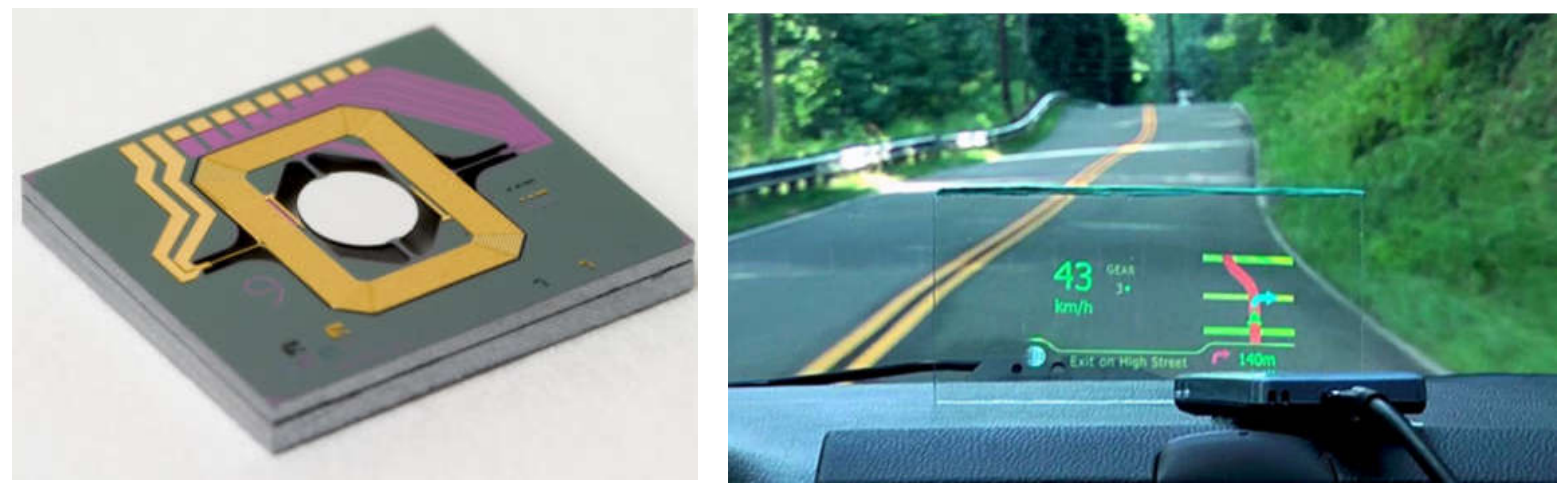

Fig. 1.20 (a) Electromagnetic resonant micromirror [135] (b) HUD based on the micromirror [135].

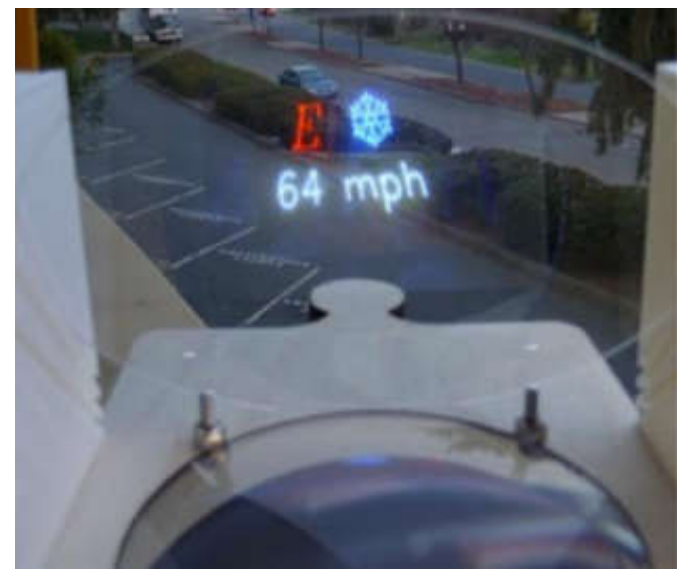

Fig. 1.21 HUD based on the micromirror [122].

LSD has a great potential for automotive HUD application since it shares some of the advantages of DMD and LCoS such as low power consumption, small volume and high display brightness [136]-[138]. Besides it does not suffer from background glow issue. Both raster and vector scanning display HUDs have been developed. Raster scanning HUDs [135], [139], [140] are developed based on a resonant micromirror presented in [9]. The micromirror and the HUD prototype are shown in Fig. 1.20. The light source is provided by lasers diodes with 3 colors, i.e., red, green and blue, which are combined by beam combiner. The micromirror steers the reflected laser beam to scan through a $2 \mathrm{D}$ area line by line. The lasers are modulated to generate colorful displays. Besides of the raster scanning display, vector scanning display is also used in automotive 
HUD application [25], [109], [123]. In automotive HUD application, only simple symbols are required to display the critical driving information which can be showed as vector graphics [141]. In this regard, the vector scanning display HUD is not limited by the lack of abilities to generate pixel image. More important, the vector scanning display HUD requires laser source with much lower power to generate the display with similar brightness as that of raster scanning display. Consequently, the vector scanning display has a potential to be used in automotive HUD. A HUD based on vector scanning is developed in [25] using a 2D quasistatic micromirror. The display result is shown in Fig. 1.21. The micromirror is actuated by vertical comb-drives, which is shown in Fig. 1.15 (a). However, since the comb-drive actuator suffers from "pull-in" instability, it may lead to device failure if exposed to large external vibrations. Another vector scanning display HUD is developed in [109], [123] which use a quasistatic micromirror previously developed by our group [107]. The SEM image of the micromirror and HUD display result are shown in Fig. 1.22. It shares the common advantages of vector scanning display such as low power consumption, small volume and low requirement on the power of laser source to obtain high brightness display. Also, the micromirror can be fabricated using commercial 2-layer surface micromachining MUMPs process, which is mature and low cost compared to the bulk micromachining process used to fabricate vertical comb-drives. Moreover, it is actuated by repulsive-force actuators which do not suffer from the "pull-in" instability and thus has high reliability. However, a small display pattern was obtained attributed to the small optical scanning angle of the micromirror, i.e., $\pm 2.5^{\circ}$ with the aid of the magnification lens. Another issue is that the display has a relative large distortion and is limited to low refreshing frequency i.e., about $30 \mathrm{~Hz}$. Furthermore, the developed display module was not integrated with the car to construct a real HUD system. The first two issues will be solved in objective 2 of the thesis which aims to develop a micromirror based on the repulsive-force actuator developed in objective 1 . Also in objective 2, an advanced display approach will be developed to generate good quality displays in high refreshing frequency. The objective 3 of the thesis is to construct a real HUD system in a car using the micromirror and advanced approach developed in objective 2 . 


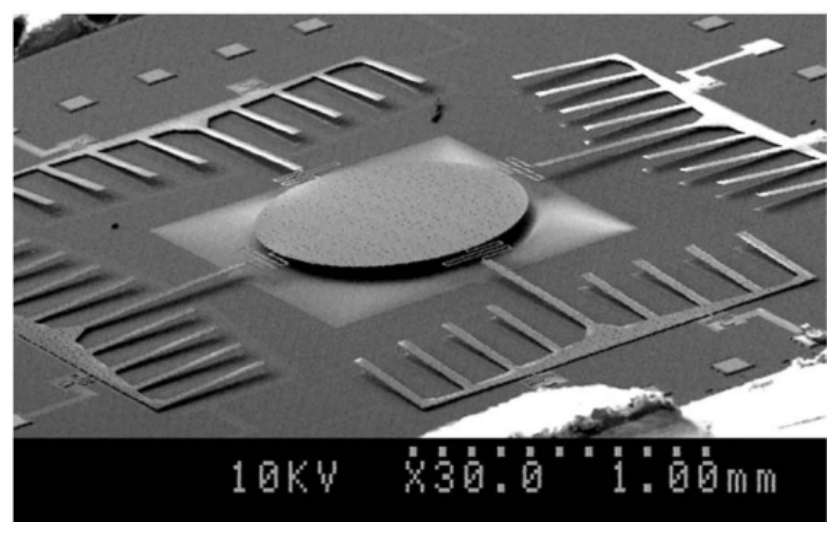

(a)

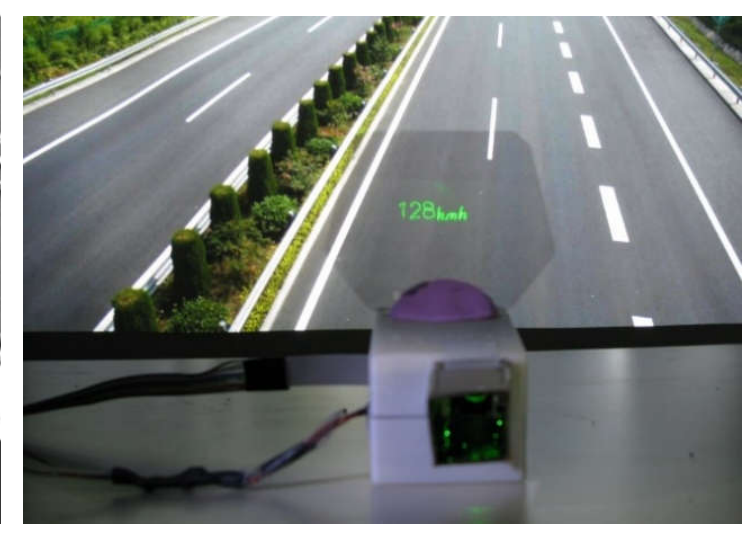

(b)

Fig. 1.22 (a) Micromirror fabricated using surface micromachining process [108] (b) HUD display result using the micromirror [109].

\subsection{Objectives of the thesis}

\section{Objective 1:}

The thesis is to develop repulsive-force rotation actuators which are able to generate larger repulsive force/torque than that of previously reported repulsive-force rotation actuators. The actuators to be developed will share the advantages of common repulsive-force actuators over attractive actuators, i.e., not suffering from the "pull-in" instability and the travel range not limited by the gap between fixed and moving electrodes. In addition, the actuators to be developed will be suitable for simple and matured surface micromachining process. The modeling, fabrication and prototype testing will be conducted on the novel actuators to be developed.

Two actuators are developed in the following two published papers:

- Chao Fan and Siyuan He, "A two-row interdigitating-finger repulsive-torque electrostatic actuator and its application to micromirror vector display", IEEE Journal of Microelectromechanical Systems, vol. 24, pp. 2049 - 2061, 2015.

- Chao Fan and Siyuan He, "A microelectrostatic repulsive-torque rotation actuator with two-width fingers", Journal of Micromechanics and Microengineering, vol. 25, p. 095006, 2015. 


\section{Objective 2:}

The actuators that are developed in objective 1 will be used to drive a 2D scanning micromirror which can be used for LSD. Objective 2 contains two sub-objectives.

Sub-objective 2-1): A 2D scanning micromirror will be developed using the actuator to be developed in objective 1. The micromirror is used for laser vector scanning display. The micromirror is able to solve the issue of small display area and relative large display distortion suffered by the previously reported micromirror.

Sub-objective 2-2): An advanced display approach is to be developed for vector laser display using the micromirror developed in sub-objective 2-1. Compared to the previously reported generic display approach, the advanced display approach is able to achieve better display quality with higher frequency and less display distortion. The advanced display approach is suitable for both the micromirror of this thesis and previously developed micromirrors with improved display quality.

\section{Objective 3:}

The micromirror developed in sub-objective 2-1) is to be used to construct an automotive headup display (HUD), which integrates the micromirror laser and the optics. The HUD will be integrated in the car model in the lab.to form both the real image and virtual image HUDs. 


\section{Chapter 2 Two-row interdigitating-finger repulsive-torque electrostatic actuator}

This chapter is based on the first part of the following published paper:

Chao Fan and Siyuan He, "A two-row interdigitating-finger repulsive-torque electrostatic actuator and its application to micromirror vector display", IEEE Journal of Microelectromechanical Systems, vol. 24, pp. 2049 - 2061, 2015.

This chapter presents a novel two-row interdigitating-finger (TRF) repulsive-torque electrostatic actuator which is able to achieve much larger out-of-plane rotation than the previously developed one-row-finger (ORF) repulsive-force actuators. It meets the first objective of the thesis. The TRF actuator consists of upper and lower rotation units and an output beam. Each unit has one row of rotation fingers, i.e., the lower and upper rotation fingers, which interdigitate with each other. The lower and upper rotation fingers are subject to different electric potentials. Each rotation finger and its underneath aligned fixed finger are subject to the same electric potential. The actuator generates repulsive torques to rotate the lower and upper fingers out-of-plane with the maximum rotation not limited either by the gap between fingers and the substrate nor by the "pull-in" effect. The output beam is connected to the lower row of rotation fingers and therefore the actuator has the same rotation as the lower unit. The actuator requires only two thin films and is suitable for surface micromachining. The model is established to verify the actuator and calculate its performance. Prototypes are fabricated and tested. Experimental results show that the actuator achieved a mechanical rotation of $11.5^{\circ}$ at the static driving voltage of $150 \mathrm{~V}$ and a resonant frequency of $380 \mathrm{~Hz}$ with a sinusoidal driving voltage varying from $20 \mathrm{~V}$ to $70 \mathrm{~V}$.

\subsection{Design}

The two-row interdigitating-finger actuator (TRF) consists of the lower and upper rotation units, and an output beam as shown in Fig. 2.1. Each rotation unit has one row of rotation fingers, i.e., the lower and upper rotation fingers which are interdigitated. The upper unit consists of upper rotation fingers, upper fixed fingers, upper common beam and upper springs. The lower unit consists of lower rotation fingers, lower fixed fingers, lower common beam and lower springs. The 
lower and upper rotation fingers are able to rotate about the lower and upper springs respectively. A middle beam connected to the lower common beam is the output structure which has the same rotation as the lower rotation fingers. The middle beam is connected to a mirror plate for measuring the actuator's performance. The upper rotation unit has two identical parts located on both sides of the middle beam. On each side of every rotation finger there is one interdigitated rotation finger. Each aligned fixed fingers is aligned and underneath the rotation finger. Ideally, all fingers should have the same width. The actuator can be fabricated using surface micromachining process such as PolyMUMPs [112], i.e., Poly0 for lower and upper fixed fingers and Poly1 for lower and upper rotation fingers. In order to meet the fabrication rules of PolyMUMPs and reduce undesired lateral force [97], the rotation fingers are $5 \mu \mathrm{m}$ narrower than the fixed fingers on each side.

The upper rotation and fixed fingers are subject to the electric potential of $V_{l}$, while the lower rotation and fixed fingers are subject to the potential of $V_{2}$. Electrostatic torque is generated on both the lower and upper rotation fingers to rotate them out-of-plane and away from the substrate in opposite direction. The repulsive-force on either the lower or upper rotation finger is the net force by summing the electrostatic forces on the top and bottom surfaces of the finger caused by the asymmetric electric field surrounding them as explained in [33], [35]-[37]. In the interdigitating area, the upper fingers strengthen the electric fields surrounding the lower rotation fingers, which therefore enhance the asymmetric fields caused net forces on the lower rotation fingers, either the upper fingers are lower, the same height or higher than the lower rotation fingers. The lower rotation fingers have the same function to the upper rotation fingers.

The total width and length of the actuator are $1610 \mu \mathrm{m} \times 714 \mu \mathrm{m}$ including pads. The $300 \mu \mathrm{m}$ $\times 300 \mu \mathrm{m}$ mirror plate is connected to the $50 \mu \mathrm{m}$ wide middle beam. The middle beam separates the upper rotation structure into 2 identical parts, each of which has the same dimension $750 \mu \mathrm{m}$ $\times 581 \mu \mathrm{m}$. Rotation fingers are $20 \mu \mathrm{m}$ wide and fixed fingers are $30 \mu \mathrm{m}$ wide. Rotation fingers refer to both the lower and upper rotation fingers. Fixed fingers refer to both the lower and upper fixed fingers. 


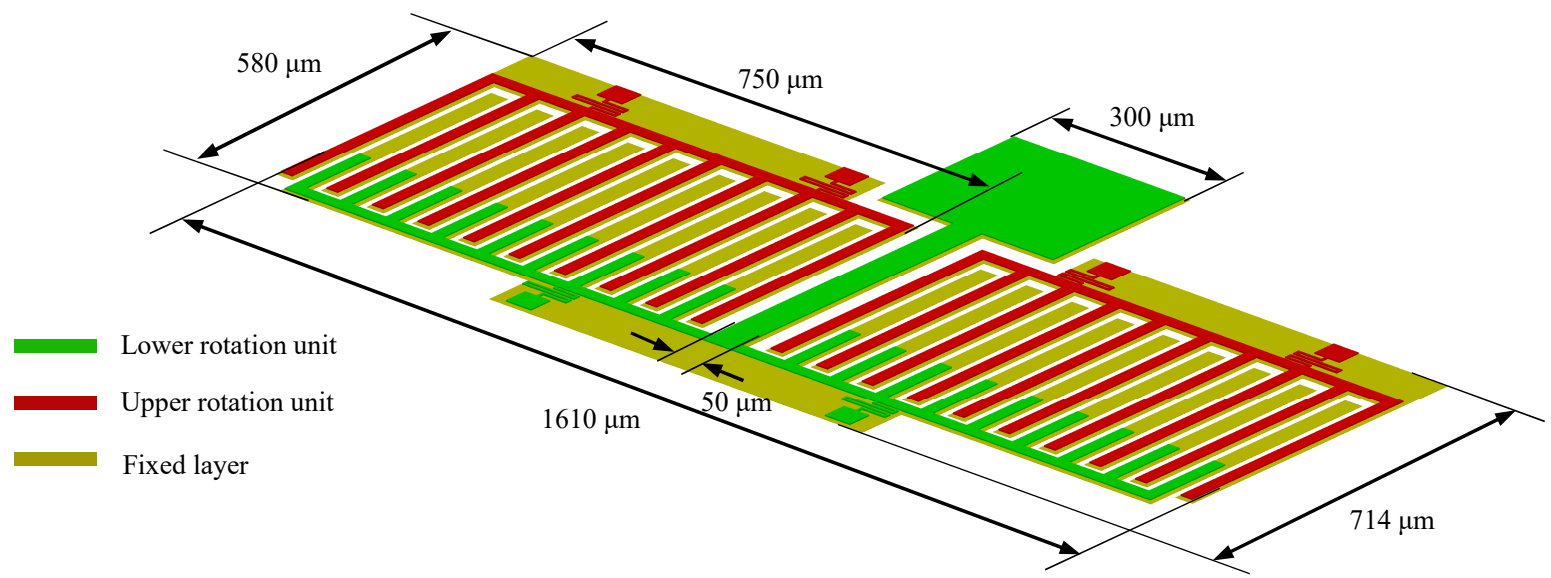

(a)

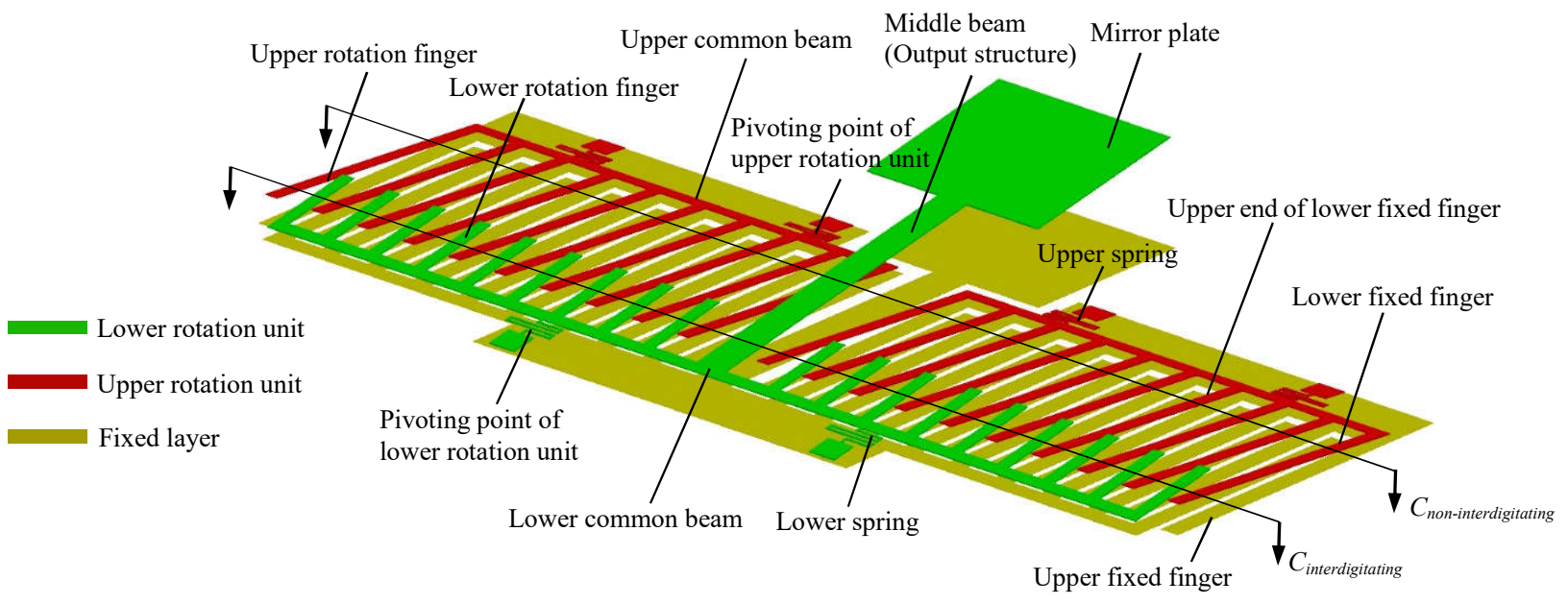

(b)

Fig. 2.1 Two-row interdigitating-finger repulsive-torque electrostatic actuator. (a) Actuator at rest. (b) Actuator in operation.

\subsection{Modeling}

The actuator is modeled to: 1) verify the repulsive-torque is generated and acts on both the lower and upper rotation fingers; and 2) calculate the torques and rotation angles of both the lower and upper rotation fingers. 


\subsubsection{Modeling methodology}

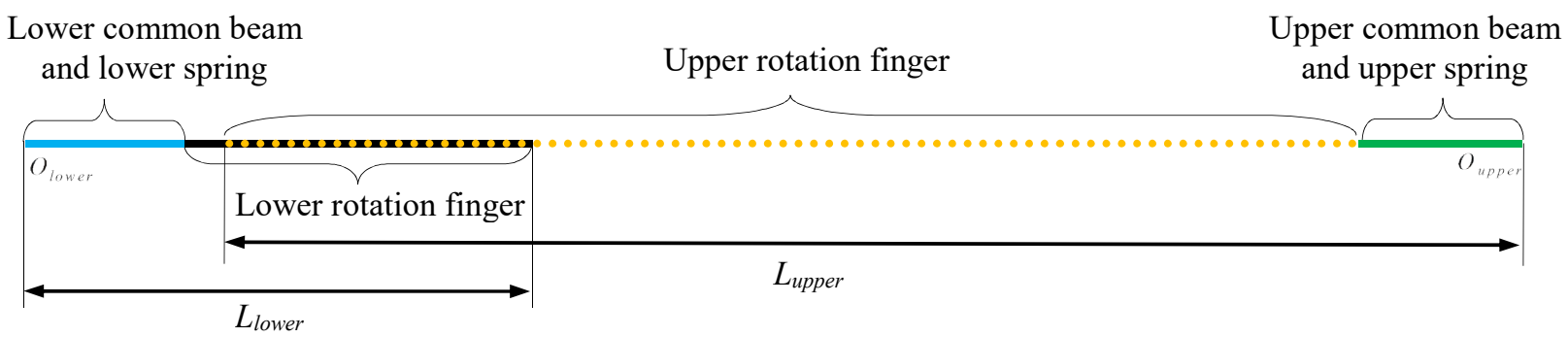

(a)

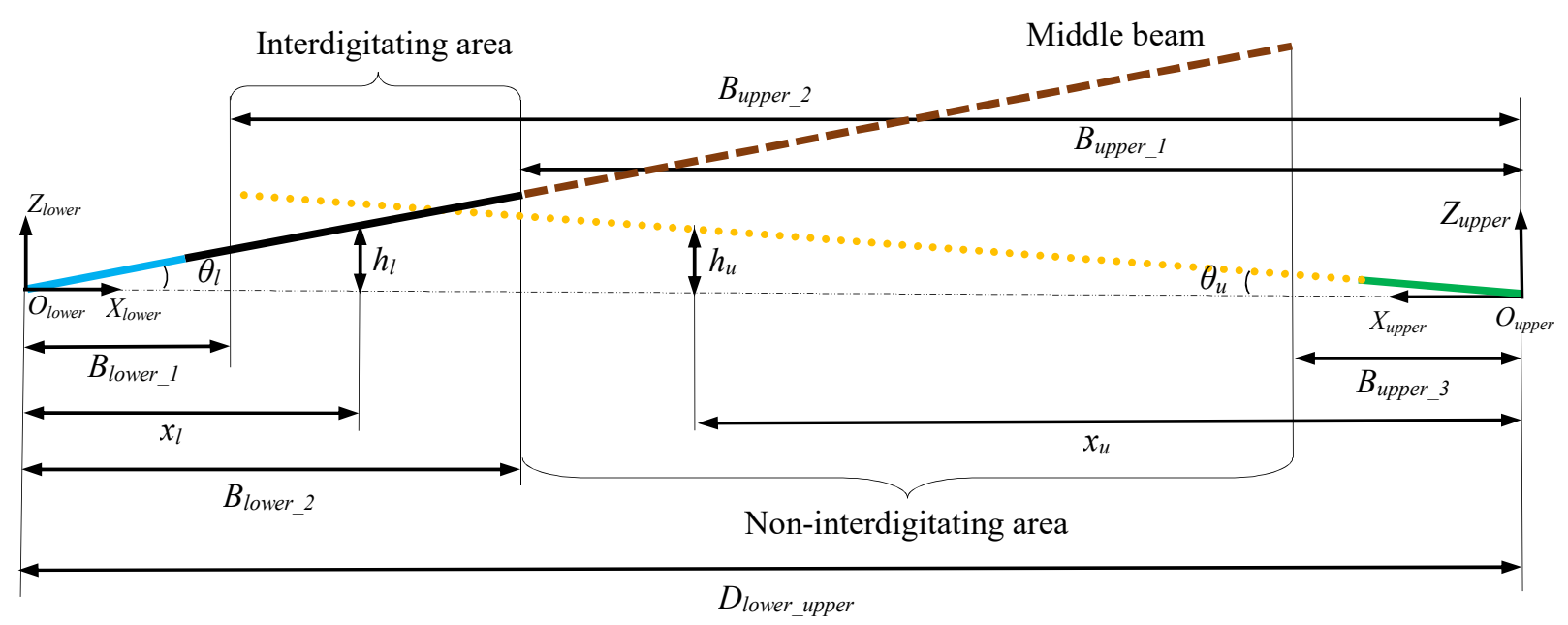

(b)

Fig. 2.2 Side view of interdigitating-fingers of lower and upper rows. (a) Actuator at rest position. (b) Actuator in operation.

Two coordinate systems, i.e., $X_{\text {lower }} O_{\text {lower }} Z_{\text {lower }}$ and $X_{\text {upper }} O_{\text {upper }} Z_{\text {upper }}$ are established with origins $O_{\text {lower }}$ and $O_{\text {upper }}$ located at the lower and upper pivoting points as shown in Figs. 2.1 and 2.2.

A few points are to be explained for Fig. 2.2. The part of the middle beam beyond the tip of the lower fixed finger is not shown in Fig. 2.2 (b) since that part does not generate torque if the fringing effect is not considered. The interdigitating area includes both lower and upper rotation fingers. The non-interdigitating area includes only upper rotation fingers and the middle beam.

The electrostatic torques acting on the lower and upper rotation fingers are balanced by mechanical torques:

$$
\begin{aligned}
& T_{\text {lower }}\left(\theta_{l}, \theta_{u}, V\right)=K_{\text {lower }} \cdot \theta_{l} \\
& T_{\text {upper }}\left(\theta_{l}, \theta_{u}, V\right)=K_{\text {upper }} \cdot \theta_{u}
\end{aligned}
$$


- Where $T_{\text {lower }}$ and $T_{\text {upper }}$ are electrostatic torques acting on the lower and upper rotation fingers.

- Accordiong to the coordianate systems, positive values of $T_{\text {lower }}$ and $T_{\text {upper }}$ indicate that the torques drive the lower and upper rotation fingers to rotate out-of-plane counter-clockwise and clockwise respectively, i.e., the torque is repulsive.

- $K_{\text {lower }}$ and $K_{\text {upper }}$ are rotational stiffnesses of the lower and upper rotation units.

- $\theta_{l}$ and $\theta_{u}$ are roation angles of the lower and upper rotation fingers. $V$ is the driving voltage.

The objective of the modeling is to solve Eq. (2.1) and (2.2) to obtain roation angles of the lower and upper rotation fingers for any given driving voltage $V$. Four steps are followed.

Step 1. Derive functions of torques exerted on lower and upper rotation fingers, i.e., $T_{l o w e r}$ and $T_{\text {upper }}$. The torque functions include expressions of electrostatic forces exerted on lower and upper rotation fingers in cross section with unit length and unit square driving voltage, i.e., $f_{\text {interdigitating_lower, }} f_{\text {interdigitating_upper }}$ and $f_{\text {non-interdigitating_upper. }}$.

Where

$f_{\text {interdigitating_lower: }}$ Acting on the lower rotation fingers in the interdigitating area

$f_{\text {interdigitating_upper: }}$ Acting on the upper rotation fingers in the interdigitating area.

$f_{\text {non-interdigitating_upper: }}$ Acting on the upper rotation fingers in the non-interdigitating area.

Step 2. Obtain functions of $f_{\text {interdigitating_lower, }} f_{\text {interdigitating_upper }}$ and $f_{\text {non-interdigitating_upper }}$ using numerical simulations and polynomial surface fittings.

Step 3. Obtain stiffnesses of the lower and upper rotation units, i.e., $K_{\text {lower }}$ and $K_{\text {upper }}$ using numerical simulation.

Step 4. Solve Eqs. (2.1) and (2.2) by substituting above results into Eqs. (2.1) and (2.2) to obtain roatation angles of the lower and upper roation fingers.

\subsubsection{Modeling}

Step 1. Derive funtions of torques exerted on lower and upper rotation fingers, i.e., $T_{\text {lower }}$ and $T_{\text {upper. }}$. $x_{l}$ is the $X_{\text {lower }}$-axis coordinate of a point on the lower rotation finger. $x_{u}$ is the $X_{u p p e r}$-axis coordinate of a point on the upper rotation finger. The torques are calculated using equations: 


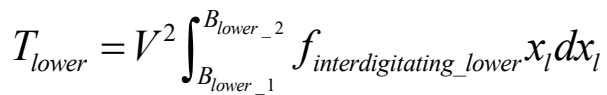

$$
\begin{aligned}
& T_{\text {upper }}=V^{2} \int_{B_{\text {upper_1 }}}^{B_{\text {upper_2}}} f_{\text {interdigitating_upper }} x_{u} d x_{u}+V^{2} \int_{B_{\text {upper__ }}}^{B_{\text {upper }} 1} f_{\text {non-interdigitating_upper }} x_{u} d x_{u}
\end{aligned}
$$

In Eqs. (2.3) and (2.4), the positive values of $f_{\text {interdigitating_lower, }} f_{\text {interdigitating_upper }}$ and $f_{\text {non- }}$ interdigitating_upper indicate the electrostatic forces pointing up and the negative values represent the forces in the downward direction. For any non-unit driving voltage, the electrostatic torque is proportional to $V^{2} . x_{l}$ is the distance between a point on the lower rotation finger to the lower pivoting point $O_{\text {lower. }} . x_{u}$ is the distance between a point on the upper rotation finger to the upper pivoting point $O_{u p p e r} . x_{l}$ and $x_{u}$ are the coordinates of two different points.

$h_{l}$ : In the interdigitating area, $h_{l}$ is the vertical distance between lower rotation and lower fixed fingers as shown in Figs. 2.2 (b) and 2.3 (applicable to $f_{\text {interdigitating_lower }}$ and $f_{\text {interdigitating_upper }}$ ). In the non-interdigitating area, $h_{l}$ is the vertical distance between the middle beam and its underneath lower fixed finger as shown in Fig. 2.2 (applicable to $f_{\text {non-interdigitating_upper) }}$ ).

$h_{u}$ : In both interdigitating and non-interdigitating areas, $h_{u}$ is the vertical distance between upper rotation and upper fixed fingers as shown in Figs. 2.2 (b), 2.3 and 2.5.

In coordinate system $X_{\text {lower }} O_{\text {lower }} Z_{\text {lower }}, h_{l}$ and $h_{u}$ can be expressed as:

$$
\begin{gathered}
h_{l}=x_{l} \cdot \tan \theta_{l} \\
h_{u}=\left(D_{\text {lower_upper }}-x_{l}\right) \cdot \tan \theta_{u}
\end{gathered}
$$

Similarly, in coordinate system $X_{u p p e r} O_{u p p e r} Z_{u p p e r}, h_{l}$ and $h_{u}$ can be expressed as

$$
\begin{gathered}
h_{l}=\left(D_{\text {lower } \_ \text {upper }}-x_{u}\right) \cdot \tan \theta_{l} \\
h_{u}=x_{u} \cdot \tan \theta_{u}
\end{gathered}
$$

Where $D_{\text {lower_upper }}$ is the distance between pivoting points $O_{\text {lower }}$ and $O_{\text {upper }}$. The integration boundary conditions are defined as follows:

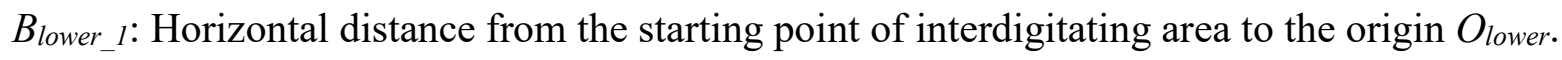
$B_{\text {lower_2: }}$ Horizontal distance from the ending point of interdigitating area to the origin $O_{\text {lower }}$. $B_{\text {upper__ }}$ : Horizontal distance from the ending point of interdigitating area to the origin $O_{\text {upper }}$. $B_{\text {upper__}}$ : Horizontal distance from the starting point of interdigitating area to the origin $O_{\text {upper }}$. 
$B_{\text {upper_3 }}$ : The horizontal distance from the tip of the lower fixed finger to origin $O_{\text {upper, }}$, which is a constant for a given actuator. The integration boundaries can be expressed as:

$$
\begin{gathered}
B_{\text {lower_1 }}\left(\theta_{u}\right)=D_{\text {lower_upper }}-L_{\text {upper }} \cdot \cos \theta_{u} \\
B_{\text {lower_2 }}\left(\theta_{l}\right)=L_{\text {lower }} \cdot \cos \theta_{l} \\
B_{\text {upper_1 } 1}\left(\theta_{l}\right)=D_{\text {lower_upper }}-L_{\text {lower }} \cdot \cos \theta_{l} \\
B_{\text {upper_2 }}\left(\theta_{u}\right)=L_{\text {upper }} \cdot \cos \theta_{u}
\end{gathered}
$$

$L_{\text {lower }}$ : Horizontal distance from $O_{\text {lower }}$ to the tip of lower rotation finger when the actuator is at rest.

$L_{\text {upper }}$ : Horizontal distance from $O_{\text {upper }}$ to the tip of upper rotation finger when the actuator is at rest.

Step 2. Obtain functions of $f_{\text {interdigitating_lower, }} f_{\text {interdigitating_upper }}$ and $f_{\text {non-interdigitating_upper }}$

Functions of $f_{\text {interdigitating_lower, }} f_{\text {interdigitating_upper }}$ and $f_{\text {non-interdigitating_upper }}$ are obtained by numerical simulations and polynomial surface fittings.

Step 2-1 $f_{\text {interdigitating_lower }}$ and $f_{\text {interdigitating_upper }}$

$f_{\text {interdigitating_lower }}\left(h_{l}, h_{u}\right)$ and $f_{\text {interdigitating_upper }}\left(h_{l}, h_{u}\right)$ are forces per unit finger length and unit square driving voltage acting on the lower and upper rotation fingers respectively in the cross sections in the interdigitating area. Dimensions of the fingers and distances between fingers are labeled in Fig. 2.3, which shows only one example of cross sections in the interdigitating area where $h_{u}$ is not necessarily to be smaller than $h_{l}$.

Electro V6.3 [142] software is used to simulate the electrostatic forces in the cross sections using BEM or FEM. The simulation ranges of $h_{l}$ and $h_{u}$ in the interdigitating area are determined using the method introduced in Appendix A as $h_{l}: 0 \sim 50 \mu \mathrm{m}$ and $h_{u}: 0 \sim 50 \mu \mathrm{m}$. The step size of $h_{l}$ and $h_{u}$ is $2 \mu \mathrm{m}$. The simulation results are shown in Fig. 2.4, which are fitted using polynomial surfaces. The surface fittings use two-variable polynomials which are expressed as Eqs. (B1) and (B2) in Appendix B. 


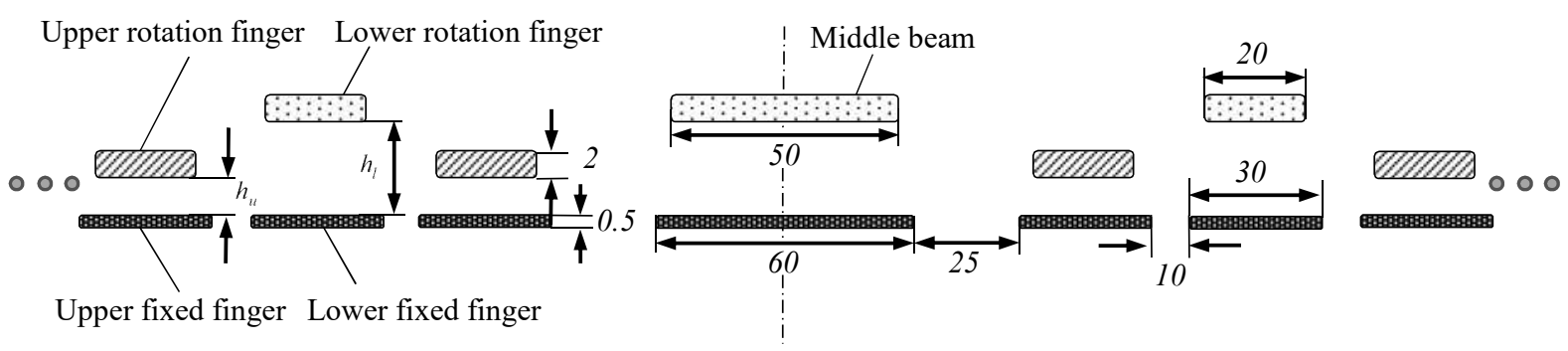

Fig. 2.3 Cross sections of interdigitating area (unit: $\mu \mathrm{m}$ ).

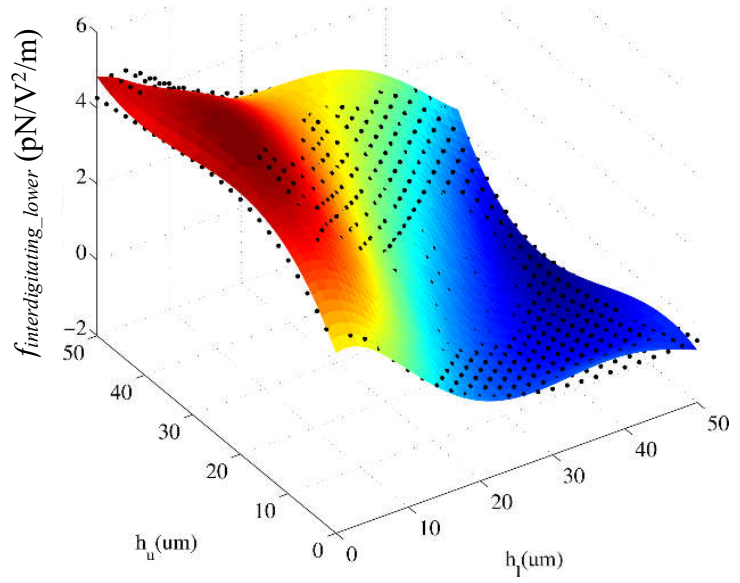

(a)

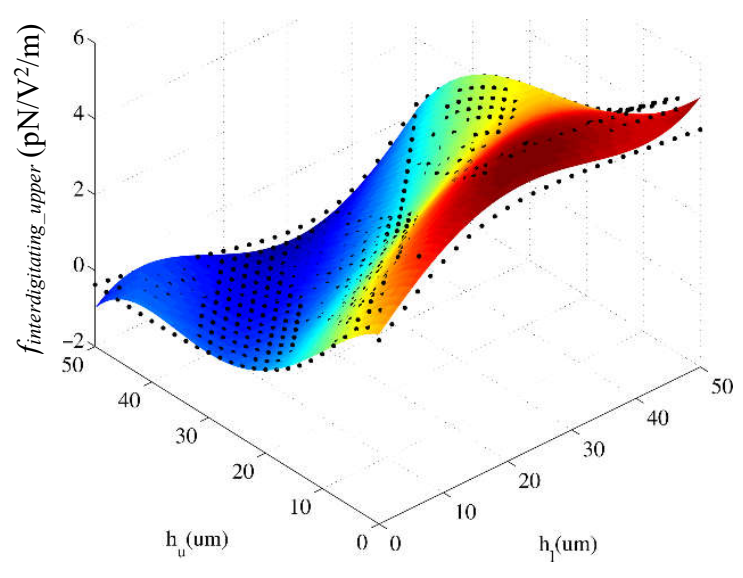

(b)

Fig. 2.4 Simulated and fitting results of electrostatic force in cross sections in the interdigitating

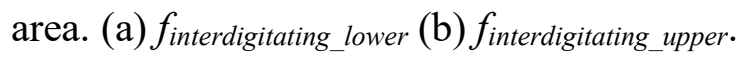

Step 2-2. Obtain function of $f_{\text {non-interdigitating_upper }}$

An example of the cross sections of the non-interdigitating area is shown in Fig. 2.5. The simulation ranges of $h_{l}$ and $h_{u}$ in the non-interdigitating area are determined using the method introduced in Appendix A as $h_{l}: 0 \sim 110 \mu \mathrm{m}$ and $h_{u}: 0 \sim 50 \mu \mathrm{m}$. The step sizes of $h_{l}$ and $h_{u}$ are 2 $\mu \mathrm{m}$. The simulation results and polynomial surface fitting results are shown in Fig. 2.6. The fitting polynomial and its coefficients are shown in Eq. (B3) in Appendix B.
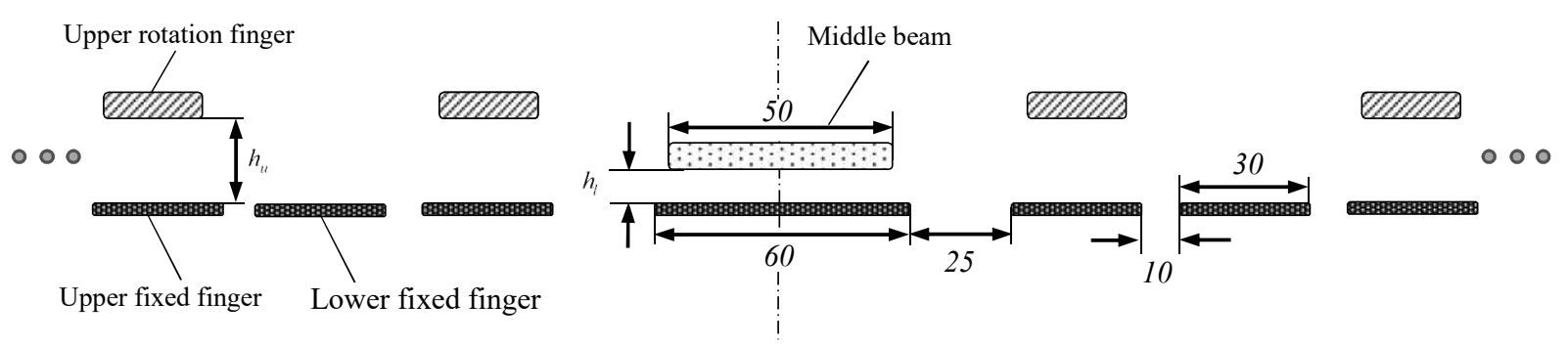

Fig. 2.5 Cross sections of non-interdigitating area (unit: $\mu \mathrm{m}$ ). 
Step 3. Obtain the stiffnesses of the lower and upper rotation units.

The rotational stiffnesses of the lower and upper unit, $K_{\text {lower }}$ and $K_{\text {upper }}$, are obtained through FEM simulations which are $3.4 \times 10^{-9} \mathrm{Nm} / \mathrm{rad}$ and $8.5 \times 10^{-9} \mathrm{Nm} / \mathrm{rad}$ (sum of the stiffnesses of 2 identical parts).

Step 4. Solve Eqs. (2.1) and (2.2) to obtain roatation angles of the lower and upper roation fingers, i.e., $\underline{\theta_{l}}$ and $\theta_{u}$.

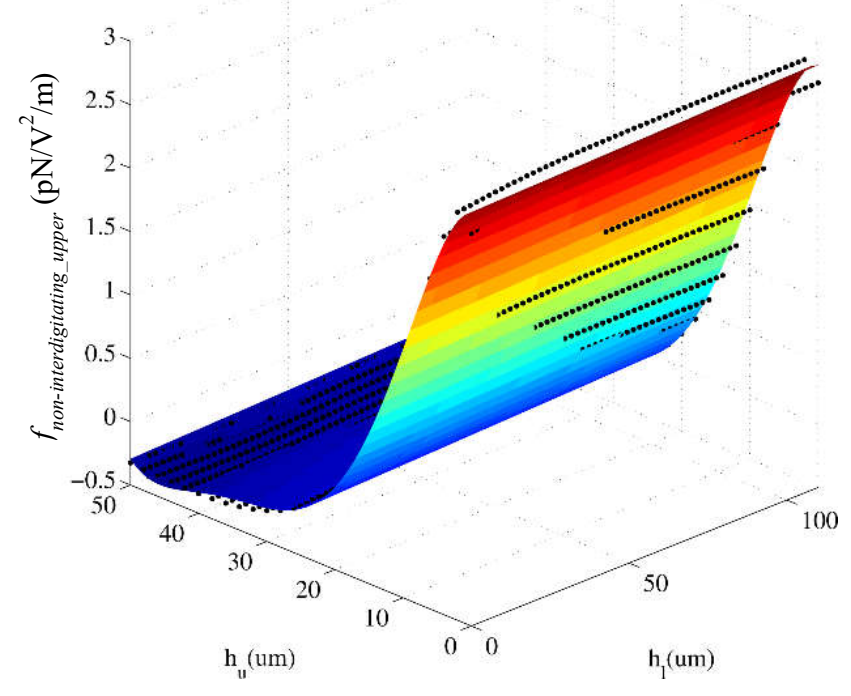

Fig. 2.6 Simulated and fitting forces in cross sections of non-interdigitating area.

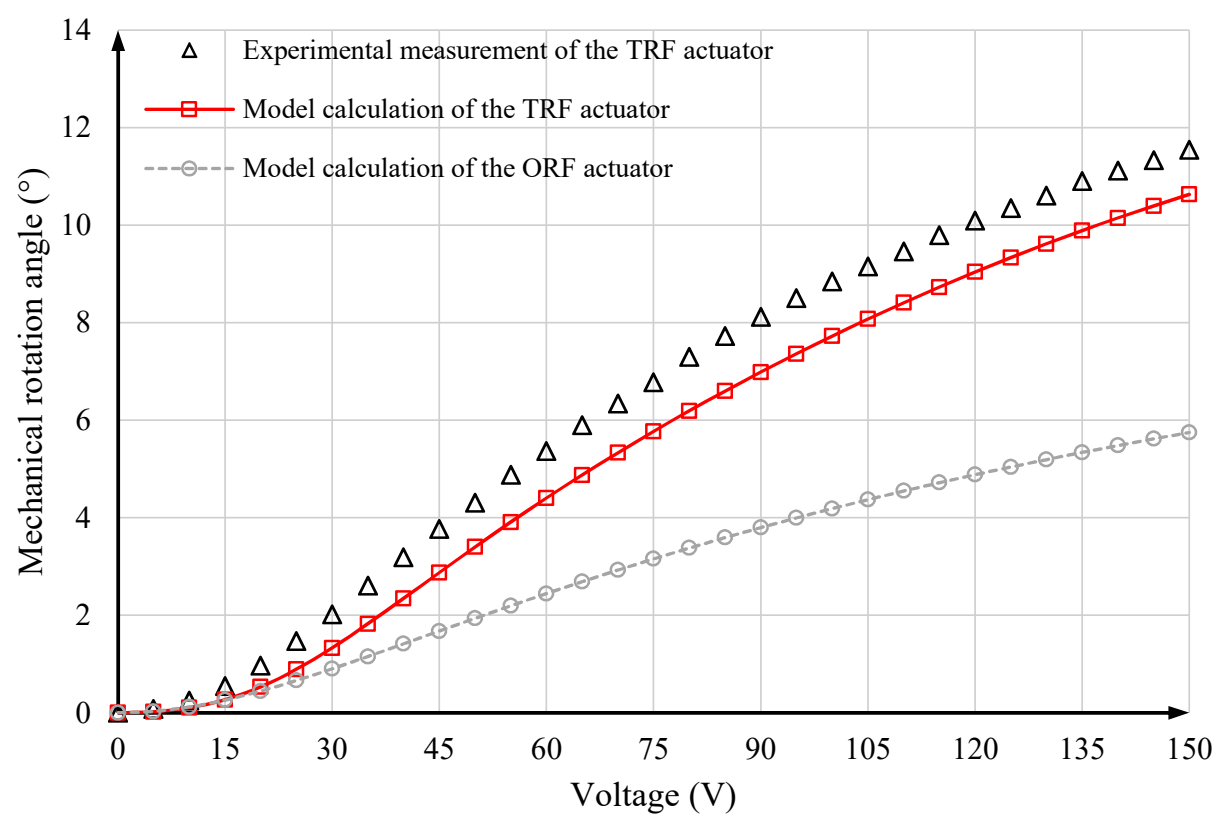

Fig. 2.7 Rotation angles of the actuators. 


\subsection{Performance evaluation}

\subsubsection{Rotation angle estimation and comparison with one-row-finger (ORF) actuator}

Substituting results of Step $1 \sim 3$ into Eqs. (2.1) and (2.2) there are only two unknowns i.e., $\underline{\theta}_{l}$ and $\theta_{u}$, which can be solved. $\theta_{l}$ can be obtained as shown in Fig. 2.7. The actuator torque, $T_{l o w e r}$ is calculated using Eq. (2.3) with the results shown in Fig. 2.8. The rotation angle of the upper rotation fingers, $\theta_{u}$ and the torque Tupper (Eq. (2.4)) can also be obtained with the results shown in Fig. 2.9.

The modeling results show that both the torques exerted on lower and upper rotation fingers are positive. Even when there are small negative forces in some cross sections as shown in Figs. 2.4 and 2.6, the torques obtained by integrating the multiplications of the forces in all cross sections and the corresponding distances are positive. According to the coordinate systems established for modeling, positive values of the torques indicate that the torques rotate the lower and upper fingers out-of-plane and away from the substrate counter-clockwise and clockwise respectively, i.e., repulsive. The output structure of the actuator, i.e., the middle beam, is connected to the lower common beam and has the same rotation as the lower rotation fingers, i.e., $\theta_{l}$. Thus the actuator generates repulsive-torque and outputs out-of-plane rotation. The model predicts that the actuator is able to output an out-of-plane mechanical rotation of $10.63^{\circ}$ at $150 \mathrm{~V}$ as shown in Fig. 2.7.

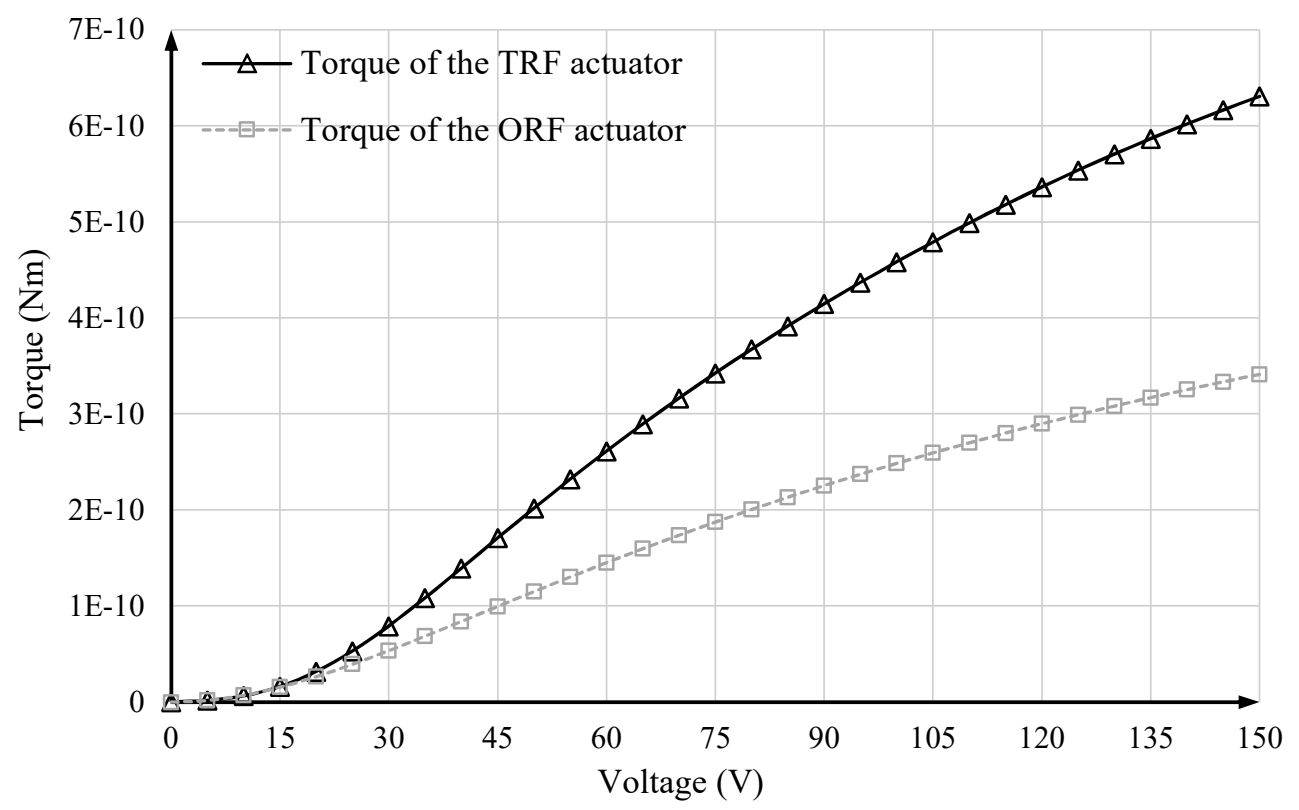

Fig. 2.8 Torque of the actuator. 


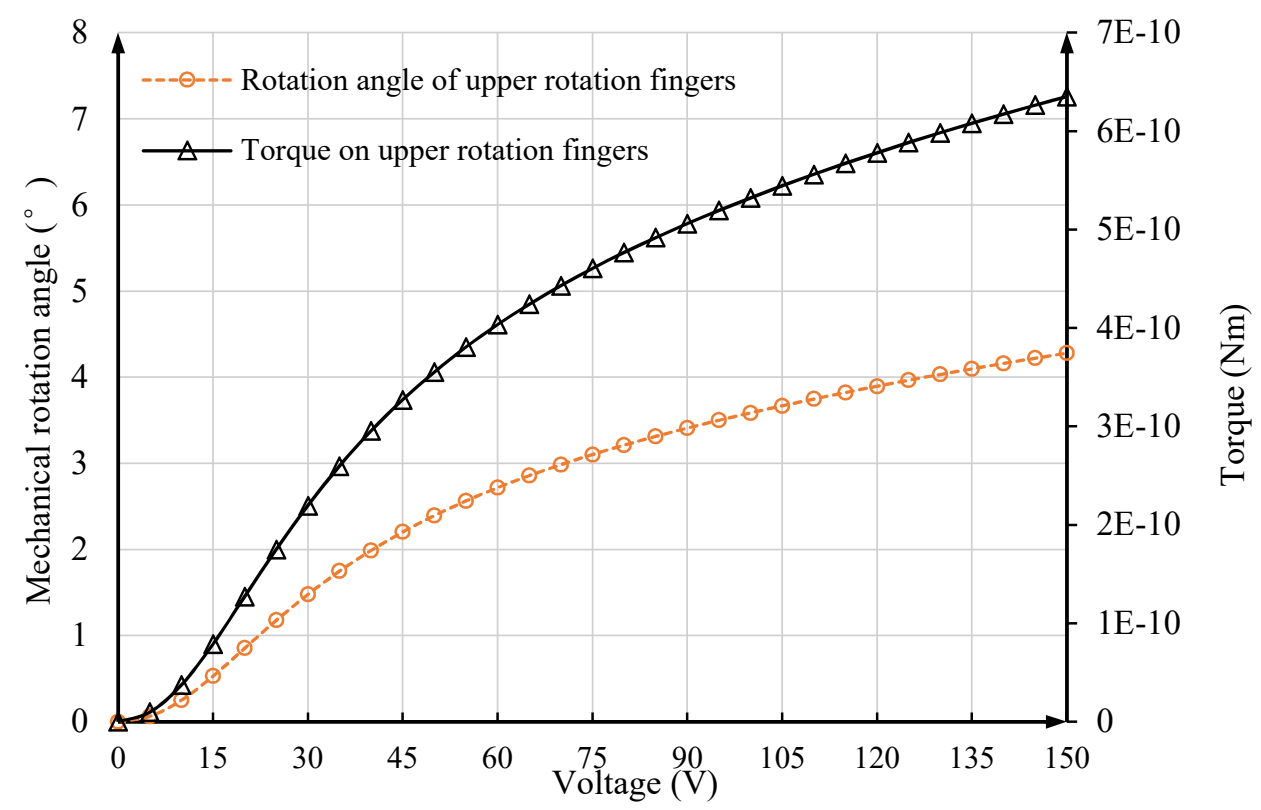

Fig. 2.9 Torque and rotation angle of the upper rotation fingers.

The two-row interdigitating-finger repulsive-torque actuator developed in this chapter is compared to the previously reported ORF rotation actuator [97], [105], which is designed using the same area, stiffness and driving voltage of the actuator developed in this chapter following the optimization method presented in [105]. The comparison results show that the TRF actuator developed in this chapter can generate a much higher torque and then a much larger rotation angle than that of the ORF actuator, e.g., the improvement is $75 \%$ at $50 \mathrm{~V}, 84.2 \%$ at $100 \mathrm{~V}$ and $84.5 \%$ at $150 \mathrm{~V}$ respectively.

The model developed in the previous section are also used to study the effect of the total length of the actuator to its static performance using the model developed in the previous sections. There are some conditions of this study:

(1) The finger width is fixed to be $20 \mu \mathrm{m}$ which is the same as the actuator presented in above sections.

(2) The total width of the actuator stays unchanged which is $1610 \mu \mathrm{m}$

(3) The ratio of the lower and upper finger is fixed to be 3 which is the same as the actuator presented above. It is noted that the length of the finger is counted from the fingertip to the root of the finger where it connects with the common beam. 
(4) The dimensions of all the springs, the pad and the common beams are the same as the actuator presented above.

The finger lengths of the actuator presented in the previous section is used as a reference. A serious of scale factors from 0.2 to 1.2 with 0.2 increment are used to scale the finger lengths of both lower and upper fingers. The total length of the actuator changes with the finger lengths For comparison purpose, the performance of previously developed one-row-finger actuator (ORF) of the same length as each variant of the TRF actuator is evaluated. Different from TRF actuator whose finger width is fixed to be $20 \mu \mathrm{m}$, the finger width of the ORF actuator is optimized using the method presented in [105] to generate maximum out-of-plane rotation at $150 \mathrm{~V}$.

The calculated rotation angles of ORF actuator and two-row-finger actuator are plotted in Fig. 2.10. The rotation angles of TRF and ORF actuators verse the driving voltages are denoted as black solid and grey dotted curves respectively. It can be concluded that the rotation angle of both the ORF and TRF actuators increase with the increase of the actuator length. The increase of the rotation angle of the TRF actuator is much more than that of the ORF actuator. It can be seen that the rotation angles of the ORF are clustered together which indicate that the increase of the total length of the actuator does not lead to significant improvement of the rotation angles. The curves of the TRF actuator are all above that of the ORF actuator except when the scale is smaller than 0.4 (the total length of the actuator is smaller than $430 \mu \mathrm{m}$ ). This is because that the dimensions of the pad and the springs are constant and when the total length of the actuator is small, the dimensions of the upper pads and upper springs are comparable to the total length of the actuator and they cannot generate out-of-plane torque. While the area taken by the upper pads and springs are used to generate out-of-plane torque by the ORF actuator. However, it is worth noting that for some applications such as micromirror the total length of the actuator is usually large and the TRF actuator is always superior over the ORF actuator. 


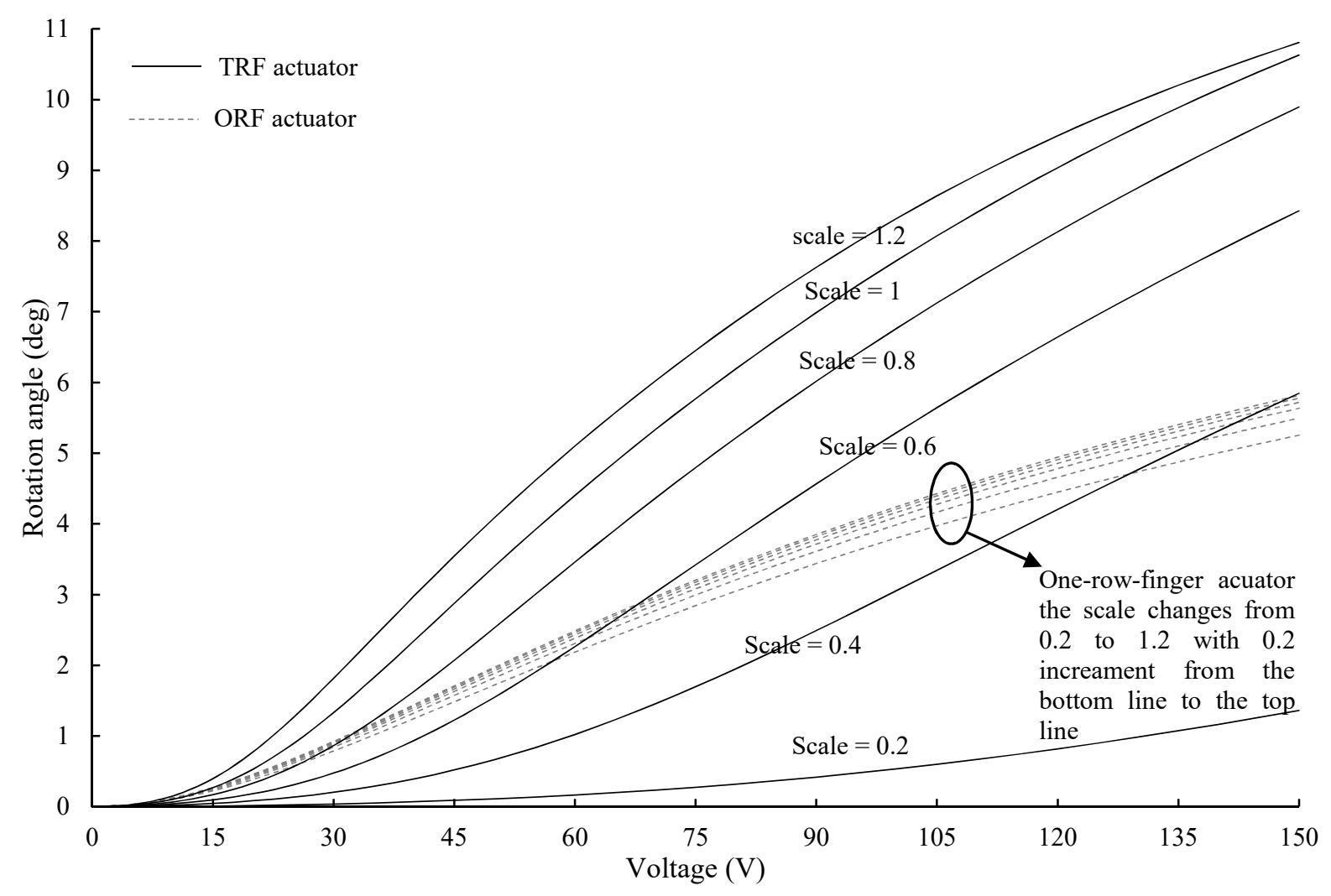

Fig. 2.10 Rotation angles of TRF actuator and ORF actuator at various actuator lengths.

\subsubsection{Dynamic response estimation by numerical simulation}

Since there are two rotation units in the two-row finger actuator presented in this chapter, the resonant frequency of the actuator $\left(f_{\text {actuator }}\right)$ is the smaller value among the resonant frequency of lower rotation unit $\left(f_{\text {lower }}\right)$ and the resonant frequency of the upper rotation unit $\left(f_{\text {upper }}\right)$. Generally the actuator is connected to an operation structure through its lower row of rotation fingers, e.g., a 1D micromirror plate such as the design in Sections II IV, or a $2 \mathrm{D}$ micromirror plate such as the design in Section V. Subsequently the lower rotation unit has high moment of inertia and then a low resonant frequency, i.e., much lower than that of the upper rotation unit. Thus $f_{\text {actuator }}$ is normally equal to $f_{\text {lower }}$. The lower rotation unit includes lower springs, lower rotation fingers, lower common beam, the middle beam and the operation structure such as the mirror plate. In the actuator in Sections II VI, the lower rotation unit has the resonant frequency of $557 \mathrm{~Hz}$ with the resonant mode shown in Fig. 2.11 and the upper rotation unit has the resonant frequency of 1.59 KHz. It is noticed that the middle beam has a slight deformation, i.e., the beam bends along its 
length relative to its root end connecting the lower common beam due to the large moment of inertia of the mirror plate attached to the middle beam.

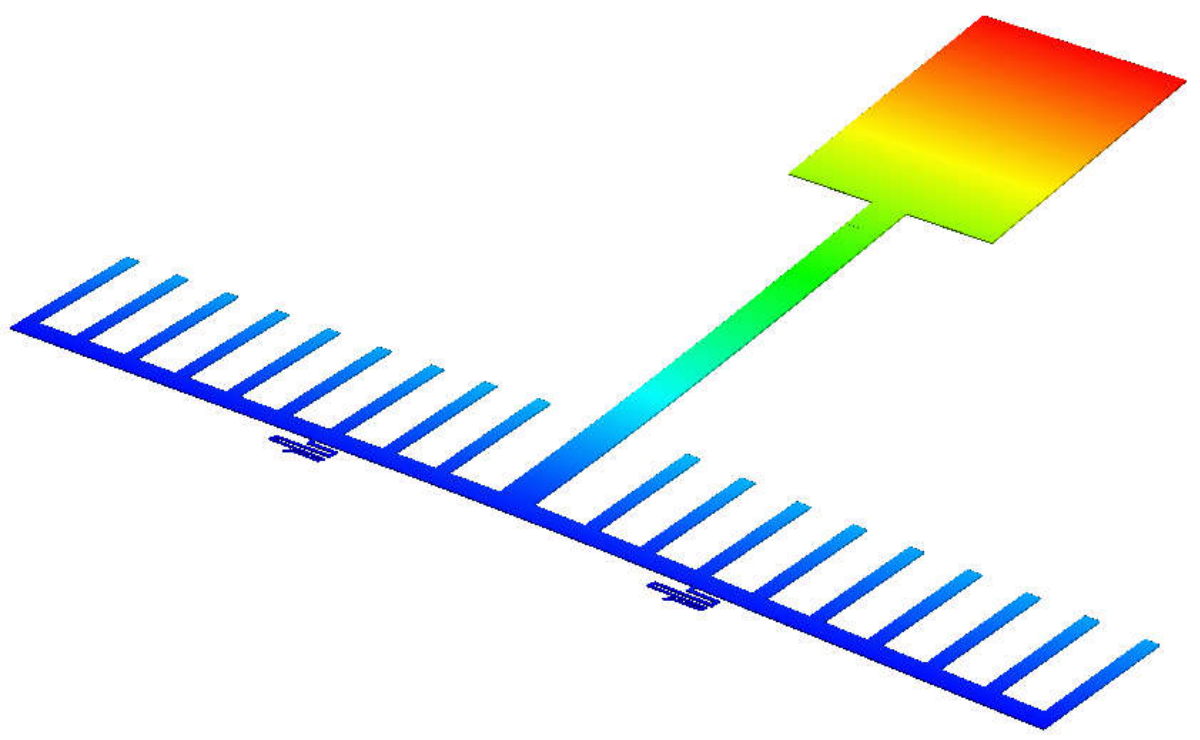

Fig. 2.11 Resonant modes of actuator.

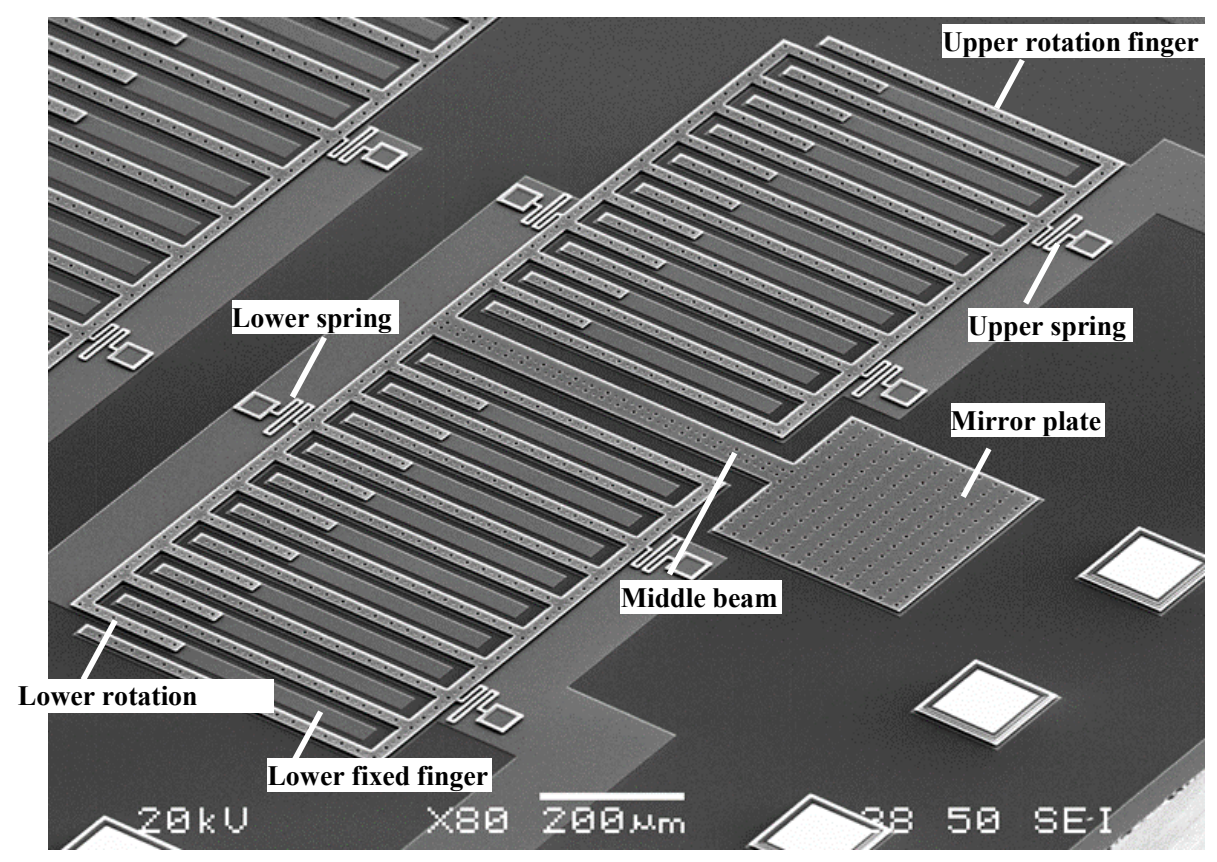

(a) 


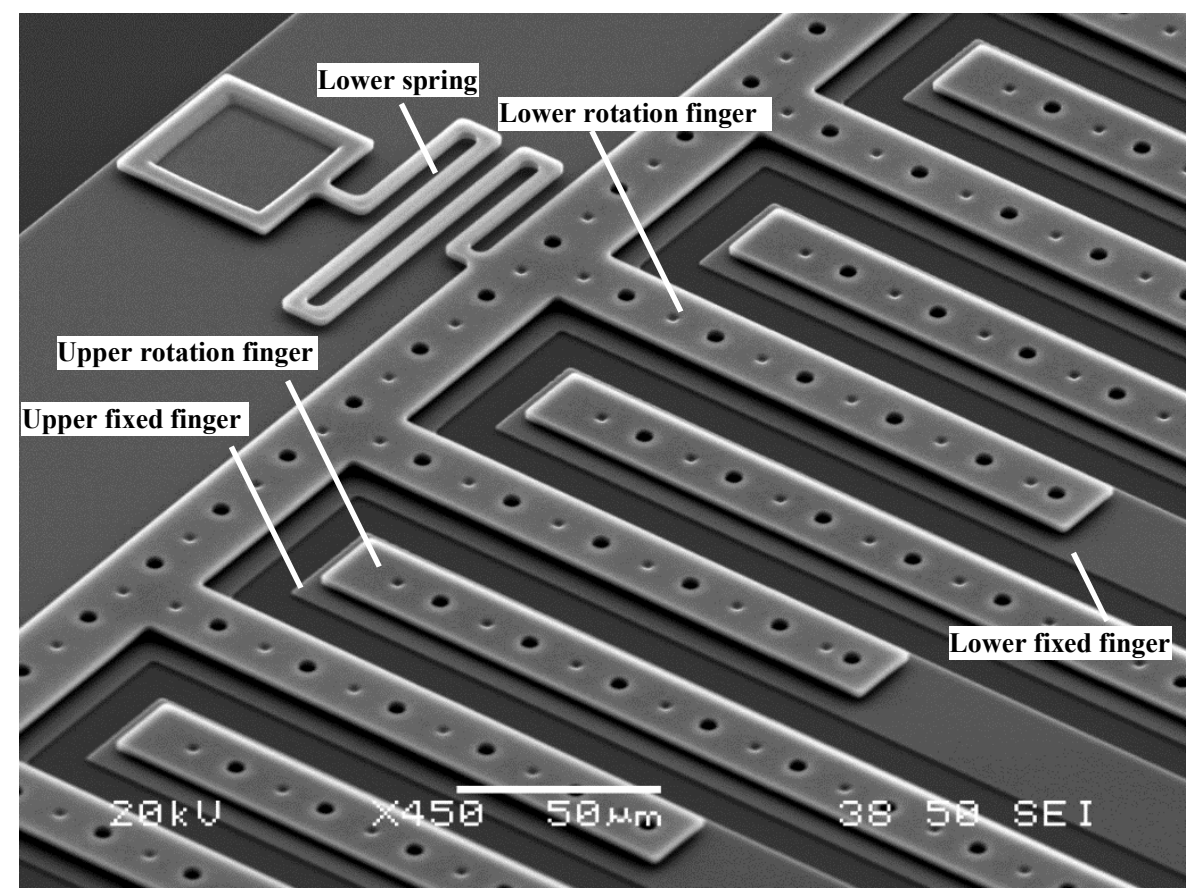

(b)

Fig. 2.12 SEM images of the prototype. (a) The actuator (b) Lower spring, lower and upper fingers.

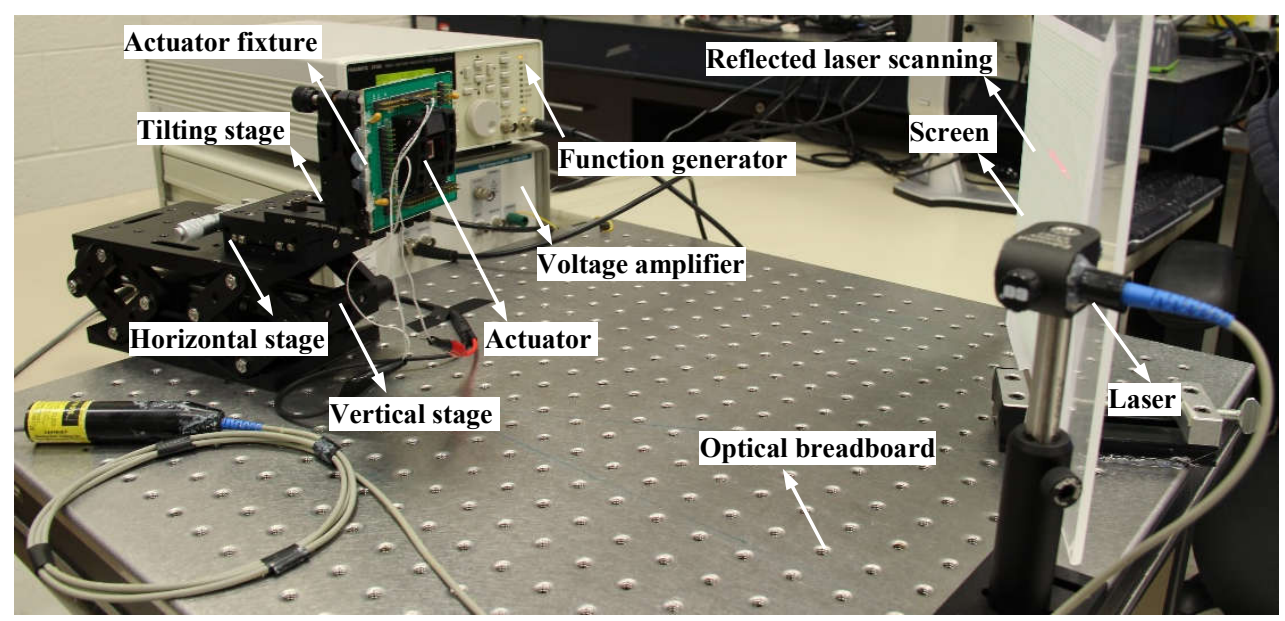

Fig. 2.13 Experiment setup for measuring the rotation of the actuator.

\subsection{Prototype and performance testing}

The prototype is fabricated using PolyMUMPs, as shown in Fig. 2.12. Both static and dynamic performances of the prototypes are measured. The testing setup is shown in Fig. 2.13. A laser beam shoots onto the mirror plate attached to the middle beam. The actuator is packaged in a CQFP44 chip and is mounted on an actuator fixture which is installed on one tilting stage and two linear translation stages. A display screen is attached with a grid paper. The stages are to ensure: 1) the 
mirror plate is perpendicular to the optical bench; and 2) the incident and reflected laser beam are parallel to the optical bench. A function generator and a voltage amplifier are used to drive the actuator.

\subsubsection{Static performance}

Static performance refers to the rotation angles of the actuator when subjected to different DC voltages. In the test, $\mathrm{DC}$ voltages from 0 to $150 \mathrm{~V}$ with $5 \mathrm{~V}$ increment are applied to the actuator. The actuator steers the reflected laser beam and changes the location of the laser spot on the screen as it rotates. The optical and then mechanical rotation of the actuator is calculated by measuring the displacement of the laser spot on the display screen.

In order to relate the tilt angle of the mirror plate with the laser spot displacement on the display screen it is necessary to convert the $3 \mathrm{D}$ optical geometry into a $2 \mathrm{D}$ problem. The schematic top view of the optical geometry is shown in Fig. 2.14. To convert the 3D optical geometry into a 2D problem, the virtual plane composed of the incident laser beam and the reflected laser beam needs to be guaranteed to be parallel to the optical breadboard. It only needs to be guaranteed that two lines on the plane, i.e., $O O_{1}$ and $O_{1} O_{2}$ are parallel with the optical breadboard separately.

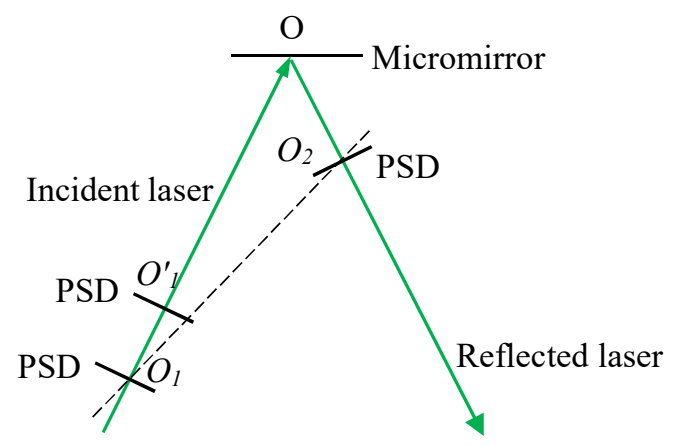

Fig. 2.14 Schematic top view of the optical geometry.

First, line $O O_{1}$ is considered to be parallel to the optical breadboard by the optomechanical components used to fix the laser. This is verified by measuring the angle between $O O_{1}$ and the optical breadboard by using the Position Sensing Detector (PSD). The PSD is used because it can measure the tiny shift of the laser spot on its active sensing area. The schematic setup of measuring the incident beam angle with respect to the optical breadboard is shown in Fig. 2.15. As shown in Fig. 2.15, the PSD is positioned in at two different locations in front of the incident laser path, $O_{1}$ and $O^{\prime}{ }_{1}$. Line $O O_{1} O_{1}^{\prime}$ is the projection line of the actual beam path of $O A B$ and is parallel to the 
optical breadboard. The actual laser path $O A B$ is assumed to have an inclination angle $\alpha$ with $O O_{1} O^{\prime}{ }_{1}$, which can be calculated by Eq. (2.13).

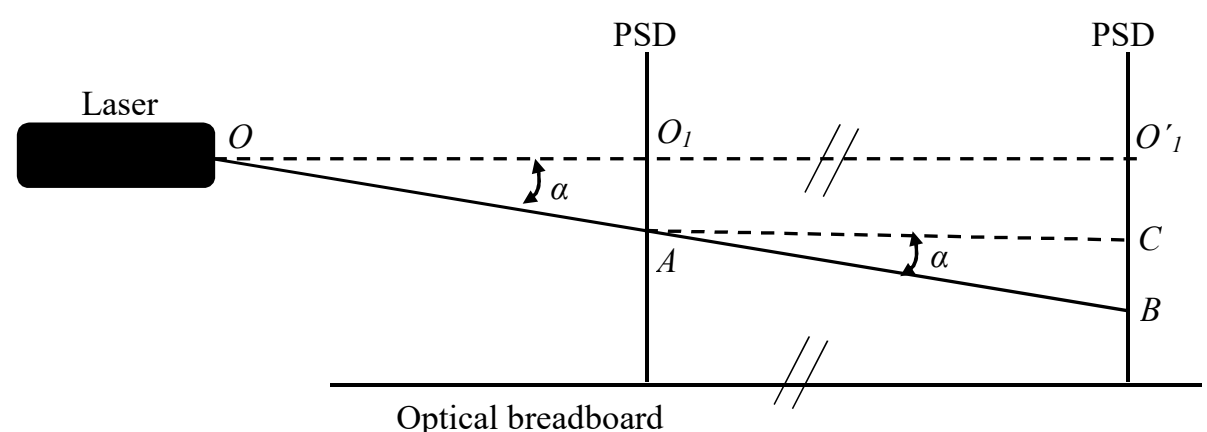

Fig. 2.15 Schematic side view of verifying the angle between the incident laser beam and the optical breadboard.

$$
\alpha=\arctan \left(\frac{B C}{O_{1} O_{1}^{\prime}}\right)
$$

Where $B C$ is the vertical displacement of the laser spot measured by PSD by moving it from location $A O_{1}$ to $B^{\prime}{ }_{2} . O_{1} O_{1}^{\prime}$ is the distance between the two locations of the PSD. The angle $\alpha$ is calculated to be $0.4^{\circ}$ which is a small enough value to consider the incident laser beam to be approximately parallel with the optical breadboard. The vertical position of the laser spot $B$ on the PSD is recorded in position $A O_{1}$.

Then it needs to be guaranteed that line $\mathrm{O}_{1} \mathrm{O}_{2}$ is also parallel with the optical breadboard. Then the PSD is moved to the reflected laser path $\mathrm{O}_{2}$ the vertical position of the laser spot on the PSD is denoted as $B^{\prime}$. If vertical positions of $B$ and $B^{\prime}$ are the same, it can be improved that $O_{1} O_{2}$ is also parallel with the optical breadboard. However, due to assembly and installation error, the plane of the micromirror plate is not perpendicular to the optical breadboard and as a result the vertical position of $B^{\prime}$ and $B$ are different at this time. The tilting stage as shown in Fig. 2.13 is used to tune the pitch angle of the test fixture so that $B^{\prime}$ has the same vertical positions as that of $B$. At this point the line $\mathrm{O}_{1} \mathrm{O}_{2}$ is tuned to be parallel with the optical breadboard and furthermore the plane constructed of the incident and reflected laser beam is also parallel with the optical breadboard. As a result, the 2D geometry shown in Fig. 2.16 can be used to analyze the relationship of the displacement of the laser beam on the screen (shown in Fig. 2.12) with the rotation angle of the actuator. 


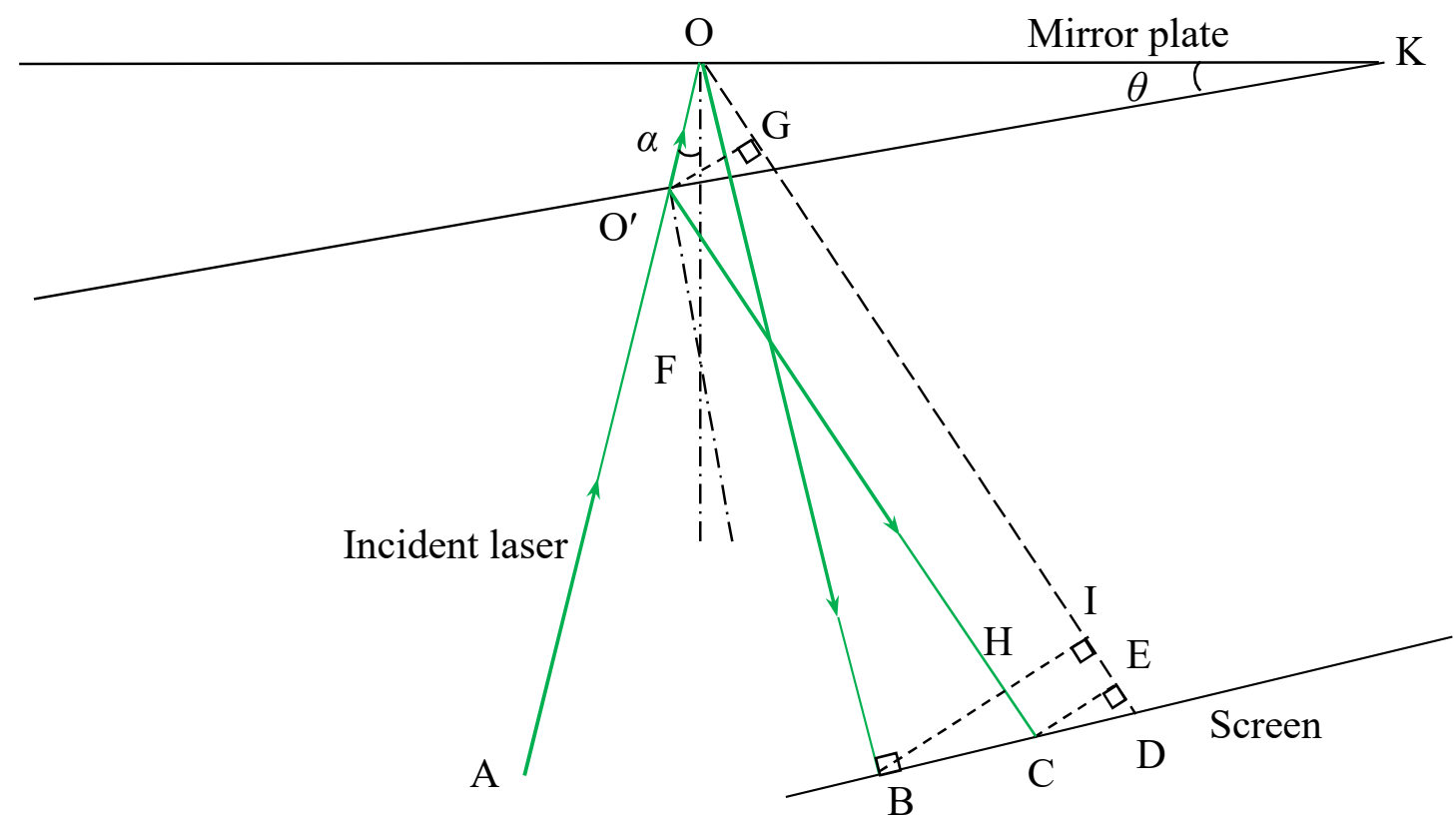

Fig. 2.16 Schematic top view of the geometry relationship between the tilt angle of the mirror plate and the displacement of the laser spot on the screen.

In Fig. 2.16, line $A O$ is the incident laser beam and intersect with the mirror plate at point $O$ when no driving voltage is applied to the actuator. Line $O B$ is the reflected laser beam and intersect with the paper screen at point $B . O F$ is the normal line of the mirror plate when it is at rest position. The incident angle is $\alpha$. The incident laser beam $A O$ intersect with the mirror plate at point $O^{\prime}$ after the mirror rotate an angle of $\theta$ from its rest position. Line $O^{\prime} F$ is the normal line of the mirror plate at the tilted position. At the tilted position the laser is reflected along line $O^{\prime} C$ and intersects with the display screen at point $C . O D$ is a construction line which is parallel with $O^{\prime} C$ and intersect with the paper screen at point $D . O^{\prime} G$ is a construction line which is perpendicular with $O D$. $C E$ is the construction line which is parallel with $O^{\prime} G$ and they have equal length. It can be proved that in the triangular $\triangle O B D$, the angle $\angle B O D$ is equal to twice of the rotation angle of the mirror plate, i.e., $2 \theta$. In this case, $\theta$ can be calculated using Eq. (2.14):

$$
\theta=\frac{1}{2} \arctan \left(\frac{B D}{O B}\right)
$$

However in Eq. (2.14), BD cannot be measured directly. Alternatively, BD can be proved to be approximately equal to BC with negligible error. It can be proved from Eq. (2.15) (2.18) that if the radius of the mirror, i.e., $r$, plate is much smaller than the distance from the center of the 
mirror plate to the display screen and the incident laser angle $\alpha$ is relatively small $B C$ and $B D$ can be considered close enough so that $B C$ can replace the value of $B D$ in Eq. (2.14).

In the experiment, the radius of the mirror plate is $0.15 \mathrm{~mm}, O B$ is $340 \mathrm{~mm}$ and $\alpha$ is $13.75^{\circ}$. In this case, the maximum ratio of $B C$ with $B D$ is $99.97 \%$. This means it is reasonable that $B C$ can be used as an approximate value of $B D$.

$$
\begin{gathered}
\frac{B C}{B D}=\frac{B H}{B I} \\
H I=O^{\prime} G=\frac{\sin \theta}{\cos (\alpha+\theta)} \cdot r \\
B I=\sin (2 \theta) \cdot O B \\
\frac{B C}{B D}=\frac{B H}{B I}=\frac{B I-H I}{B I}=1-\frac{1}{\cos (2 \theta+\alpha)+\cos \alpha} \cdot \frac{r}{O B} \leq 1-\frac{1}{2 \cos \alpha} \cdot \frac{r}{O B}
\end{gathered}
$$

In this case, the rotation angle of the mirror plate can be calculated by measuring the displacement on the grid paper using Eq. (2.19):

$$
\theta=\frac{1}{2} \arctan \frac{B C}{O B}
$$

The measured static performance of the actuator is shown in Fig. 2.7. Multiple actuators $(\geq 3)$ are measured under the same setup and it has been proved that the rotation angles are consistent with different actuators fabricated in the same run. The experimental test shows that the two-row interdigitating-finger repulsive-torque actuator can achieve a mechanical rotation angle of $11.5^{\circ}$ at $150 \mathrm{~V}$. The discrepancy between the model results and the experimental results could be attributed to that the model does not consider the electrostatic torques generated between the common beams and the finger tips.

\subsubsection{Dynamic performance}

Different from the static display which uses a display screen to manually record the displacement of the laser spot, a PSD is used in the dynamic performance test, which is able to measure the laser spot displacement on its sensing area. The reason that PSD is not used for static measurement is that large voltages $(0 \sim 150 \mathrm{~V})$ is applied to the actuator which lead to a large laser 
displacement. Due to the small size of the sensing area of PSD which is $10 \mathrm{~mm} \times 10 \mathrm{~mm}$, it cannot fit it the large displacement no matter how close the PSD positioned to the actuator. However for dynamic test, the dynamic behavior of the actuator cannot captured by display screen. As a result, a relatively smaller peak to peak driving voltage $(20 \sim 70 \mathrm{~V})$ compared to the ones (e.g., $0 \sim 150$ V) used in static test is applied to the actuator in dynamic performance tests, to ensure the laser scanning area is smaller than the PSD sensing area.

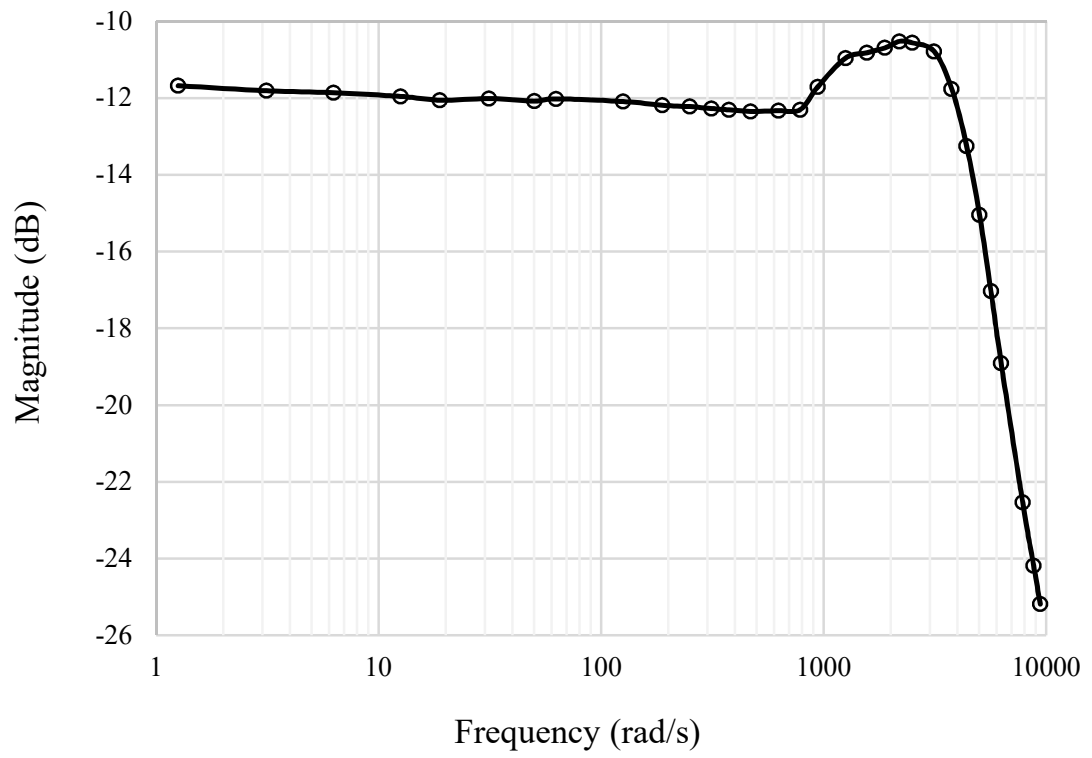

(a)

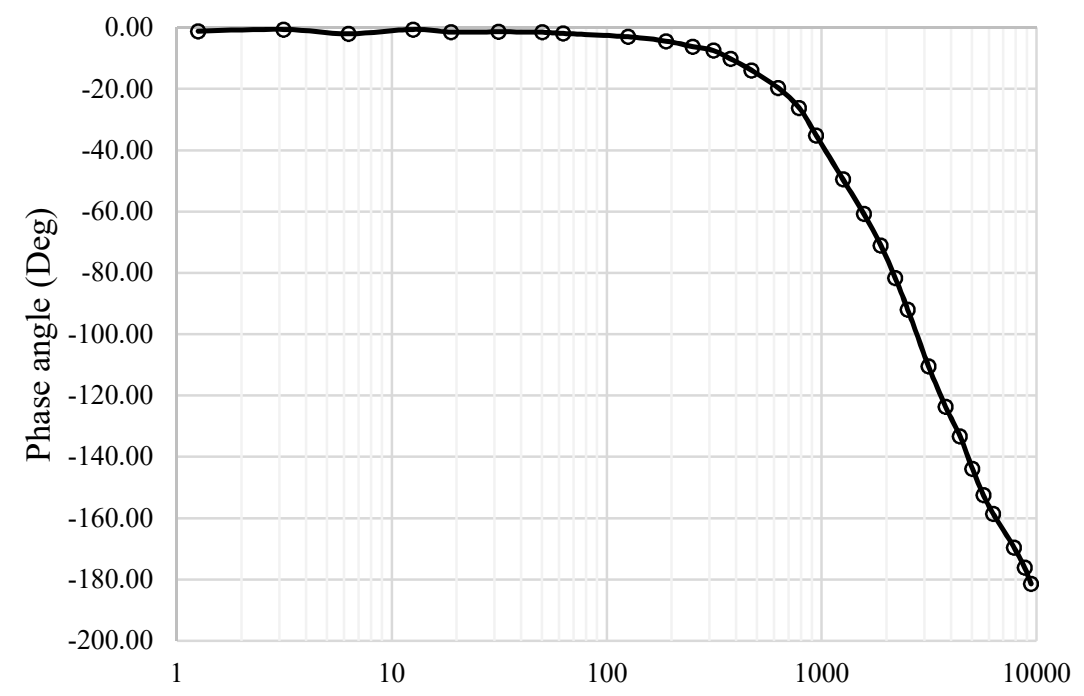

Frequency $(\mathrm{rad} / \mathrm{s})$

(b)

Fig. 2.17 Frequency response of the actuator (a) Magnitude response (b) Phase angle response. 
In dynamic performance test, a sinusoidal voltage varying from $20 \mathrm{~V} \sim 70 \mathrm{~V}$ is applied. The frequency response is measured using PSD and is shown in Fig. 2.17. The input driving voltage signals and the output voltage signals form the PSD are used to construct the bode plot. The measured resonant frequency is $380 \mathrm{~Hz}$. The discrepancy between the simulations $(557 \mathrm{~Hz})$ and testing results could be that the air damping due to squeeze film effect is not considered in the simulation. Also the middle beam slightly deforms during the actuation due to the large moment of inertial of the mirror plate attached to it. This deformation is counted in the measured frequency response.

\subsection{Summary}

A novel electrostatic repulsive-torque actuator which has two rows of interdigitating rotation fingers has been presented in this chapter. The actuator is able to generate large repulsive-torque and achieve an out-of-plane rotation with large angle and high reliability by avoiding the "pull-in" instability. The actuator requires only requires two thin layers and is suitable for surface micromachining. The mathematical model has been established to verify the actuator and evaluate its performance. The model is a hybrid approach based on 2D electric field numerical simulation and analytical equations. The modeling methodology is concluded into four steps and may serve as a reference approach to develop models for other electrostatic actuators with similar structures. The developed model provides a way to fast predict the static performance (rotation angle under DC voltage) of the actuator and is able to give the solutions for any given driving voltage in seconds. While it will take hours or days to get a solution if using numerical simulation. Even so when the driving voltage is relatively high $(\geq 100 \mathrm{~V})$ the coupled solver of the simulation software cannot converge and provides an inaccurate solution. Using the developed model, the actuator is able to achieve about $10.6^{\circ}$ rotation angle. Also the rotation angle and repulsive torques generated by the TRF actuator is compared with the previously developed ORF actuator which is proved to have a nearly $100 \%$ improvement.

The dynamic performance of the actuator is simulated using simulation software and the effect

of the upper and lower moving part on the resonant frequency of the actuator has been analyzed which provides a good guidance in designing similar actuator with frequency response restrictions.

Prototypes have been fabricated and tested. The measured mechanical rotation angle is $11.5^{\circ}$ for a driving voltage of $150 \mathrm{~V}$. The resonant frequency is measured to be $380 \mathrm{~Hz}$ using optical 
methods. The model of the actuator shows a good match with the experimental results even at large driving voltages. The presented actuator has the potential to be used in 2D micromirrors and is expected to have much larger scanning angle and faster dynamic response than the micromirror based on previously developed ORF actuator. 


\section{Chapter 3 Two-width-finger repulsive-torque electrostatic actuator}

This chapter is based on the following published paper:

Chao Fan and Siyuan He, "A microelectrostatic repulsive-torque rotation actuator with two-width fingers", Journal of Micromechanics and Microengineering, vol. 25, pp. 095006, 2015.

In this chapter, a micro electrostatic repulsive-torque rotation actuator with two-width fingers is presented which is able to achieve large out-of-plane rotation. It meets the first objective of the thesis. The actuator consists of finger shape electrodes and is made of two thin film layers, i.e., one movable layer and one fixed layer. There are two types of finger electrodes, i.e., the constant width and the two-width fingers. The two-width finger has a narrow lower segment and a wide top segment. The constant width finger has only the narrow lower segment. Each rotation finger has its corresponding aligned and unaligned fixed fingers. The electrostatic repulsive-torque is generated and acts on the rotation fingers to rotate them up and away from the substrate. As a result, the rotation is not limited by the gap between the movable and fixed layers and the "pullin" instability is avoided. Thus a large out-of-plane rotation and high operation stability can be achieved. The actuator is suitable for two-layer surface micromachining. The model of the actuator is developed. Prototypes are fabricated and tested. The experimental tests show that the actuator achieved a mechanical rotation of $7.65^{\circ}$ at a driving voltage of $150 \mathrm{~V}$. The settling time for a mechanical rotation of $5^{\circ}$ is $5.7 \mathrm{~ms}$.

\subsection{Design}

\subsubsection{Structure}

The actuator consists of constant-width rotation fingers, two-width rotation fingers, aligned fixed fingers and unaligned fixed fingers, as shown in Fig. 3.1. Each of the two-width finger consists of a lower narrow segment and a top wide segment. The aligned fixed fingers (constantwidth and two-width) are aligned with the corresponding rotation fingers. All rotation fingers are connected to a common beam. A micro mirror plate is connected to the middle rotation finger for measuring the rotation angle. Two serpentine springs are connected to the common beam. There 
are 10 constant-width rotation fingers and 7 two-width rotation fingers. Rotation fingers and aligned fixed fingers are subject to the same electric potential while the unaligned fingers are subject to a different electric potential. The repulsive-torque is generated on rotation fingers to rotate them up and away from the fixed fingers without suffering from the "pull-in" instability.

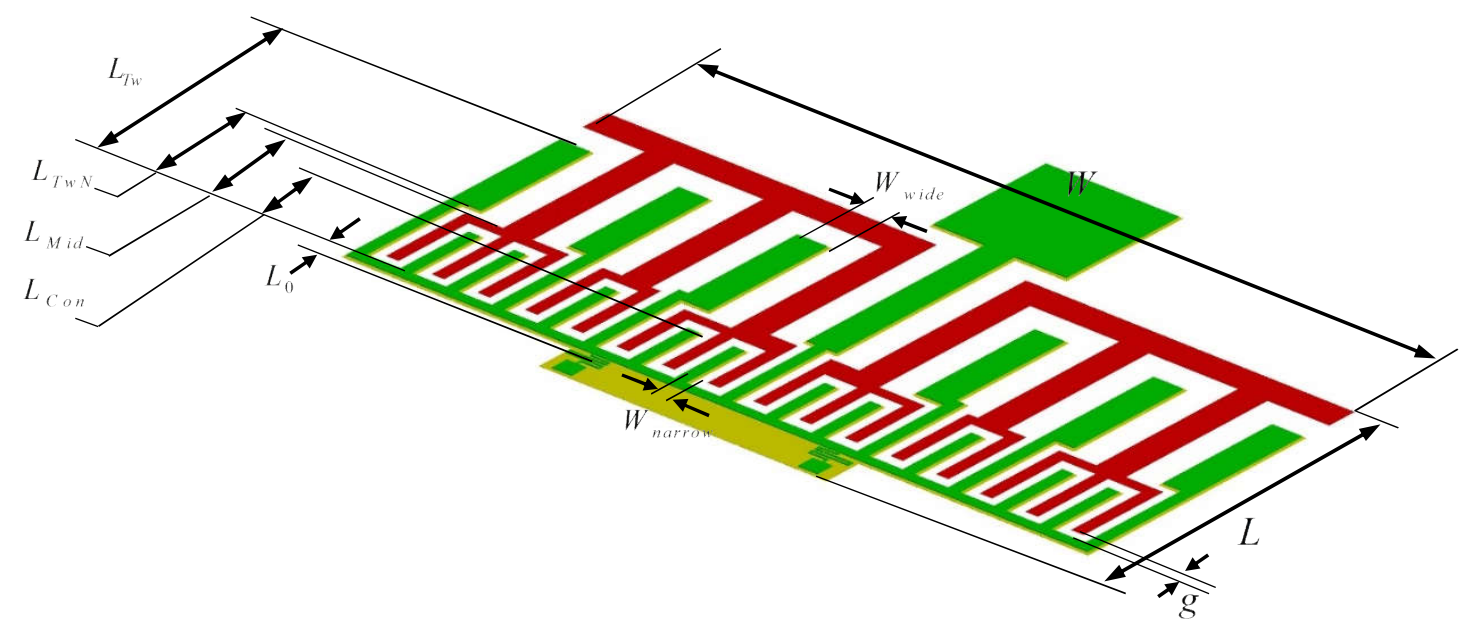

(a)

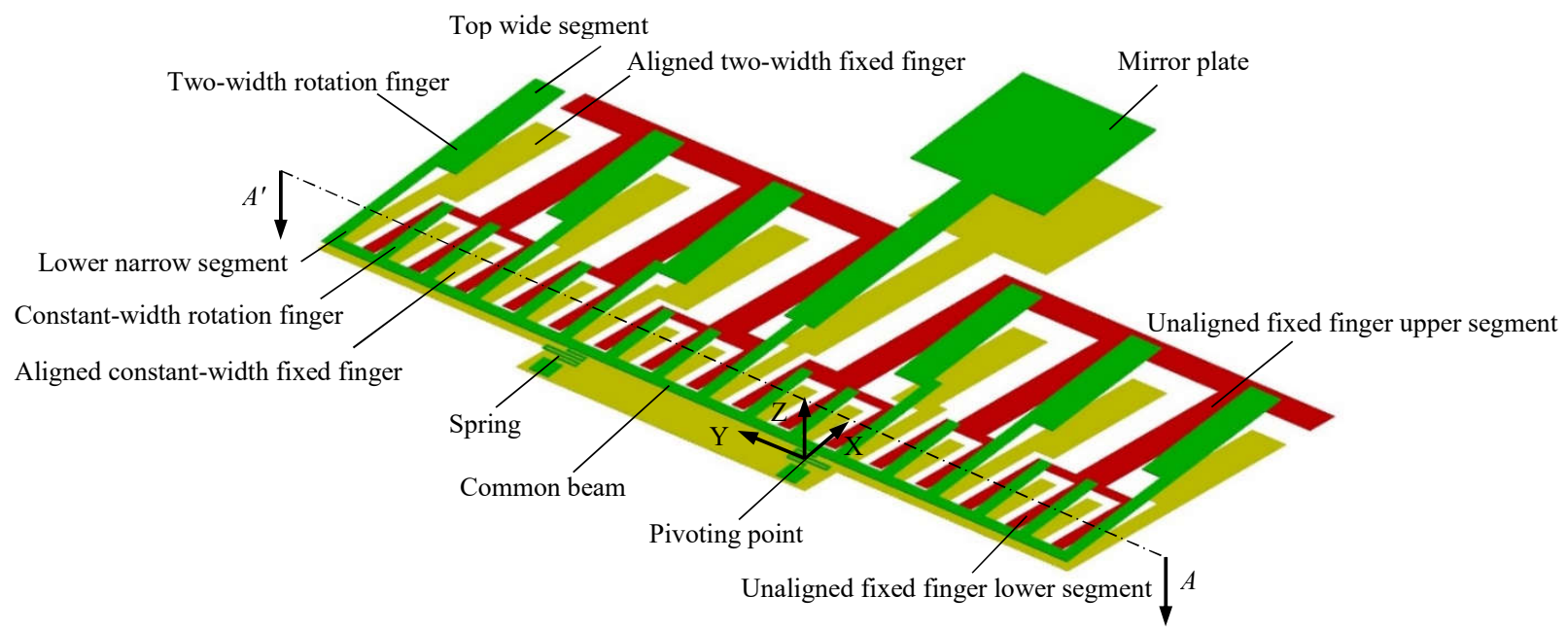

(b)

Fig. 3.1 Two-width finger actuator. (a) The actuator is at initial position. (b) The actuator is in operation.

The central four constant-width rotation fingers are of $25 \mu \mathrm{m}$ shorter than the other six constant-width rotation fingers in order to use that $25 \mu \mathrm{m}$ as the horizontal electrode connecting the lower and upper segments of the two-width fingers beside the middle beam. As a result, the top wide segment does not need to sacrifice its length to leave space for the horizontal connection 
electrode in order to have the same length as that of the top wide segment of the rest two-width rotation fingers.

\subsubsection{Working principle}

The repulsive-force can be generated on all rotation fingers according to the analysis in [97]. It has been analyzed in [105] that the repulsive-force decreases along the finger length when the actuator rotates with decreasing rate inversely related to the finger width. Detailed explanation can be found in [97]. In a repulsive-force rotation actuator with one-width finger as those previously reported in [97], [105], [107] the repulsive-force decreases along the finger length and approaches to zero at the top part of each rotation finger when the rotation angle is large. Thus the top part of the finger has no contribution to the total torque. If the top part becomes wider than the bottom part, i.e., the finger has two widths along its length, the repulsive-force on the top part does not decrease to zero because of the slower force decreasing rate due to its wider finger width. As a result, a higher total repulsive-torque and then rotation angle can be obtained. The quantitative modeling will be presented in the next section. The actuator includes the lower and top parts. Each part has the same elementary cell as that in the one-width repulsive-force actuators previously reported [97], [105], [107]. The "repulsive-force" exerted on the rotation fingers is the net force after summing the forces on both the bottom and top surfaces. Detailed explanation on how the parameters of fingers and distance between fingers affect the actuator performance can be found in the references [97], [105], [107].

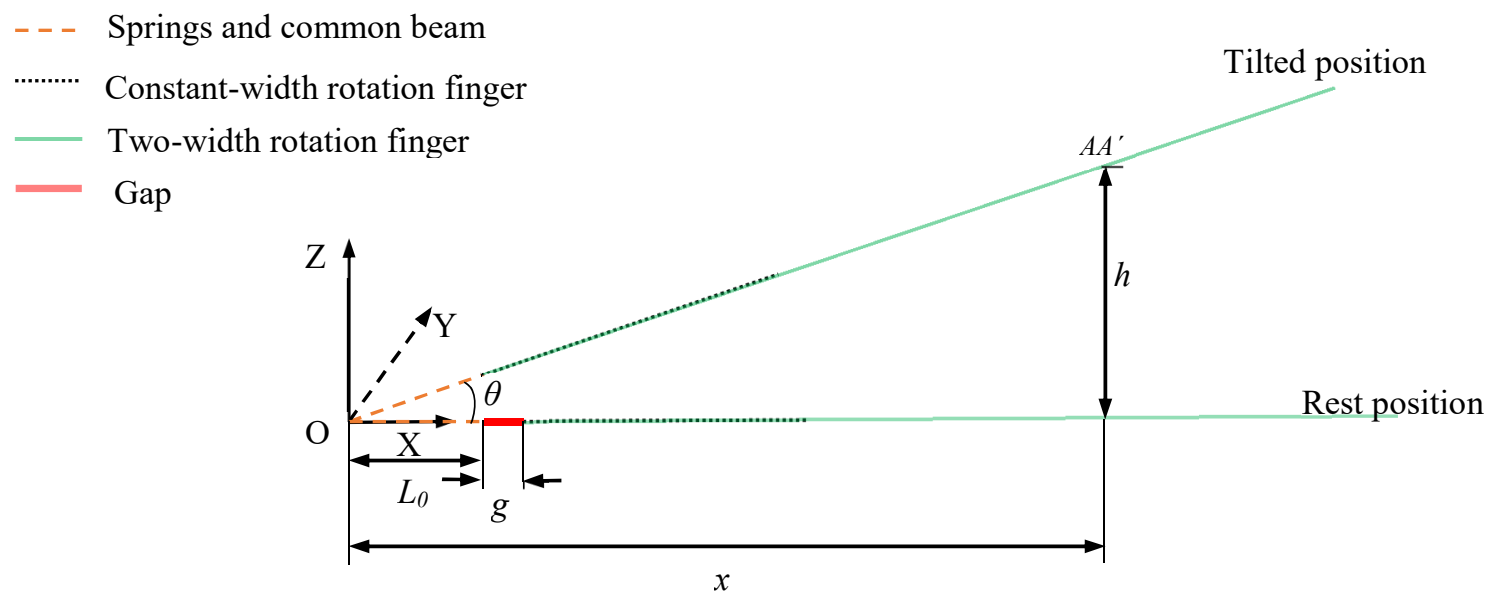

Fig. 3.2 Side view of two-width finger actuator. 


\subsection{Modeling}

A coordinate system XYZ is built with the origin located at the pivoting points (see Fig. 3.1(b)) as shown in Fig. 3.2. The pivoting points are selected to be at the center of the spring. $\mathrm{X}$-axis is along the finger length direction. Y-axis is along the common beam length direction. Actuator parameters are listed in Table. 3.1.

The following explain variables (see Fig. 3.1(a) and Fig. 3.2).

$L$ and $W$ : Overall length and width of the actuator.

$W_{\text {wide }}$ and $W_{\text {narrow: }}$ Finger widths of wide and narrow segments of two-width rotation finger. The finger width of constant-width finger is the same as that of the lower narrow segment of two-width rotation finger.

$L_{0}$ : Distance from the pivoting point, i.e., origin $O$, to the upper edge of the common beam where it connects the rotation fingers.

g: The gap between the upper edge of the common beam and the lower edge of unaligned fixed fingers.

$L_{C o n}$ : The length of the constant-width rotation finger, which is defined from its tip to the upper edge of the common beam. $L_{C o n}$ uses the finger length of four central constant-width fingers for all ten constant-width fingers. The inaccuracy caused decreases from the low to high driving voltage, e.g., the difference between the calculation using the long constant-width fingers' length and the calculation using the short constant-width fingers' length is about $10 \% \sim 1 \%$ when the driving voltage varies from $0 \mathrm{~V}$ to $150 \mathrm{~V}$. The real value should be higher than the calculation using short finger length and lower than the calculation using long finger length. Such that the inaccuracy is estimated to be less than $10 \% \sim 1 \%$ when the driving voltage varying from $0 \mathrm{~V}$ to $150 \mathrm{~V}$.

$L_{\text {Mid }}$ : The distance from the upper boundary of the middle part (shown in Fig. 3.3) to the upper edge of the common beam.

$L_{T w N}$ : The length of the narrow segment of the two-width rotation finger, which is defined from its connecting point with the common beam to the intersection of the wide and narrow segments. 
$L_{T w}$ : The length of the two-width rotation finger which is defined from its tip to the upper edge of the common beam.

$\theta$ : The rotation angle of rotation fingers.

$x$ : X-axis coordinate of a cross section (such as the one cut by the line $A A^{\prime}$ in Fig. 3.1(b) and Fig. 3.7).

$h$ : The vertical distance from the tilted rotation finger to its initial position.

Table 3.1 Parameters of two-width finger actuator (unit: micrometer)

\begin{tabular}{c|c|c|c|c|c|c|c|c|c}
\hline \hline$L$ & $W$ & $W_{\text {wide }}$ & $W_{\text {narrow }}$ & $L_{0}$ & $\mathrm{~g}$ & $L_{\text {Con }}$ & $L_{\text {Mid }}$ & $L_{T w N}$ & $L_{T w}$ \\
\hline 796 & 1685 & 67.5 & 25 & 46 & 30 & 157 & 237 & 257 & 548 \\
\hline \hline
\end{tabular}

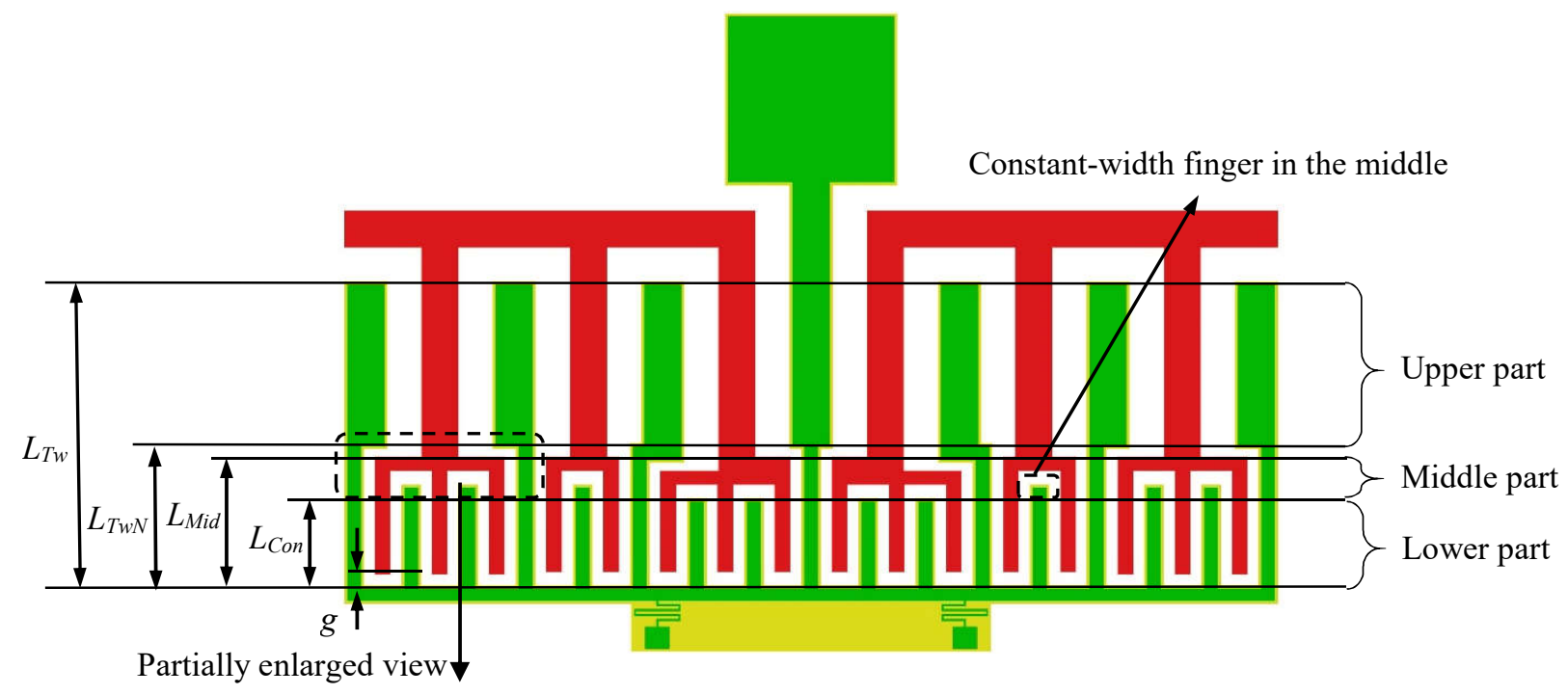

(a)

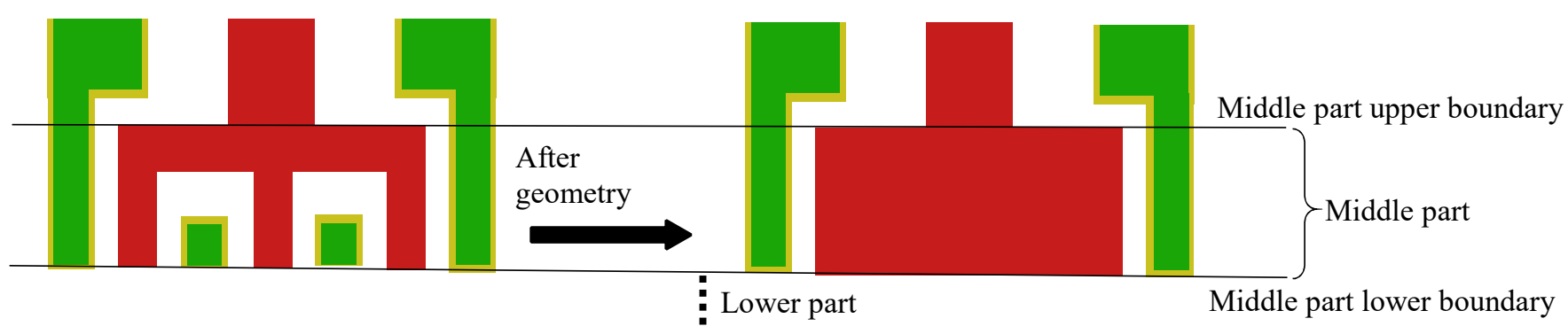

(b)

Fig. 3.3 (a) Top View of the actuator. (b) Enlarged view of part of the middle part. 
The electrostatic torque exerted on rotation fingers includes three parts, i.e., torques on the lower, middle and upper parts as shown in Fig. 3.3. The lower part includes constant-width rotation fingers and the lower narrow segments of the two-width fingers. The upper part is composed of all top wide segments of the two-width fingers. The middle part includes the parts between the lower and upper parts of two-width rotation fingers. The small segments at the top of six longer constantwidth fingers in the middle part are neglected in the modeling as shown in Fig. 3.3 (a). In the middle part the approximation as shown in Fig. 3.3 (b) is made to calculate the electrostatic forces exerted on the narrow segments of two-width rotation fingers. The approximation is based on the analysis in [97], which explains the force on the rotation finger from the adjacent unaligned fixed fingers is mainly from the edge of the unaligned fixed finger. The electrostatic torques exerted on the lower, middle and upper parts are denoted as $T_{L}, T_{M}$, and $T_{U}$ respectively.

In static operation, the electrostatic repulsive-torque acting on the rotation fingers is balanced by the mechanical restoring torque as expressed in Eq. (3.1).

$$
T_{L}+T_{M}+T_{U}=K_{\text {rotation }} \cdot \theta
$$

Where $K_{\text {rotation }}$ is the rotational stiffness of the actuator which can be obtained from simulation. In the stiffness simulation based on FEM, a mechanical upward force is applied to the tips of twowidth rotation fingers as shown in Fig. 3.4. The simulated stiffness is the rotation stiffness about Y-axis (see Fig. 3.1(b). The long common beam slightly bends in operation, i.e., the vertical displacement of the middle part is not the same as its two ends. The stiffness simulated using the current method does not only include the stiffness of the springs and common beam structure, but also the stiffness of each two-width rotation finger even its stiffness is much higher (a few times) than that of the springs and common beam structure. The Coventorware software is used for the simulation and the mesh size convergence has been studied. The structure stiffness versus the number of elements is plotted in Fig. 3.5. $N$ is the number of element of the coarsest mesh which is 3364. It can be seen that the simulation result tend to converge when the mesh size is set to about 12 times of $N$. The corresponding stiffness obtained is about $3.8 \times 10^{-9} \mathrm{Nm} / \mathrm{rad}$. 


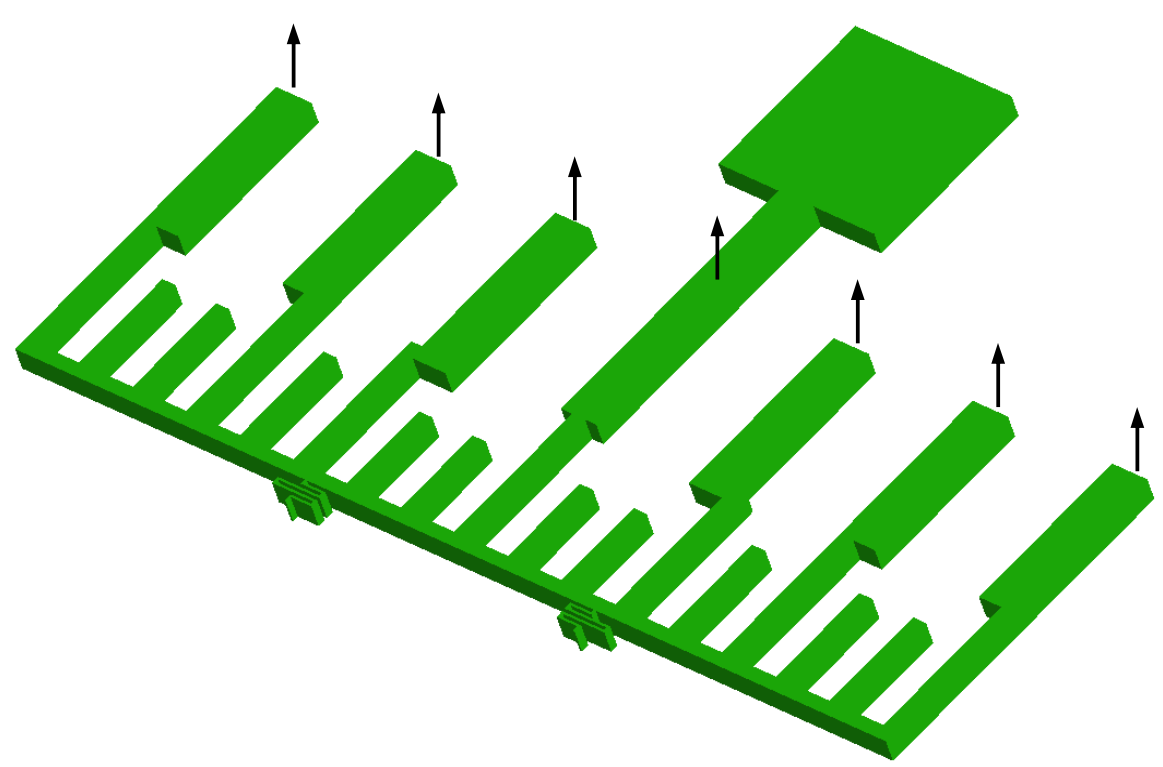

Fig. 3.4 Simulation of rotation stiffness about Y-axis (see Fig. 3.1(b)).

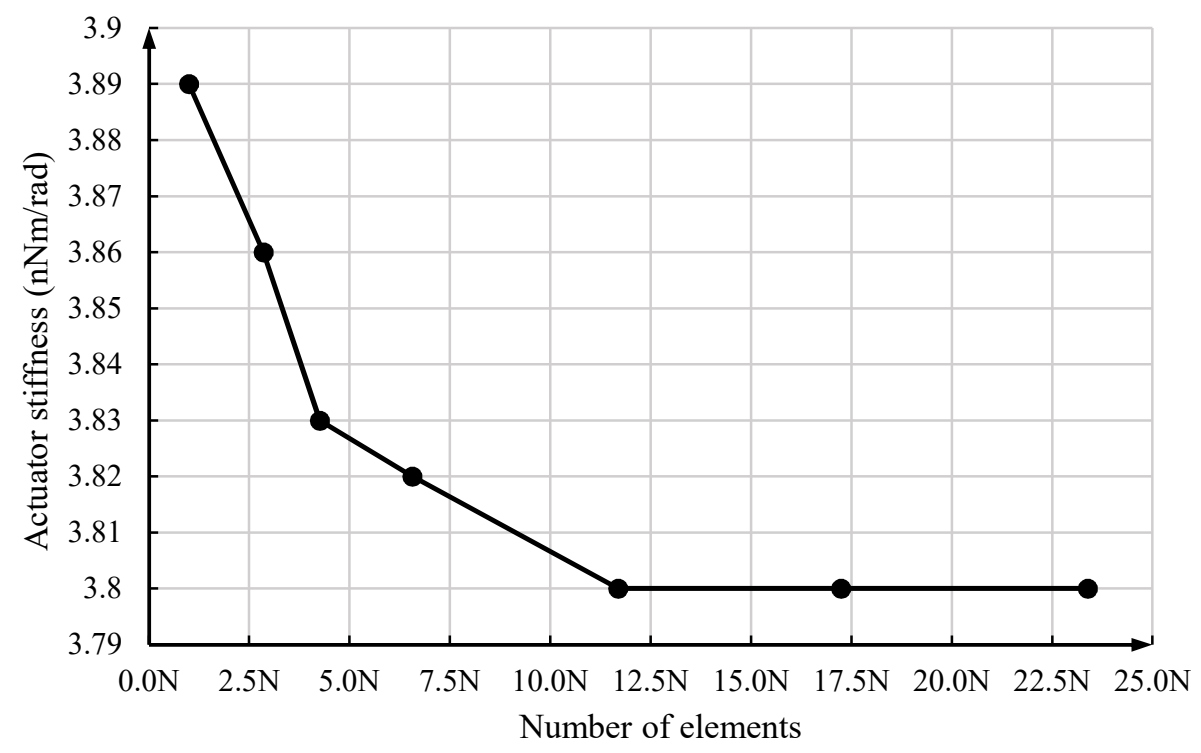

Fig. 3.5 Mesh size convergence study.

The electrostatic forces on each cross section of the lower, middle and upper parts are obtained using 2D electric field simulations. The results are fitted with polynomials. The total electrostatic torque can be obtained by integrating the cross section force multiplied by the distance from the cross section to the pivoting point as follows.

$$
T_{L}(V, \theta)=V^{2} \cdot \int_{L_{0}+g}^{L_{0}+L_{C o n}} f_{L}(x, \theta) x d x
$$




$$
\begin{gathered}
T_{M}(V, \theta)=V^{2} \cdot \int_{L_{0}+L_{C o n}}^{L_{0}+L_{M i d}} f_{M}(x, \theta) x d x \\
T_{U}(V, \theta)=V^{2} \cdot \int_{L_{0}+L_{T w N}}^{L_{0}+L_{T w}} f_{U}(x, \theta) x d x
\end{gathered}
$$

Where $f_{L}, f_{M}$ and $f_{U}$ are the cross section electrostatic forces on the lower, middle and upper parts respectively with unit driving voltage.

The cross sections are shown in Fig. 3.6 (a), (b) and (c), which are not to scale. Only a few fingers are shown in the figure and the periodical structure repeats in both directions. Only half of the middle part cross section is plotted and the other half is symmetrical. There are total 17 rotation fingers in lower part and 7 rotation fingers in middle and upper parts. The rotation fingers are 0.75 $\mu \mathrm{m}$ away from the fixed fingers which is the height of the dimples in PolyMUMPs [112]. Electro software [142] is used to perform 2D electric field simulations. $h$ is the vertical displacement of a point on rotation fingers to their initial positions when the actuator is at rest. The ranges of $h$ used for the simulations are different for the lower, middle and upper parts. In order to determine the range of h, $\theta$ (the actuator rotation angle) range needs to be estimated. $\theta$ range needs to be estimated as large as possible to make $\mathrm{h}$ range wide enough for simulation. From the experimental test, $\theta$ is less than $9^{\circ}$. Thus the range of $\theta$ is taken as $0^{\circ} \sim 9^{\circ}$. Ranges of $h$ can then be determined using Eq. (3.5) for each part, e.g., $h \in[0,33 u m]$ for the lower part, $h \in[0,48 u m]$ for the middle part and $h \in[0,95 \mathrm{um}]$ for the upper part. The range of $x$ for the lower, middle and upper parts can be calculated using the following equation.

$$
h=\tan \theta \cdot x
$$

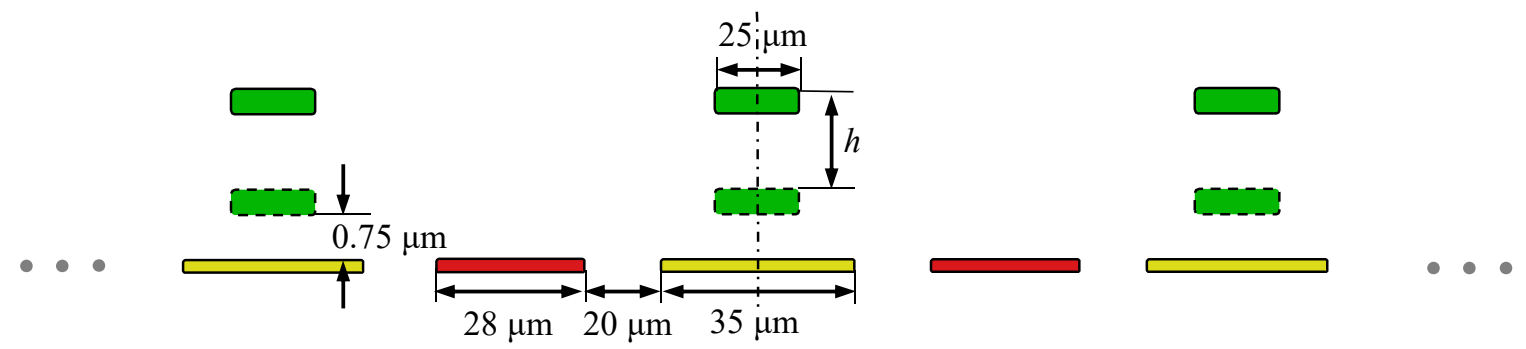

Constant-width finger and narrow segments of two-width rotation fingers at initial position

$\square$ Constant-width finger and narrow segments of two-width rotation fingers in operation

$\square$ Aligned fixed finger

$\square$ Unaligned fixed finger (lower segment) 


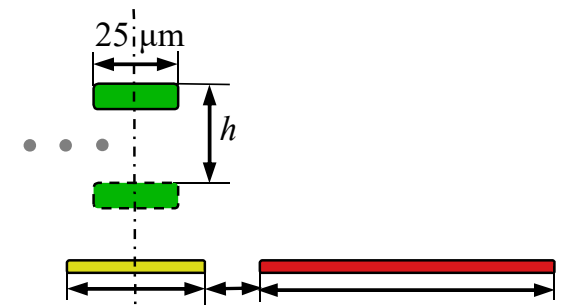

$35 \mu \mathrm{m} 20 \mu \mathrm{m}$

$233 \mu \mathrm{m}$

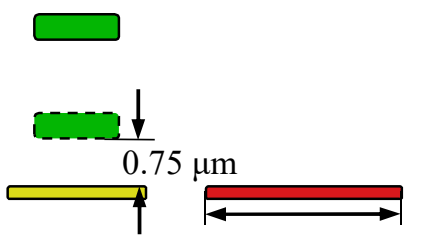

$131 \mu \mathrm{m}$

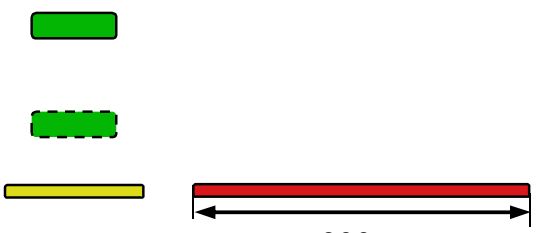

$233 \mu \mathrm{m}$

Ez-ב Two-width rotation finger (lower segment) at initial position

$\square$ Two-width rotation finger (lower segment) in operation

$\square$ Aligned two-width fixed finger (lower segment)

$\square$ Unaligned fixed finger (lower segment)

(b)

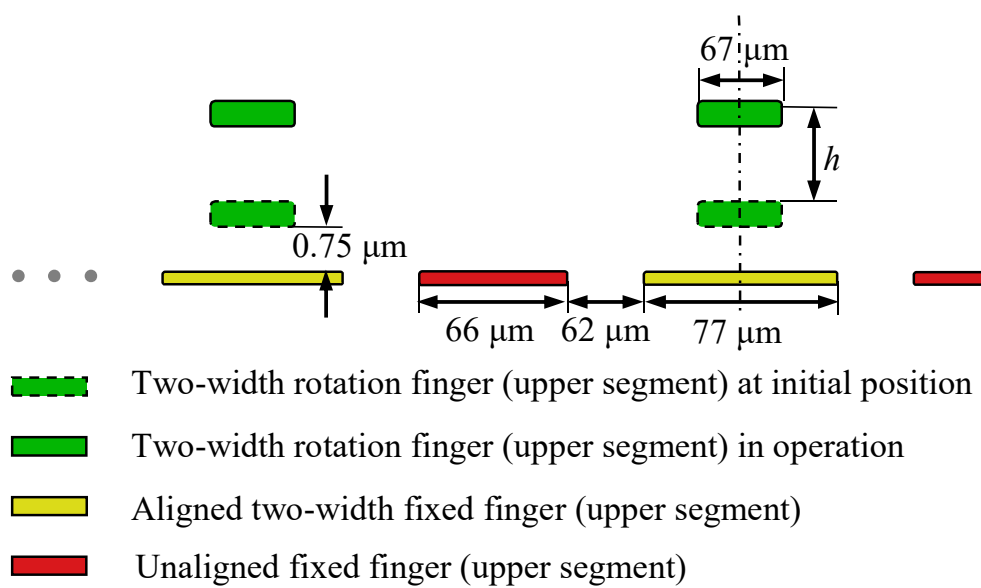

(c)

Fig. 3.6 Schematic of cross sections. (a) Part of the lower part (b) Half of the middle part (c) Part of the upper part.

Based on the simulation results the curve fitting method using polynomial function is used to obtain $f_{L}, f_{M}$ and $f_{U}$. The fitting polynomials are expressed in Eqs. (3.6) (3.8). R-square is used as the fitting criteria. The orders of polynomials for $f_{L}, f_{M}$ and $f_{U}$ are selected so that the R-square for each fitting is larger than 0.99. The fitting curves and simulation data are shown in Fig. 3.7.

$$
f_{L}(h)=P_{1 L} h^{5}+P_{2 L} h^{4}+P_{3 L} h^{3}+P_{4 L} h^{2}+P_{5 L} h+P_{6 L}
$$

Where $P_{1 L}=3.487 \mathrm{e}-13, P_{2 L}=-3.453 \mathrm{e}-11, P_{3 L}=1.28 \mathrm{e}-09, P_{4 L}=-2.034 \mathrm{e}-8, P_{5 L}=6.091 \mathrm{e}-08, \mathrm{P}_{6 \mathrm{~L}}=$ $1.414 \mathrm{e}-06$. The units of all the coefficients are omitted, and they enable $f_{L}$ to have a unit of N/m/ $/ \mathrm{V}^{2}$.

$$
f_{M}(h)=P_{1 M} h^{6}+P_{2 M} h^{5}+P_{3 M} h^{4}+P_{4 M} h^{3}+P_{5 M} h^{2}+P_{6 M} h+P_{7 M}
$$


Where $P_{1 M}=-1.874 \mathrm{e}-15, P_{2 M}=3.085 \mathrm{e}-13, P_{3 M}=-2.004 \mathrm{e}-11, P_{4 M}=6.492 \mathrm{e}-10, P_{5 M}=-1.051 \mathrm{e}-8$, $P_{6 M}=4.784 \mathrm{e}-08, P_{7 M}=8.938 \mathrm{e}-07$. The units of all the coefficients are omitted, and they enable $f_{M}$ to have a unit of $\mathrm{N} / \mathrm{m} / \mathrm{V}^{2}$.

$$
f_{U}(h)=P_{1 U} h^{7}+P_{2 U} h^{6}+P_{3 U} h^{5}+P_{4 U} h^{4}+P_{5 U} h^{3}+P_{6 U} h^{2}+P_{7 U} h+P_{8 U}
$$

Where $P_{1 U}=3.296 \mathrm{e}-19, P_{2 U}=-1.197 \mathrm{e}-16, P_{3 U}=1.755 \mathrm{e}-14, P_{4 U}=-1.328 \mathrm{e}-12, P_{5 U}=5.465 \mathrm{e}-11$, $P_{6 U}=-1.131 \mathrm{e}-09, P_{7 U}=4.127 \mathrm{e}-09, P_{8 U}=2.678 \mathrm{e}-7$. The units of all the coefficients are omitted, and they enable $f_{U}$ to have a unit of $\mathrm{N} / \mathrm{m} / \mathrm{V}^{2}$.

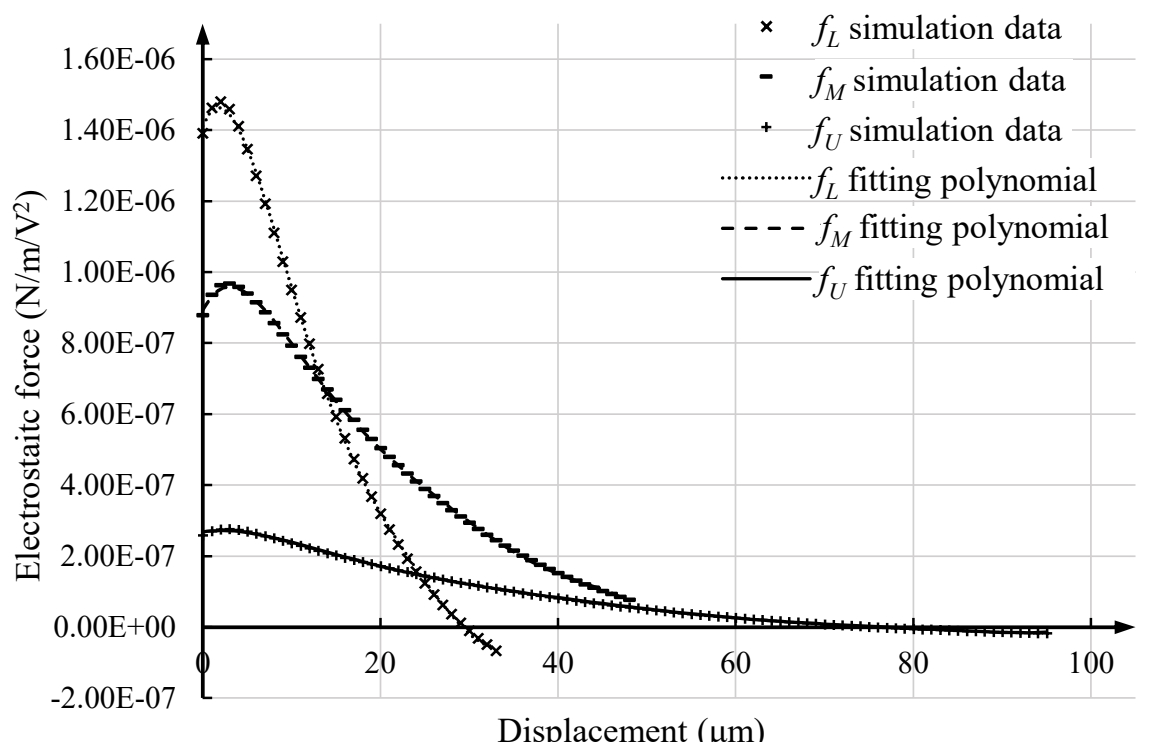

Fig. 3.7 Fitting curves and simulation data.

After substituting Eq. (3.5) into Eqs. (3.6) (3.8), $f_{L}, f_{M}$ and $f_{U}$ are functions of $\theta$. Substituting the resultant equations into Eq. (3.1), the rotation angle of the actuator can be solved for any given driving voltage. The corresponding electrostatic torque on the rotation fingers can be obtained from Eqs. (3.2) (3.4).

\subsection{Performance evaluation}

\subsubsection{Rotation angle estimation}

The rotation angle of the actuator calculated according to the model is plotted in Fig. 3.8 (a), which shows the two-width-finger (TWF) actuator developed in this chapter can generate a mechanical rotation of $6.74^{\circ}$ at $150 \mathrm{~V}$. The ORF actuator is designed using the optimization method in [105] with the same stiffness and actuator area as those of the actuator presented in this 
chapter. The percentage of error between the calculated rotation angles of the TWF actuator and the previously developed ORF actuator is shown in Fig. 3.8 (b). It can be concluded that over the entire applied voltage range of $0 \mathrm{~V}$ to $150 \mathrm{~V}$, the TWF actuator has larger rotation angles especially when the voltage is high $(>30 \mathrm{~V})$. The largest improvement happens in the voltage range $50 \mathrm{~V} \sim$ $60 \mathrm{~V}$ which is $22.6 \%$. At the voltage $150 \mathrm{~V}$ which is the maximum voltage used for tests, the improvement is about $19.2 \%$.

\subsubsection{Dynamic response estimation}

Modal analysis is performed using Coventorware software [143]. The first, second and third mode are $392 \mathrm{~Hz}, 1246 \mathrm{~Hz}$, and $1479 \mathrm{~Hz}$ respectively. The first mode deformation is shown in Fig. 3.9. The desired operation of the actuator is the first mode which rotate with respect to the two springs at the bottom. The resonant frequency found for the first mode is without considering the effect of air damping. It can be observed from Fig. 3.9 that the middle beam has a slightly different deflection with the other fingers. It is because that there is a narrow segment in the middle beam and a relative large mirror plate is attached to the end.

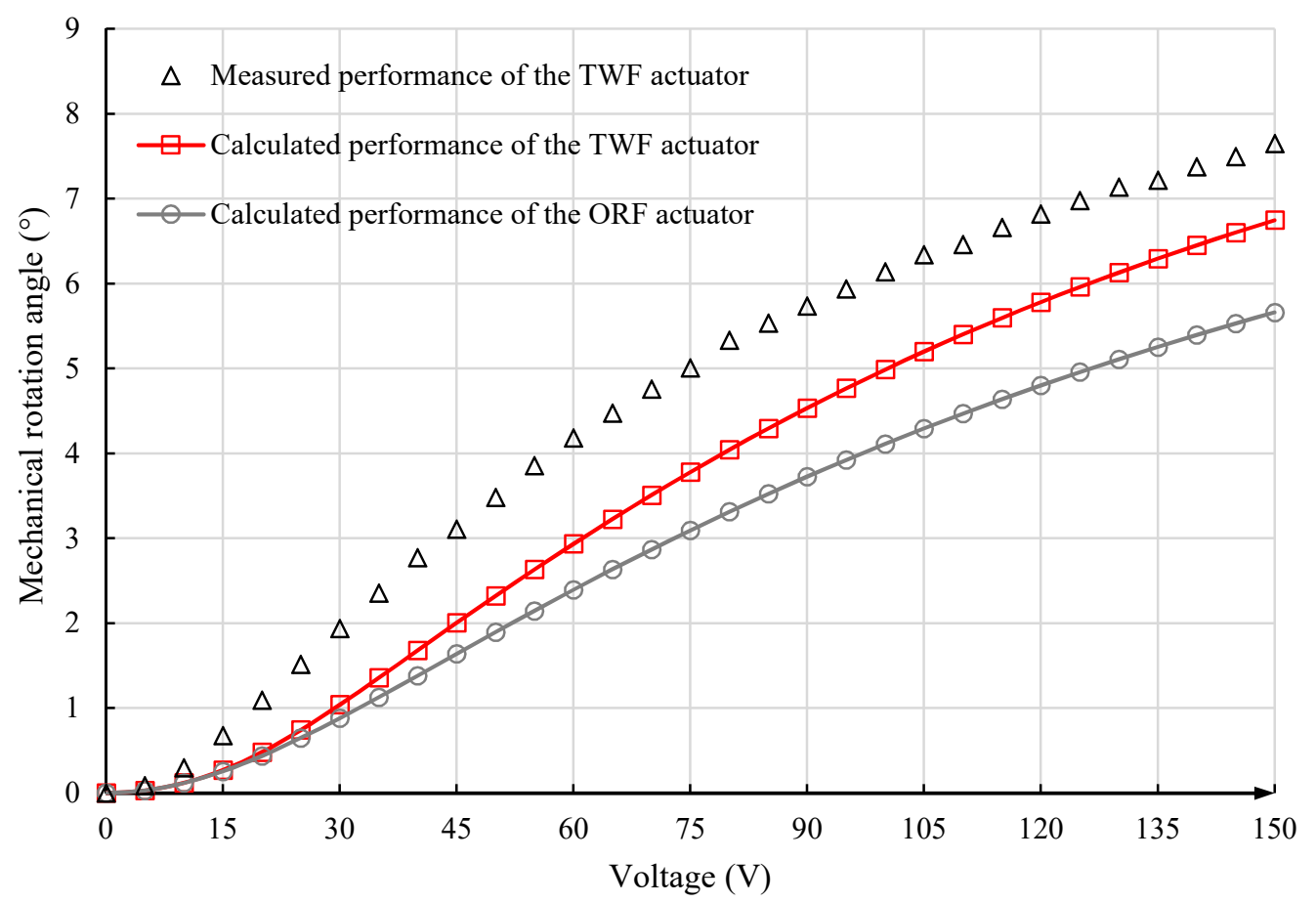

(a) 


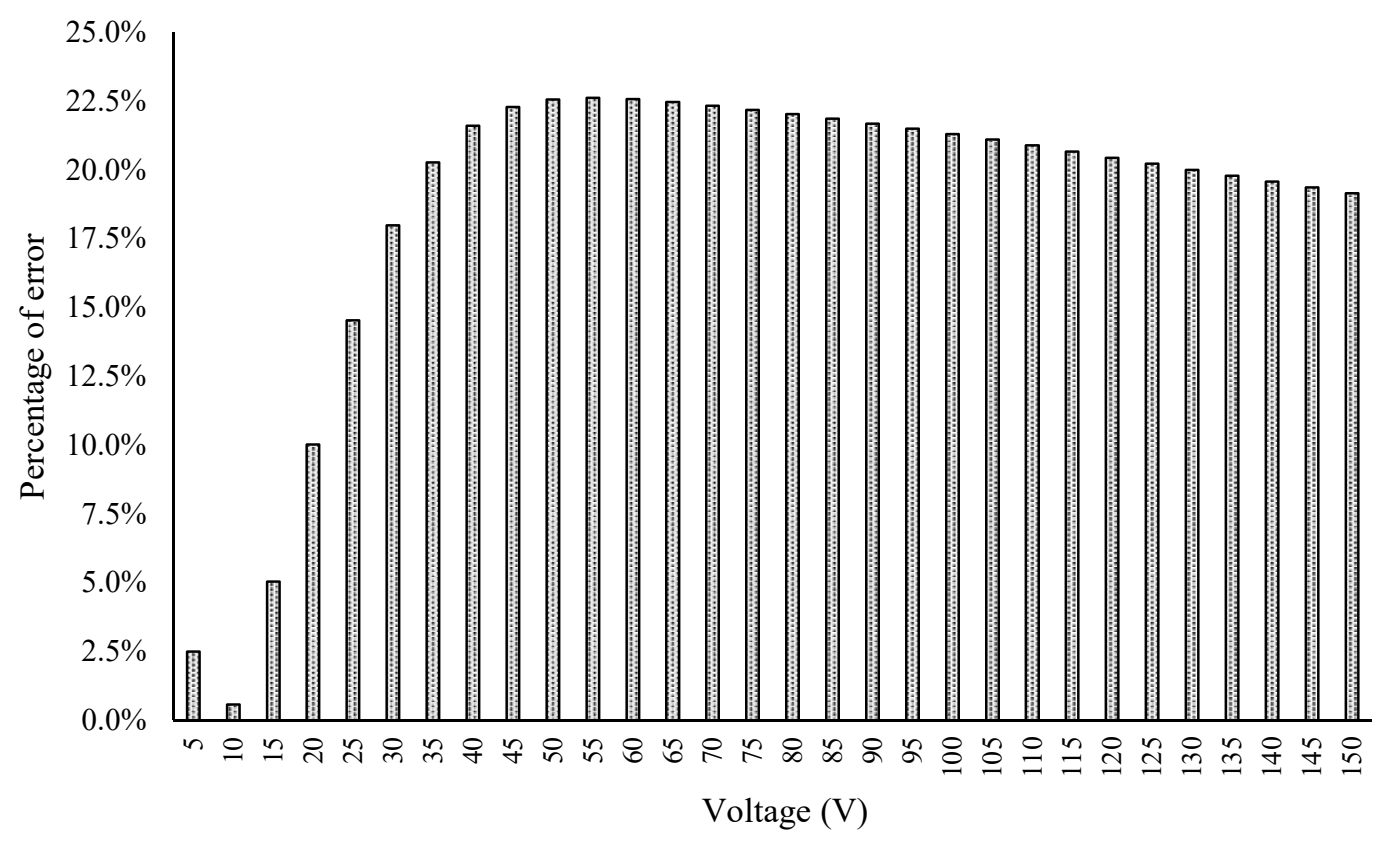

(b)

Fig. 3.8 (a) Modeling and experimental results (b) Percentage of the improvement of the TWF actuator over the previously developed ORF actuator.

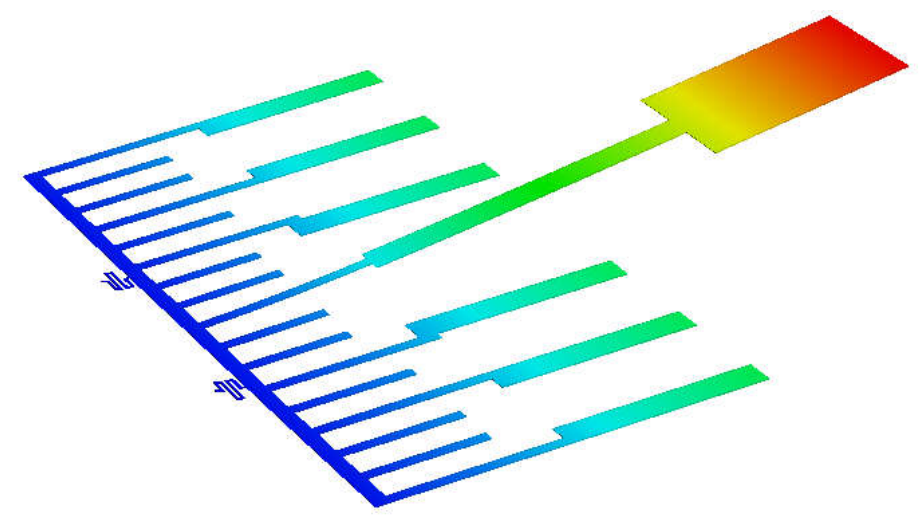

Fig. 3.9 First mode deformation of the two-width finger actuator. 


\subsubsection{Verify the estimation of $L_{c o n}$}

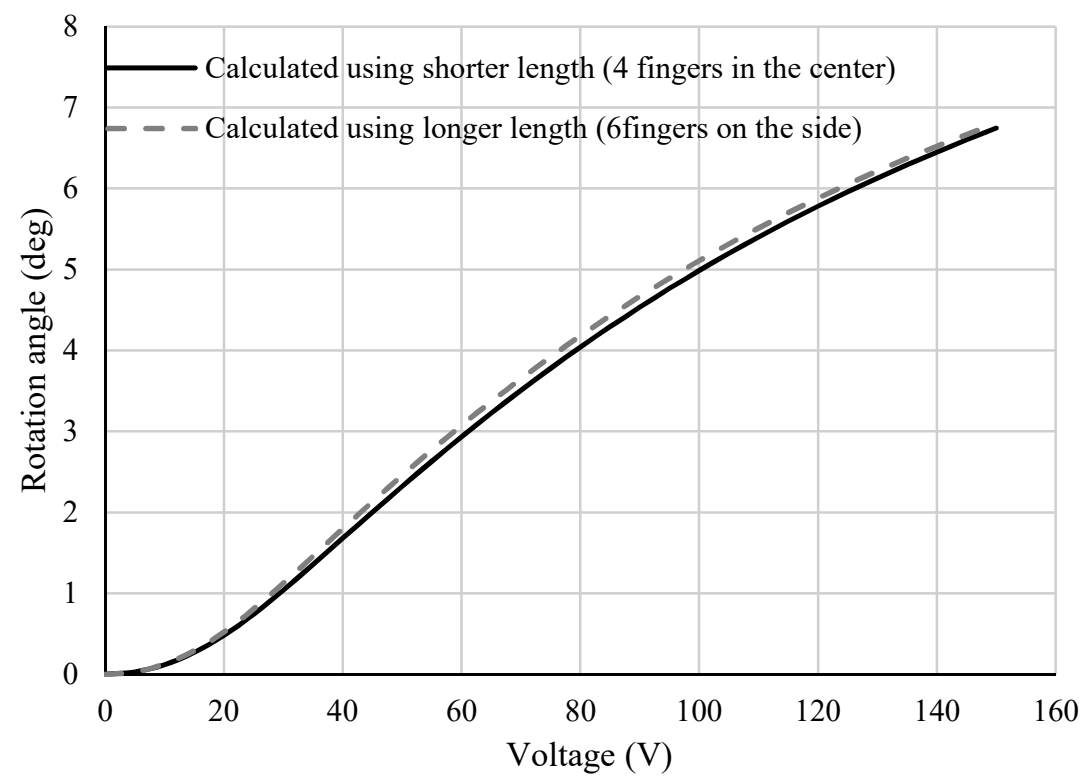

Fig. 3.10 Calculated rotation angles when $L_{C o n}$ is set to the length of the 4 fingers in the center and 6 finger on the side separately.

It was mentioned in section 3.2 that due to the design requirement, the length of the 10 constant-width fingers are slightly different, i.e., the central 4 constant-width fingers are $25 \mu \mathrm{m}$ shorter than the other 6 constant-width fingers. This is simplified in the modeling process by assuming all 10 fingers have the same length and $L_{c o n}$ is equal to the length of 4 central fingers. The error caused by this assumption can be estimated using the developed model. This can be done by using different values of $L_{c o n}$ in the model and calculate the resultant rotation angles. As a result, the length of both the central 4 fingers and the other 6 fingers are used in the model separately to obtain the corresponding rotation angles for voltage changing from 0 to $150 \mathrm{~V}$. The calculated results are plotted in Fig. 3.10.

From Fig. 3.10 it can be concluded that using the finger length of the 6 longer fingers can achieve larger rotation angles than that achieved using the shorter finger length. But the difference is very small. The maximum difference between the two curves is about $10 \%$ which happens when the driving voltage is smaller than $20 \mathrm{~V}$. The calculated results are two extremes conditions and the real rotation angles with different length of constant-width finger should lie between the two. As a result, it can be concluded that using the length of the 4 central fingers to represent the finger length of all constant-width fingers leads to $\sim 10 \%$ error at low voltage and $5 \%$ at $60 \mathrm{~V}$ and $<3 \%$ 
at $100 \mathrm{~V}$. Normally the driving voltage is high $(>100 \mathrm{~V})$ for most of applications using this actuator, that leads to negligible errors due to the approximation of $L_{C o n}$. The approximation of $L_{C o n}$ can significantly simplify the complexity of modeling

\subsection{Prototype and performance testing}

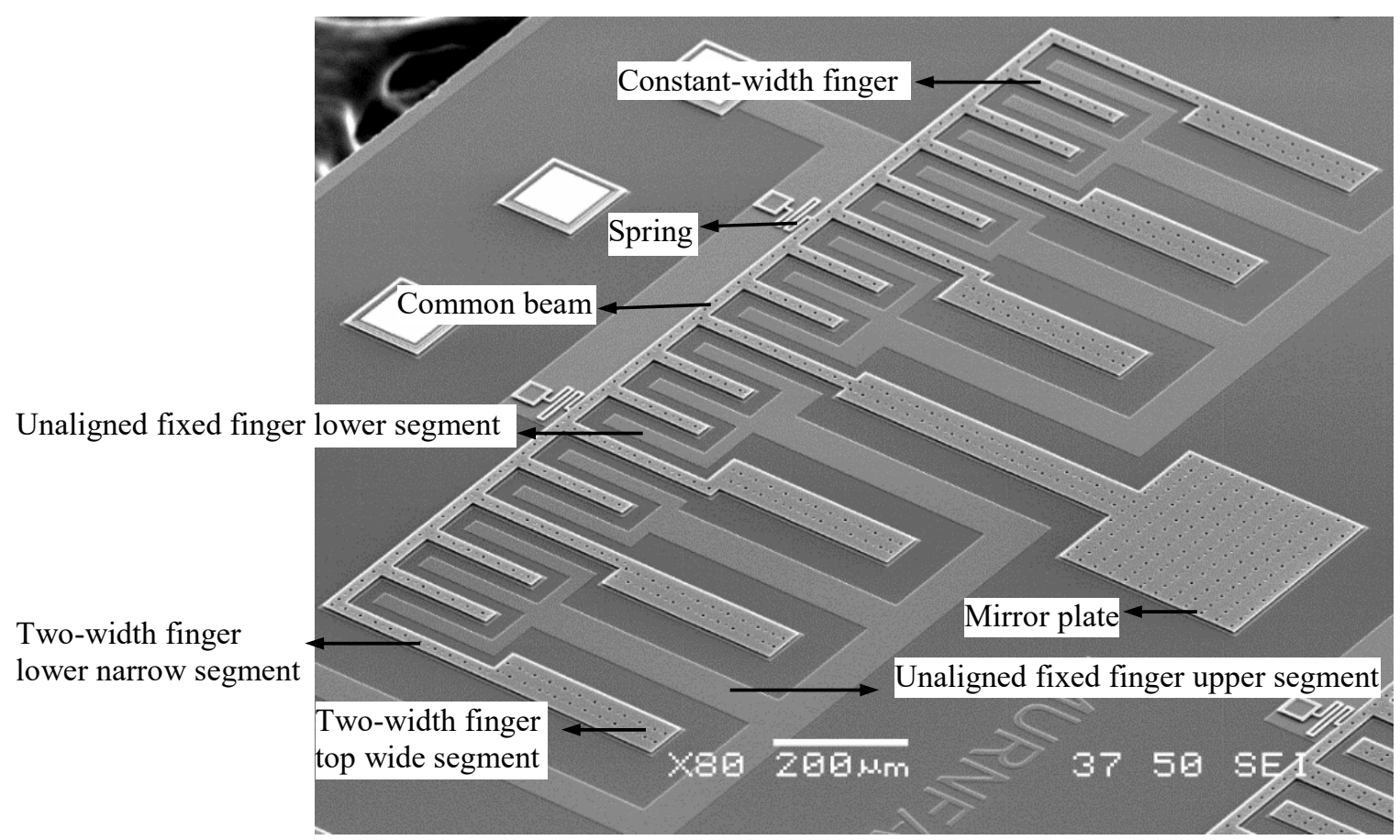

(a)

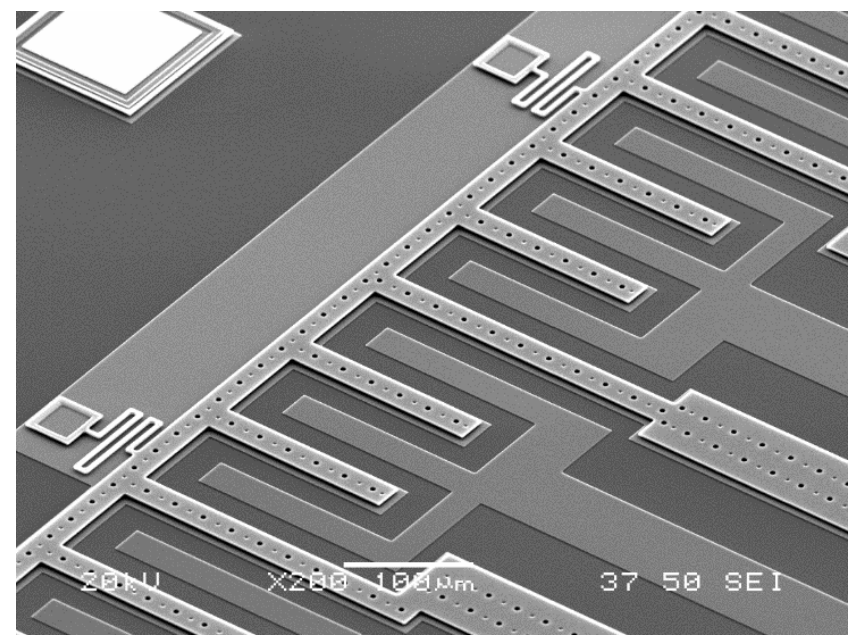

(b)

Fig. 3.11 SEM image of the TWF actuator (1) Overall view (2) Zoomed in view.

The actuator is suitable for two-layer surface micromachining. The prototype is fabricated using commercial surface micromachining process, i.e., PolyMUMPs [112]. In order to comply 
with the fabrication rule and avoid undesired lateral force [97], the aligned fixed finger is $5 \mu \mathrm{m}$ larger on each side than the corresponding rotation finger. Only Poly0 and Poly1 layers in PolyMUMPs are used. The SEM image of the prototype is shown in Fig. 3.11.

\subsubsection{Static performance}

The rotation angles of the actuator under various DC driving voltages are tested using the setup shown in Fig. 3.12. A red laser is used to focus the laser beam on the micro mirror plate of the actuator. Linear stages are used to adjust the micro mirror plate to be perpendicular to the optical bench. A grid paper is used as the display screen which is perpendicular to the reflected laser beam to record the displacement of the laser spot under various driving voltages. The rotation angle is calculated by knowing the laser spot displacement on the display screen and the distance between the screen and the actuator. A function generator is used to generate the testing signal which is amplified by a voltage amplifier to drive the actuator.

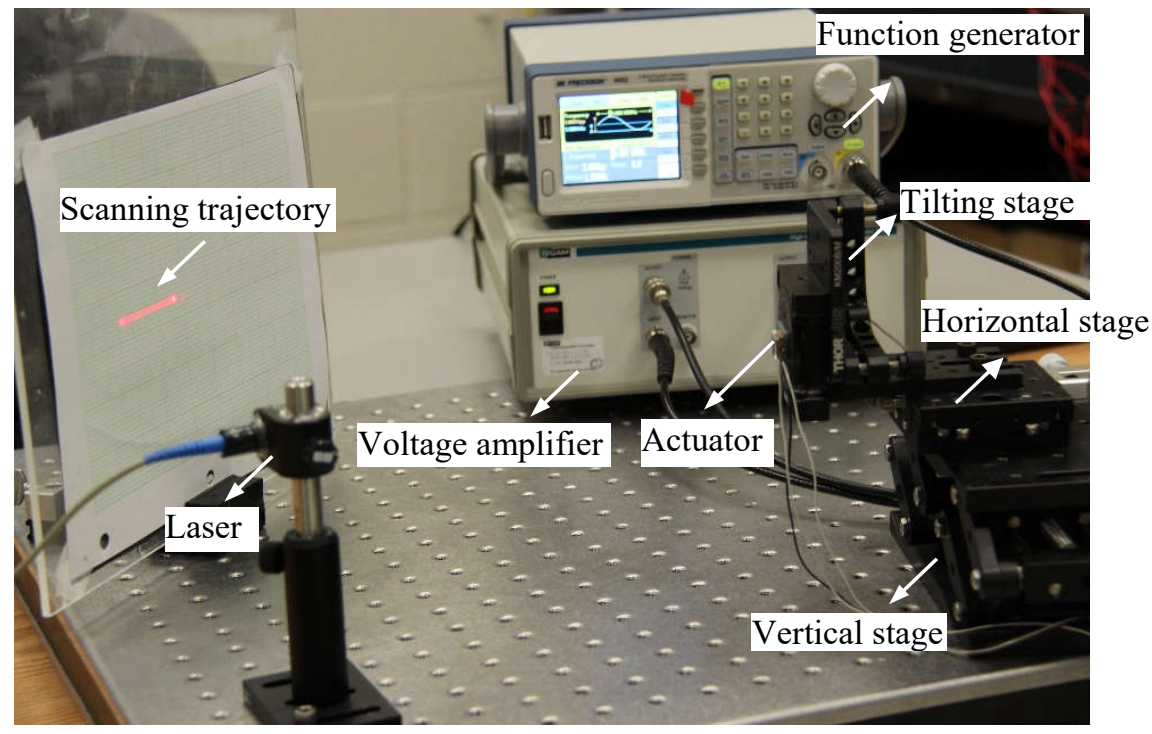

Fig. 3.12 Experiment setup for measuring the rotation of the actuator.

The driving voltage varies from 0 to $150 \mathrm{~V}$. The rotation angles are shown in Fig. 3.8, which shows that the actuator is able to achieve a mechanical rotation of $7.65^{\circ}$. Multiple actuators $(\geq 3)$ are measured under the same setup and it has been proved that the rotation angles are consistent with different actuators fabricated in the same run. The discrepancy between experimental results and model results at $150 \mathrm{~V}$ is $11.8 \%$. which is possibly attributed to the following sources: 1) The geometry is approximated in 2D electric field simulation when simulating the middle part of the actuator; 2) The rotation finger tips, the common beam (see Fig. 3.1(b)) and the part of the middle 
beam beyond the location aligned with the other rotation finger tips are not included in the model when calculating the total torque. That lowers the rotation torque and then rotation angle in the model results; and 3) In the measurement setup the reflected laser beam cannot be perfectly perpendicular to the display screen, which causes error of increasing the measured rotation angle.

\subsubsection{Dynamic performance}

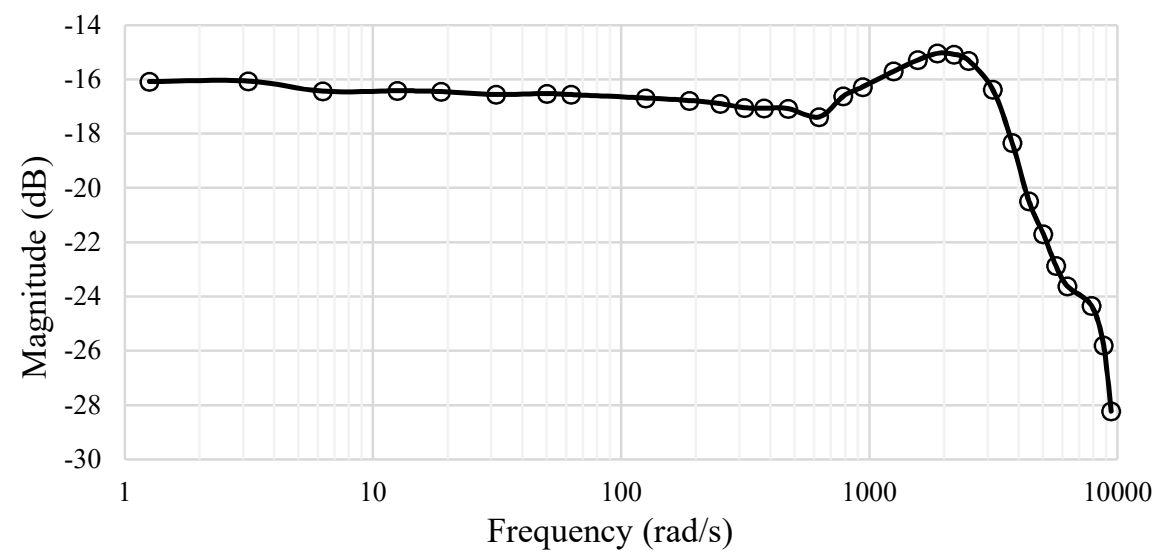

(a)

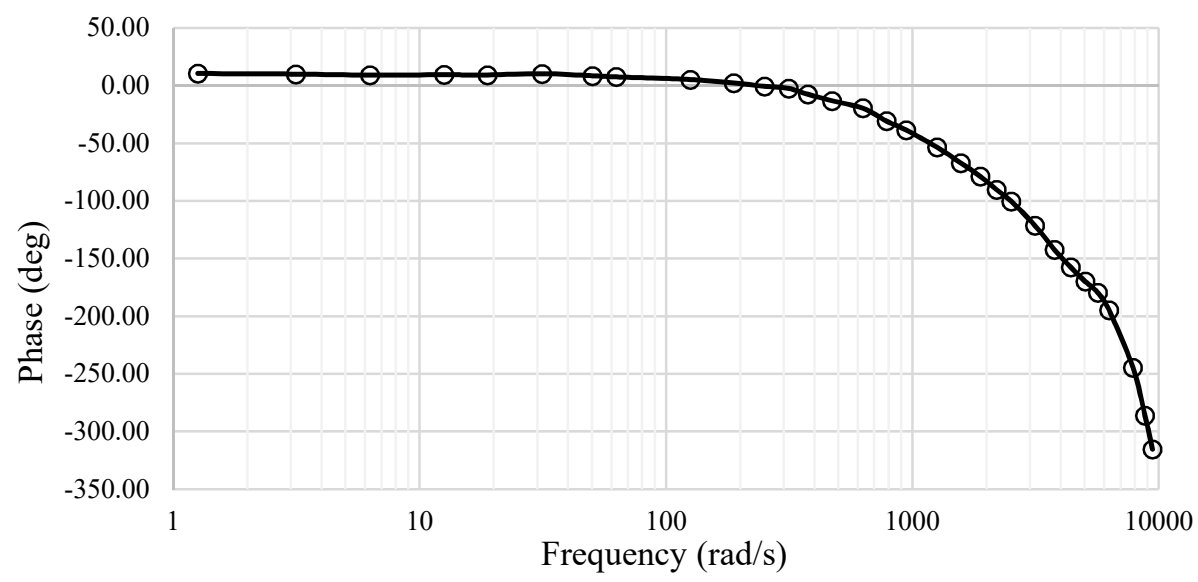

(b)

Fig. 3.13 Bode plot of the two-width finger actuator (a) Magnitude response (b) Phase angle response.

In dynamic test, sinusoidal driving voltages range between $10 \mathrm{~V}$ to $60 \mathrm{~V}$ with different frequencies are applied to the TWF actuator. The response of the laser displacement is measured using PSD similar to the measurement performed in chapter 2 for TRF actuator. The Bode plot is shown in Fig. 3.13. The input driving voltage signals and the output voltage signals form the PSD 
are used to construct the bode plot. The underdamped resonant frequency of the actuator is around $300 \mathrm{~Hz}$ smaller than the simulation result which is $392 \mathrm{~Hz}$ without considering the air damping effect. It has been analyzed in section 3.3.2 that the deflection of the middle beam is slightly different from the other fingers. The measured frequency response is only for the middle beam and the other fingers will have a slight different frequency response.

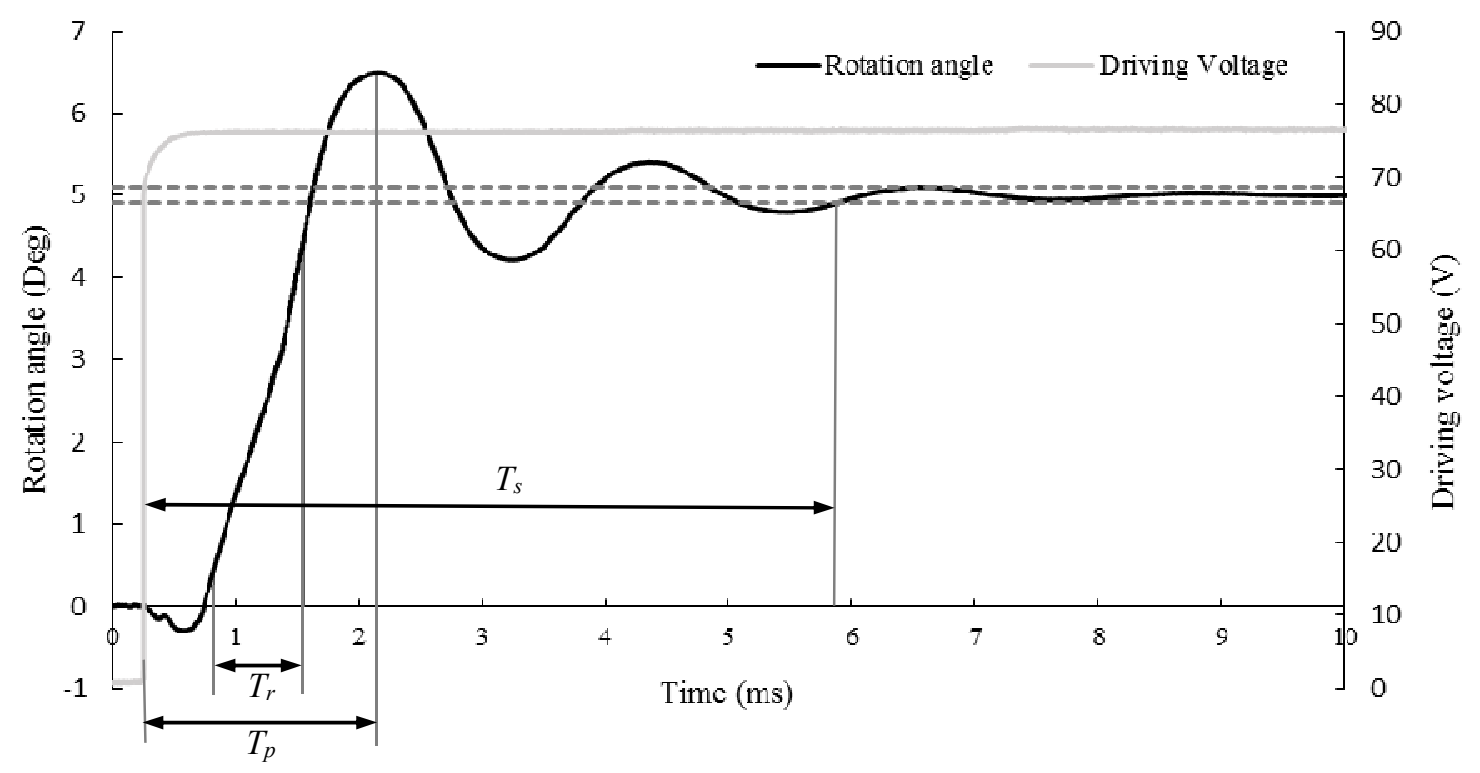

Fig. 3.14 Step response of the actuator from 0 to $5^{\circ}$ for a driving voltage of $76 \mathrm{~V}$.

The step response is also measured using the same setup as the frequency response. The PSD is able to measure the position of the reflected laser beam on its sensing area in real time. Then the real time rotation angle of the actuator can be calculated by knowing the displacement of the laser spot on the PSD and the distance between PSD and actuator. When the driving voltage is $76 \mathrm{~V}$, the mechanical rotation angle is $5^{\circ}$ and the laser spot almost reaches the edge of the PSD sensing area $(10 \mathrm{~mm} \times 10 \mathrm{~mm})$. If the driving voltage is greater than $76 \mathrm{~V}$, the actuator rotates more and the reflected laser beam moves out of the PSD sensing area. Thus the step response measurement using PSD is limited to $76 \mathrm{~V}$. In static rotation measurements, the laser beam is displayed on a grid paper without moving limitation. The step response is shown in Fig. 3.14. At the beginning of the actuation, the rotation angle has a small negative undershoot. This is due to the possible fact that the system is a non-minimum phase system. The rise time $(10 \% \sim 90 \%$ of the final steady state value), peak time and settling time (2\% steady state error) are denoted as $T_{r}, T_{p}$ and $T_{s}$ which are $0.7 \mathrm{~ms}, 2.0 \mathrm{~ms}$ and $5.7 \mathrm{~ms}$ respectively. The percentage overshoot is $29.8 \%$. The simulation shows that the middle beam deforms in dynamic vibration, i.e., bending along the length relative to its 
root end which connects to the common beam due to the large moment of inertia of the mirror plate. Therefore the step response performance also includes the effect due to the dynamic deformation of the middle beam and mirror plate structure.

\subsection{Summary}

An electrostatic repulsive-torque actuator with two-width fingers is presented in this chapter. It has a simple two-layer structure and can be fabricated using two-layer surface micromachining process. The actuator can generate large out-of-plane rotation and avoid the "pull-in" instability. The model is developed. Prototypes are fabricated and tested. Experimental results show that the actuator is able to achieve a static mechanical rotation of $7.65^{\circ}$, which is much larger than that of the previously reported ORF actuator of the same size and stiffness. Also the roation angle of the presented acuator is either better or comparable to those of reported out-of-plane combdrive actuator. For example, a maximum rotation angle of $6.2^{\circ}$ is measured with the combdrive actuated micromirror in [57]. The micromirror driven by the combdrive actuator can rotate $2.3^{\circ}$ in [23]. However the reported comb drive actuated micromirrors need microfabrication processes which are more complex and costly than the two-layer surface micromachining process used to fabricate the actuator presented in this chapter. The step response is measured for the two-width finger actuator with settling time of $5.7 \mathrm{~ms}$ for a mechanical rotation of $5^{\circ}$. 


\section{Chapter 4 Micromirror driven by the two-row interdigitating-finger actuator for vector display}

Section 4.1 is based on Section V of the following published paper:

Chao Fan and Siyuan He, "A two-row interdigitating-finger repulsive-torque electrostatic actuator and its application to micromirror vector display", IEEE Journal of Microelectromechanical Systems, vol. 24, pp. 2049 - 2061, 2015.

From the testing, it has been proved that the fabricated TWF actuator is able to achieve about $36 \%$ larger rotation angle than that of the ORF actuator of the same size at $150 \mathrm{~V}$. Also the fabricated TRF actuator is able to improve the rotation angle by nearly $100 \%$ over the ORF actuator of the same size at $150 \mathrm{~V}$. Since the two-row repulsive-torque electrostatic actuator presented in Chapter 2 is able to achieve larger rotation angle than the two-width repulsive-torque electrostatic actuator presented in Chapter 3, it is used to drive a 2D micromirror in this chapter which is the second objective of the thesis. Four TRF actuators are used to integrate with a mirror plate to form a $2 \mathrm{D}$ scanning micromirror. The working principle of the micromirror is introduced. The micromirror is fabricated using PolyMUMPs and the performance of the micromirror is tested and compared with the previously presented micromirror based on ORF actuators. Then the static (rotation angle) and dynamic performance (step and frequency response) of the micromirror driving by a proposed driving method has been tested.

\subsection{Micromirror for vector display}

\subsubsection{Micromirror driven by the TRF actuator}

Since the two-row interdigitating-finger actuator has the larger out-of-plane rotation between the two actuators developed in chapter 2 and 3 , it is used to actuate a 2D scanning micromirror. Four identical TRF actuators are connected to the peripheries of a micromirror plate through the actuators' middle beams and connection springs as shown in Fig. 4.1. It is noted that the Poly0 layer is not shown in Fig. 4.1. The 4 actuators of the micromirror can be divided into 2 groups, as shown in Fig. 4.1. A coordinate system with the origin located at the center of the micromirror plate can be built. 4 actuators are names as $A_{1}, A_{2}, A_{3}$ and $A_{4}$. Actuators $A_{1}$ and $A_{3}$ are one group 
which drives the micromirror plate to rotate with respect to y-axis. Actuator $A_{2}$ and $A_{4}$ are the other group which drives the micromirror plate to rotate with respect to x-axis. Since both actuators in the same group can only drive the micromirror plate on that side out of the plane, the rotation of the micromirror plate is achieved by the out-of-plane displacement difference of the connection point with the connection springs which is caused by the out-of-plane rotation of the actuators. Due to the fact that the rotation angle of each actuator can be controlled independently by its driving voltage, the rotation angle of the micromirror can also be controlled to continuously change and is able to sustain at any achievable angle. This type of micromirror is also called quasistatic micromirror. At last the combination of two axis rotation of the micromirror can steer the reflected laser beam to scan through a 2D area shown in Fig. 4.3. There are several driving methods of the micromirror, the one adopted in this thesis is shown in Eqs. (4.1) (4.4)

$$
\begin{aligned}
& V_{1}=V_{0}+V_{\text {bias_ }} a \\
& V_{3}=V_{0}-V_{\text {bias_ }} a \\
& V_{2}=V_{0}+V_{\text {bias_ }} b \\
& V_{4}=V_{0}-V_{\text {bias_ }} b
\end{aligned}
$$

Where $V_{1}, V_{2}, V_{3}$ and $V_{4}$ are the voltages applied to actuators 1,2,3 and 4 respectively. $V_{0}$ is the base voltage, e.g., $50 \mathrm{~V}$. $V_{\text {bias } a}$ and $V_{\text {bias__ }}$ are biased voltages ranging from $-50 \mathrm{~V} \sim 50 \mathrm{~V}$. Actuators 1 and 3 are in one group and subject to bias voltage of the same magnitude with opposite sign. Actuators 2 and 4 are in one group and subject to bias voltage of the same magnitude with opposite sign. The resultant different heights of the tips of the middle beams determine the orientation of the micromirror. Fig. 4.1 shows a few examples of different orientations of the mirror plate realized by varying the bias voltage. The dimensions of each of the four actuators such as the finger width, lower/upper anchoring spring stiffness, connecting spring stiffness, etc. can be adjusted according to the display system requirements such as the scanning area size, scanning speed, driving voltage and resistance to vibration, etc. The dimensions of the micromirror are shown in Table 4.1. The prototype of the micromirror is fabricated using PolyMUMPs as shown in Fig. 4.2 (a). Fig. 4.2 (b) shows packaged micromirror. 
Table 4.1 Dimensions of the micromirror (Unit: $\mu \mathrm{m}$ )

\begin{tabular}{cccccccccc}
\hline \hline $\begin{array}{c}\text { Micromirror } \\
\text { overall dimension }\end{array}$ & $\begin{array}{c}\text { Mirror } \\
\text { plate } \\
\text { diameter }\end{array}$ & $\begin{array}{c}\text { Actuator } \\
\text { width (Each) finger width }\end{array}$ & $\begin{array}{c}\text { Ringer } \\
\text { width }\end{array}$ & $\begin{array}{c}\text { Middle } \\
\text { beam } \\
\text { width }\end{array}$ & $\begin{array}{c}\text { Lower rotation } \\
\text { finger length }\end{array}$ & $\begin{array}{c}\text { Upper } \\
\text { rotation } \\
\text { finger length }\end{array}$ & $\begin{array}{c}\text { Middle } \\
\text { beam } \\
\text { length }\end{array}$ & $\begin{array}{c}\text { Connecting } \\
\text { spring width }\end{array}$ & $\begin{array}{c}\text { Lower/upper } \\
\text { anchoring } \\
\text { spring width }\end{array}$ \\
\hline $3317 \times 3317$ & 1000 & 2460 & 40 & 50 & 50 & $\begin{array}{c}200(150 \text { the } \\
\text { end fingers })\end{array}$ & 604 & 842 & 8 \\
\hline
\end{tabular}

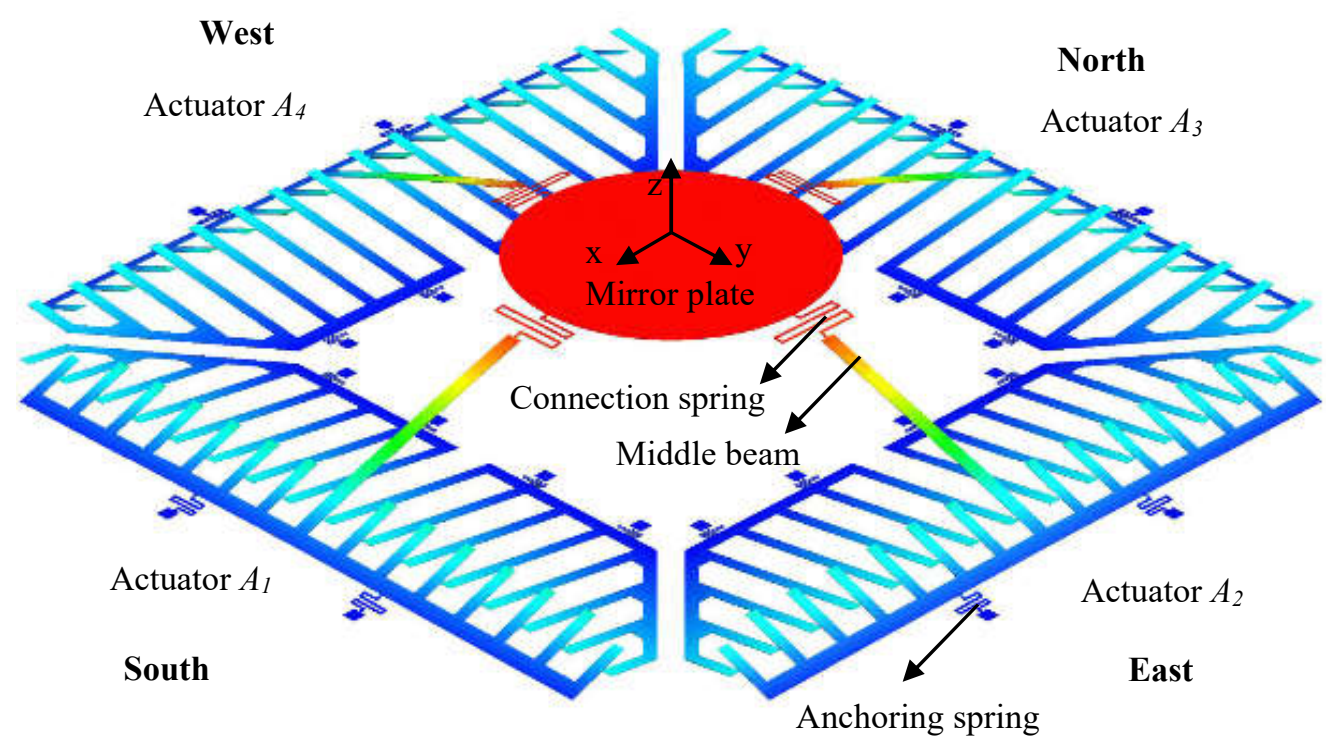

(a) $V_{\text {bias_a }}=0 \quad V_{\text {bias_ } b}=0$

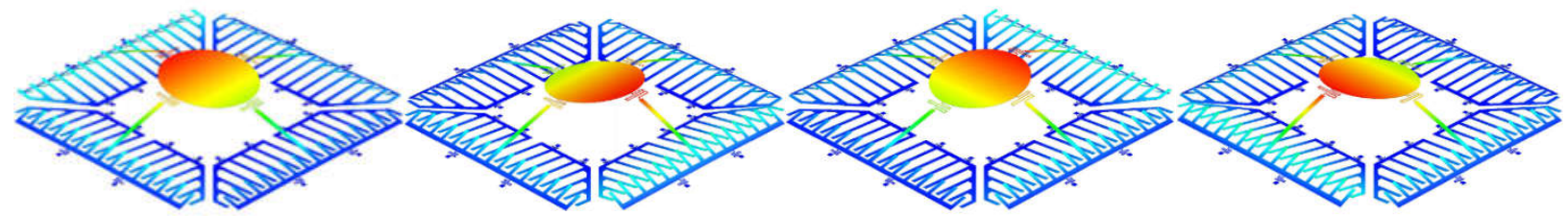

(b) $V_{\text {bias_a }}=0 V_{\text {bias_b }}<0$

(c) $V_{\text {bias_a }}=0 \quad V_{\text {bias_b }}>0$

(d) $V_{\text {bias_a }}<0 \quad V_{\text {bias_b }}=0$

(e) $V_{\text {bias_a }}>0 V_{\text {bias_ }}=0$

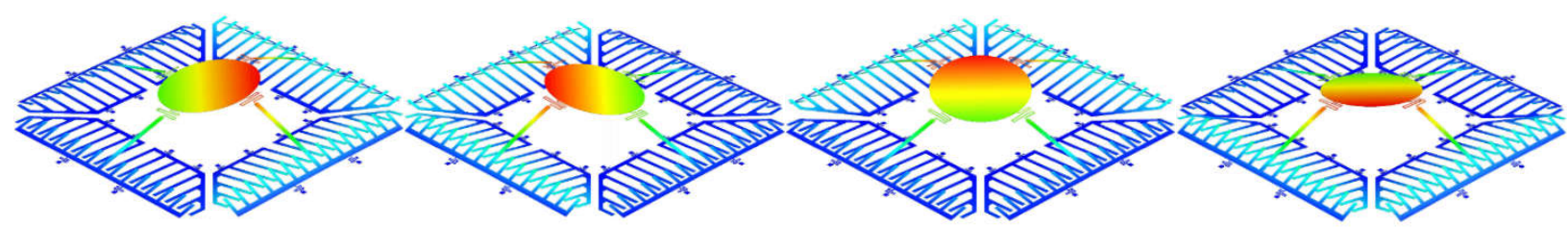
(f) $V_{\text {bias_a }}=-V_{\text {bias_b }} \quad V_{\text {bias } b}>0$
(g) $V_{\text {bias_a }}>0 V_{\text {bias_b }}=-V_{\text {bias_a }} a$
(h) $V_{\text {bias_a } a}=V_{\text {bias } b \text { b }}<0$
(i) $V_{\text {bias_a } a}=V_{\text {bias_ } b}>0$

Fig. 4.1 2D scanning micromirror based on TRF actuator. 


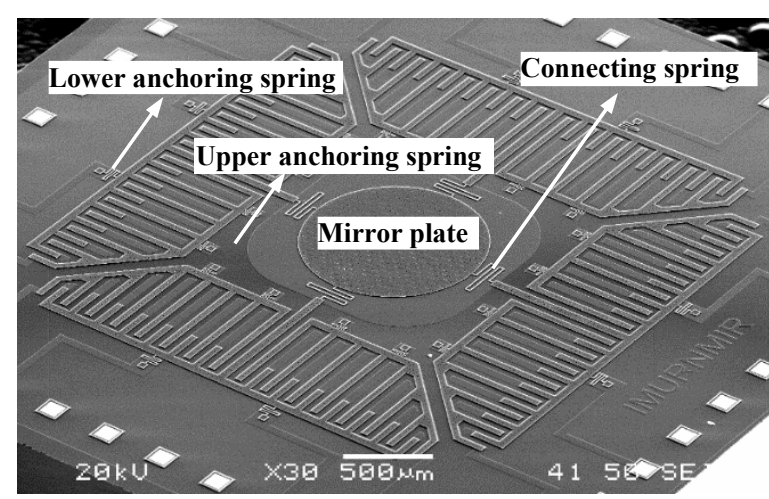

(a)

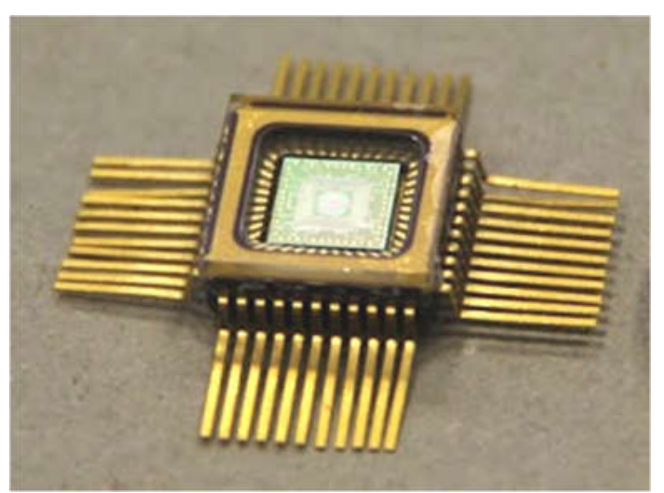

(b)

Fig. 4.2 The prototype of the micromirror for vector scanning display. (a) SEM picture of the micromirror (b) Micromirror packaged in CQFP44 chip with a protective glass bonded on the top.

\subsubsection{Micromirror based vector scanning display}

The micromirror can be used to manipulate a laser beam and generate laser scanning vector graphics, i.e., vector display. Different from the raster scanning display the laser beam of which scans through the whole display area line by line, the laser beam of the vector scanning display only scans through the outline of the desired patterns.

A micromirror based display system is shown in Fig. 4.3. A laser diode is used as the light source and the laser beam shoots onto the mirror plate which reflects and steers the laser beam to any desired direction with a high speed. The laser is modulated to be turned on and off with a relative high frequency so that the laser is only turned on for the desired visible trajectories. After reflected by the micromirror, a double side concave lens is used to 1) compensating for the convergence of the laser bean reflected by the micromirror plate due to its curvature. The concave lens is located about $4.5 \mathrm{~cm}$ from the micromirror to collimate the reflected laser beam; 2) Amplifying the scanning angle of the micromirror by about $3 \sim 6$ times. Thus by controlling the four actuators to drive the mirror plate for quasistatic 2D rotation and modulating the laser, the vector display can be realized. If a laser pattern is scanned periodically with a high refreshing rate, e.g., $\geq 30 \mathrm{~Hz}$, a steady laser pattern can be observed as shown in Fig. 4.3 (b). 


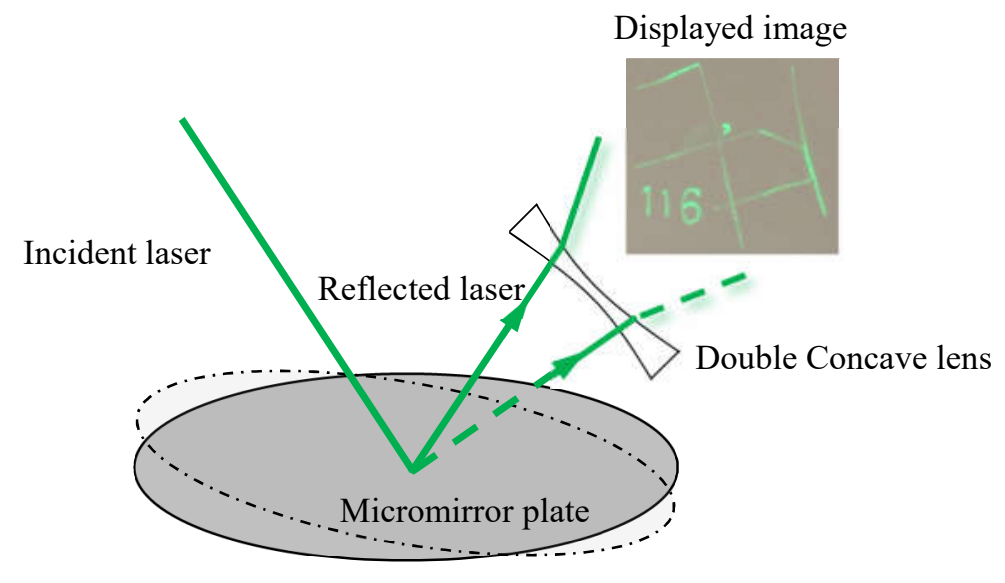

(a)

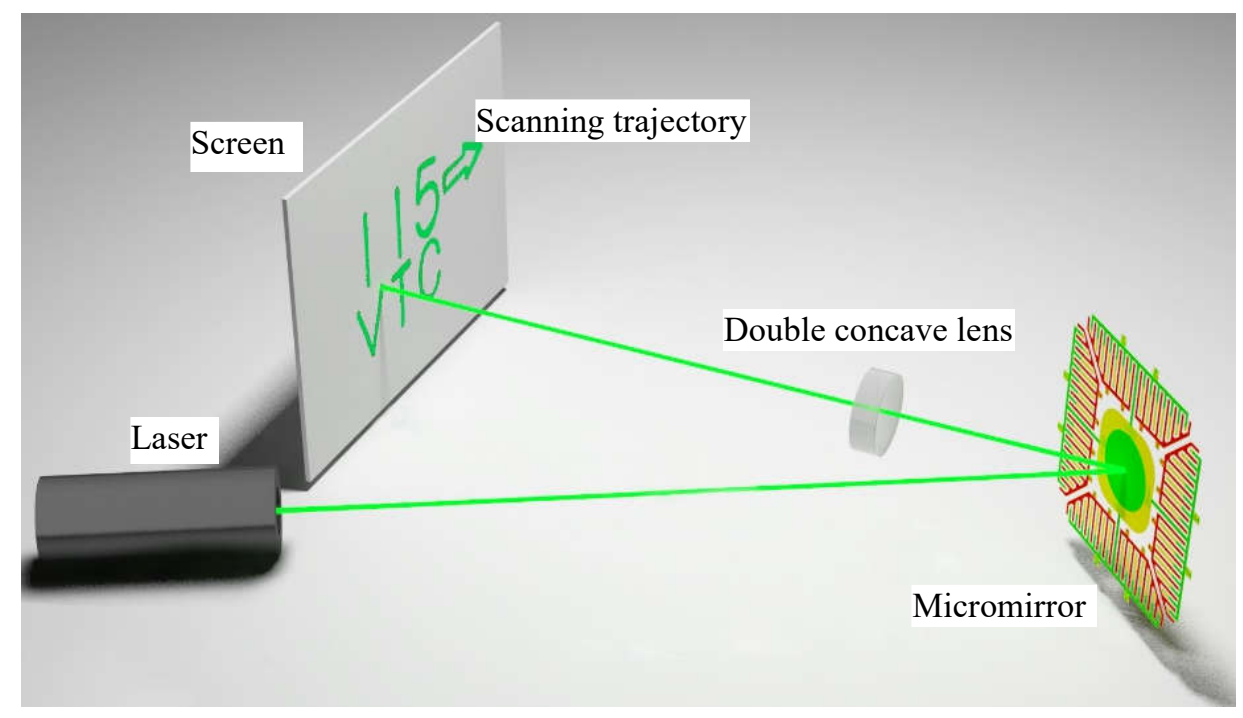

(b)

Fig. 4.3 Laser vector scanning display using the micromirror.

\subsection{Choosing double concave lens}

The double concave lens needs to be carefully selected to amplify the scanning angle and at the same time compensate for the curvature of the micromirror plate. It is desired that the lens can be selected as the one that is available on the market so that there is no extra cost for customization. The selection of the concave lens is presented in this section.

First, the model of the display system which is composed of collimated light source (laser), micromirror, a double side concave lens and a display screen is built in Zemax software shown in Fig 4.4. Zemax is a ray tracing software that can be used for both imaging and illumination systems. 


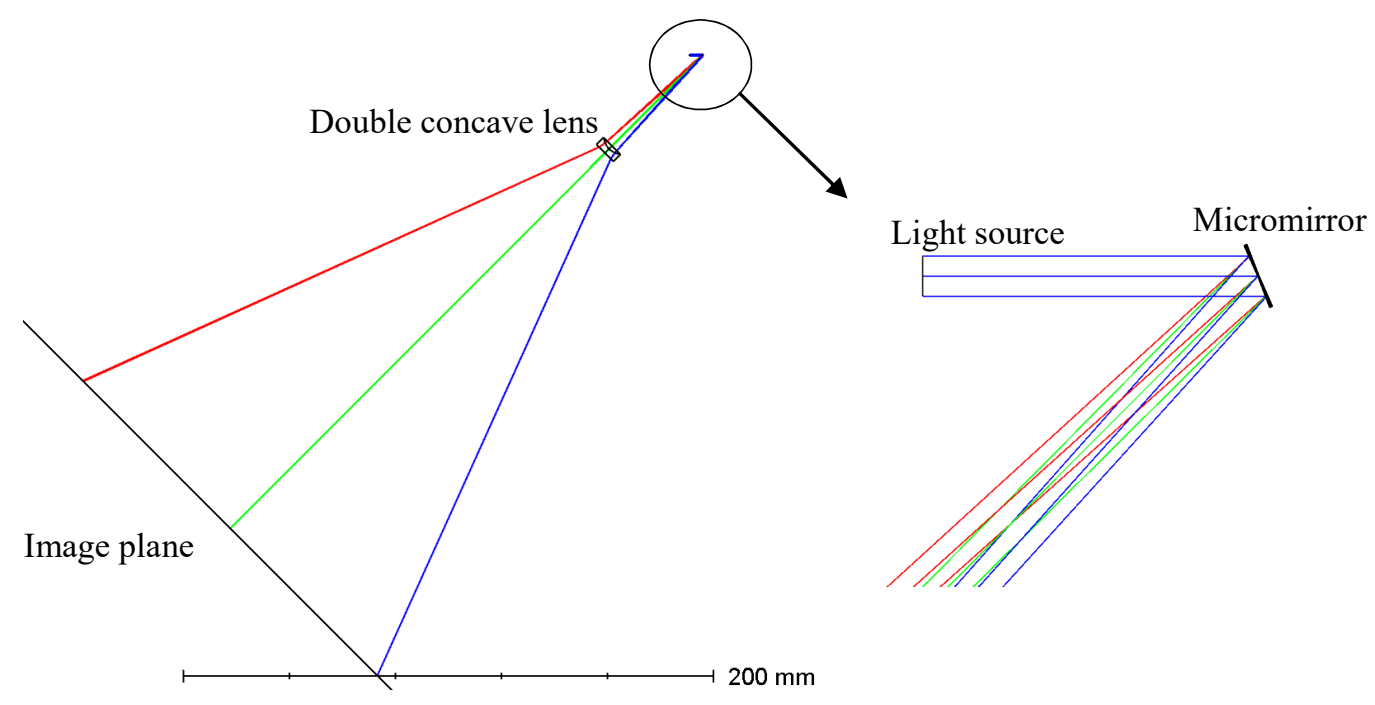

Fig. 4.4 Zemax model of the display system.

As shown in Fig. 4.4, a $1 \mathrm{~mm}$ diameter micromirror is positioned about $5 \mathrm{~mm}$ from the the light source and has about $45^{\circ}$ with the incident light. The radius of the curvature of the micromirror is set to be $125 \mathrm{~mm}$ which is measured using a 3D optical surafce profiler (zygo NewView 6000). The radius of the curvature changes in the range $90 \sim 135 \mathrm{~mm}$ due to different redidual strees gradiants in the fabrication process. One measured profile of the micromirror plate is shown in Fig. 4.5.
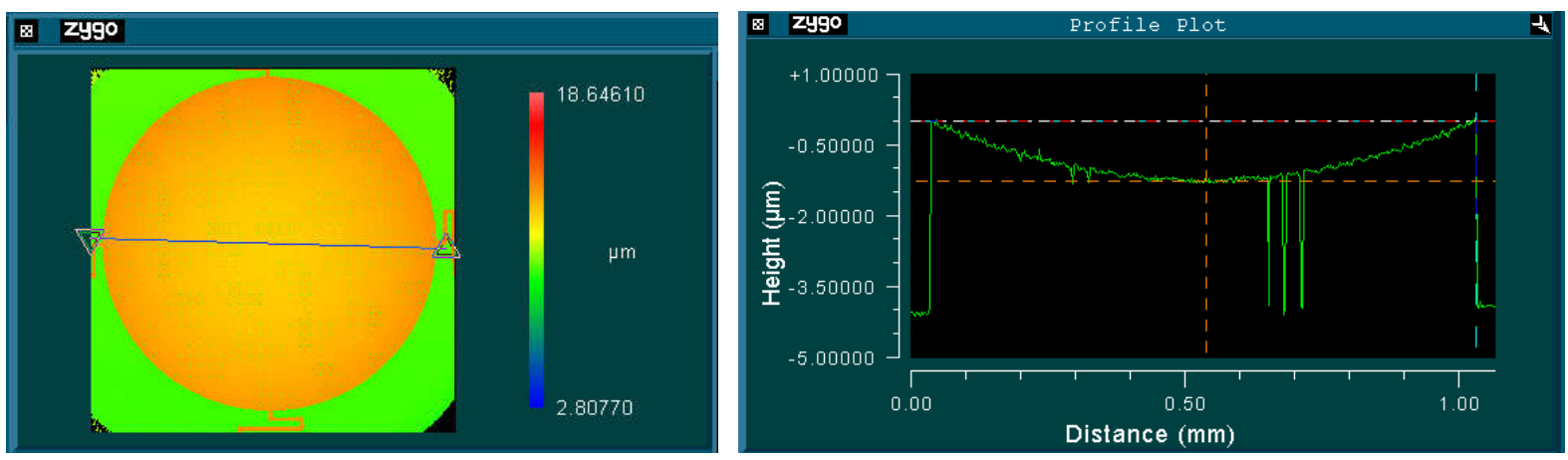

Fig. 4.5 Profile measure of the micromirror plate.

The micromirror is set to have a multiple tilt angles with respect to its center, i.e, $0^{\circ}, 1.5^{\circ}$ and $-1.5^{\circ}$ as shown in Fig. 4.6. There are three variables of the system, i.e., the distance from the double concave lens to the micromirror and the radius and thickness of the lens. The display scrren is set to be $200 \mathrm{~mm}$ away from the double concave lens. 
A series of simulations have been performed using Zemax with the parameters of the concave lens changing for each one. The parameters of the double concave lens from Edmund optics which is a large optical components provider in North America are used in the system. More specifically, for each lens, the thickness and the radius are obtained from the Edmund optics website and entered into the Zemax model. The local optimization solver is used to solve for the optimized distance between the lens and the micromirror. The wavefront is set as the criteria of the optimization function which optimize the optical path difference (OPD). The optical system is set to be in the afocal image space since the light does not focus to a plane, instead it is desired that the output light is nearly collimated at the image plane. As a result, the optimization function tries to make the light as collimated as possible when it reaches to the image plane.

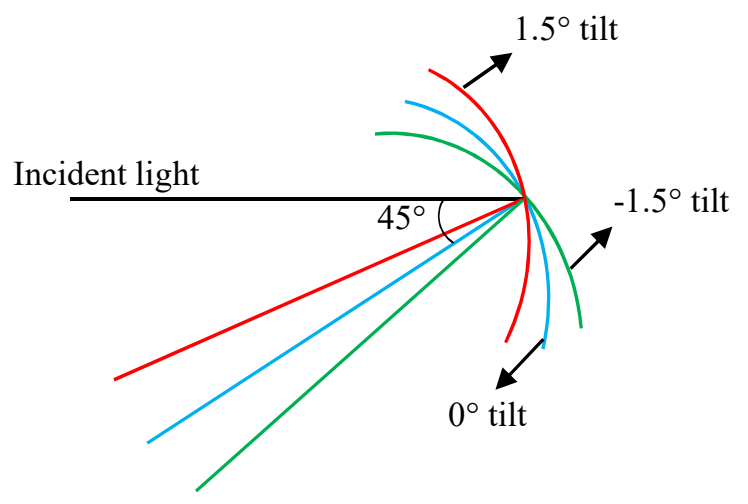

Fig. 4.6 Tilting of the micromiroror.

The lens used for simulation is shown in the table in Appendix C. The $\mathrm{MgF}_{2}$ coated antireflective (AR) double concave lenses are chosen to reduce the energy lose caused by the reflection. The reflectance is smaller than $1.75 \%$ persurface for the wavelength from $400 \sim 700 \mathrm{~nm}$. The AR lens provided by Edmound Optics has diameter of $6 \mathrm{~mm}, 9 \mathrm{~mm}, 12 \mathrm{~mm}, 20 \mathrm{~mm}$ and $25 \mathrm{~mm}$. Only the ones with diameter $9 \mathrm{~mm}$ and $12 \mathrm{~mm}$ are selected as the potential lens and are used for the Zemax optimization. Since it has been proved by Zemax simulation that most of $6 \mathrm{~mm}$ lens are too small for the incident scanning laser beams reflected from the micromirror. Also $20 \mathrm{~mm}$ and $25 \mathrm{~mm}$ diameter are too large and is not suitable for system integration. There are two criteria of choosing the lens. The first is the average divergence angles of the reflected laser beam of all the tilt angles. The drivergence of the laser beam determines the spot size on the image plane which 
futher determines the sharpness of the image. Smaller divergence angle of the laser beams after the lens is desired. The second criterion is the scanning distance on the display screen when the micromirror rotation from $-1.5^{\circ}$ to $-1.5^{\circ}$ along a single axis. A lens that has larger scanning distance is desired which can generate larger image on the display screen. The optimization results are shown in the table in Appendix C. It can be seen that the lens with parameter $12 \mathrm{~mm}$ Dia $\times$ $12 \mathrm{~mm}$ FL filfills the two criteria and is more siutable for the display. Its amplification factor can be estimated to be 5.7. However, since the actual micromirror curvature and and the distance of the lens from the micromirror changes, this factor may vary in the range from 3 to 6 .

\subsection{Performance of the micromirror}

\subsubsection{Static performance}

The mechanical rotation angle of the micromirror is measured using the setup on the optical breadboard shown in Fig. 4.7. A laser diode module with $532 \mathrm{~nm}$ wavelength is used to generate a collimated laser beam whose output spot size is about $1.2 \mathrm{~mm}$ diameter. Since the micromirror plate is $1 \mathrm{~mm}$, a beam reducer is used to reduce the diameter of the laser beam to about $0.6 \mathrm{~mm}$ while still maintains the beam collimated before it goes to the micromirror. The CQFP44 packaged micromirror is positioned in a micromirror fixture. Four linear stages are used to adjust the position of the micromirror plate so that the reflected laser is perpendicular with the optical breadboard. In this case, the plane formed by the incident laser beam and the reflected laser beam is almost parallel with the optical breadboard. In this case, the displacement of the laser beam on the screen is proportional to the rotation angle of the micromirror plate.

To obtain the mechanical rotation angle of the micromirror plate subjected to a certain combination of driving voltages (4 actuators), the corresponding displacement of the laser spot $d$ on the grid paper is recorded. The rotation angle of the micromirror $\theta$ is then calculated using Eq. (4.5) and illustrated in Fig. 4.8 (a).

$$
\theta=\arctan \left(\frac{d}{2 D_{s}}\right)
$$

Where $D_{s}$ is the distance of the gird screen to the center of the micromirror plate. 


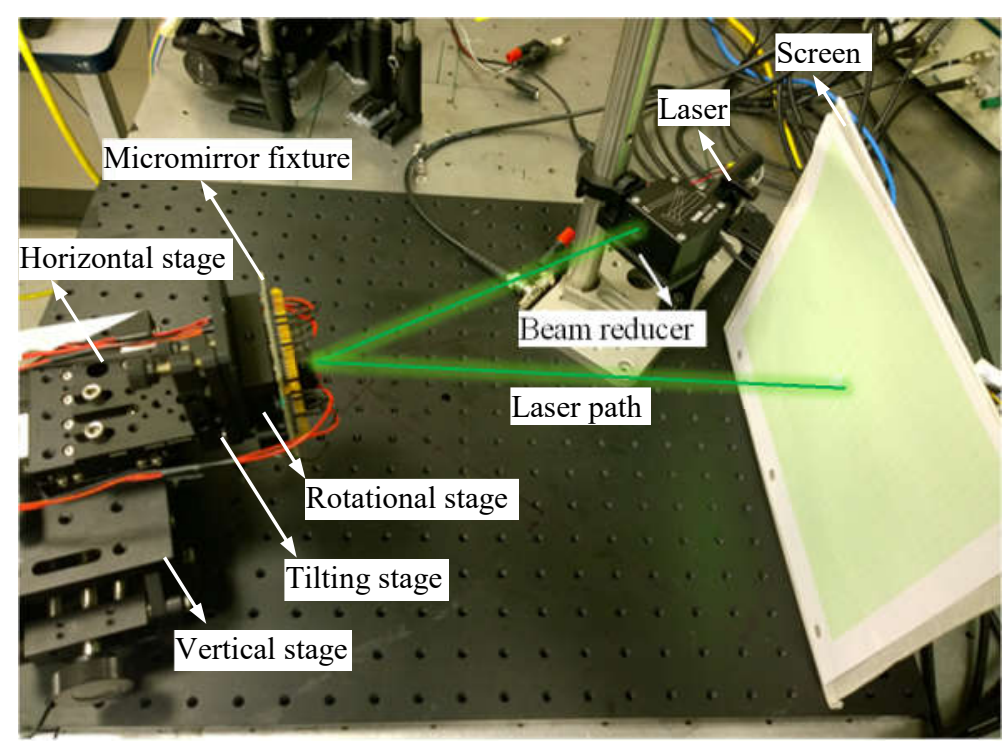

Fig. 4.7 Static performance test setup.

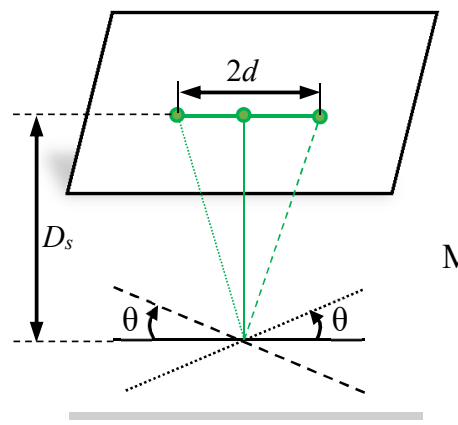

(a)

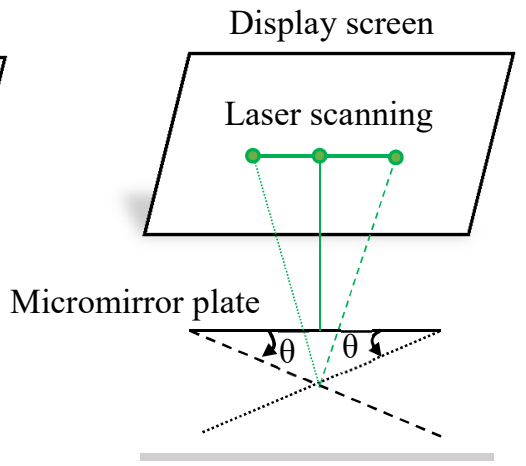

Underneath layer

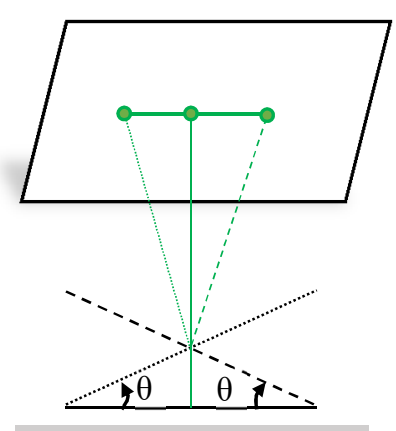

(c)

Fig. 4.8 Three driving approaches of the micromirror.

Because of the symmetrical structure of the micromirror, only the scanning angle of one axis of the micromirror is measured and the other axis shares the same rotation angle. Usually there are three different driving methods of the micromirror. They are illustrated in Fig. 4.8 by driving the micromirror to scan a horizontal line. 


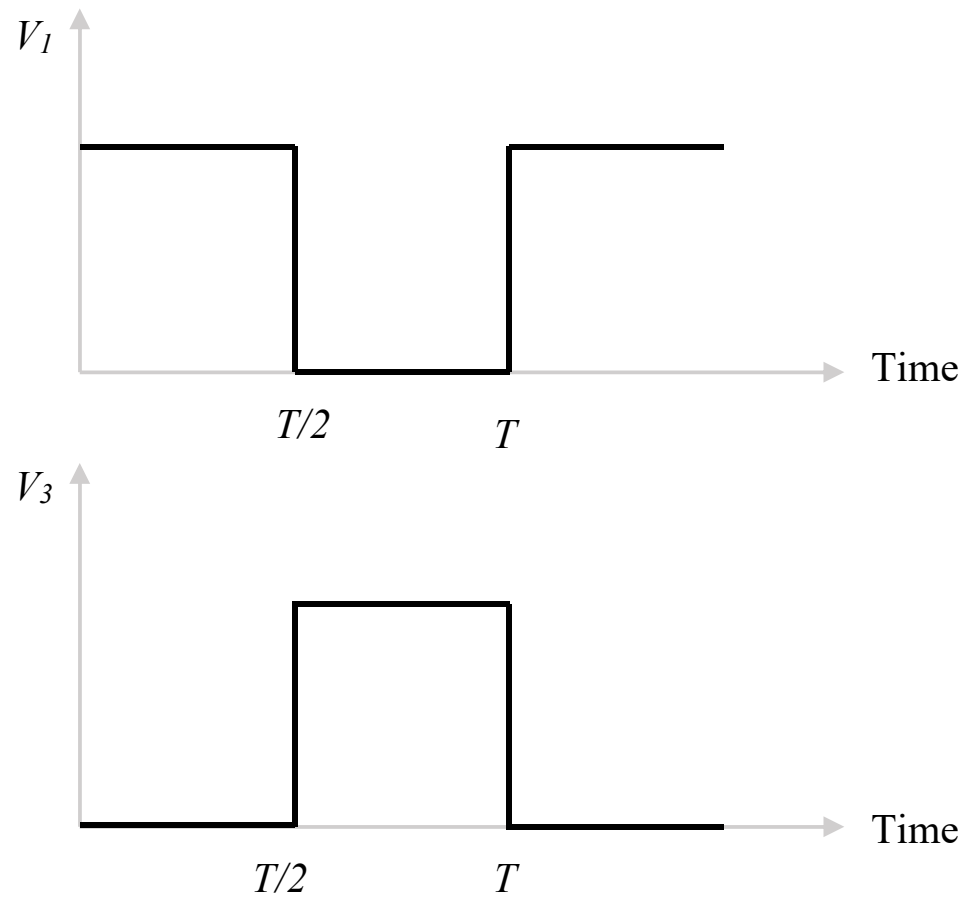

Fig. 4.9 Driving voltages for $V_{1}$ and $V_{3}$.

Different driving method has different balance position of the micromirror. The balance position of the micromirror is the position that the micromirror plate rotate around in the operation. When the micromirror is at the balance position, the corresponding reflected laser spot lies in the center of the display area. The first driving method is illustrated in Fig. 4.8 (a) and the driving voltages are defined by Eq. (4.1) (4.4). Specific to the one axis scanning defined in Fig. 4.8 (a), the base voltage $\left(V_{0}\right)$ is applied to actuators 2 and 4 while square wave voltages with half period phase difference (refer to Fig. 4.9) are applied to actuator 1 and 3. As a result, the balance position of the micromirror plate has a certain distance from its underneath layer and is defined by the base voltage. A higher base voltage leads to higher balance position of the micromirror plate. In operation, the movement of the micromirror plate can be approximated to be rotating around its center. The second driving method is shown in Fig. 4.8 (b). When the micromirror is scanning, a high voltage $V_{\text {high }}$ is applied to actuators 2 and 4 while square wave voltages with half period phase difference are applied to actuator 1 and 3 as shown in Fig. 4.9. As a result, the balance position of the micromirror plate is the position when $V_{\text {high }}$ is applied to all the actuators. Different from method 1, during the rotation the center of the rotation varies. The third driving method is shown in Fig. 4.8 (c). When the micromirror is scanning, zero voltage is applied to actuators 2 and 4 while square wave voltages with half period phase difference are applied to actuator 1 and 3 as shown in 
Fig. 4.8. As a result, the balance position of the micromirror plate is the position when no voltages are applied to the actuators and is the closest to its underneath layer among all three driving method. Similar to driving method 2 , the rotation center also changes during the rotation. The balance position of the micromirror can significantly affect the dynamic performance of the micromirror. If it is too close to the underlying layer, the squeezed film air damping will have great effect on the micromirror plate which behaves as an overdamped system. If it is too far away from the underlying layer, the air damping is greatly reduced and the micromirror behaves as an underdamped system with large overshoot. Due to the fact that the balance position of the micromirror using first driving method can be controlled by the base voltage $\left(V_{0}\right)$, by using it the balance position of the micromirror can be set to an optimized position so that the air damping is neither too large to lead to a sluggish response of the micromirror nor too small to result in a large overshoot. As a result, the first driving method is selected to be the driving method for the micromirror and the driving voltages of 4 actuators follows the equation expressed in Eqs. (4.1) (4.4). Different base voltages are used to drive the micromirror to scan a horizontal line. As shown in Table. 4.3 , the base voltages (the voltage applied to $V_{2}$ and $V_{4}$ ) vary from $30 \mathrm{~V}$ to $80 \mathrm{~V}$ with 10 $\mathrm{V}$ increment are used to drive the micromirror. The peak to peak voltage of $V_{l}$ and $V_{3}$ matches the corresponding base voltage to generate the maximum rotation angle.

Table 4.2 Test voltages (Unit: V)

\begin{tabular}{|c|c|c|c|c|}
\hline Test No. & $V_{1}$ & $V_{3}$ & $V_{2}$ & $V_{4}$ \\
\hline 1 & \multicolumn{2}{|c|}{ Square wave $0 \sim 60$} & 30 & 30 \\
\hline 2 & \multicolumn{2}{|c|}{ Square wave $0 \sim 80$} & 40 & 40 \\
\hline 3 & \multicolumn{2}{|c|}{ Square wave $0 \sim 100$} & 50 & 50 \\
\hline 4 & \multicolumn{2}{|c|}{ Square wave $0 \sim 120$} & 60 & 60 \\
\hline 5 & \multicolumn{2}{|c|}{ Square wave $0 \sim 140$} & 70 & 70 \\
\hline 6 & \multicolumn{2}{|c|}{ Square wave $0 \sim 160$} & 80 & 80 \\
\hline
\end{tabular}

The displacement of the reflected laser beam on the grid paper is manually marked on the grid paper only for the largest driving voltage shown in Table. 4.3, i.e., when $V_{l}$ and $V_{3}$ are applied $0 \sim$ $160 \mathrm{~V}$ square wave voltages. The rotation angles of the micromirror applied with $160 \mathrm{~V}$ peak to peak driving voltage, i.e., $\theta_{160 V}$, can be calculated using Eq. (4.5) by known the displacement (d) and the distance from the micromirror to the screen $\left(D_{s}\right)$. Then the grid paper is replaced by a 2D position sensing detector (PSD) which is an optical sensor and outputs the $\mathrm{x}$ and $\mathrm{y}$ coordinate of the laser spot on its sensing area as two voltages. The output voltages of the sensor is proportional 
to the location of the laser spot on its sensing area. The tests are then performed for each voltage combination listed in Table. 4.3. The corresponding laser displacements and together with their transient responses are captured by the PSD. The measured laser displacements of the tests $1 \sim 5$ using PSD is converted to the corresponding rotation angles of the micromirror using Eq. (4.6).

$$
\theta_{i}=\frac{d_{i}}{d_{160 V}} \theta_{160 V}
$$

Where $\theta_{i}$ and $d_{i}$ are the rotation angle of the micromirror and the displacement of the laser spot captured by the PSD when the peak to peak driving voltage is $i \mathrm{~V} . \theta_{160}$ is the micromirror rotation angle of test number 6 in Table 4.3 which was previously obtained. $d_{160}$ is the displacement of the laser spot captured by the PSD for test number 6 . The ratio between the rotation angles of tests 1 5 and $\theta_{160}$ is proportional to the ratio of their laser displacement. Hence the micromirror rotation angles of tests $1 \sim 5$ can be calculated using Eq. (4.6). The reason not to measure all the rotation angles manually by marking their displacements on the display screen is to reduce the measurement error. More specifically, when the driving voltages are small, the corresponding laser spot displacement on the screen is very small i.e., in a few millimeters. As a result, the error introduced by manually marking is usually large when the displacement is small. The error caused by manually marking is analyzed in Fig. 4.10 where the large grey circles represents the spot of the reflected laser beam on the display screen. It is desired that the marked points coincide with the center of the laser spot, which introduces no marking error. However, this will not happen in reality. On the other hand, the marked point is usually an arbitrary point within the diameter of the laser spot, shown as the green circle in Fig. 4.10. The maximum possible error happens when the marked points are on the edges of both laser spots, shown as the red squares in Fig. 4.10. As a result, if the distance between the two marked points is relatively small, i.e., in a few millimeters, a significant error might be introduced. For large driving voltages such as the ones in test 6 in Table. 4.3, the displacement of the laser spot on the screen is relatively large, i.e., more than 18 $\mathrm{mm}$. The laser spot diameter on the display screen is approximately $2 \mathrm{~mm}$. In this case, the possible maximum percentage of error introduced is about $11 \%$. It is noted that the $11 \%$ error only happens when the marking lies on the edges of the both spots. Usually it is guaranteed in the marking process that the marked points always lie within the laser spot. As a result the introduced error is far less than $11 \%$ and can be neglected. The error introduced by marking will increase with the 
degrees of the laser beam displacement. By combining the manually marking and PSD measurement method presented above, the introduced error can be minimized.

Marked laser spot inside the laser spot

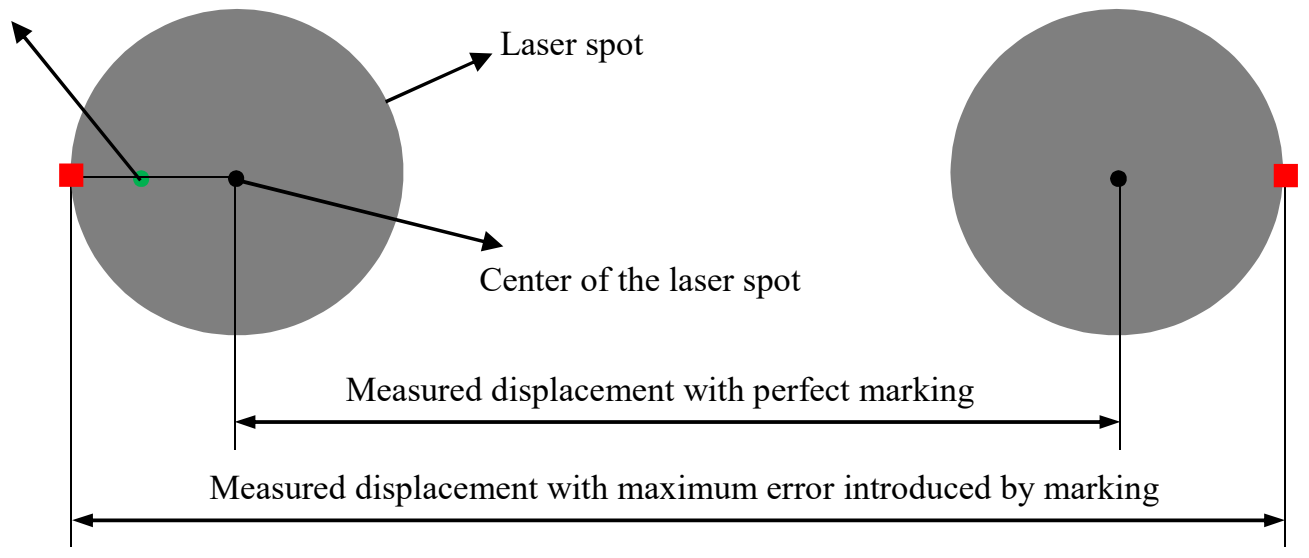

Fig. 4.10 Error introduced by manually marking.

The measured and calculated rotation angles for the tests listed in Table. 4.3 are shown in Fig. 4.11. The rotation angle here is the sum of the positive rotation angle and the absolute value of the negative rotation angle and is equal to twice of $\theta$ as shown in Fig. 4.8. From Fig. 4.11 it can be concluded that the scanning angle increases with the increase of the applied base voltage. The increasing rate decreases with the increase of the base voltage. Namely, when the driving voltage is relative high (near $200 \mathrm{~V}$ ) further increase of the voltage will not lead to significant increase of the rotation angle. On the other hand, high voltage may cause the breakdown of the insolation layer of the device. In our experiment, $50 \mathrm{~V}$ is selected as the base voltage to drive the micromirror which will lead to desired dynamic performance. The detailed reasons will be discussed in the dynamic test of the micromirror in section 4.3.2.

Then the rotation angle variations with respect to the changes of the bias voltages are studied in detail when the base voltage is $50 \mathrm{~V}$, as shown in Fig. 4.12. In Fig. 4.12, positive rotation angles represents rotations towards north, east, northeast or northwest (refer to Fig. 4.2). Negative values represent rotations towards south, west, southeast and southwest. Horizontal axis shows the magnitudes of biased voltages $V_{\text {bias_a }} a$ or $V_{\text {bias_b }}$. The applied biased voltages are the same as shown in Fig. 4.1 (b) $\sim($ h). In order to obtain the pure mechanical rotation angle, double side concave lens is not added for the measurement. From the testing results, the maximum mechanical rotation 
angle along north-south and east-west is about $1.1^{\circ}$ and the angle along northeast-southeast and northeast-southwest direction is about $1.5^{\circ}$ when the bias voltage is $50 \mathrm{~V}$.

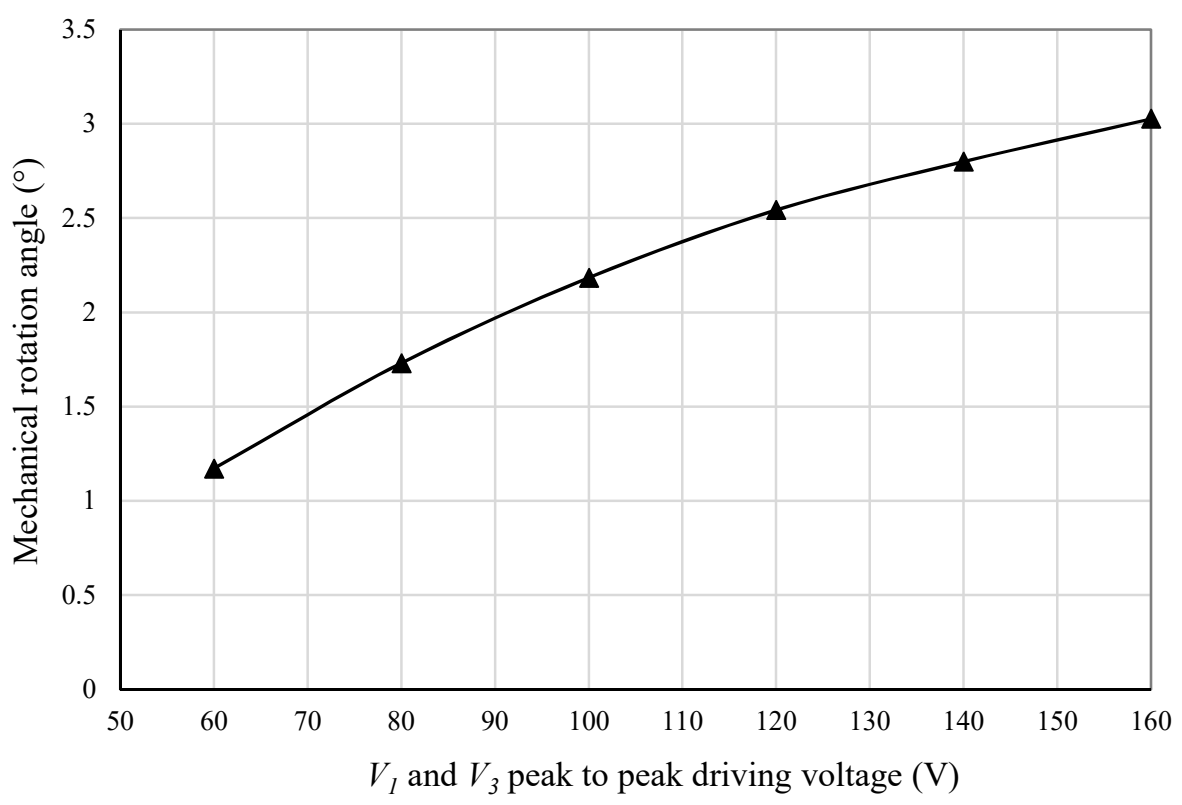

Fig. 4.11 Micromirror rotation angles under different base voltages.

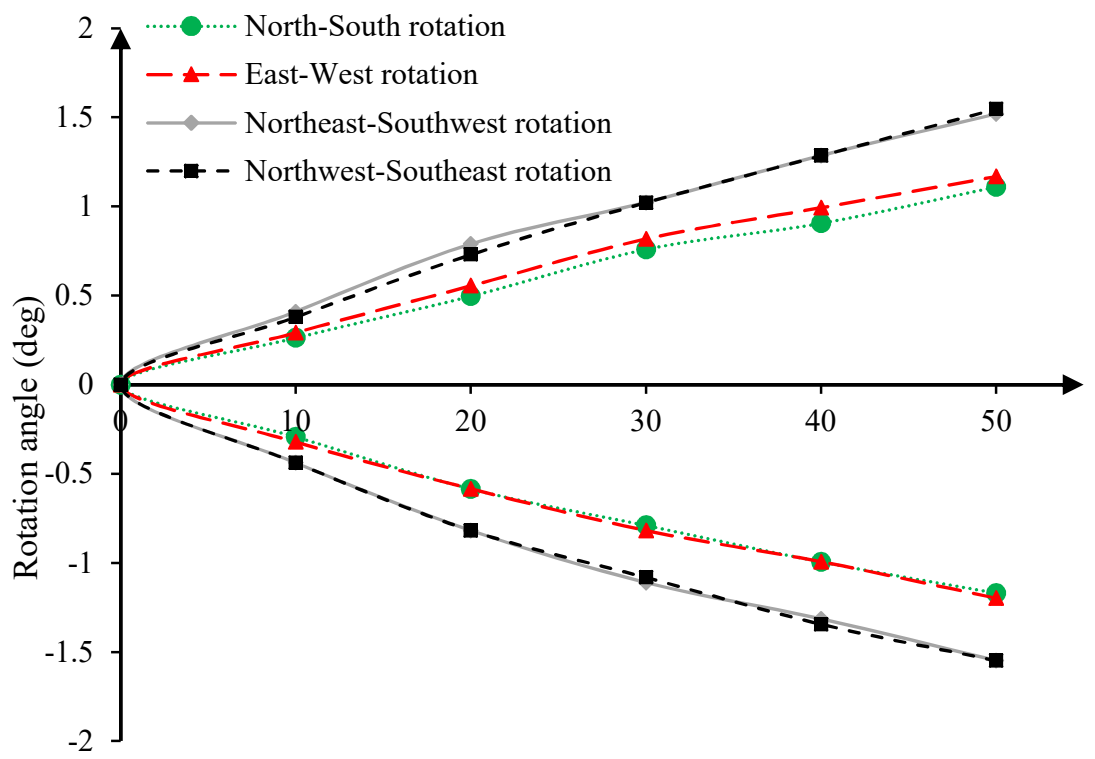

Bias voltage (V)

Fig. 4.12 Mechanical rotation of the micromirror. 


\subsubsection{Dynamic performance}

Table 4.3 Voltage combinations for step response test.

\begin{tabular}{|c|c|c|c|c|c|}
\hline & $V_{\text {bias } a}$ & $V_{I}$ & $V_{4}$ & $V_{2}$ & $V_{4}$ \\
\hline \multirow{5}{*}{$V_{\text {base }}=50 \mathrm{~V}$} & 10 & 40 & 60 & 50 & 50 \\
\cline { 2 - 6 } & 20 & 30 & 70 & 50 & 50 \\
\cline { 2 - 6 } & 30 & 20 & 80 & 50 & 50 \\
\cline { 2 - 6 } & 40 & 10 & 90 & 50 & 50 \\
\cline { 2 - 6 } & 50 & 0 & 100 & 50 & 50 \\
\hline base $=60 \mathrm{~V}$ & 10 & 50 & 70 & 60 & 60 \\
\cline { 2 - 6 } & 20 & 40 & 80 & 60 & 60 \\
\cline { 2 - 6 } & 30 & 30 & 90 & 60 & 60 \\
\cline { 2 - 6 } & 40 & 20 & 100 & 60 & 60 \\
\cline { 2 - 6 } & 50 & 10 & 110 & 60 & 60 \\
\hline \multirow{5}{*}{ base $=70 \mathrm{~V}$} & 60 & 0 & 120 & 60 & 60 \\
\cline { 2 - 6 } & 10 & 60 & 80 & 70 & 70 \\
\cline { 2 - 6 } & 20 & 50 & 90 & 70 & 70 \\
\cline { 2 - 6 } & 30 & 40 & 100 & 70 & 70 \\
\cline { 2 - 6 } & 50 & 30 & 110 & 70 & 70 \\
\cline { 2 - 6 } & 60 & 10 & 130 & 70 & 70 \\
\hline & 70 & 0 & 80 & 70 & 70 \\
\hline & 10 & 70 & 90 & 80 & 80 \\
\cline { 2 - 6 } & 20 & 60 & 100 & 80 & 80 \\
\cline { 2 - 6 } & 30 & 50 & 110 & 80 & 80 \\
\cline { 2 - 6 } & 40 & 40 & 120 & 80 & 80 \\
\cline { 2 - 6 } & 50 & 30 & 130 & 80 & 80 \\
\cline { 2 - 6 } & 60 & 20 & 140 & 80 & 80 \\
\cline { 2 - 6 } & 70 & 10 & 150 & 80 & 80 \\
\cline { 2 - 6 } & 80 & 0 & 160 & 80 & 80 \\
\hline
\end{tabular}

The transient response of the laser spot movement reflect the transient response of the micromirror and can be acquired by the PSD. 3 characteristics are used to evaluate the transient step response of the micromirrors which are rise time $\left(T_{r}\right)$, settling time $\left(T_{s}\right)$ and percentage of the overshoot $(O s)$. Rise time is the time for the response signal to travel from $10 \%$ to $90 \%$ of the final steady state. Settling time is the time for the response signal to be stabilized within $\pm 2 \%$ bandwidth of the final state value. Percentage of overshoot is defined as the ratio of the difference between the peak value of the response signal and the steady state value with the difference between the steady state value with the initial state value. 
The transient step response of the micromirror under different base voltages are tested. To make it easier to observe the performance, the micromirror plate is only controlled to perform a 1D scanning. The driving methods presented in Eq. (4.1) (4.4) are used to generate the driving voltages. For each test, first all the actuators are applied the base voltage and zero bias voltage is applied to all the actuators for a while which will stabilize the micromirror plate to its balance position. Then a non-zero bias voltage is applied to only one group of actuators, i.e., actuator 1 and 3, while the voltages of actuator 2 and 4 stay unchanged. As a result, the micromirror plate will rotate with respect to y-axis (shown in Fig. 4.1 (a)) from the balance position to a certain angle which is determined by the magnitude of the applied bias voltage. The voltage combinations used for tests are summarized in Table 4.4 and the performances are measured and analyzed.

The measured results of $T_{r}, T_{s}$ and $O s$ are shown in Figs. $4.12 \sim 4.14$. Fig. 4.12 shows the rise time variations of different base voltages with the changes of applied bias voltages. It verifies that lower base voltage leads to a sluggish response since the micromirror plate is close to its underlying layer and the air damping provides a significant resistance to the movement. With the increase of the base voltage, the balance position of the micromirror plate is farther away from the underlying layer and as a result, the response speed of the micromirror can be significantly improved. Also it can be concluded that for the same base voltage, the rise time reduces with the increase of the bias voltage. This is due to the fact that higher bias voltage will generate higher rotation torques which will lead to higher response speed. This effect is only significant when the base voltage is low such as $50 \mathrm{~V}$ and $60 \mathrm{~V}$. When the base voltage is high $(>60 \mathrm{~V})$, the improvement of the rise time by increasing the bias voltage is not much. However the fast response speed of high base voltage comes with a drawback of large percentage of overshoot as shown in Fig 4.14. This is attributed to the significantly weakened air damping. It can be seen that the percentage of the overshoot is about $40 \% \sim 50 \%$ when the base voltage is $80 \mathrm{~V}$, which is due to the reduced air damping. The percentage of overshoot reduces to nearly zero when the base voltage is reduced to $50 \mathrm{~V}$. As a result, even the response speed can be improved by increasing the base voltage, the accompanying large overshoot will cause vibration of the system, which washes away the benefit of fast response speed and leads to long settling time. The settling time of the tests shown in Table 4.4 is plotted in Fig. 4.15. It can be seen that the settling time of the response under base voltages $60 \mathrm{~V} \sim 80 \mathrm{~V}$ have a significant increase with the increase of the bias voltage, which is attributed to the vibration of the system which is caused by large overshoot and matches the overshoot plot shown in Fig. 4.14. 
Also it can be seen that the settling time of $50 \mathrm{~V}$ base voltage tests maintain a relative small value over the entire available bias voltage ranges which is mainly due to the consistency of its percentage of overshoot. It can be concluded that by using $50 \mathrm{~V}$ base voltage the micromirror has a consistent and relatively small settling time over the entire bias voltage range without overshoot. It comes with a cost of slow response speed (relative long rise time).

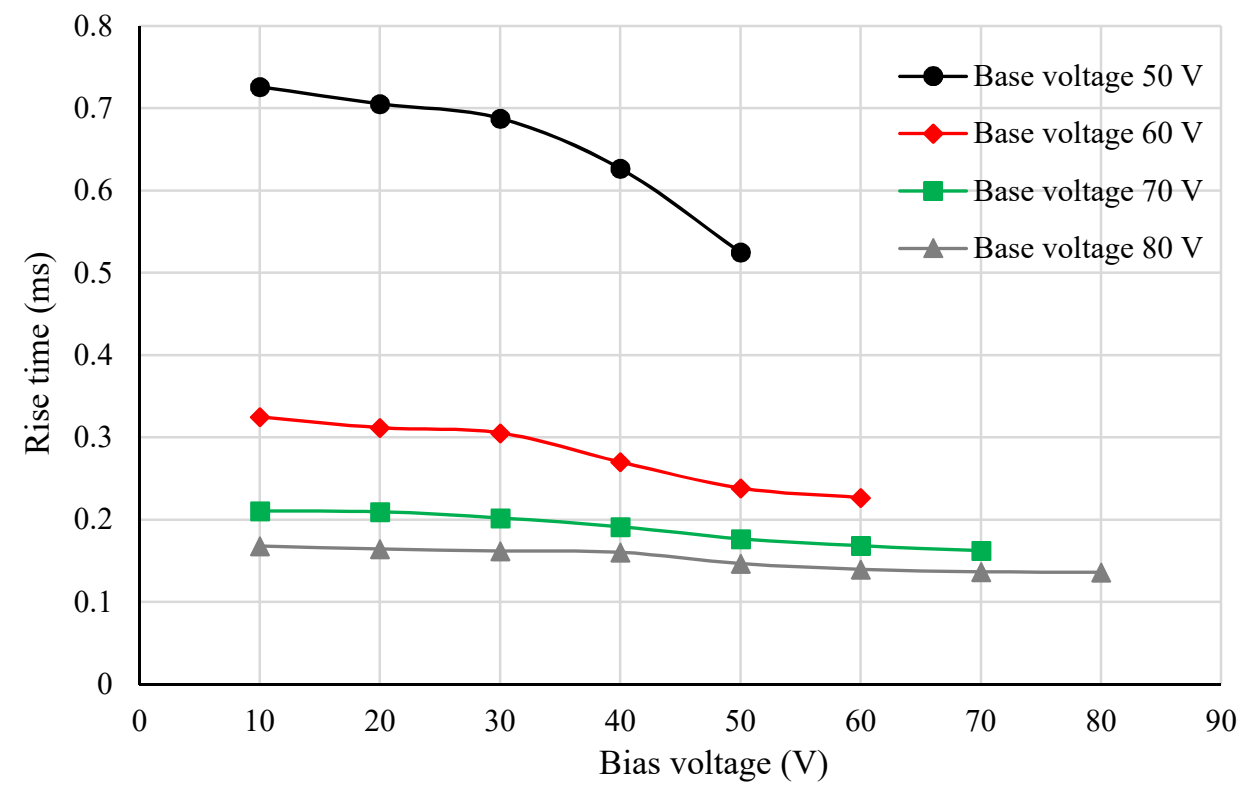

Fig. 4.13 Rise time of the micromirror under different base voltages.

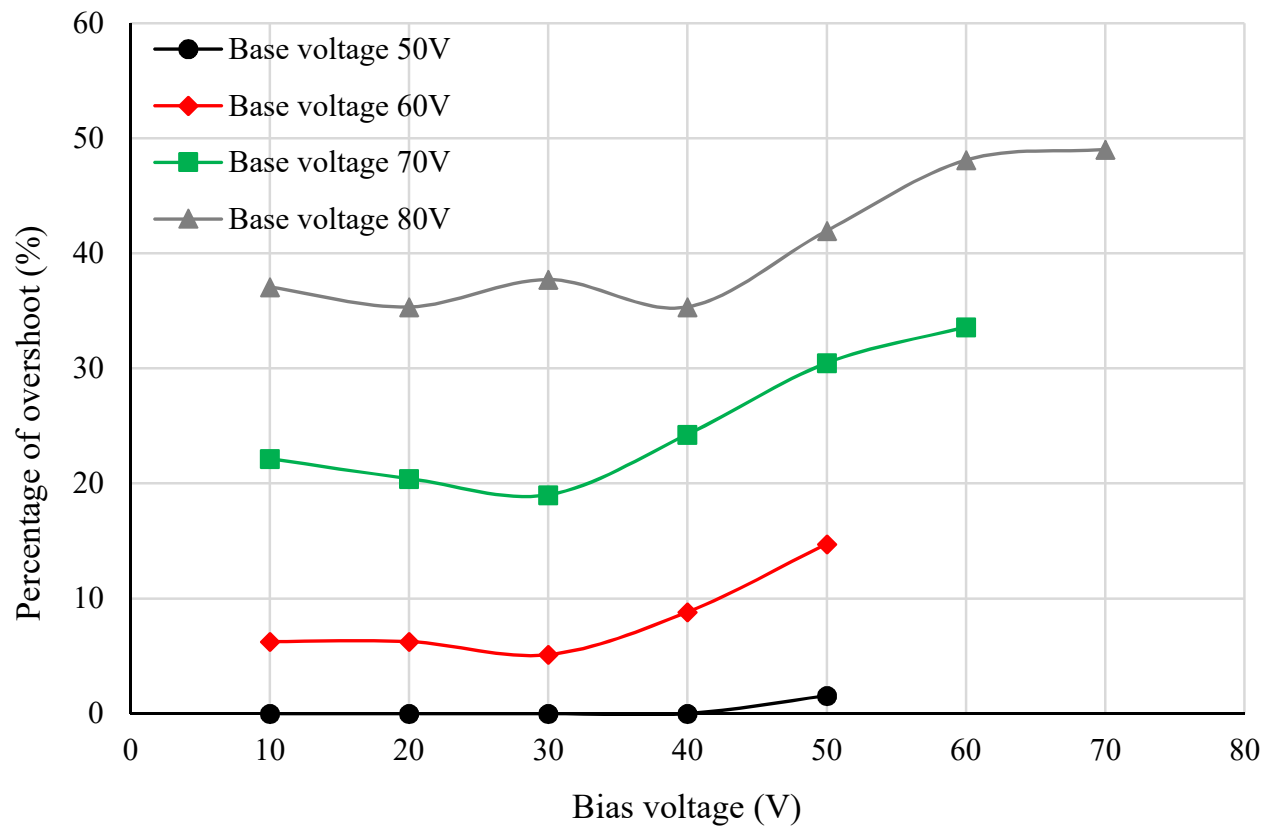

Fig. 4.14 Percentage of overshoot of the micromirror under different base voltages. 


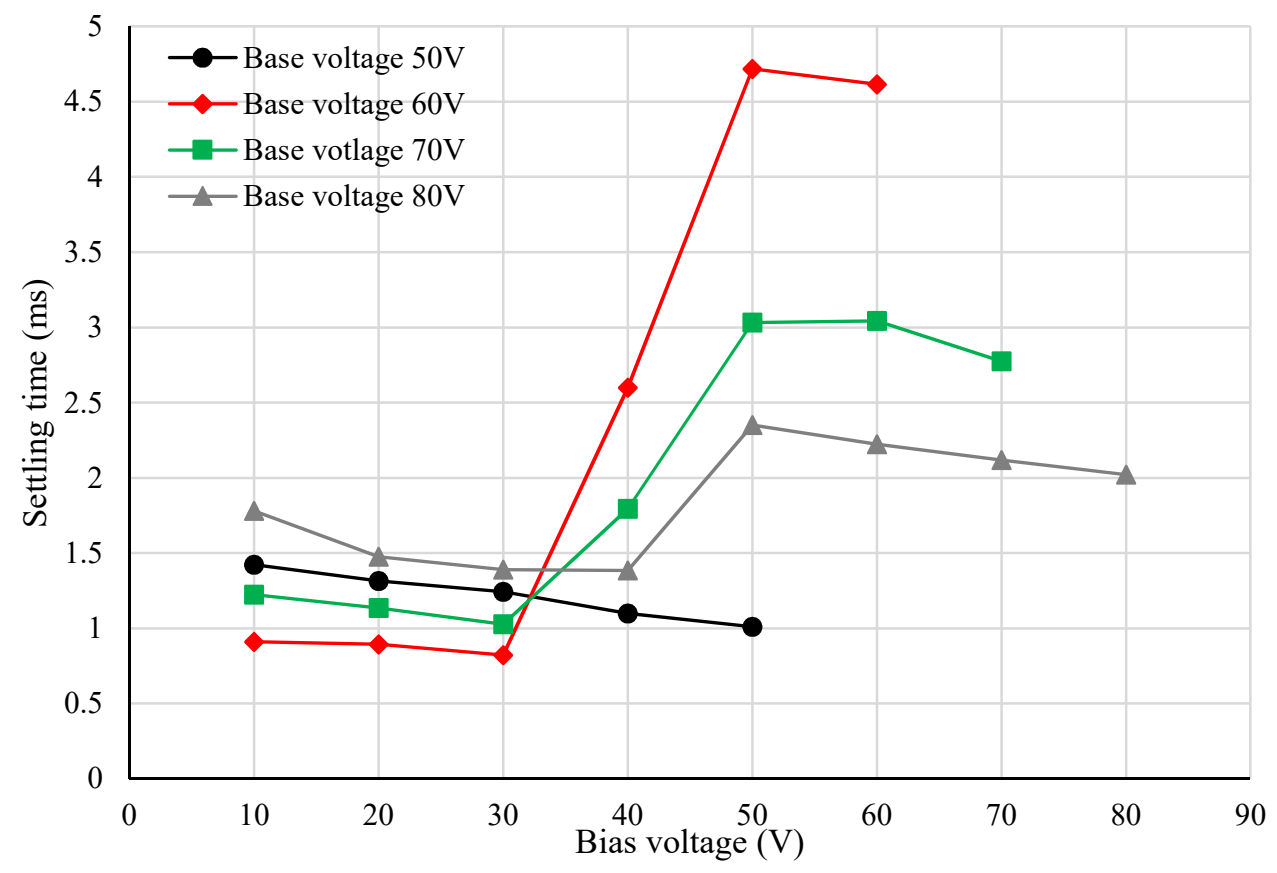

Fig. 4.15 Settling time of the micromirror under different base voltages.

For vector display application it is desired that the micromirror can achieve a large maximum rotation angle $\left(1^{\circ}\right.$ scanning angle, $\left.\pm 0.5^{\circ}\right)$ to have a large display area and meanwhile the transient response of the micromirror has small settling time and overshoot (less than 5\%) and fast response speed to improve the refreshing rate and then reduce the display distortion. Since $50 \mathrm{~V}$ base voltage leads to almost no overshoot and considering its consistent dynamic performance, it is the most suitable base voltage to drive the micromirror. The only defect of slow response speed can be improved using control approach which will be presented in Chapter 5.

The frequency response of the micromirror is also measured. The experiment setup is similar to Fig. 4.7 except that a PSD is used to acquire the movement of the reflected laser beam. The frequency response of the micromirror driven under two base voltages, i.e., $50 \mathrm{~V}$ and $70 \mathrm{~V}$, are measured separately for different bias voltages. The voltages applied to the micromirror is summarized in Table. 4.5 which are generated using Eq. (4.1) (4.4). For all the tests, the bias voltage $V_{\text {bias }} b$ for actuator $A_{2}$ and $A_{4}$ is zero therefore the DC base voltage is directly applied to them during the tests. While depends on different $V_{\text {bias }} a$ voltages, the sinusoidal voltages with different amplitudes are applied to actuator $A_{2}$ and $A_{4}$ in each test. 
Table 4.4 Test voltages (Unit: V)

\begin{tabular}{|c|c|c|c|c|c|c|c|}
\hline Test No. & $V_{\text {base }}$ & $V_{\text {bias } b}$ & $V_{2}$ & $V_{4}$ & $V_{\text {bias a }}$ & $V_{1}$ & $V_{3}$ \\
\hline 1 & \multirow{3}{*}{50} & \multirow{6}{*}{0} & 50 & 50 & 50 & $0 \sim 100$ & $100 \sim 0$ \\
\hline 2 & & & 50 & 50 & 25 & $25 \sim 75$ & $75 \sim 25$ \\
\hline 3 & & & 50 & 50 & 5 & $45 \sim 55$ & $55 \sim 45$ \\
\hline 4 & \multirow{3}{*}{70} & & 70 & 70 & 70 & $0 \sim 140$ & $140 \sim 0$ \\
\hline 5 & & & 70 & 70 & 35 & $35 \sim 105$ & $105 \sim 35$ \\
\hline 6 & & & 70 & 70 & 7 & $63 \sim 77$ & $77 \sim 63$ \\
\hline
\end{tabular}

For each test, two sinusoidal driving signals with frequencies varying from low to high and $90^{\circ}$ phase angle differences are applied to actuators $A_{1}$ and $A_{3}$. The corresponding responses of the reflected laser beam are acquired by the PSD. Bode plot is created using the recorded data and are shown in Figs. $4.16 \sim 4.18$. Some characteristic of the micromirror dynamic performance can be concluded as below.

(1) From the amplitude responses, it can be concluded that in low base voltage operation, i.e., smaller or equal to $50 \mathrm{~V}$, the micromirror appears to be an overdamped system. In high base voltage operation, i.e., larger or equal to $70 \mathrm{~V}$ the micromirror appears to be an underdamped system. This matches the conclusion made from the step response tests.

(2) From the phase responses, it can be observed that in low base voltage operation, the micromirror exhibit a larger lag in the frequency range $100 \mathrm{~Hz} \sim 1 \mathrm{KHz}$, than operating in high base voltage. This matches the previous conclusion that the micromirror has a slow response speed when the base voltage is low. 


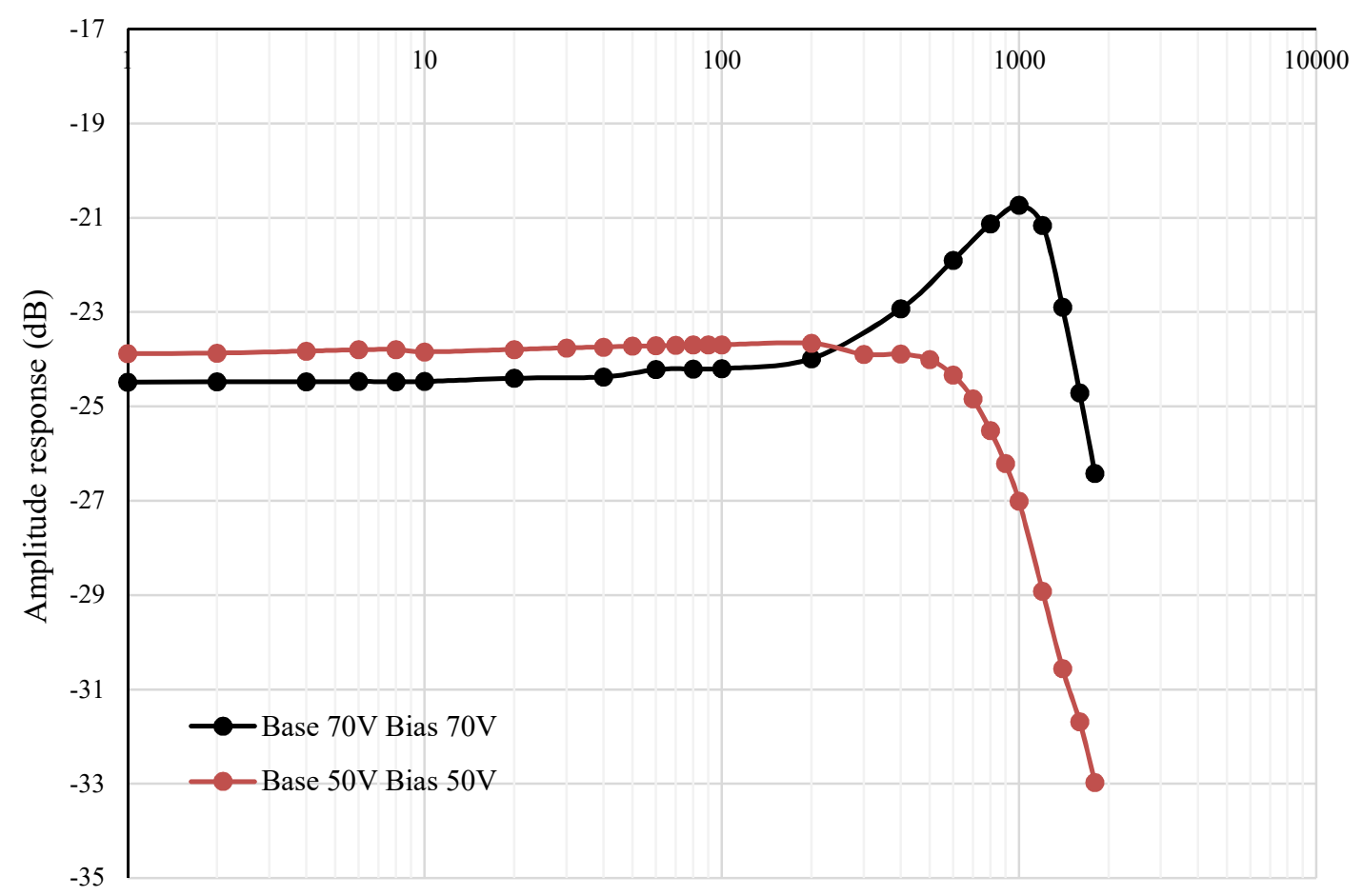

Frequency (Hz)

(a)

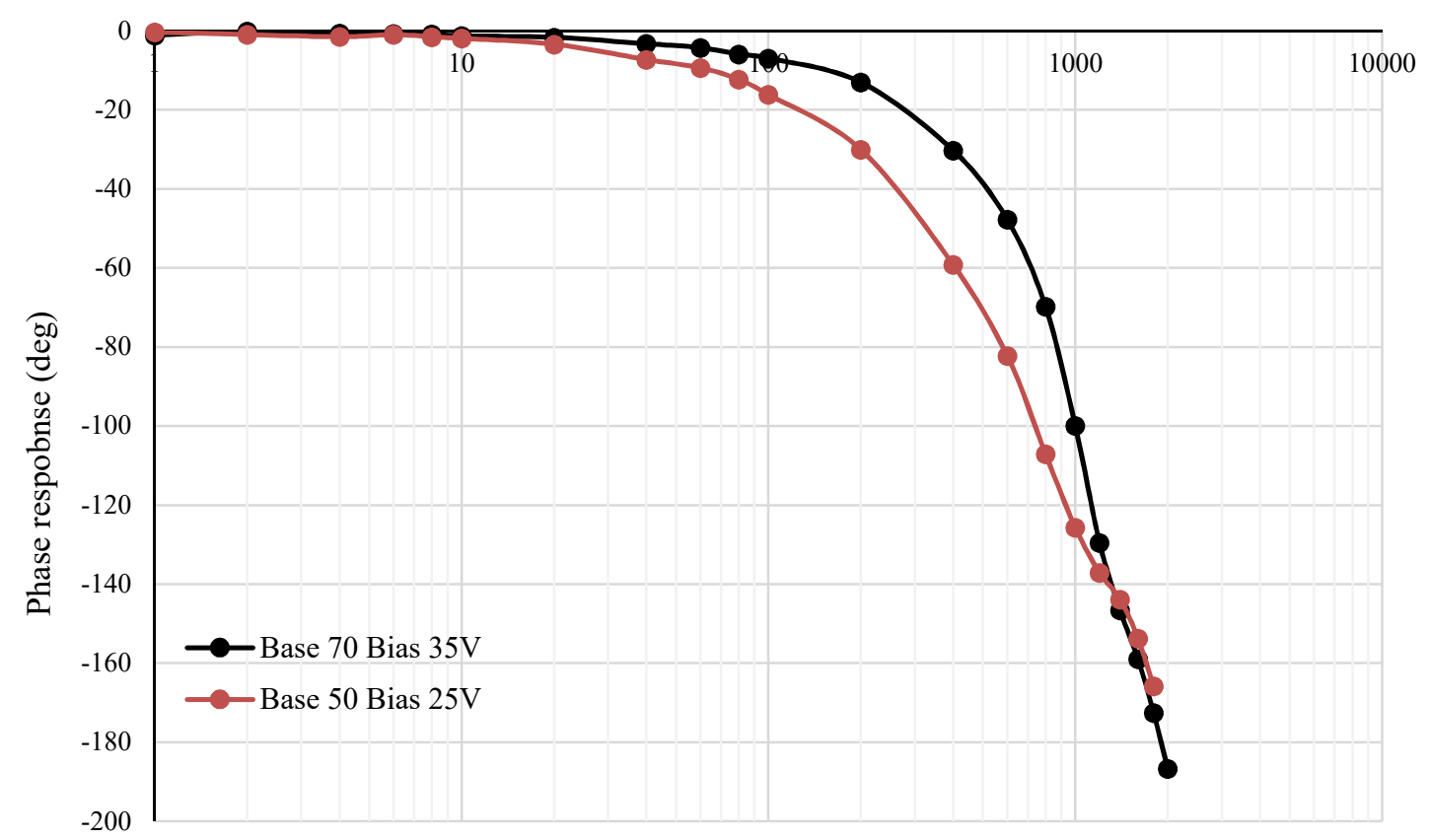

Frequency $(\mathrm{Hz})$

(b)

Fig. 4.16 Bode plot for test 1 and 4 (a) Amplitude response (b) Phase response. 


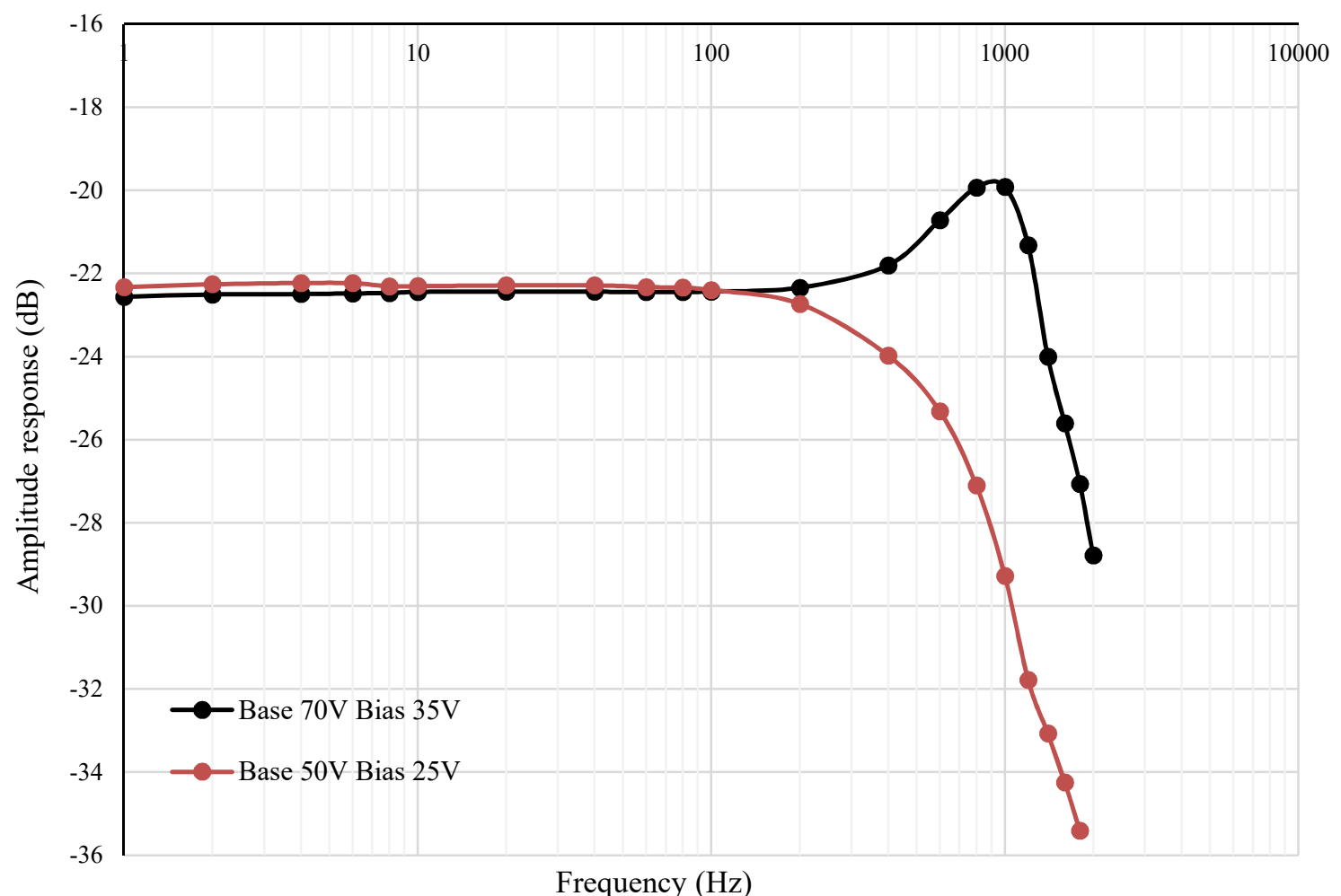

(a)

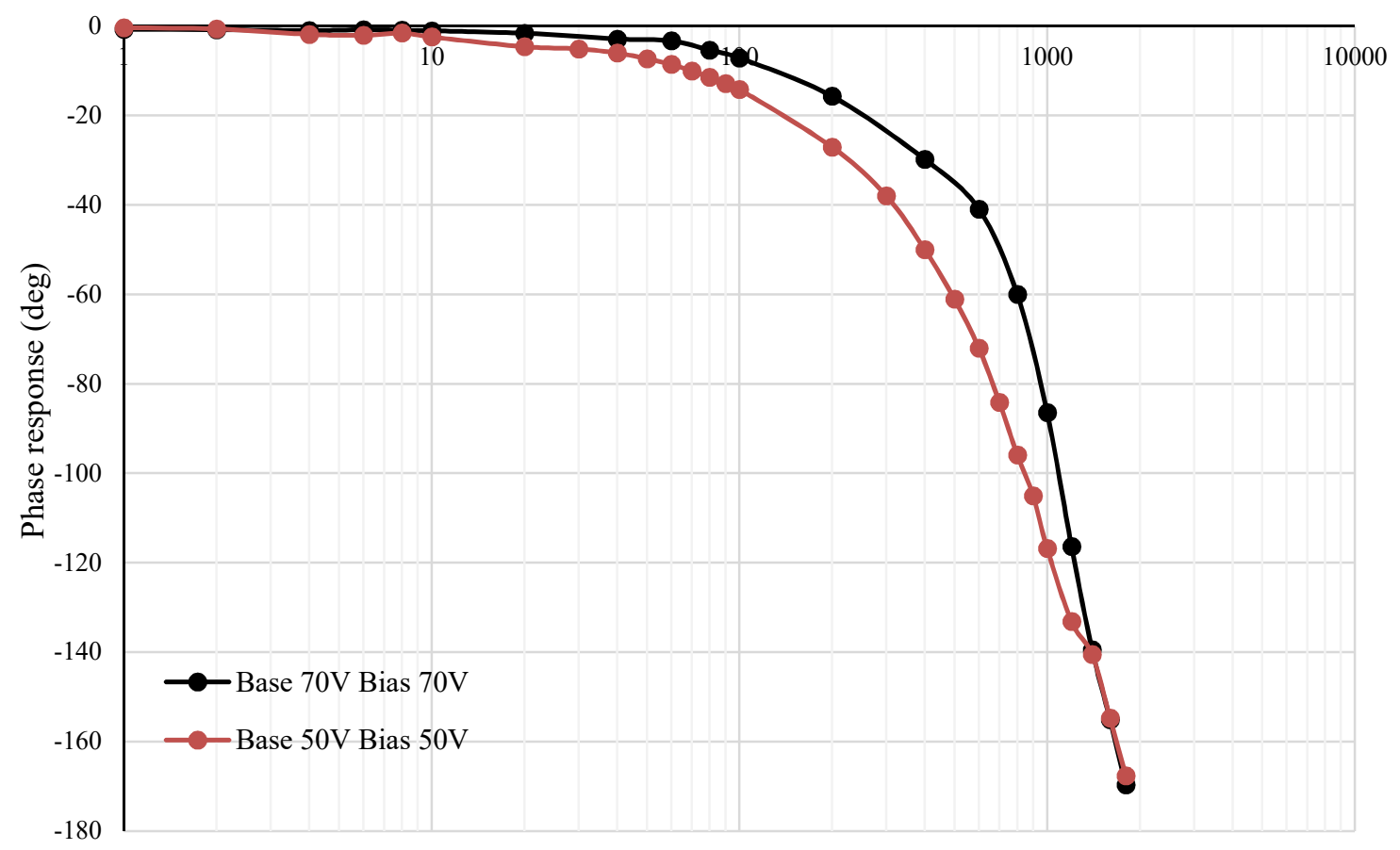

Frequency $(\mathrm{Hz})$

(b)

Fig. 4.17 Bode plot for test 2 and 5 (a) Amplitude response (b) Phase response. 


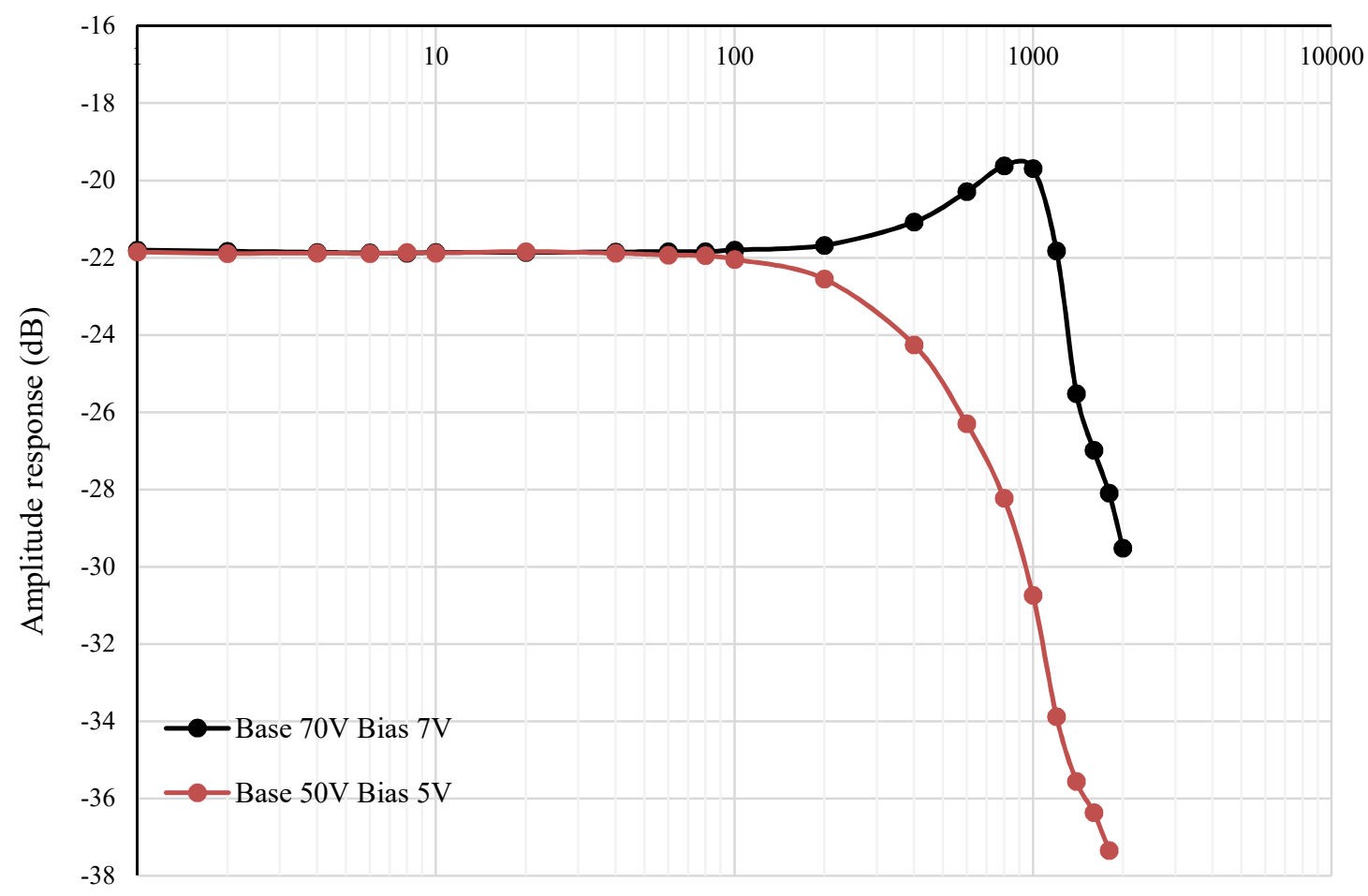

Frequency $(\mathrm{Hz})$

(a)

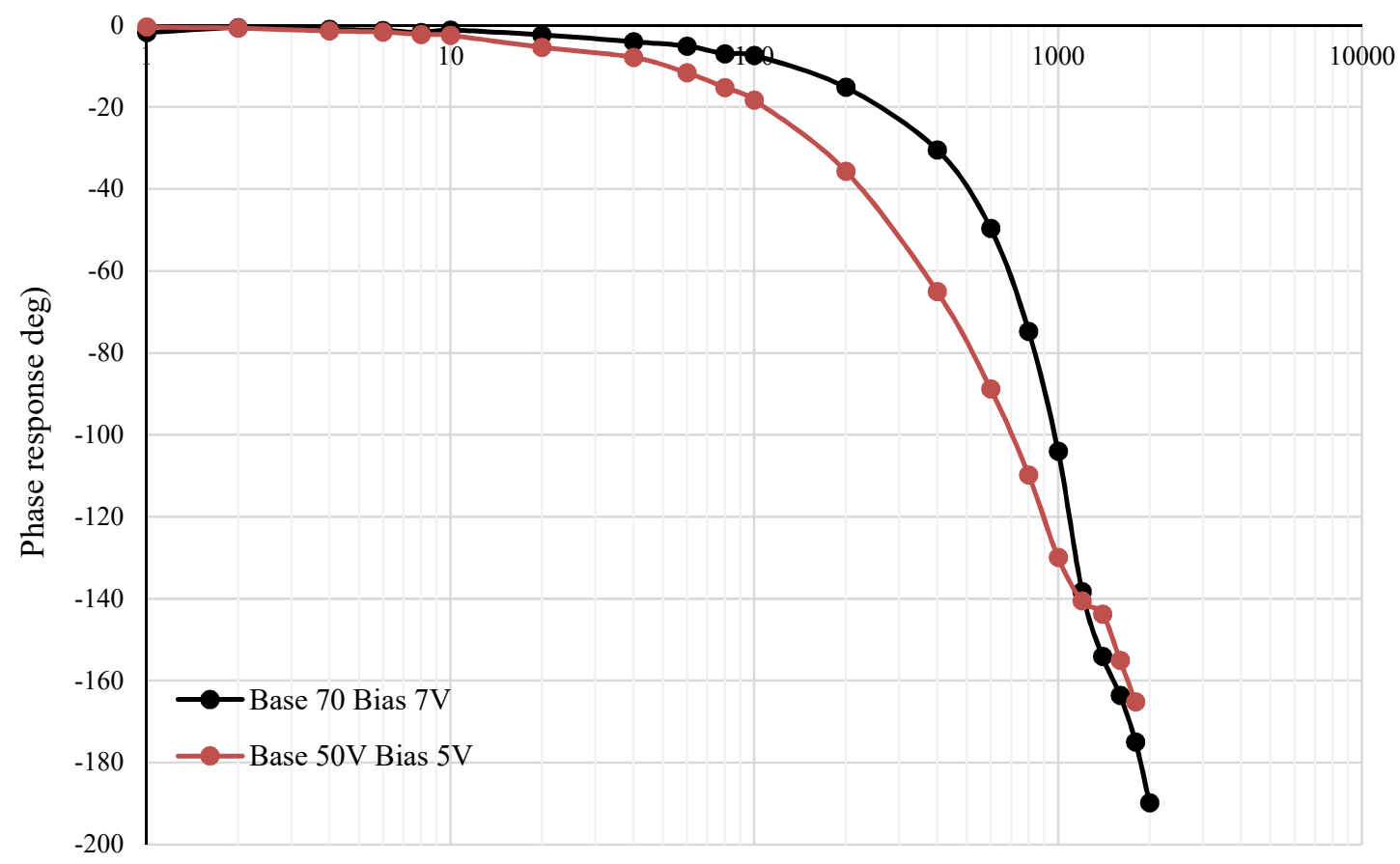

Frequency $(\mathrm{Hz})$

(b)

Fig. 4.18 Bode plot for test 3 and 6 (a) Amplitude response (b) Phase response. 


\subsubsection{Comparison of micromirrors}

The static and dynamic performance of the micromirror presented in this chapter is compared with that of the previously developed micromirror based on ORF actuators. A generic approach is used to control both the micromirrors to display same pattern which is also compared.

\subsubsection{Static performance comparison}

The same tests shown in Table. 4.3 is also performed on the previously developed micromirror based on ORF actuators and the testing results are also shown in Fig. 4.19 (a) together with the static performance of the novel micromirror based on TRF actuator. It can be seen that for various base voltages from $30 \mathrm{~V}$ to $80 \mathrm{~V}$, the presented micromirror have much larger rotation angles than the ORF micromirror. The angles here are the sum of the positive and absolute value of negative rotation angles. The percentage of the improvement is calculated by dividing the rotation angle of the ORF micromirror by the rotation angle difference between the two micromirrors under the same driving voltage. The improvement in percentage is shown in Fig. 4.19 (b) from which it can be concluded that improvement is at least $120 \%$ for various base voltage.

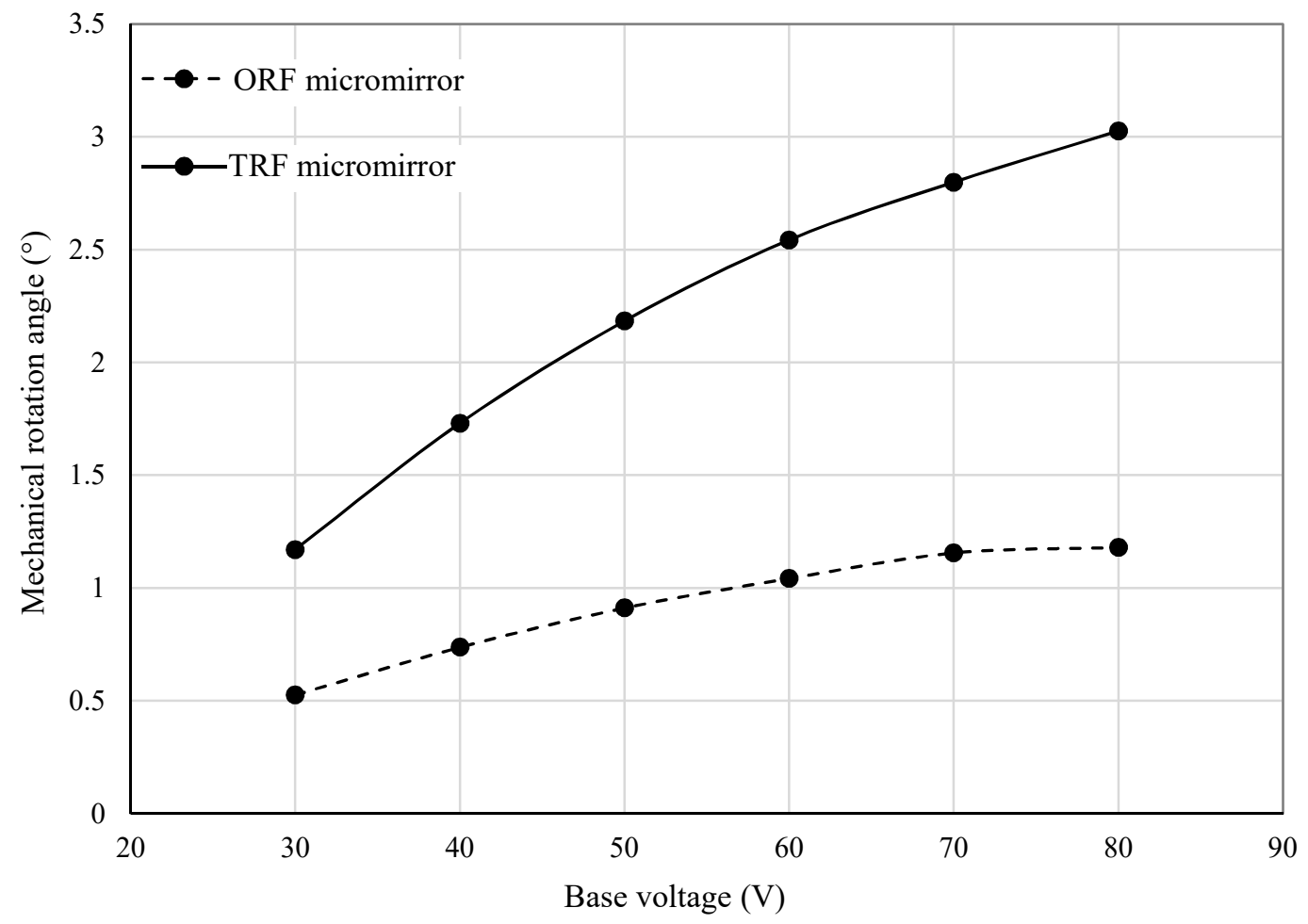

(a) 


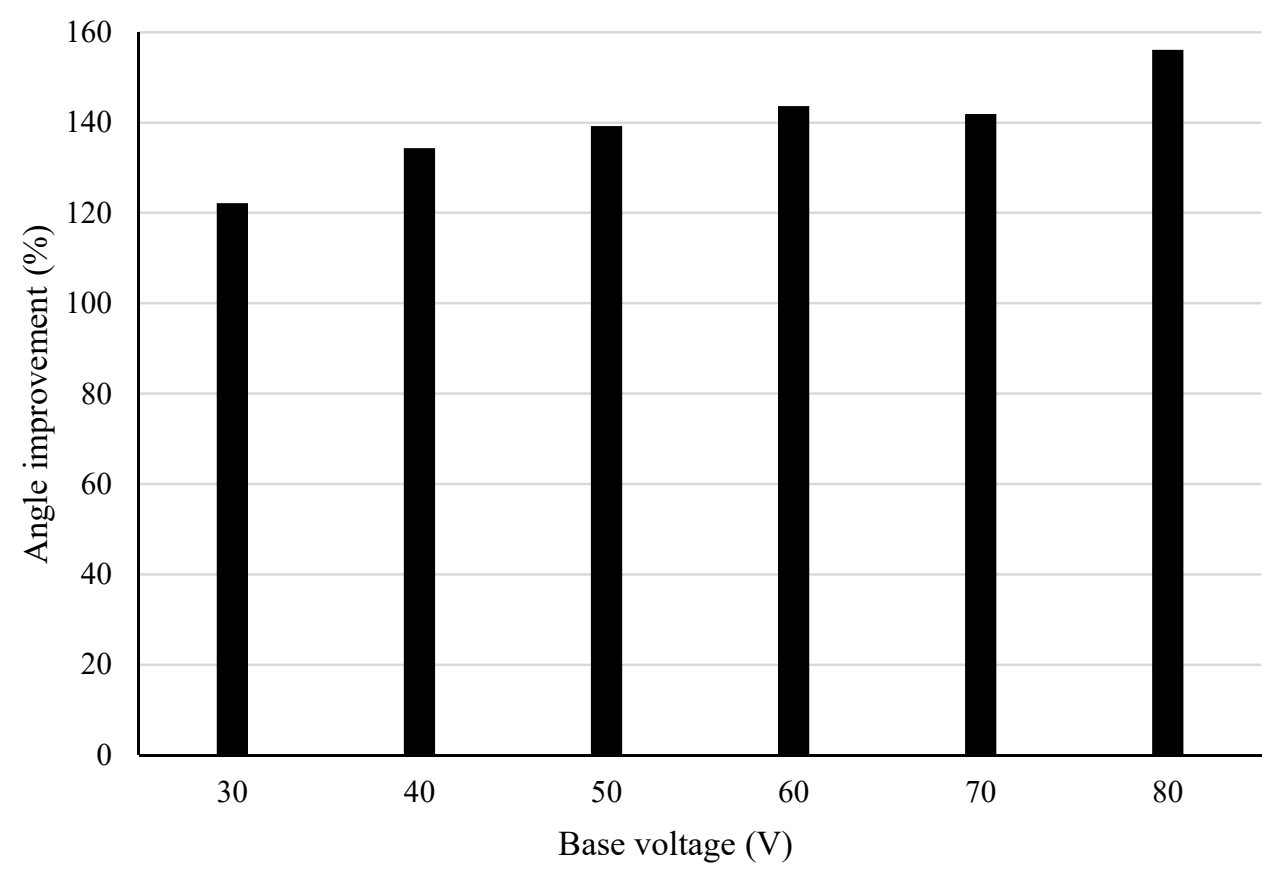

(b)

Fig. 4.19 Micromirror rotation angle comparison.

\subsubsection{Dynamic performance comparison}

The dynamic performance here refers to the response speed of the micromirror. In order to make the comparison, the response speed denoted as $v$ is defined as:

$$
v=\frac{\theta}{T_{r}}
$$

Where $\theta$ is the rotation angle of the micromirror in the step response and $T_{r}$ is the rise time of the step response which is defined as the time from $10 \%$ to $90 \%$ of the total rotation (movement).

The responses of the tests listed in Table. 4.3 are used for comparison and are performed on both micromirrors. The dynamic responses are measured and recorded using PSD. According to Eq. (4.7), two variables $\theta$ and $T_{r}$ are required to obtain $v . \theta$ for both the micromirrors has already been obtained from the above section as shown in Fig. 4.19. The rise time $\left(T_{r}\right)$ can be measured from the recorded dynamic response and are shown in Fig. 4.20. It can be seen that under the same

applied voltage (base and bias voltages), the rise time of the TRF micromirror is lower than that of the ORF micromirror. Considering that the TRF micromirror rotates more than twice of the ORF micromirror while has similar rise times with that of the ORF micromirror, it can be 
concluded that the TRF micromirror has a faster response speed. A quantitative comparison of the response speed is shown in Fig. 4.21 by using Eq. (4.7).

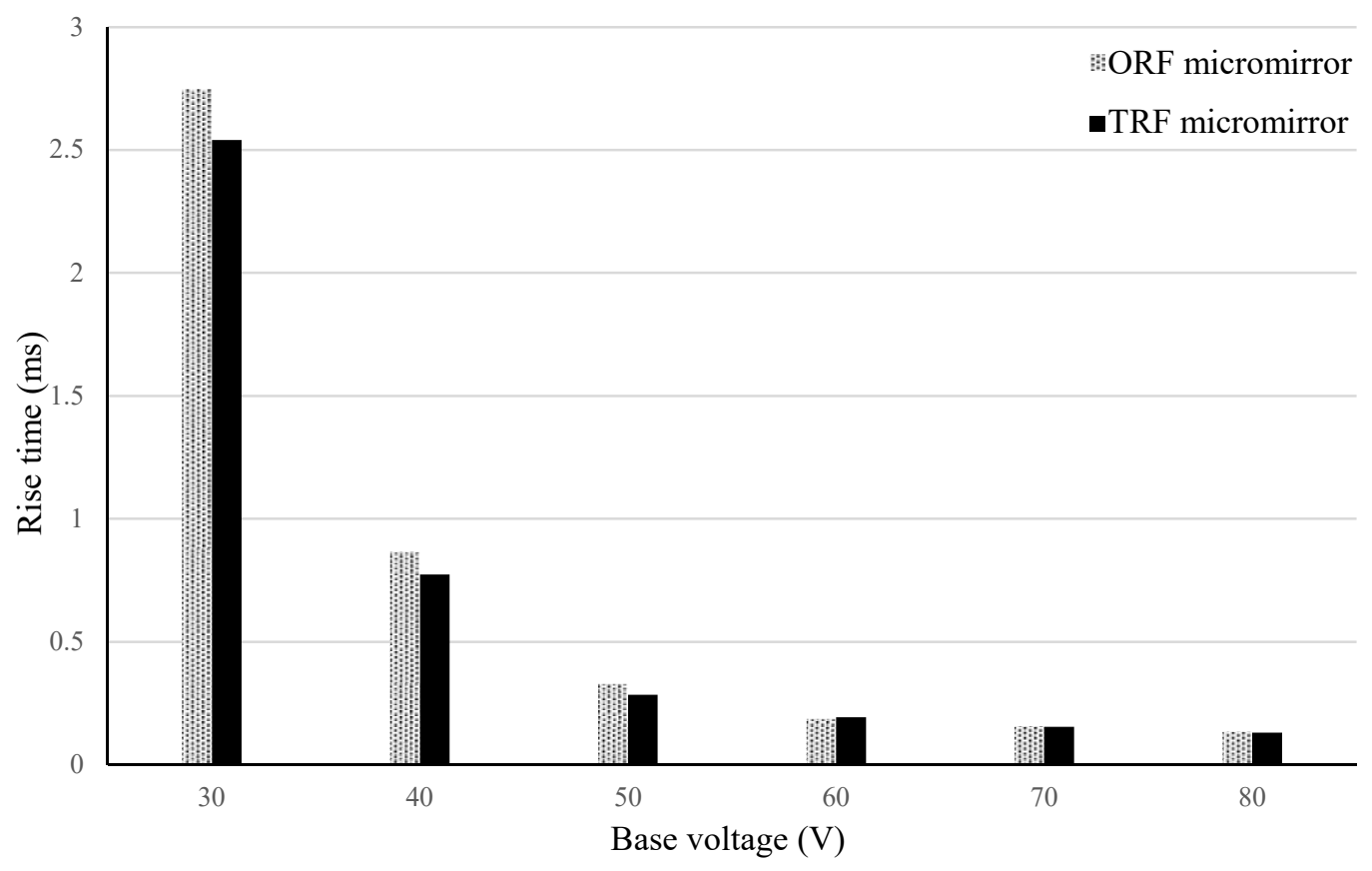

Fig. 4.20 Rise time comparisons of the micromirrors.

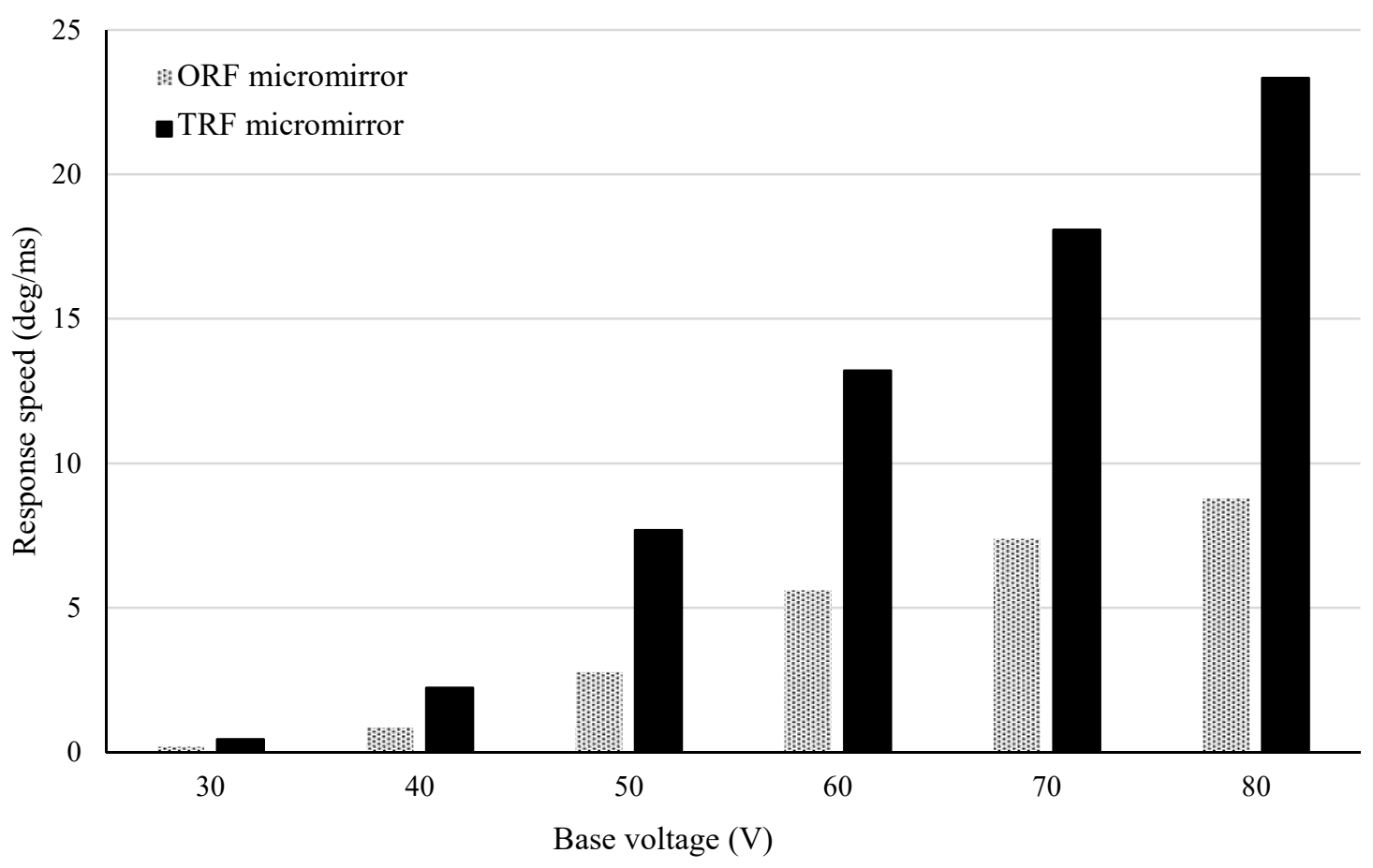

(a) 


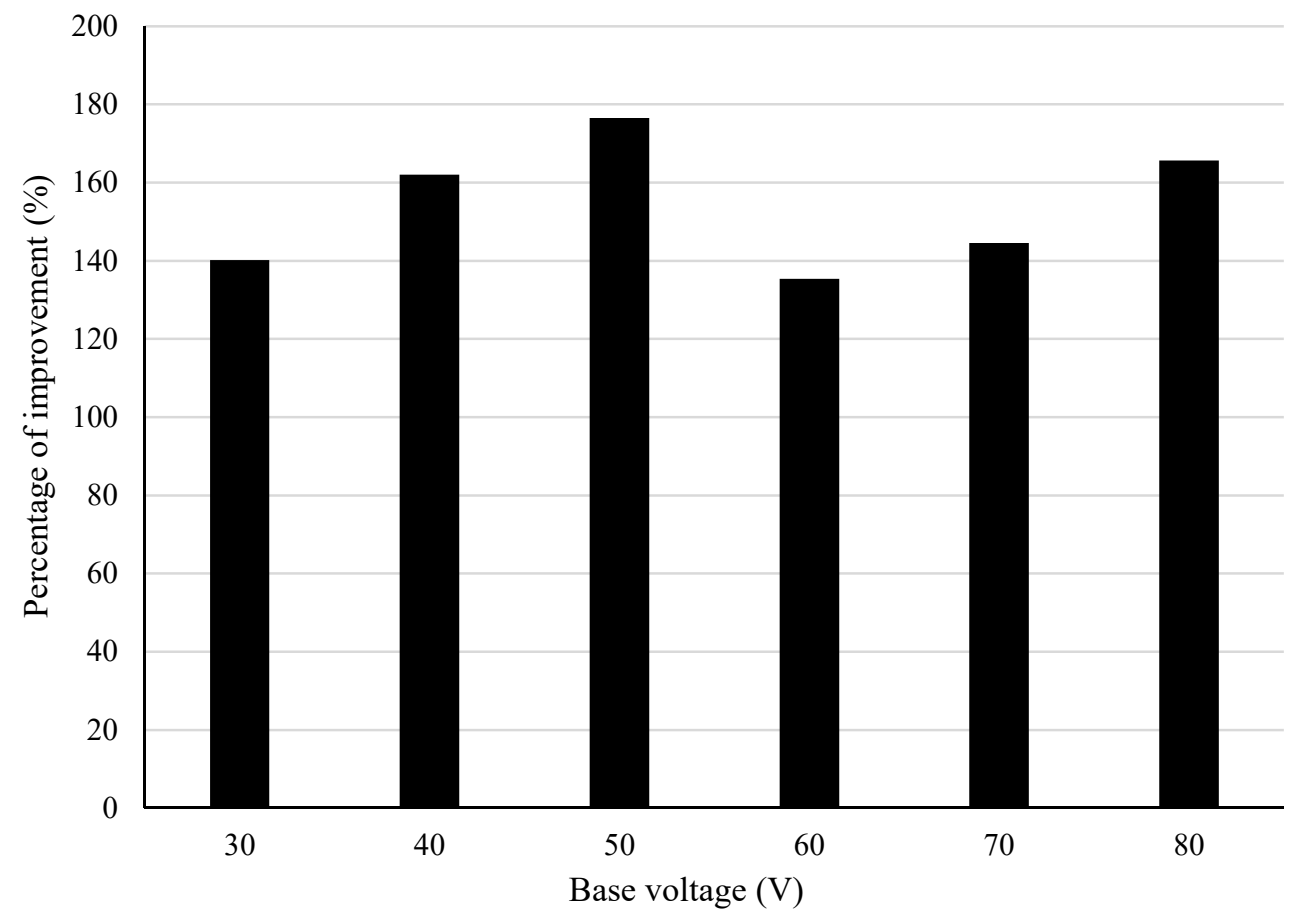

(b)

Fig. 4.21 (a) Response speed compassion of the micromirrors (b) Percentage of the improvement of the response speed of the TRF micromirror compared to the ORF micromirror.

The percentage of the improvement of the response speed is shown in Fig. 4.21 (b). It shows that the response speed of the TRF micromirror is faster than the ORF micromirror under all the base voltage. The improvement is about $135 \% \sim 176 \%$. The maximum and minimum improve happen at the base voltage of $50 \mathrm{~V}$ and $60 \mathrm{~V}$.

\subsubsection{Display result comparison using generic display approach}

The micromirrors presented in this chapter and previously are used to display a same vector pattern which can be potentially used for automotive HUD. The pattern is generated using the generic method presented in [123]. Two display systems are set up on the optical breadboard for two micromirrors separately. The setup of the testing system is similar to the one shown in Fig. 4.7 except that a double side concave lens is added on the reflected laser path to obtain a clear and large display. Two identical double concave lenses are used in two display systems and the distances of the lenses to the micromirrors are adjusted separately to obtain sharp displays on the screens. Due to different curvatures of the two micromirror plates, the distances of the lenses to the micromirrors from which a sharp display can be obtained are different. As a result, the 
magnification factors of the double side concave lenses are different for two display systems. It is measured that the magnification factors are 3.2 and 4.2 for the display system based on the previous and TRF micromirrors respectively. In order to eliminate the effect of different magnification factor of the lens and only compare the scanning range of the micromirrors, the screen is adjusted in the display system of the ORF micromirror so that the distance of the screen to the micromirror is $1.3(4.2 / 3.2)$ times farther than that of the display system of the TRF micromirror. Hence the difference of the magnification factor of two display systems are compensated, and the display sizes of the two display systems can be compared directly.

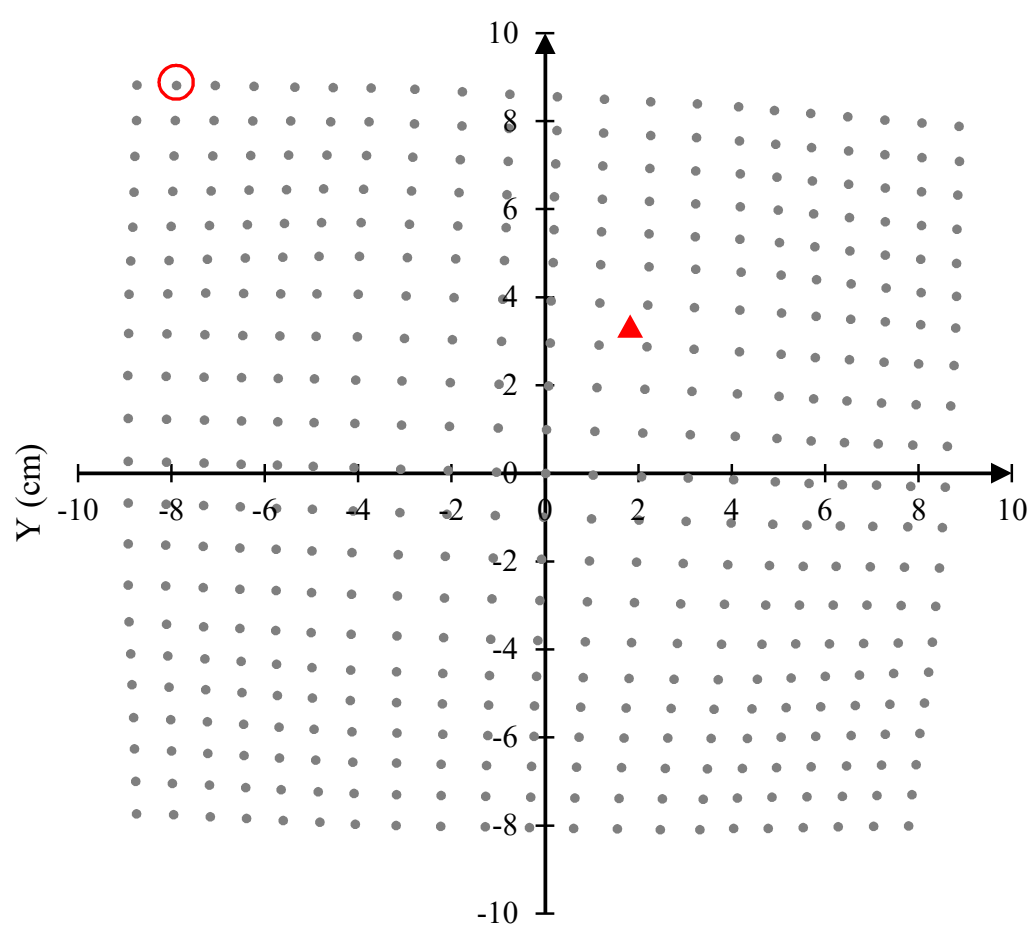

$\mathrm{X}(\mathrm{cm})$

Fig. 4.22 An example of the calibrated points $\left(V_{0}=50 \mathrm{~V}\right)$.

The calibration, which relates the positions of a finite numbers of laser spots on the display screen with their corresponding driving voltages, is performed for both display systems separately. The driving method expressed in Eqs. (4.1) (4.4) are used to generate the calibration voltages. The display system is calibrated by varying the biased voltages, $V_{\text {bias }} a$ and $V_{\text {bias_ }} b$ linearly in their ranges and recording the laser spot locations on the screen. One example of the calibration is shown in Fig. 4.22. For example, the red circled point corresponds to the bias voltages of $V_{\text {bias_a }}=V_{\text {bias } b}$ b $=50 \mathrm{~V}$ with $V_{0}=50 \mathrm{~V}$. For any point on the screen, e.g., the triangle shape point, the corresponding 
bias voltages can be obtained by linearly interpolating the biased voltages of the surrounding four calibrated points. Other than $50 \mathrm{~V}$ base voltage, base voltages such as $60 \mathrm{~V}, 70 \mathrm{~V}, 80 \mathrm{~V}$ are also used to conduct calibration experiments, and the corresponding calibration matrices similar to the one shown in Fig. 4.22 are generated. The higher the based voltage that is used for calibration, the larger the calibration area is.

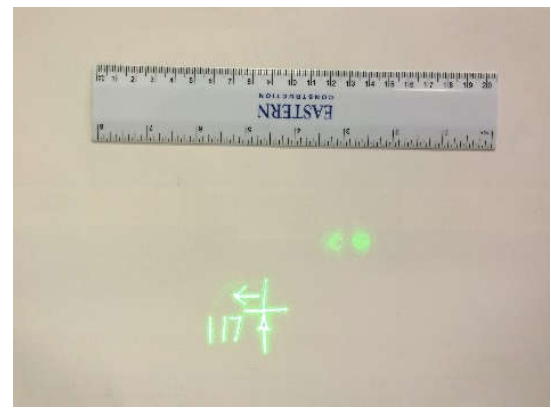

(a)

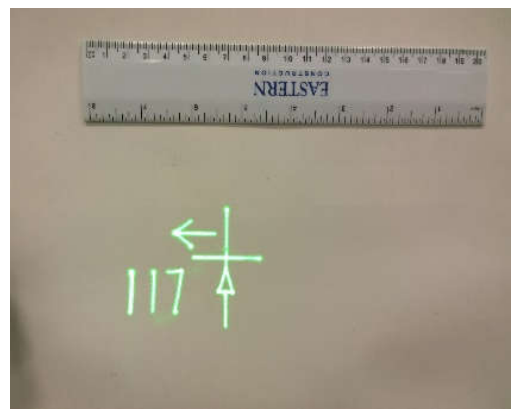

(d)

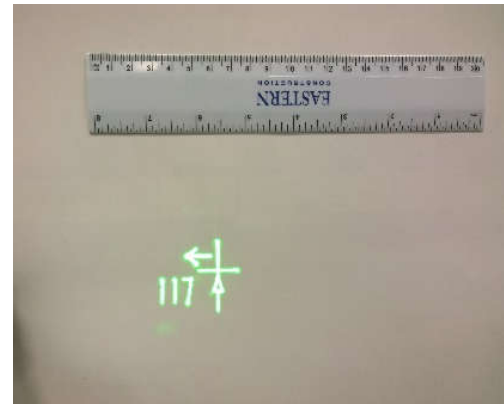

(b)

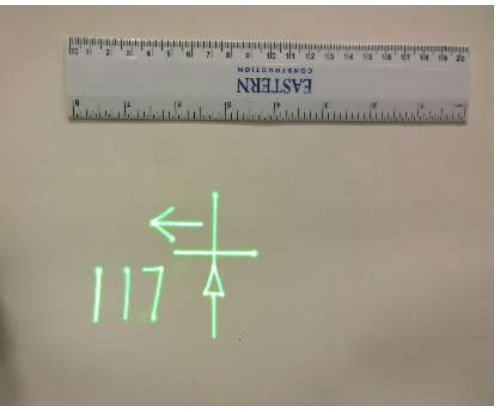

(e)

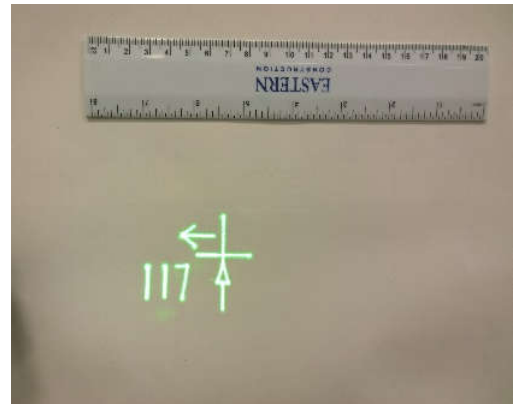

(c)

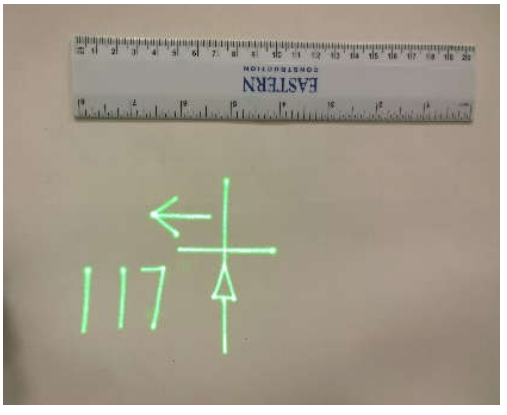

(f)

Fig. 4.23 Display results of the micromirrors (30 Hz) (a) ORF micromirror (b) TRF micromirror display: same size as the display of the ORF micromirror (c) TRF micromirror display: 1.7 times of the size of the ORF micromirror's display (d) TRF micromirror display: 2.8 times of the size of the ORF micromirror's display (e) TRF micromirror display: 4 times of the size of the ORF micromirror's display (f) TRF micromirror display: 5.8 times of the size of the ORF micromirror's display.

The display patterns are designed inside the chosen calibration area, e.g., the one shown in Fig. 4.22. The calibration based on $80 \mathrm{~V}$ base voltage is used to generate the control voltages for the ORF micromirror in order to achieve a relative large display area. The display result is shown in Fig. 4.23 (a) and the maximum voltage applied is about $140 \mathrm{~V}$. In order to obtain a display without severe distortion, the calibration with a relative small base voltage i.e., $60 \mathrm{~V}$, is selected to generate 
the control voltage for the TRF micromirror. By varying the applied voltage, the size of the display of the TRF micromirror changes from small to large as shown in Fig. 4.23 (b) (f). The maximum voltage applied to the micromirror is about $110 \mathrm{~V}$ when displaying the pattern in (f). A ruler is used as a reference to compare the size of displays. It can be seen that even with lower driving voltage, the TRF micromirror still can generate much larger displays. The display distortion increases with the increase of the size of the display. It can be observed from Fig. 4.23 that when the display pattern of the TRF micromirror is about 2.8 times of that of the ORF micromirror, the display quality is still similar. The enlarged views of Fig. 4.23 (a) and (d) are shown in Fig. 4.24.

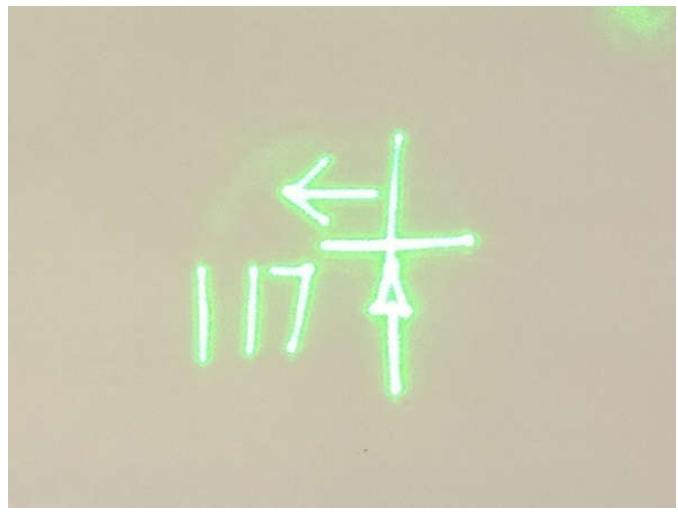

(a)

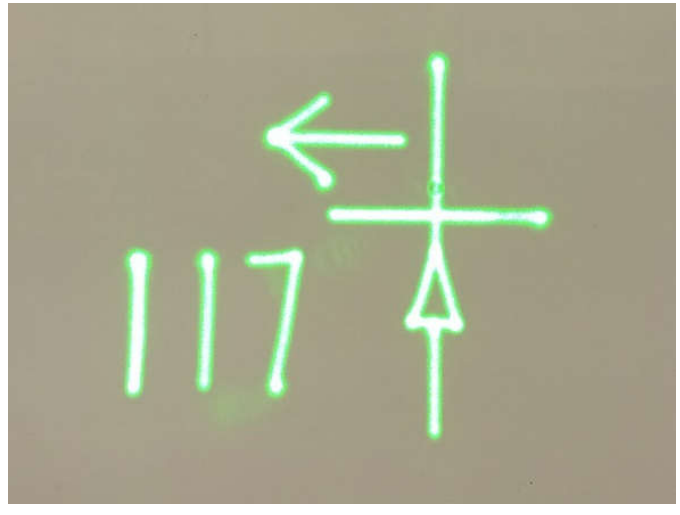

(b)

Fig. 4.24 Enlarged views of the displays in Fig. 4.23 (a) Enlarged view of Fig. 4.23 (a). (b)

$$
\text { Enlarged view of Fig. } 4.23 \text { (d). }
$$

The maximum driving voltage for the TRF micromirror to display the pattern in Fig. 4.23 (d) is $80 \mathrm{~V}$. The maximum driving voltage for the ORF micromirror to display the pattern in Fig. 4.23 (a) is $140 \mathrm{~V}$. It has been found in [107] that the maximum applicable voltage for the ORF micromirror is about $200 \mathrm{~V}$ which can also considered as the upper boundary of the applicable voltage for the TRF micromirror due to the same fabrication process. In this case, the TRF micromirror owing to lower controlling voltage has much larger control capability. Namely, much larger voltage can be applied to the TRF micromirror before it hit the maximum applicable voltage, i.e., there is $120 \mathrm{~V}$ difference from $80 \mathrm{~V}$ to $200 \mathrm{~V}$ compared to that of the ORF micromirror which has only $60 \mathrm{~V}$ difference from $140 \mathrm{~V}$ to $200 \mathrm{~V}$. As a result, the TRF micromirror will have larger voltage range within the safe voltage limit to adjust and increase the response time, which can potentially lead to better display quality. This has been proved in the next chapter that when advance display approach is used, the TRF micromirror can achieve better display quality and 
larger display size than that of the ORF micromirror. The same display of Fig. 4.24 with higher refreshing frequency $(40 \mathrm{~Hz})$ is shown in Fig. 4.25. It can be seen that the display result of the TRF micromirror suffers from less distortion than that of the ORF micromirror. This is attributed to the fact that the TRF micromirror has a faster response speed.
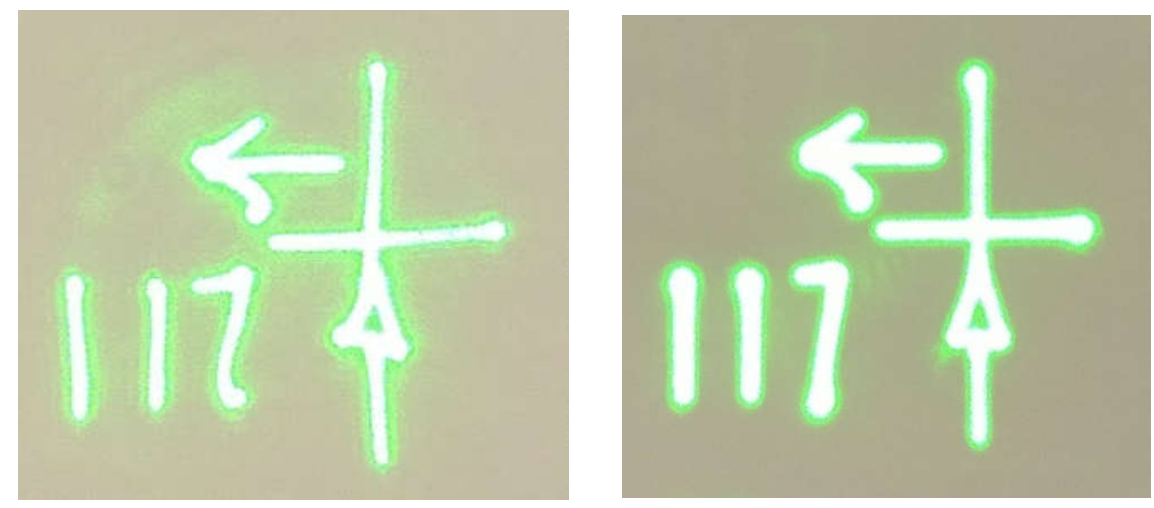

Fig. 4.25 Display results in 40Hz (a) ORF micromirror (b) TRF micromirror

The display pattern presented in [109], [123] are also displayed using both micromirrors. The display results are shown in Fig. 4.26. Smaller driving voltages are applied to the TRF micromirror to generate a larger pattern than that of the ORF micromirror. The display quality of the TRF micromirror is slightly improved compared to that of the ORF one.

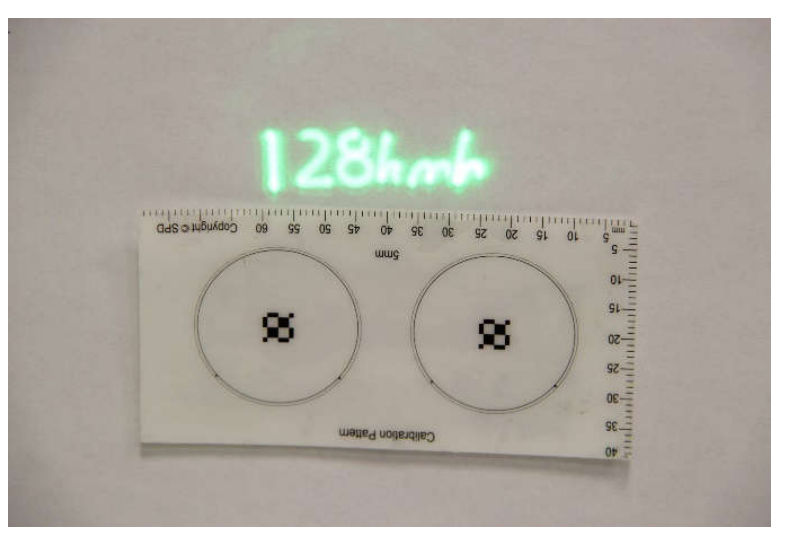

(a)

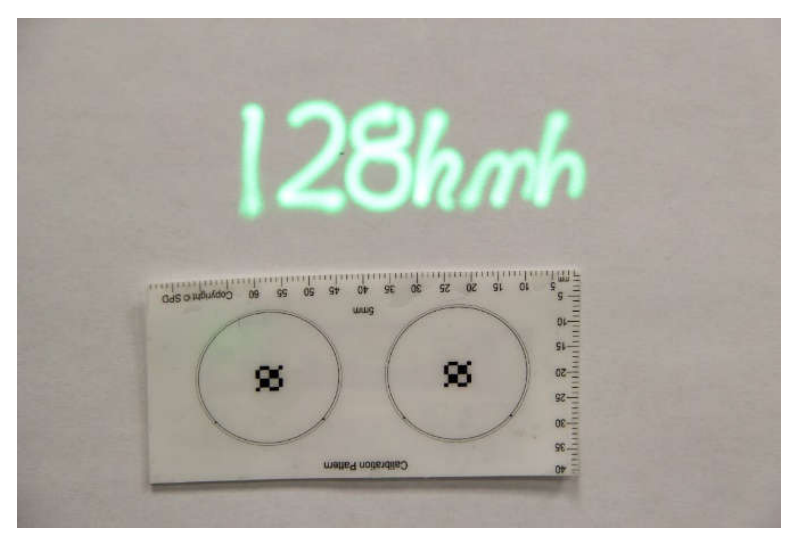

(b)

Fig. 4.26 Display results of ORF and TRF micromirror in $40 \mathrm{~Hz}$ (a) ORF micromirror (b) TRF micromirror.

\subsection{Summary}

From the above comparisons, it can be concluded that the TRF micromirror can display much larger vector graphics with smaller driving voltage. The TRF micromirror is able to generate vector 
patterns with less distortion than that of the ORF micromirror due to faster response speed. More important, the lower driving voltage of the TRF micromirror provides it with much larger control capability than the ORF micromirror. This enables the display result of the TRF micromirror to be greatly improved by developing proper control algorithm. As a result, an advanced display approach will be presented in the next chapter which can significantly improve the display capability of the TRF micromirror. 


\section{Chapter 5 Advanced display approach for micromirror based vector display}

This chapter is based on the following paper that is to be submitted:

Chao Fan and Siyuan He, "Micromirror based automotive head up display (HUD)", IEEE Transaction on Mechatronics.

In this chapter, an advanced display approach for micromirror based vector display is presented. This approach consists of the following steps: 1) Display system calibration; 2) Scanning trajectory design; 3) Determination of point number for each segment; 4) Allocate display points for each segment; 5) Assign voltages to points. All above steps are presented in this chapter. The comparison between the advanced display approach and the previously developed generic display approach is also presented.

\subsection{Criteria of display quality}

The display quality is important for a HUD system, the quality of the image is defined by 4 criteria, i.e., 1) refreshing rate, 2) distortion and 3) brightness of the display 4) the uniformity of the illuminance. Refreshing rate is the number of times that the laser beam scans through a designed trajectory in one second in the unit of Hz. Due to the persistence of vision theory, in order for a person to perceive a stable display, a large refreshing rate is always desired for a display system, i.e., equal or greater than $30 \mathrm{~Hz}$. Distortion is the observable deviation of the scanning laser path from the desired trajectory. Brightness of the image is another important criterion for the display system which indicates how much luminous power of the displayed patterns can be observed in a certain angle, it has the unit of $\mathrm{cd} / \mathrm{m}$. The uniformity of the illuminance indicate how uniform the light is illuminated from the display.

The degree of distortion is positive correlated with the refreshing rate. Namely, a larger frame frequency will lead to a more severe distorted display. Also it is related to the dynamic response of the micromirror. Some display distortions are illustrated in Fig. 5.1. It is assumed that the laser trajectory is composed with two line segments which has $90^{\circ}$ between each other. The scanning laser beam is controlled to follow 3 vertexes, $\mathrm{A}, \mathrm{B}$ and $\mathrm{C}$ which are labeled in Fig. 5.1. The 
distortion shown in Fig. 5.1 (a) is caused due to the fact that the command to drive the laser beam to move to $\mathrm{C}$ is sent out before it reaches point $\mathrm{B}$. As a result, the right angle vertex is distorted into a curve. This kind of distortion can be improved by accelerating the response speed of the micromirror. Another distortion is shown in Fig. 5.1 (b) which is less common than the first type of the distortion. This distortion is caused only by the overshoot of the system, the laser beam goes beyond the point B first and then stabilized at B after which it proceeds to point $\mathrm{C}$. This situation happens when the system has the overshoot and enough time is given to the scanning. As a result, the laser beam will first be stabilized at B before it proceeds to C. However, in most cases in order to achieve a desired refreshing rate, the time provided for the scanning is not enough for it to be fully settled down before the BC driving command is given. Instead, the distortion in Fig. 5.1 (c) is most likely to happen in the display. It is caused by both overshoot and the slow response speed. In this case, a much more severe distortion is generated compared to that in (a) and (b).

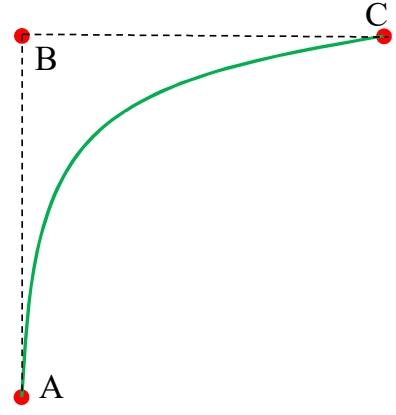

(a)

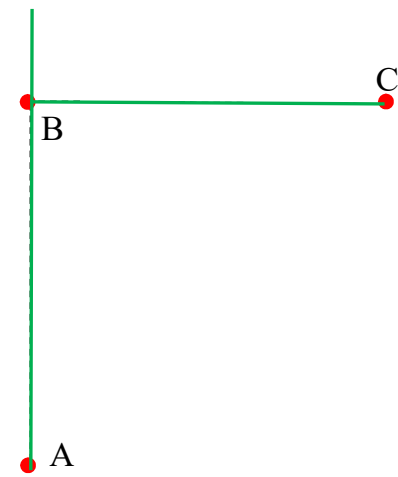

(b)

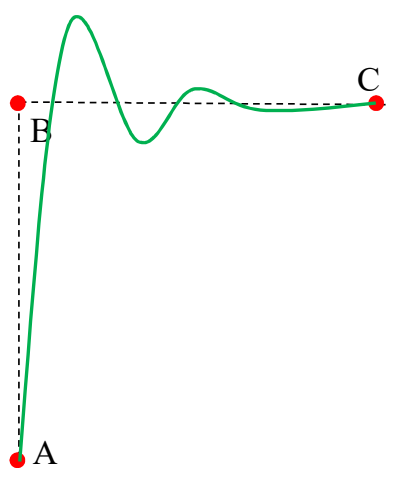

(c)

Fig. 5.1 Some simple examples of display distortion.

To obtain a display with good quality, the undesired dynamic behavior of the micromirror such as large overshoot and slow response speed need to be overcome. Open loop and close loop control are the two control schemes that are usually used for the micro-device control. Each scheme has its own advantages and disadvantages [144]. Open loop control uses the pre-designed signal to drive the micromirror directly which does not require a high computational controller. So the open loop controlled system has much less calculation burden, and can be implemented by simple microprocessor, which will lead to low cost and compact devices. Also, the close loop control of the presented display system requires sensing mechanism. The high speed scanning requires optical sensors, i.e., position sensing detector (PSD) with high speed. The PSD with high sampling 
rate $(>1 \mathrm{MHz})$ and relative large sensing area lead to a system with much higher cost than open loop controlled system. As a result, an open loop control mechanism is developed to control the micromirror in this section.

An effective open loop control approach of the micromirror to track a trajectory is to drive the micromirror to have a point to point motion through a number of properly distributed points on the trajectory. Due to the dynamic performance of the micromirror such as large overshoot and slow response speed, the displayed pattern will be distorted. The undesired distortion can be reduced or even eliminated by properly designing the distribution of the points.

The proposed advanced display approach of generating the control points is composed of 5 steps. The $1^{\text {st }}$ step is to calibrate the display system to obtain the relationship between the driving voltages of the micromirror with the locations of the corresponding laser spots on the display screen. The $2^{\text {st }}$ step is to design the scanning trajectory inside the calibration area. The trajectory is designed to be only composed of line segment, and the end points (critical points) for all the segments are obtained. The $3^{\text {rd }}$ step is to assign the number of control points to each line segment. The $4^{\text {th }}$ step is to design the distribution of the control points on the line segments. The last step is to optimize the design parameters to obtain good display quality using trial and error method. The detailed approach are discussed as follows.

\subsection{Display system calibration}

Calibration of the display system is a characterization procedure which relates the positions of a series of points on the display screen with their corresponding driving voltages. The relation between the driving voltages and the locations of the corresponding dots on the display includes both the electromechanical coupling and optical refraction. Electromechanical coupling is the relationship between the applied voltages and the corresponding rotation angles of the micromirror. The optical coupling refers to the effect of the double side concave lens on the scanning angles (amplification and distortion). The calibration is an easy way to accurately capture all these complicated relations.

Position Sensing Detector (PSD) is usually used to detect the laser spot position. The output of a PSD provides us with the coordinates of the centroid of the incident light spot. A PSD based calibration approach has been presented in [109], [123]. However, due to the rough surface of the surface machined micromirror plate and the protective glass of the micromirror, the reflected laser 
spot is usually noisy and the PSD is likely to fail to provide the accurate position of the laser spots. Ideally because the incident laser beam is a circle in shape, the reflected spot on the sensor should also be approximately a circle; however, the actual reflected laser beam is usually a complicated patterns shown in Fig. 5.2. In Fig. 5.2 the reflected laser beam has one big center circle and some small laser dots around. This is due to the fact that the micromirror is fabricated by surface micromachining process which leads to releasing holes and dimples on its surface. This will cause a poor surface quality. The small features on the micromirror plate are reflected to the display together with the desired laser spot. Some of the noisy dots moves with the micromirror while some does not and the intensity of noisy dots changes with the movement of the micromirror. Also, there is always a protective anti-glare glass covering the micromirror. Even the glass is anti-glare there is still a visible dot reflected to the screen which does not move as the micromirror moves. Since PSD provides the coordinate of the laser spot by calculating the centroid of all the light illuminating on it, the time changing intensity of the illuminance caused by the noisy light will have a significant effect on the accuracy of the PSD. As a result, it is not the best choice for the calibration of the micromirror based scanning display system. In order to improve the accuracy of the calibration, a camera based calibration method is developed in this section which uses a camera to capture the calibrated points on the display screen. The noisy light can be eliminated using image processing methods and a more accurate result can be obtained.

In order to perform calibration, a set of control voltage combinations are generated using Eqs. (4.1) (4.4) by linearly changing the two bias voltages. The generated voltage combinations are then applied to the micromirror one combination by one combination. The positions of each laser spot on the 2D display plane together with their driving voltages are captured by the camera. The system setup of the calibration is shown in Fig. 5.2 and the reflected laser beam scans a spot matrix which is shown in Fig. 5.3.

A DSLR camera due to its high image resolution, variable focal length and long exposure time is used to capture the reflected laser spots in one shot for all the voltage combinations applied to the micromirror. The camera is positioned in front of the display screen and the focal length of the camera lens is adjusted so that the camera is focused on the laser spots, and the laser spot matrix lies in the center of the view. The predefined driving voltage combinations are applied to the micromirror in a certain sequence and there is a hold time between each combination of the driving voltage, which gives enough time for the micromirror plate to be fully stabilized and stays in the 
steady position for a short time period. The laser is modulated to be only turned on during the period that the micromirror is stabilized for each driving voltage set.

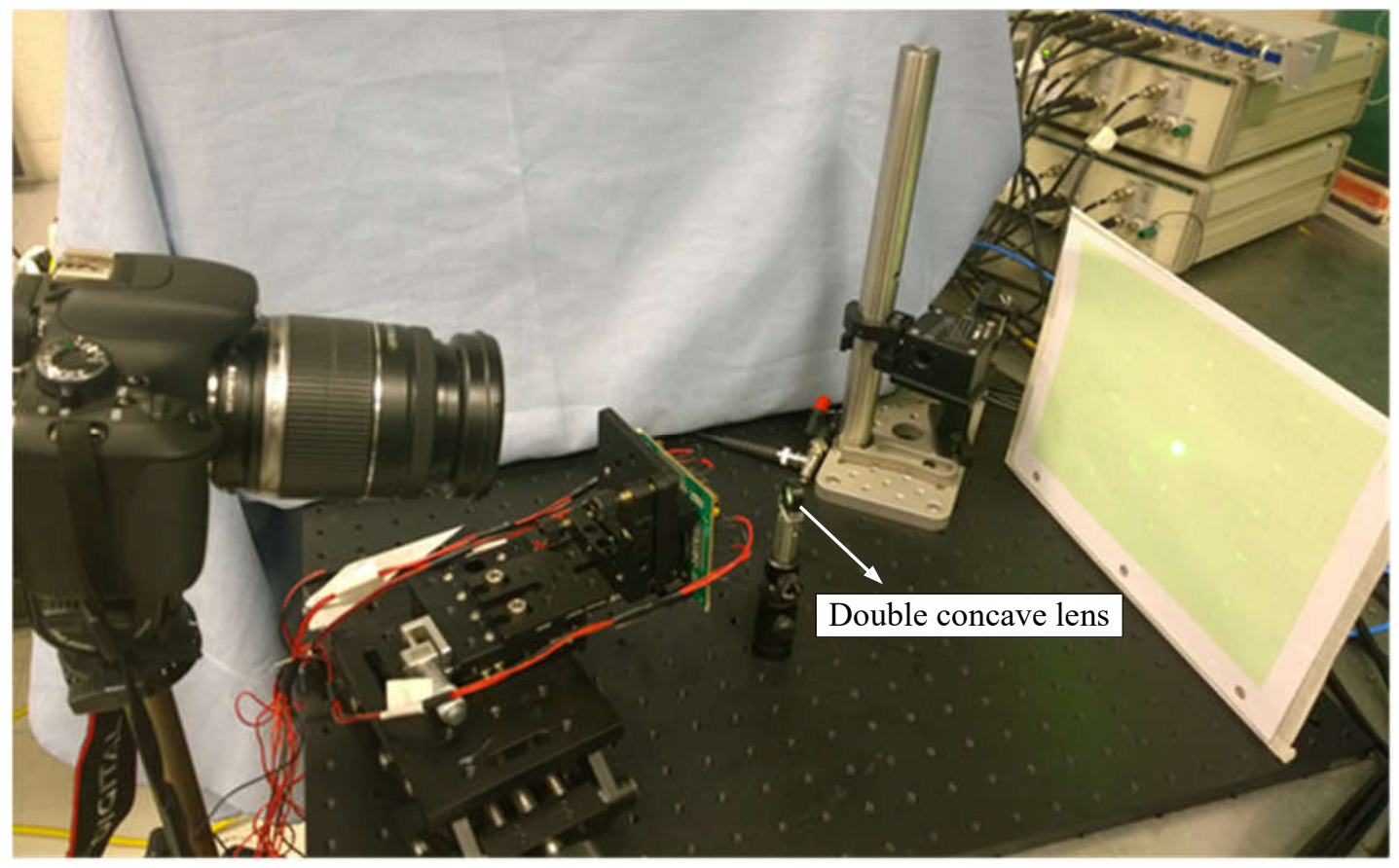

Fig. 5.2 Calibration system setup.

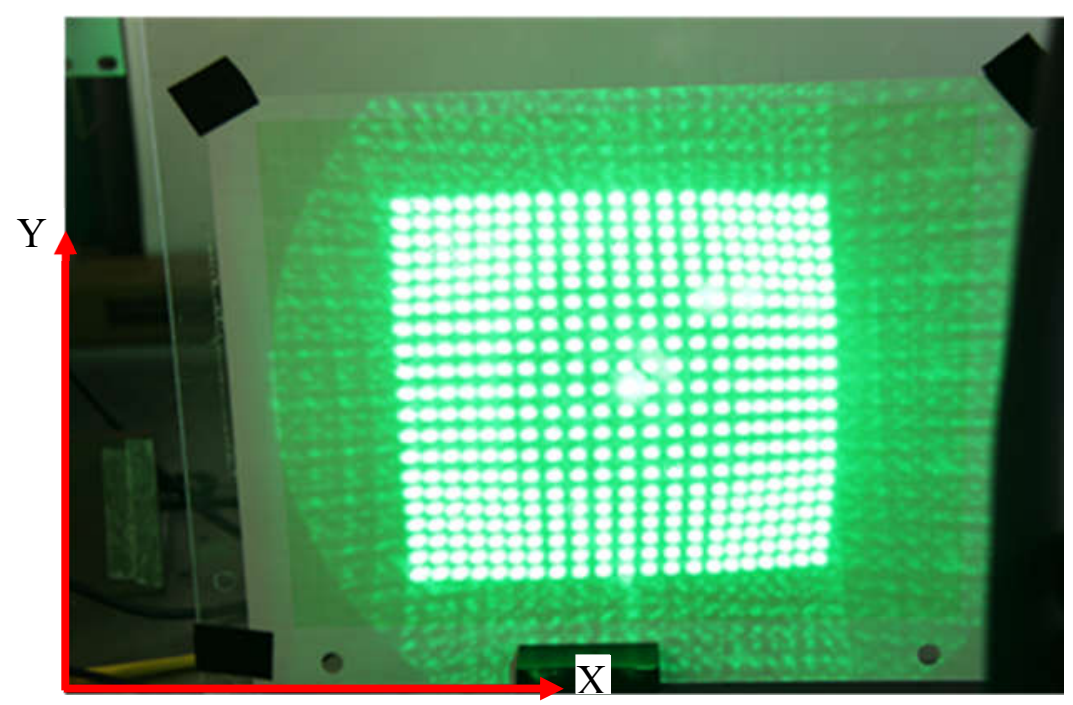

Fig. 5.3 Calibration matrix.

Cannon EOS Rebel T3i with $18-200 \mathrm{~mm}$ focal lens is used for the calibration. The exposure time is set to be its maximum which is 30 s so that as many points can be calibrated. The number of the driving voltage combinations needs to be carefully decided. If the number is too large, it will lead to a dense laser spot matrix from which the adjacent spots are hard to be distinguished 
by image processing. While a too small number will have few calibration spots which will lead to a low resolution calibration model. In practice, the number is decided by trial and error method and a total number of 441 driving voltage sets are used to generate a $21 \times 21$ laser spot matrix. The hold time between voltage sets is set be $65 \mathrm{~ms}$ and the on time for the laser is set to be $15 \mathrm{~ms}$ which leaves $50 \mathrm{~ms}$ for the micromirror to reach steady state. Due to the long exposure time, in order to get a properly exposed image, the light illumination of the background needs to be controlled at a low level. The recorded image is shown in Fig. 5.3.

Image processing method is used to extract the coordinate of each laser spot in the image coordinate system that is shown in Fig. 5.3. Image processing and analysis software ImageJ is used to process the image. It can be observed from Fig. 5.3 that due to the roughness of the micromirror plate a lot of green light noise has been introduced and enhanced by long exposure time. An RGB image can be split into 3 independent images each one contain only one color component and the brightness of each pixel is measured by the grey scale ranging from 0 to 255 . By observation, the red channel image has the least green light noise while the spot matrix still keeps a good contrast with the background as shown in Fig. 5.4. It can also be observed that there are some spots still connected which will be hard to be separated simply by using the image processing. In this case, free hand modification is needed to manually separate a few connected points as shown in Fig. 5.5. After the modification, the threshold of the image is adjusted and converted into binary image (black and white) as shown in Fig. 5.6. Then the "fillhole", "close", "open", and noise canceling operation are performed on the image to make all the spot area disconnected with a relative smooth edge. The final image after processing is shown in Fig. 5.7.

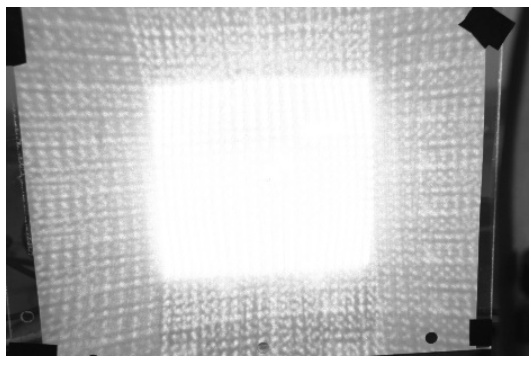

(a)

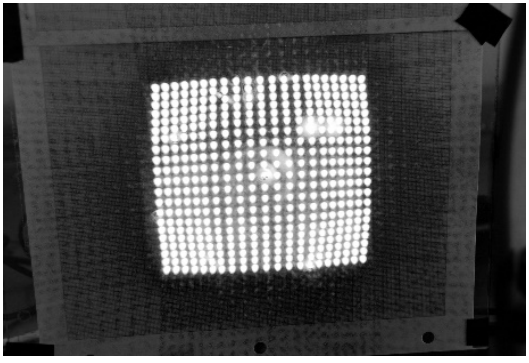

(b)

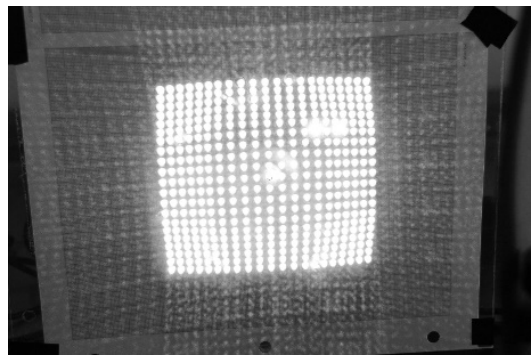

(c)

Fig. 5.4 Calibration RGB image split by 3 color channels (a) Green Channel image (b) Red channel image (c) Blue channel image. 


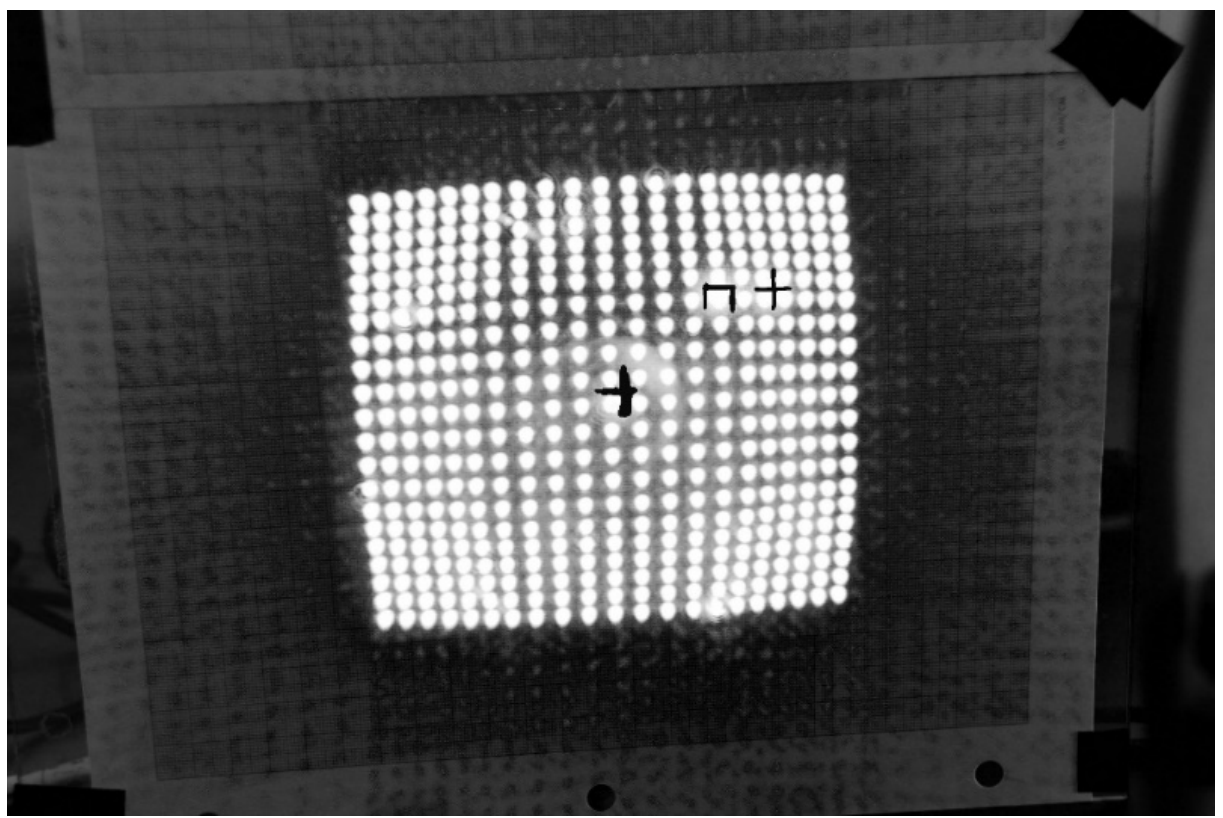

Fig. 5.5 Modified red channel image.

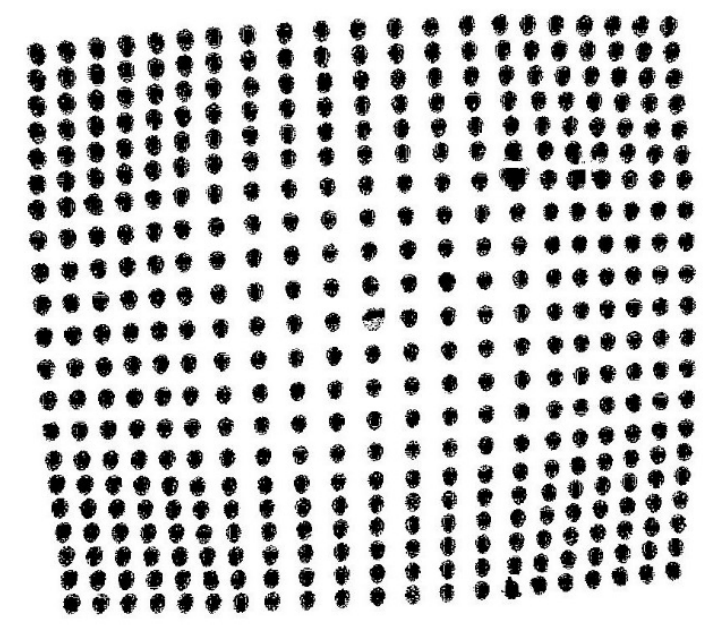

Fig. 5.6 Binary image converted from the modified red channel image.

Then the coordinate of the center of each spot on the image can be extracted by the particle analysis function of the ImageJ. A look-up table can be built by mapping the coordinates of all of the calibrated points to their corresponding driving voltages. The driving voltages of any noncalibrated point with known image coordinates can be obtained by interpolating the driving voltages of the surrounding 4 points. 


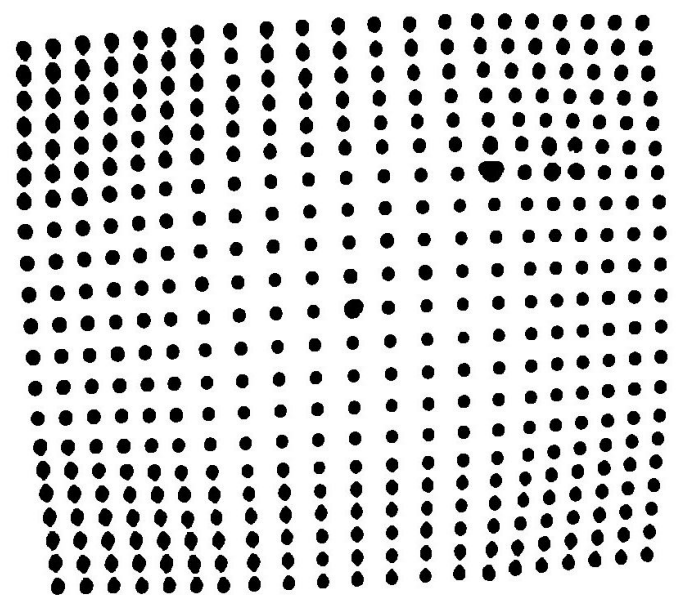

Fig. 5.7 Binary image after smooth operations.

\subsection{Scanning trajectory design}

After the calibration is performed as presented in the above section, vector graphics need to be designed inside the calibration area. One example is shown in Fig. 5.8.

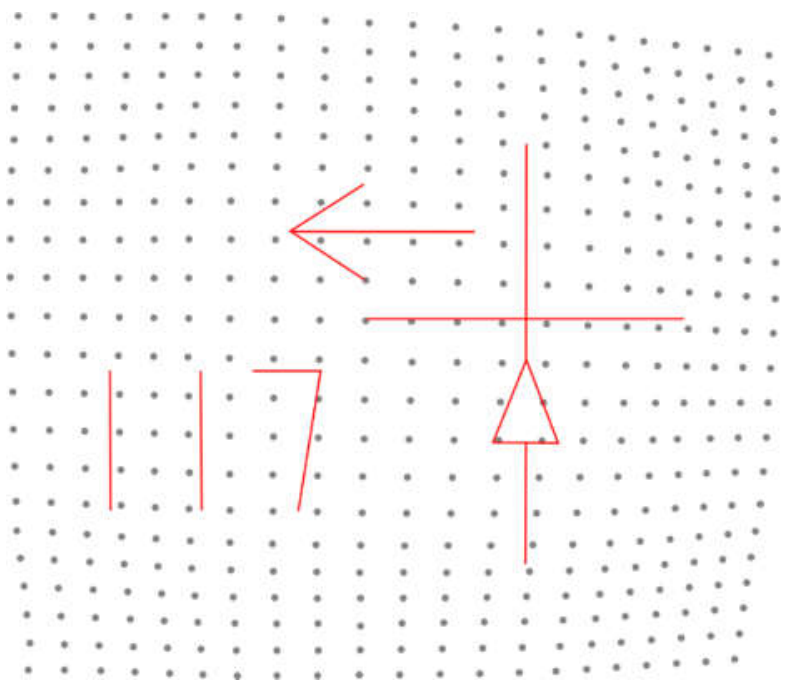

Fig. 5.8 Designed trajectory inside the calibration area. 

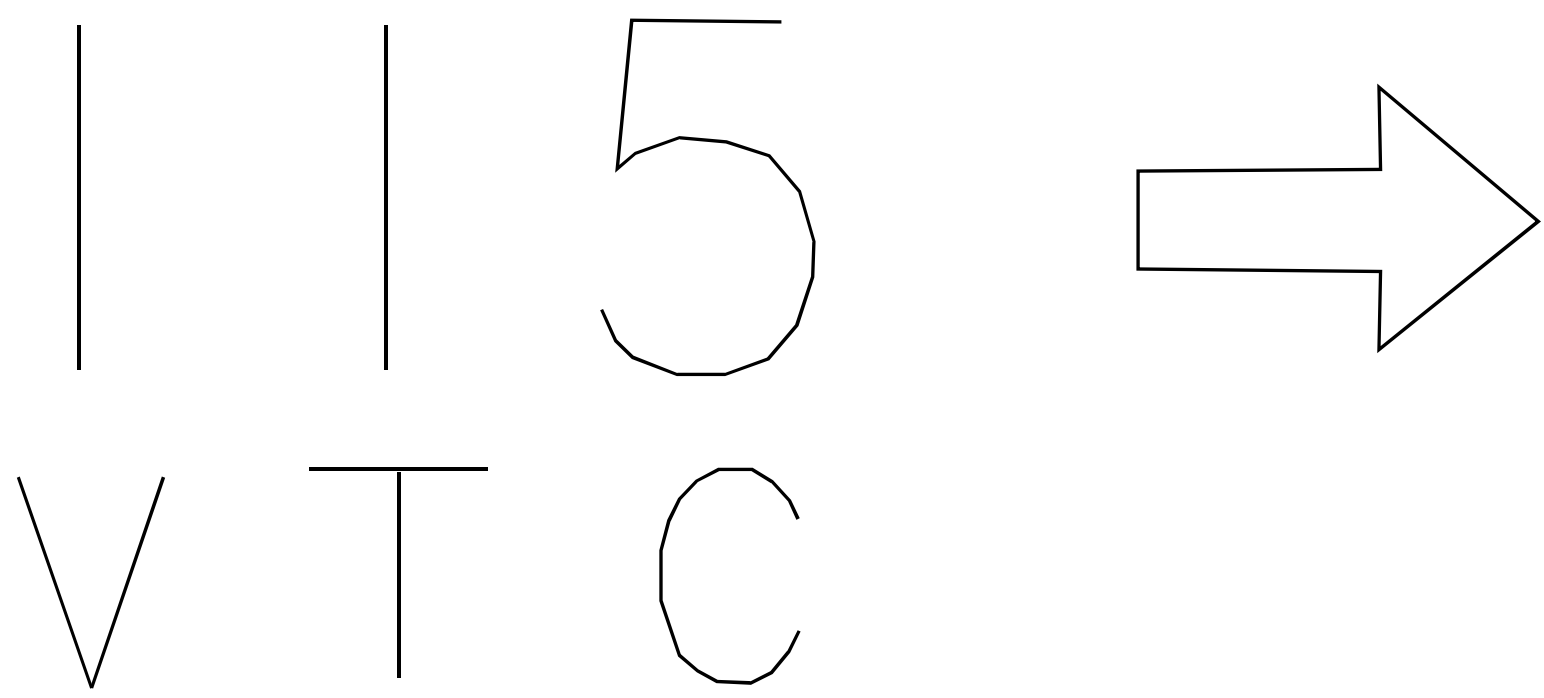

(a)

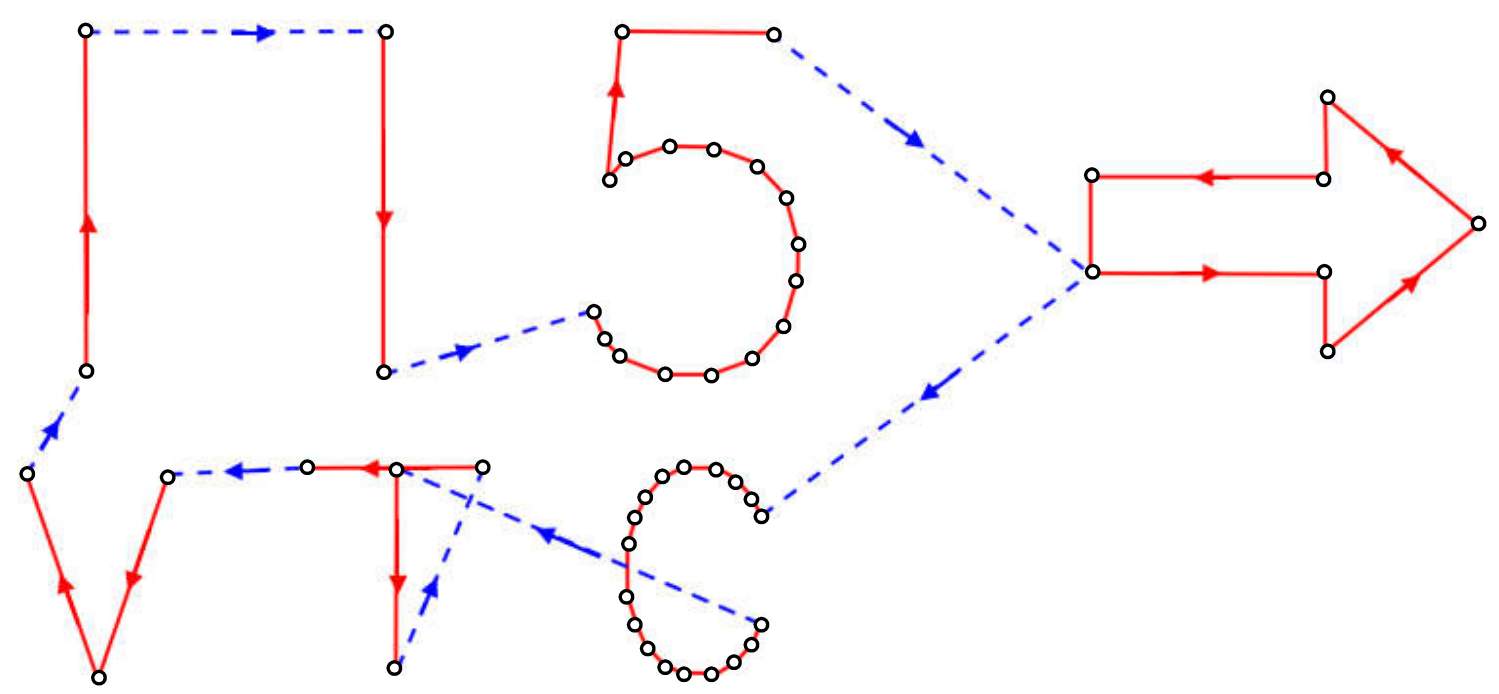

(b)

Fig. 5.9 (a) Desired vector pattern (b) Visible and transitional paths of the desired vector pattern.

A designed trajectory is shown in Fig. 5.9 (a). It is composed of visible and transitional paths. The scanning directions are also marked in the figure. Visible paths are the trajectories of the desired pattern and transitional paths are the trajectories (usually lines) that are used to connect the discrete desired patterns, which are shown as solid red and blue segments in Fig. 5.9. Both visible and transitional paths are composed of lines and curves. The curves are imitated by multiple very short line segments such as letter "C" in Fig. 5.9. Lines refers to relative long line segment such as digit " 1 " and lectter "V". The laser is turned on for the visible paths and turned off for the 
transitional paths, as a result, only the desired patterns will be observed in the display. Among all the segments, one segment is defined as the start segment whand its previous segment is defined as the end segment. All the segments are directional and are sequenced from the start segment to the end segment. The directions of the segments determine the scanning direction of the laser beam. There are two endpoints in one segment, by following the direction of the segment, the endpoint that starts the segment is defined as the start point and the the one that ends the segment is defined as the end point. The endpoints of all the segments are the critical points which define the main shape of the pattern and are shown as circles in Fig. 5.9 (b).

The micromirror steers the laser beam to scan through the trajectory by following a series of points such as the critical points. However, the critical points due to their small amount of numbers and coarse distributions have very poor control capbility of the the micromirror to compensate the undesired dynamics when tracing the desired trajectories. With only the critical points being the control points, the display will suffer from large distortions caused by the dynamic response of the micromiror, i.e., large overshoot and slow response speed. As a result, it is necessary to insert more control points besides the critical points to compensate the undesired dynamics of the micromirror. The critical points and the inserted points together consist of the control points of the micromirror. The number of points that inserted for each line segment and their distribution need to be carefully decided so that the dynamic response of the micromirror will not casue significant distortion of the display. The approach to determine the number of points inserted for each segment and their distributions is discussed in the following section.

\subsection{Point allocation}

\subsubsection{Point number assignment}

After the trajectory is designed and the critical points are extracted, the total number of the segments is known. The total number of control points $N$ is provided as an empirical value. Then different point number allocation strategies are used to assign a certain number of points to the visible line segments, visible curve segment and transitional segment.

As discussed in section 5.1, the overshoot and the slow response speed of the micromirror dynamic performance are the main cause for the display distortion. It was discovered in chapter 4 that the overshoot of the presented micromirror can be almost eliminated by applying a relative low base voltage, i.e., $50 \mathrm{~V}$. However, it comes with the cost of a sluggish response speed of the 
micromirror due to the effect of squeezed film damping. Different segments due to their lengths and point distribution strategies require different amount of time for the laser beam to scan through. Usually when driving the micromirror for display, once the refreshing rate is determined, the time interval between sending out the corresponding driving voltages of the points is a constant. As a result, the number of points assigned to each segment also represents the amount of time assigned to each segment. The aim of point number assignment is to assign proper number of points to each segment so that there will be enough time for the micromirror to steer the laser beam to scan through the entire segment. The main factor that affects the required scanning time for each segment is the length of the segment. Usually the longer segments require more time than that of the shorter segments since larger displacements of the micromirror are required to scan the longer segments. However, if the length of the segment is too short, more time (or control points) is required since the average scanning speed is slower on the short segment than that on the longer segment. This is due to the fact that the electrostatic actuation torque provided by the actuator is proportional to the square of the driving voltage. The short segment has smaller voltage variation from its start point to the end point than that of the long segment, which hence result in smaller average actuation torque and then smaller average scanning speed.

As mentioned early the segments can be divided into visible segment and transitional segment. For the visible segment, in terms of whether the segment belongs to a straight line or a curve it can be categorized as line segment and curve segment. The three different types of segments (transitional segment, visible line segment and visible curve segment) are treated differently when assigning point number and distributing the points which will be discussed in the next section. For convenience, the visible line segment and visible curve segment are called line segment and curve segment hereafter.

The curve segments are used to imitate the curve which have very short lengths. A large number of curve segments are usually clustered together such as digit " 5 " and letter " $\mathrm{C}$ " shown in Fig. 5.9. When extracting the critical points which define the shape of the pattern a lot of control points have already been assigned to the curve and no extra points are needed. The number of point that assigned to the curves is denoted as $N_{c}$. The point number assigned to transitional segments and visible line segments needs to subtract $N_{c}$ from the total number of points $(N)$, i.e., $N-N_{c}$. 
In order to assign the number of points to transitional segments and visible line segments, the weight factor is introduced and a weight factor is assigned to each segment. The number of points assign to each segment is proportional to its weight factor. The weight factor assigned to each segment depends on both the length level and the length of the segment. Three length levels which are short, middle and long are defined to classify the segments. The ratio between the length of a segment, i.e., $l$, and the length of the longest visible line segment, i.e., $l_{\text {VisMax }}$ is used to measure the length level. For visible line segments the largest ratio of $l / l_{V i s M a x}$ is 1 and for transitional segments the largest ratio may be larger or smaller than 1 since the longest transitional segment may be shorter or longer than the longest visible line segment. Some thresholds are defined to classify the segments into different length levels. The length level classification of visible line segments is illustrated in Fig. 5.10.

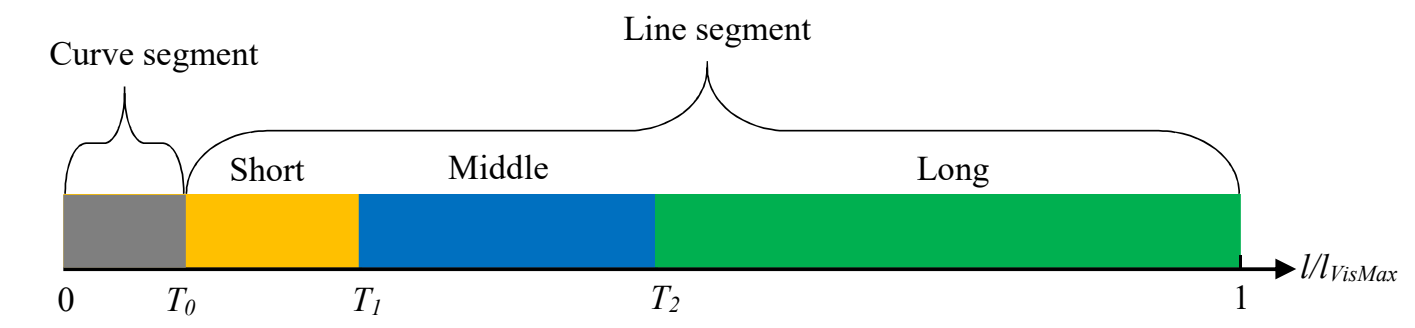

(a)

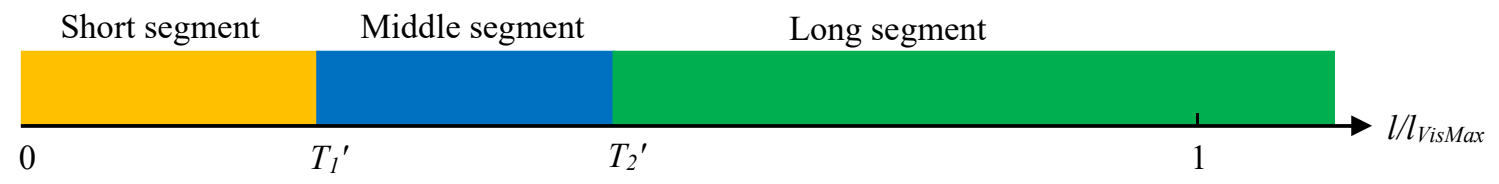

(b)

Fig. 5.10 (a) Length level classification of the line segments (b) Length level classification of the transitional segments.

Three thresholds $T_{0}, T_{1}$ and $T_{2}$ are used to define different levels of the segment length. The threshold $T_{0}$ is used to distinguish the curve segment from the line segment since they are much shorter than the visible line segments. The ratio $l / l_{V i s M a x}$ of the curve segment lies between 0 and $T_{0}$. Since the length of the curve segment is small, $T_{0}$ is usually equal or smaller than 0.15 . It is usually a constant for a pattern once the critical points are defined for the pattern, as shown in Fig. 5.9. If the ratio is larger than $T_{0}$ and smaller than $T_{1}$, the segment is defined as short and if the ratio is larger than $T_{2}$ the segment is defined as long. The segment whose ratio of $l / l_{V i s M a x}$ lies between 
$T_{1}$ and $T_{2}$ are defined as middle length. The similar classification can be made for transitional segments as shown in Fig. 5.10 (b) with thresholds $T_{1}{ }^{\prime}$ and $T_{2}$ '.

The weight factor assigned to each segment is proportional to the ratio of $l / l_{V i s M a x}$ and depends on its visibility (transitional segment or visible line segment) and length level. Two piecewise linear functions expressed in Eqs. (5.1) and (5.2) are designed to assign the number of points to the visible line segments and transitional segments respectively. There are 3 subdomains of each piecewise function which corresponds to 3 length levels, i.e., short, middle and long, as illustrated in Fig. 5.10. In Eqs. (5.1) and (5.2), $W_{V i s}(i)$ is the weight factor of the $i$ th segment if it is a line segment. $W_{\text {Tran }}(i)$ is the weight factors of the $i$ th segment if it is a transitional segment. $l(i)$ is the length of the $i$ th segment. $l_{V i s M a x}$ is the length of the longest visible segment. $T_{1}, T_{2}, T_{1}$ and $T_{2}$ are the thresholds which determines the length levels. $K_{s}, K_{m}, K_{l}$ and $K_{s}{ }^{\prime}, K_{m}{ }^{\prime}, K_{l}{ }^{\prime}$ are the parameters of two weight factor functions.

$$
\begin{gathered}
W_{V i s}(i)=\left\{\begin{array}{l}
K_{\mathrm{s}} \frac{l(i)}{l_{\text {VisMax }}}, T_{0}<\frac{l(i)}{K_{\text {VisMax }}} \leq T_{1} \\
K_{m} \frac{l(i)}{l_{\text {VisMax }}}, T_{1}<\frac{l(i)}{l_{\text {VisMax }}}<T_{2} \\
K_{l} \frac{l(i)}{l_{\text {VisMax }}}, T_{2} \leq \frac{l(i)}{l_{\text {VisMax }}}<1
\end{array}\right. \\
W_{\text {Tran }}(i)=\left\{\begin{array}{l}
K_{\mathrm{s}}^{\prime} \frac{l(i)}{l_{\text {VisMax }}}, 0<\frac{l(i)}{l_{\text {VisMax }}} \leq T_{1}^{\prime} \\
K_{m}^{\prime} \frac{l(i)}{l_{\text {VisMax }}}, T_{1}^{\prime}<\frac{l(i)}{l_{\text {VisMax }}}<T_{2}^{\prime} \\
K_{l}^{\prime}, T_{2}^{\prime} \leq \frac{l(i)}{l_{\text {VisMax }}}
\end{array}\right.
\end{gathered}
$$

In Eq. (5.1), $K_{s}, K_{m}, K_{l}$ are the slopes of the linear functions of three subdomains. Usually for the middle length level, $K_{m}$ is equal to 1 which indicates that the weight factor of the visible line segment that lies in the middle length level is simply the ratio of $l / l_{V i s M a x}$. As mentioned early, the short segment gets more weight than it deserves according to its length, i.e., more than $l / l_{\text {VisMax }}$, and the long segment get less weight than it should have got in terms of its length, i.e., less than $l / l_{V i s M a x}$. The actual weight factors for the visible line segments of each subdomains are controlled by $K_{s}, K_{m}$ and $K_{l}$ and they follow the relation that $K_{s}>K_{m}>K_{l}$. Similar to the visible line segment, 
in Eq. (5.2), $K_{s}{ }^{\prime}$ and $K_{m}{ }^{\prime}$ are the slopes of two linear functions with $K_{s}{ }^{\prime}>K_{m}{ }^{\prime} . K_{l}{ }^{\prime}$ is usually a constant which indicates that if the length of a transitional segment is long, the weight assigned to it does not increase with its length and remains a constant. This will reduce the weight of long transitional segments and enable more control points assigned to the short and middle segment. The reason is that the points on the transitional segment can have a nonlinear distribution due to its invisibility. The nonlinear distribution of the control points allow the laser beam to scan through the segment and stabilize at the end point with less control points (time) compared to that of the line segments whose control points is desired to be linearly distributed along the segment. The long transitional segment due to large ratio of $l / l_{V i s M a x}$ will have more than enough points assigned to it. As a result, a constant weight function in the long segment length subdomain will help to prevent from assigning redundant points to it. One example of the weight factor function for visible line segment is shown in Fig. 5.11. Where $T_{0}, T_{1}, T_{2}$ are 0.15, 0.25 and 0.5 respectively. $K_{s}, K_{m}, K_{l}$ are $1.4,1$ and 0.6 accordingly.

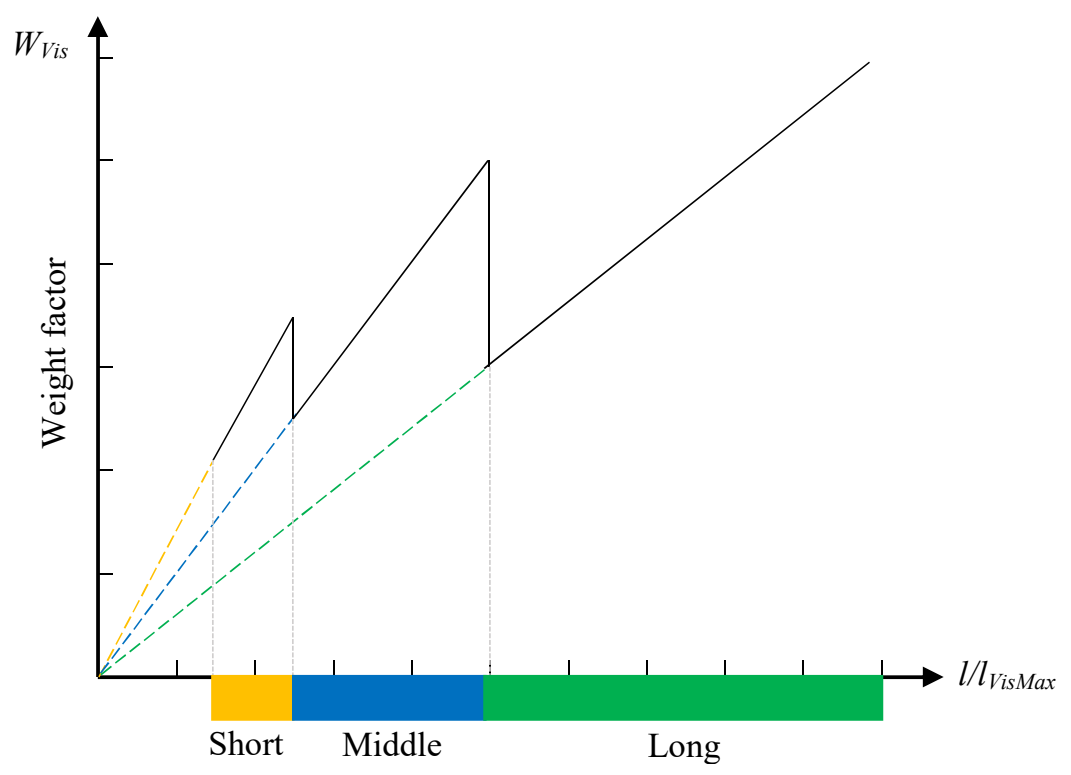

Fig. 5.11 One example of the distribution function expressed in Eq. (5.1)

After the weight factors are assigned to line segments and transitional segments, the proportion of the weight factor allocated to each segment in all the weight factors are calculated by Eq. (5.3).

$$
P(i)=\frac{W(i)}{\sum_{i=1}^{n} W(i)}
$$


Where $W(i)$ and $P(i)$ are the weight factor and the proportion of the weight factor of $i$ th segment. It is noted that due to the very small length, the curve segment has a fixed number of points assigned, i.e., 1 , as a result their weight factor are equal to zero. Then the exact number of points assigned to each segment is calculated in Eq. (5.4).

$$
n(i)=\left\{\begin{array}{l}
1, i \text { th segment is curve segment } \\
{\left[P(i) \cdot\left(N-N_{c}\right)\right], i \text { th segment is transitional or line segment }}
\end{array}\right.
$$

Where $n(i)$ is the point number allocated for the $i$ th segment. $N$ and $N_{c}$ are the total number of points and the point number allocated for curve segments. If the segment is curve segment, only one point is assigned to it and if the segment is transitional or line segment the point number allocated is calculated by multiplying the proportion of its weight factor $P(i)$ with the total number of points excluding the point number for the curve segments, i.e., $N-N_{c}$. Since the point number can only be integer, the result is rounded to the nearest integer.

\subsubsection{Design point distribution}

After each segment is assigned with the proper number of points, their distributions on the segments need to be determined. The the distributions of the control points need to be designed in a way that is able to compensate the slow response speed of the micromirror in scanning display.

\subsubsection{Point distribution}

The point distribution on the visible segments play a more important role than that on the transitional segments. Since the transitional segment is invisible and the only aim of the point distribution is to stablize the laser beam fast at its end point, a nonlinear distribution of the points can be designed. On the other hand, the distortion casued by the visible segments can easily be observed and the point distribution on them needs to be treated more carefully. From the display criteria discussed at the beginning of the chapter, the illuminance of the scanned visible segments should be uniform and at the same time the display should have a distortion as small as possible. In order to guarantee the uniformity of the illuminance of the display, the generic display approach previously prsented [123] adopts an even distribution of the control points along the visible segments. The even distribution works fine when the refreshing rate is relative low, i.e., about 30 Hz. However, a significant display distortion is present with the increase of the refreshing rate (refreshing rate) as shown in Fig. 5.12 below. 


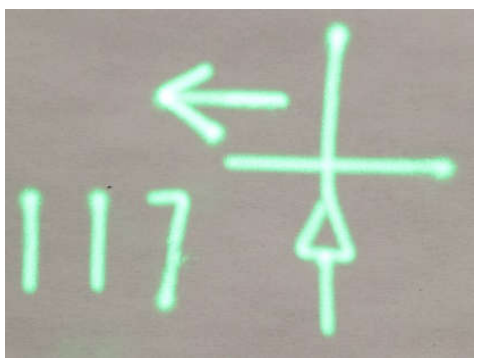

(a)

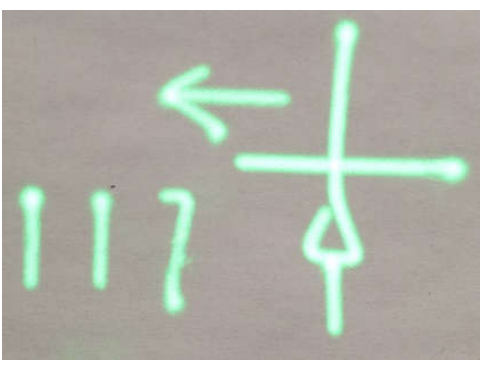

(b)

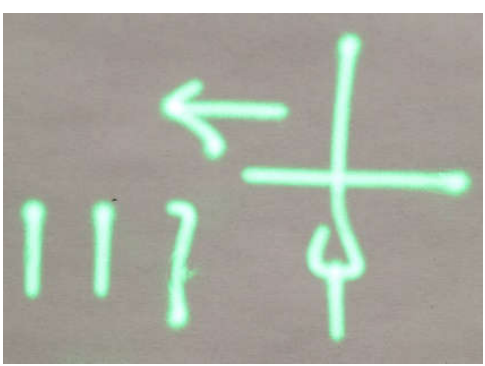

(c)

Fig. 5.12 Display distortion with the increase of the refreshing rate (a) $30 \mathrm{~Hz}$ (b) $40 \mathrm{~Hz}$ (c) $50 \mathrm{~Hz}$.

The main reason is that the even distribution in generic display approach has no flexibility of speeding up the response of the micromirror. With a higher refreshing rate, the laser beam has to scan through each segment with less time. A higher refreshing rate will lead to a significant lag between the driving signal and the actual location of the laser beam. Consequently, when the control signal for the next segment comes, the laser beam still has not reach or even far away from the start point of the next segment, which will lead to the distortion of the display.

In order to prevent the overshoot from causing the display distortion, a relative low base voltage, i.e., $50 \mathrm{~V}$, is used to drive the TRF micromirror. As a result, in operation, the micromirror plate is close to its underlying layer and exbits an overdamped behavior which is due to the significant squeezed film air damping. From the dynamic study of the micromirror in chapter 4 under $50 \mathrm{~V}$ base voltage, the point to point rotation of the micromirror has negligible overshoot but a long rise time, i.e., slow response speed. Since the generic approach lacks the flexibility to improve the scanning time for each segment, an advanced point distribution methodology is developed in this section for both the visbile segmnets and the transitional segments so that the scanning time for each segment can be adjusted and a good quality display can be obtained even for higher refreshing rate, i.e., $\geq 40 \mathrm{~Hz}$.

The point distribution method for the visible segments is illustrated in Fig. 5.13. Assuming that there are $n$ points assigned to a line segment, e.g., $\mathrm{n}=6$ in Fig. 5.13. Among the assigned $n$ points, 2 points are the start and end points of the segment, e.g., point 1 and point 6 in Fig. 5.13. The rest of the points are the inserted points which are added in addition to the start and end points. The last second point is defined as the peak point, e.g., point 5 in Fig. 5.13, which lies on the extension line of the segment following its direction and has the largest distance with the start point among all the points distributed on the segment. The rest of the inserted points are then evenly distributed 
between the start point and the peak point. Thanks to the peak point, the step size between the inserted points are increased compared to those generated using the generic display approach and have an even distribution. The point distribution generated using the generic approach is also shown in Fig. 5.13 as grey dashed lines and dots for comparison. The last point is assigned to the end point of the segment which guarantees the location of the laser beam lies at the end point at the end of the scanning. The larger slope of the black solid line compared to the grey dashed line in Fig. 5.13 indicates that the points distributed using the proposed approach have a larger step size. It determines that the voltage variations between the points are also larger than those of the points which are evenly distributed. Also since the electrostatic torque provided by the actuators is proportional to the square of the driving voltage, a larger voltage variation between the points will lead to larger increment of the actuation torques which accelerate the micromirror faster. The distance that the peak point goes beyond the end point is defined as the overshoot. Usually the percentage of overshoot is used to describe the magnitude of the overshoot, which is the ratio between the overshoot of the peak point and the length of the segment. The proposed distribution approach can be widely used for the visible line segments. It is guaranteed in the point number assignment that all the visible line segments have more than two points assigned so that the above mentioned distribution approach can be applied. The distribution function is expressed in Eq. (5.5).

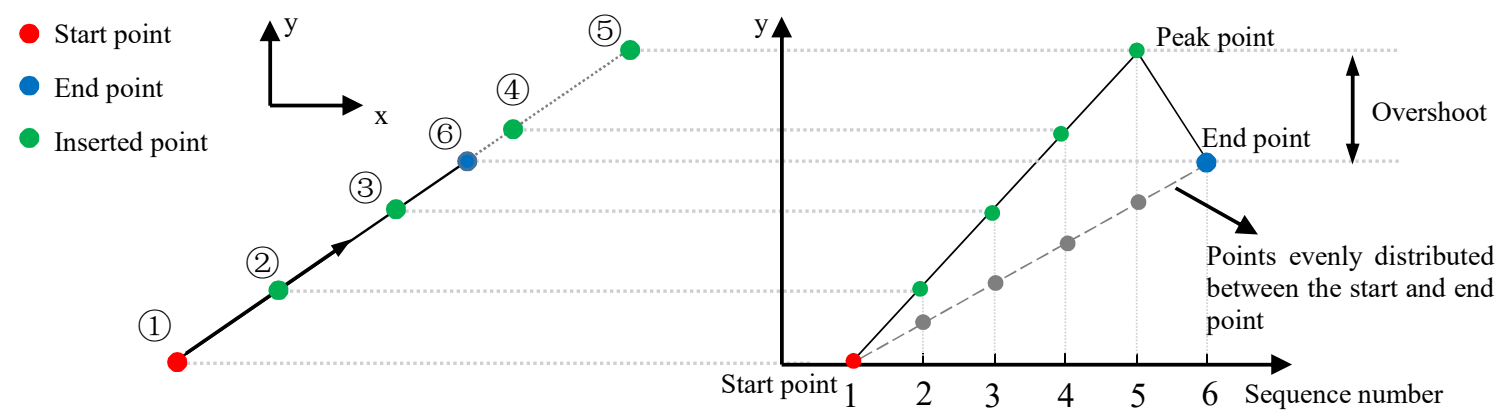

Fig. 5.13 Point distribution on visible line segment.

$$
f_{\text {VisLine }}^{i}(k)=\left\{\begin{array}{l}
p t_{i 0}, k=1 \\
p t_{i 0}+(k-1) \cdot\left(1+O s_{\text {VisLine }}(i)\right) \cdot \frac{p t_{i 1}-p t_{i 0}}{n(i)-2}, 2 \leq k \leq n(i)-1 \\
p t_{i 1}, k=n(i)
\end{array}\right.
$$


Where $f_{\text {VisLine }}{ }^{i}(k)$ is the distribution function for the $i$ th segment if it is a visible line segment. $k$ is the sequence number of the point that is distributed on the $i$ th segment which is from 1 to $n(i)$. $n(i)$ is the total number of points assigned to the segment. $p t_{i 0}$ and $p t_{i 1}$ are 2-element vectors which are the coordinates of the start and end points of the $i$ th segment. Os visLine $(i)$ is the percentage of overshoot of the peak point on the visible line segment. It is an adaptive factor that changes with the segment length and the angle between the $i$ th segment with its previous segment. The design of the adaptive function Os VisLine will be discussed in the next section.

The visible curve segments due to their small lengths have only one point assigned for each segment. They are treated as a special case of the visible line segment with the only point being the peak point which is located on the extension line of the segment. The distribution function is expressed in Eq. (5.6).

$$
f_{\text {VisCurve }}^{i}=p t_{i 0}+\left(p t_{i 1}-p t_{i 0}\right) \cdot\left(1+O s_{\text {VisCurve }}\right)
$$

Where $f_{\text {VisCurve }}{ }^{i}$ is the distribution function for the $i$ th segment if it is a curve segment. $p t_{i 0}$ and $p t_{i 1}$ are 2-element vectors which are the coordinates of the start and end points of the curve segment. Os VisCurve $_{\text {is }}$ the percentage of the overshoot of the peak point which is usually a constant for all the curve segments since their lengths are similar. It is noted that instead of assigning only one point, two points are assigned to the first curve segment of a cluster of curve segments to avoid from losing the start point of the first curve segment. Between the two points, the first one is the start point of the curve segment and the allocation of the second point follows Eq. (5.6).

As mentioned above, the response speed of the scanning laser beam can be improved by increasing the step size between the neighboring control points. Since the transitional segments are invisible, a more aggresive strategy can be used to distribute the control points on them. Instead of distributing points between the start and peak point on the visible line segment, the points of the transitional segment are distributed between the peak point and the end point as illustrated in Fig. 5.14. The first and the last point of the control points are assigned to the start and end point of the segment. The start point is followed by the peak point which goes beyond the end point. All of the inserted points overshoot beyond the end point and are distributed between the peak point and the end point which approach to the end point exponentially. With the points following the peak point approaching the end point exponentially, the laser beam will be finally stabalized at the end point. Instead of increasing the control voltages gradually in a step by step manner as on the visible line 
segment, a large voltage variation happens at the beginning of the scanning of the transitional segment and the large voltage is maintained during the whole scanning process. Consequently, the laser beam will have a larger accelertation on the transitional segment than the visible segment of the same length. The distribution function is expressed in Eq. (5.7).

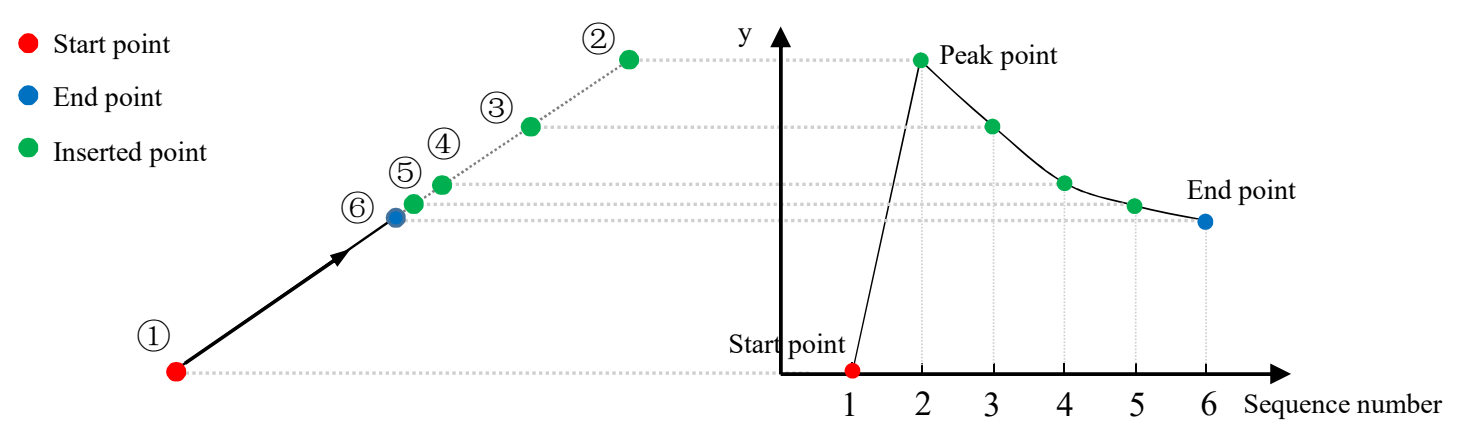

Fig. 5.14 Point distribution on transitional segment.

$$
f_{\text {Tran }}^{i}(k)=p t_{i 0}+\left(p t_{i 0}-p t_{i 1}\right) \cdot\left(1+O s_{\text {Tran }}(i)-\left(1-\exp \left(-6 \cdot \frac{k-1}{n(i)-1}\right)\right) \cdot O s_{\text {Tran }}(i)\right)
$$

Where $f_{\operatorname{Tran}}{ }^{i}(k)$ is the distribution functions for $i$ th segment if it is a transitional segment. $k$ is the sequence number of the point that is distributed on the $i$ th segment which is from 1 to $n(i) . n(i)$ is the total number of points assigned to the segment. $p t_{i 0}$ and $p t_{i l}$ are 2-element vectors which are the coordinates of the start and end points of the $i$ th segment. Os $\operatorname{Tran}(i)$ is the percentage of overshoot of the peak point which is also an adaptive parameter which changes with the length of the transitional segment.

\subsubsection{Overshoot function design}

In the distribution functions of the visible line segment and the transitional segment, the percentage of overshoot of $O_{S_{V i s L i n e}}$ and $O_{s_{\text {Tran }}}$ change with different segments. The percentage of overshoot indicates the control effort that is required for each segment. If a segment requires more control effort of the micromirror, it implies that a larger percentage of overshoot needs to be assigned for the segment. The control effort required for each segment is related to the length of the segment and its angle with the previous segment. The aim of this section is to design the functions of Os VisLine $_{\text {and }} O_{\text {Tran }}$ so that each segment can be assigned with a proper amount of the percentage of overshoot which further leads to less distorted display in high refreshing rate. 
The control effort for each segment is determined by its angle with the previous segment and its segment length. The effect of the angle with the previous segment is illustrated in Fig. 5.15. In Fig. 5.15, two intersected line segments with equal length and different intersection angles of $30^{\circ}$, $90^{\circ}$ and $120^{\circ}$ are shown in (a) - (c) which are assumed to be the desired scanning trajectory of the laser beam. The scanning direction is from point $\mathrm{A}$ to $\mathrm{C}$ through $\mathrm{B}$ which is the intersection point. In order for the laser beam to follow the segment, its velocity should be in the same direction as the segment. Usually when the laser beam reaches the intersection point, e.g., B, there is a residual speed which has the same direction as the scanning direction of the previous segment. Assume that $\mathrm{B}^{\prime}$ is a point on $\mathrm{BC}$ and has the same magnitude of the velocity as $V_{B}$. In order for the scanning velocity to change from $V_{B}$ to $V_{B^{\prime}}$, a larger velocity variation is required for the case which has a larger intersection angle of the segments as illustrated in Fig. 5.15 (d). Also since the velocity variation $(\Delta V)$ and the acceleration ( $a$ ) has the relation $a=\Delta V / t$, for the same amount time $t$, the required acceleration and then actuation force/torque increases with the increase of the intersection angle. The actuation torque is a measure of the control effort and the larger the required actuation torque is, the larger control effort that is needed for the scanning.

More specifically, if the intersection angle is smaller than $90^{\circ}$ as shown in Fig. 5.10 (a), a small positive residual speed makes the scanning of the second segment faster since residual speed has a velocity component along the second segment, which has the same direction as the scanning direction of the second segment. It provide an initial velocity for the scanning of the second segment and makes it faster. However, if the turning angle is larger than $90^{\circ}$ as shown in Fig. 5.15 (c), the residual velocity will have an opposite direction with the desired scanning direction of the second segment. In order to achieve a same scanning time for the second segment as the former case, larger actuation force is required. In this case, significant control effort is required to fast scan the intersected segments with larger than $90^{\circ}$ intersection angle. From the analysis above, the effect of the intersection angle to the percentage of overshoot assigned to the peak point of the line segment is expressed in Eq. (5.7). 


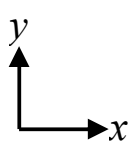

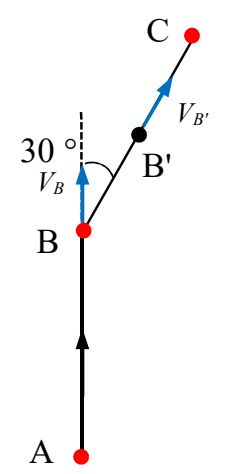

(a)

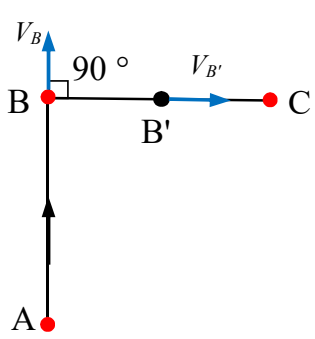

(b)

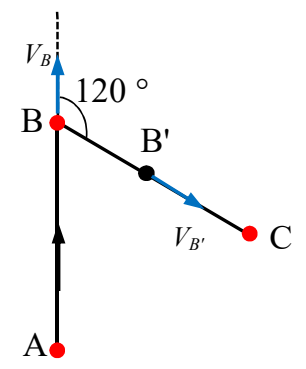

(c)

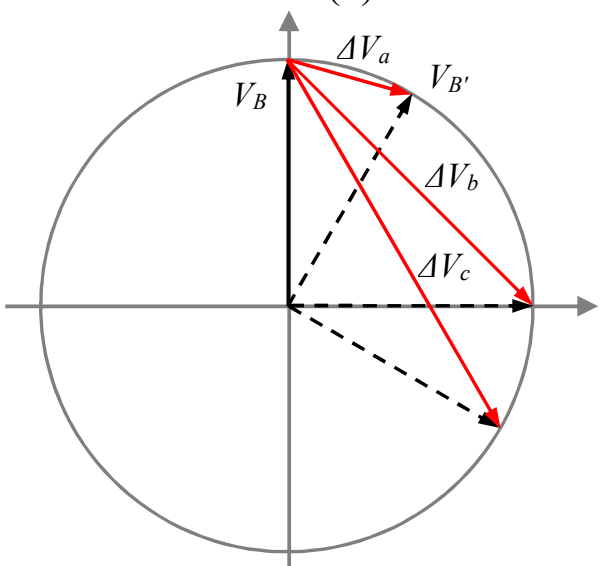

(d)

Fig. 5.15 (a) (c)Two intersected segments with intersection angles $30^{\circ}, 90^{\circ}$, and $120^{\circ}$ respectively (d) Velocity variation from $V_{B}$ to $V_{B}$ ' of the three cases illustrated in (a) - (c).

$$
O S_{\text {VisLine }}(i)=\left\{\begin{array}{l}
\frac{\alpha}{l(i)}+\frac{\theta(i)}{180}, l(i)<L_{T} \text { and } \theta(i)>90^{\circ} \\
\frac{\beta}{l(i)}, l(i) \geq L_{T} \text { or } \theta(i) \leq 90^{\circ}
\end{array}\right.
$$

Where OsvisLine $(i)$ represents the percentage of overshoot of the peak point of $i$ th segment if it is a visible line segment. $\alpha$ and $\beta$ are two parameters that control the percentage of the overshoot. $l(i)$ and $\theta(i)$ are the length of the $i$ th segment and its intersection angle with the previous segment. If the length of segment is not long enough, i.e., smaller than the threshold $L_{T}$ and the intersection angle is larger than $90^{\circ}$ the extra control effort brought by the intersection angle needs to be considered as a variable term besides a constant overshoot, i.e., $\alpha$, as shown in the first equation in Eq. (5.7). The rest of the segments are applied a constant overshoot, i.e., $\beta$. 
As mentioned early, the transitional segments have much larger tolerance on the point distribution than the visible line segment. As a result, the effect of the intersection angle on the percentage of overshoot can be neglected and is simply related to the length of the segment as expressed in Eq. (5.8).

$$
O s_{\text {Tran }}(i)=-O s_{\text {TranMax }} \cdot \frac{l(i)}{l_{\text {TranMax }}}+O s_{\text {TranMax }}
$$

Where $\operatorname{Os}_{\operatorname{Tran}}(i)$ is the percentage of overshoot of $i$ th segment if it is a transitional segment. $l_{\operatorname{TranMax}}$ is the maximum length of the transitional segment. Os TranMax is a constant which is the maximum percentage of overshoot of the transitional segment. Following Eq. (5.8) a longer transitional segment is allocated a smaller percentage of overshoot.

\subsection{Control voltage generation and parameter identification}

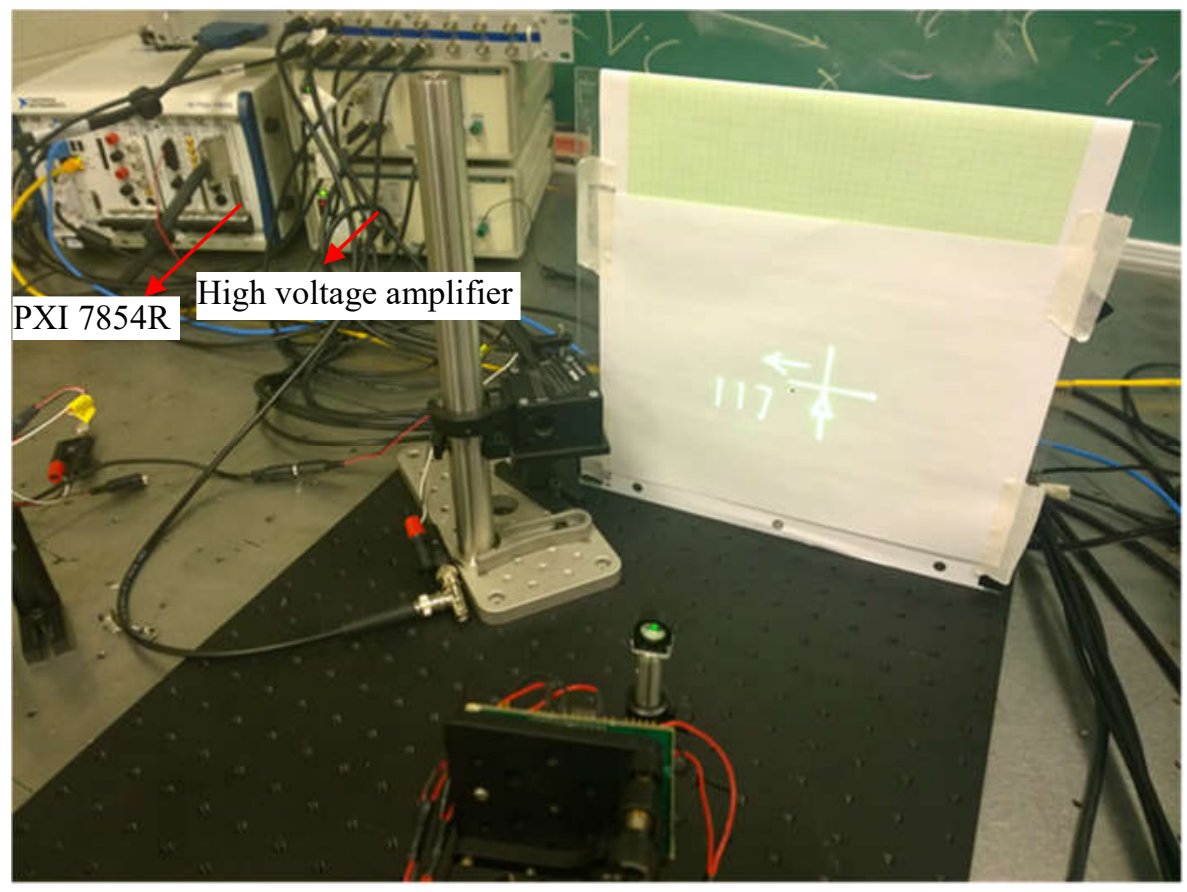

Fig. 5.16 Display setup.

The method of assigning corresponding driving voltages to each of the control point is similar to the one discussed in [123]. MATLAB surface fitting toolbox is used to create a calibration model for each of the actuator separately using the data from the calibration matrix. Then the control points are fed into the calibration model to obtain the driving voltages for the control points. 
The voltage signals are sent out by PXI 7854R FPGA data acquisition card and then are amplified by high voltage amplifier and then applied to the micromirorr as shown in Fig. 5.16.

The control voltages generated by the advanced approach is used to drive the micromirror to display various patterns and the design parameters in the point number assignment, i.e., $T_{1}, T_{2}, T_{1}{ }^{\prime}$, $T_{2}{ }^{\prime}, K_{s}, K_{m}, K_{l}, K_{s}{ }^{\prime}, K_{m}{ }^{\prime}, K_{l}{ }^{\prime}$ and point distribution, i.e, $\alpha, \beta, L_{T}, O_{\text {TranMax }}$ and Oscurve are found from trail and error methods. It has been found that all the parameters for the point number assignment and some of the parameters for point distribution are consistant for different display patterns and the optimal parameters found are listed in the Table 5.1. $\alpha$ and $\beta$ are two parameters that are mainly used to tune the display quality. They change with the display pattern and refreshing rate.

Table 5.1 The optimal parameters obtained using tral and error methods.

\begin{tabular}{|c|c|c|c|c|c|c|c|c|c|c|c|}
\hline \hline$T_{1}$ & $T_{2}$ & $T_{1}{ }^{\prime}$ & $T_{2}{ }^{\prime}$ & $K_{s}$ & $K_{m}$ & $K_{l}$ & $K_{s}{ }^{\prime}$ & $K_{m}{ }^{\prime}$ & $K_{l}^{\prime}$ & $O_{\text {Curve }}$ & $L_{T}$ \\
\hline 0.25 & 0.5 & 0.4 & 0.5 & 1.4 & 1 & 0.6 & 1.25 & 1 & 0.5 & 2.5 & $0.6 \cdot$ DisVis $_{\max }$ \\
\hline \hline
\end{tabular}

\subsection{Display results}

The voltages generated using advanced display approach and using the optimal parameters obtianed are used to drive the micromirror to display. The display pattern shown in Fig. 5.8 is displayed with different frequencies i.e., from $30 \mathrm{~Hz}$ to $50 \mathrm{~Hz}$. The display pattern can be used for automotive HUD which is composed of 3 digits " 117 " and a simple map of the road, i.e., the two orthogonal lines. The triangle indicates the current location of the vehicle. The control voltages generated using the generic display approach presented in [109], [123] are also used to control the same micromirror to display the same patterns under the same display frequencies. Comparisons are maded between the display results which are shown in Figs. $5.17 \sim 5.19$.

It can be observed from Fig. 5.17 that sharp corners can be well displayed such as those in digit "7" and the triangle shape. However, the corners are all rounded in the display using generic display approach. This indicates that the presented approach is able to compensate for the slow response speed of the micromirror under $50 \mathrm{~V}$ base voltage. With the refreshing rate increase, it can be concluded from Figs. $5.18 \sim 5.19$ that the display results using the generic display approach deteriorate significantly. However, the advanced display approach is able to adjust the design parameters $(\alpha$ and $\beta$ ) to obtain much better quality display. Namely, the advanced display approach has the flexibility to adjust the design parameters to obtain good quality displays under different 
refreshing rate (especillay in high frequencies), which cannot be achieved using generic display approach.

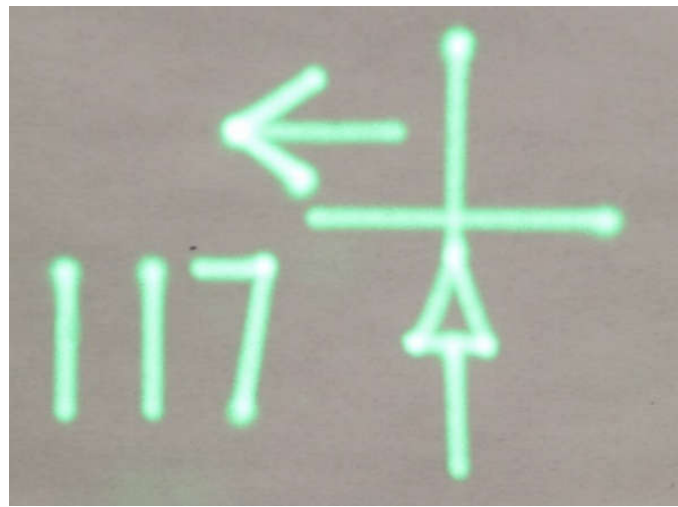

(a)

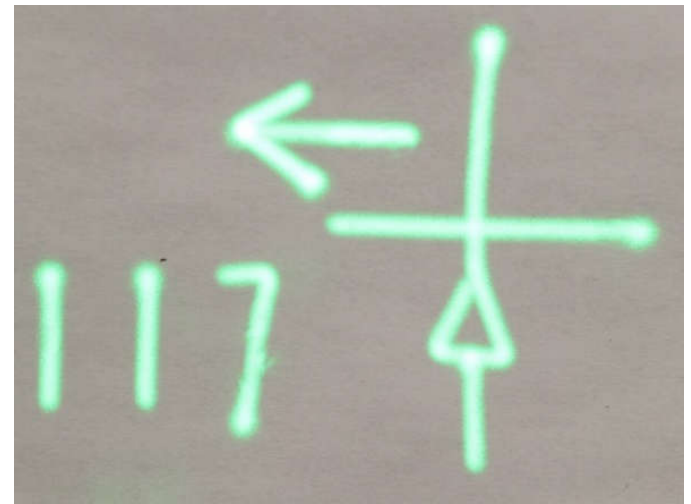

(b)

Fig. 5.17 Display in $30 \mathrm{~Hz}$ (a) Advanced display approach (b) Generic display approach.

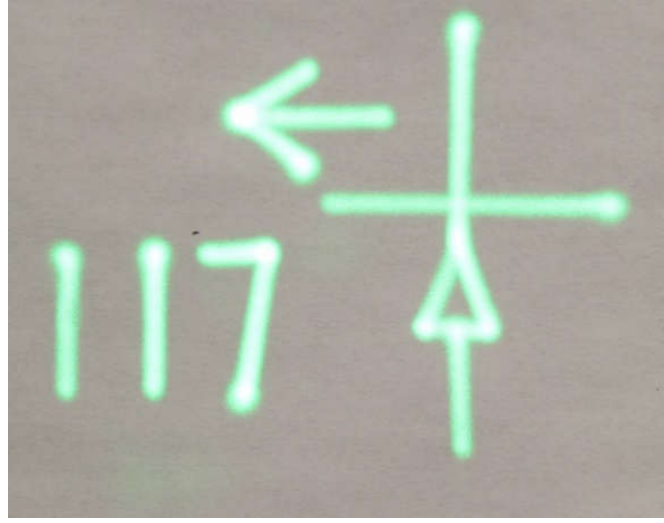

(a)

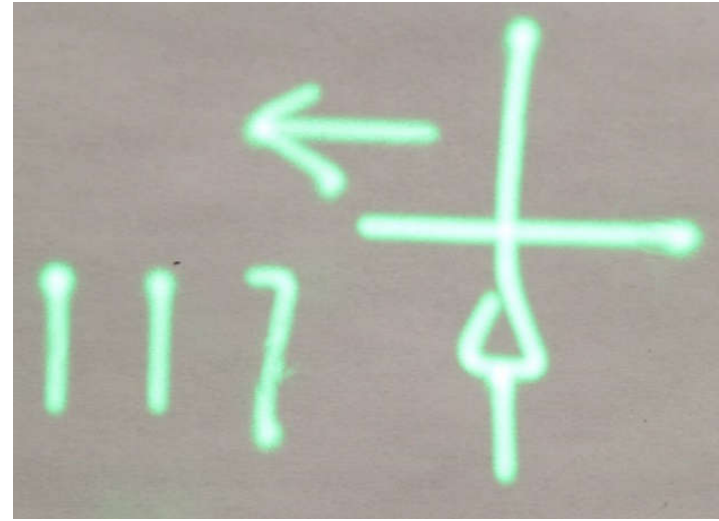

(b)

Fig. 5.18 Display in $40 \mathrm{~Hz}$ (a) Advanced display approach (b) Generic display approach.

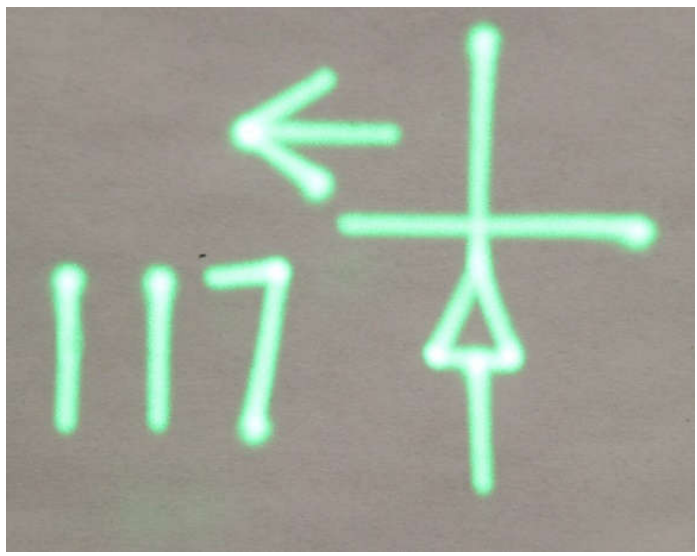

(a)

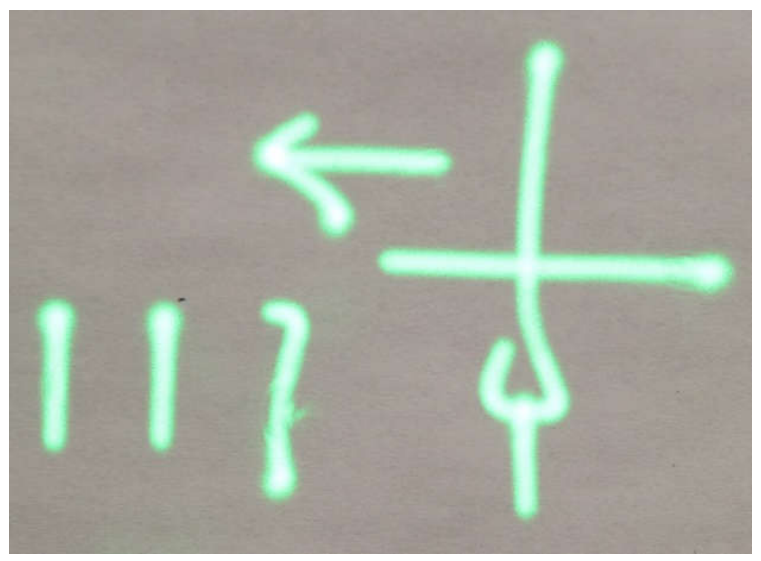

(b)

Fig. 5.19 Display in $50 \mathrm{~Hz}$ (a) Advanced display approach (b) Generic display approach. 
The advanced display approach not only can improve the display quality of the micromirror presented in chapter 4, but also it can be used on the previously developed ORF micromirror to improve the display quality. A display module based on the ORF micromirror was previously developed. Its display result using the generic display approach with refreshing rate about $43 \mathrm{~Hz}$ is shown as the left display pattern of Fig. 5.20. The designed display pattern is almost the same as the one shown in Fig. 5.9 except that the arrow is designed to be closer to the digits. It is noted that the generated control voltages are amplified by a scale factor to improve the response speed. The advanced display approach is used to display the pattern designed in Fig. 5.9 so that the edge of the arrow and the digit " 5 " can be clearly identified. The display result is shown as the right display of Fig. 5.20. In order to avoid the influence of the viewer's subjective factor and make fair comparison between the two display results, some comparison guidelines are made as follows. A good display result should have a shape closer to that of the designed pattern. More specificly, the straight lines of the designed pattern should be as straight as possible in a good display. The intersection angles between the segments should be preserved as much as possible, i.e., the sharp corners should stay as sharp as the original design. By using the above comparision guidelines it can be concluded that the advanced display approach can also improve the display quality of the previously developed micromirror. For example, for the display pattern in the right of Fig. 5.20 the straight lines of the letters "V" and "T" are straighter than those of the left display pattern. The arrow of the right display pattern preserves the sharp corners of the original design in Fig. 5.9 while the arrow of the left display pattern suffers from a siginificant distortion and all the sharp corners are rounded. It can also be observed that the advanced display approach has smaller improvement on the curves than on the straight lines. For example there is limited improvement on the letter "C".

Finally the display pattern shown in Fig. 5.9 is displayed using the TRF micromirror and the advanced display approach. The display result is compared with that of the previously developed display module based on the ORF micromirror and generic display approach. The comparison setup is shown in Fig. 5.21. The display module is positioned so that the micromirror inside is approximately aligned with the micromirror on the fixture. As a result, both micromirrors have the same distance to the screen and the size of the generated display can be directly compared. The comparison result in $43 \mathrm{~Hz}$ is shown in Fig. 5.22. It can be seen that the displayed pattern using 
TRF micromirror and the advanced display approach is much superior in both the size and the display quality than that of the ORF micromirror with generic display approach.

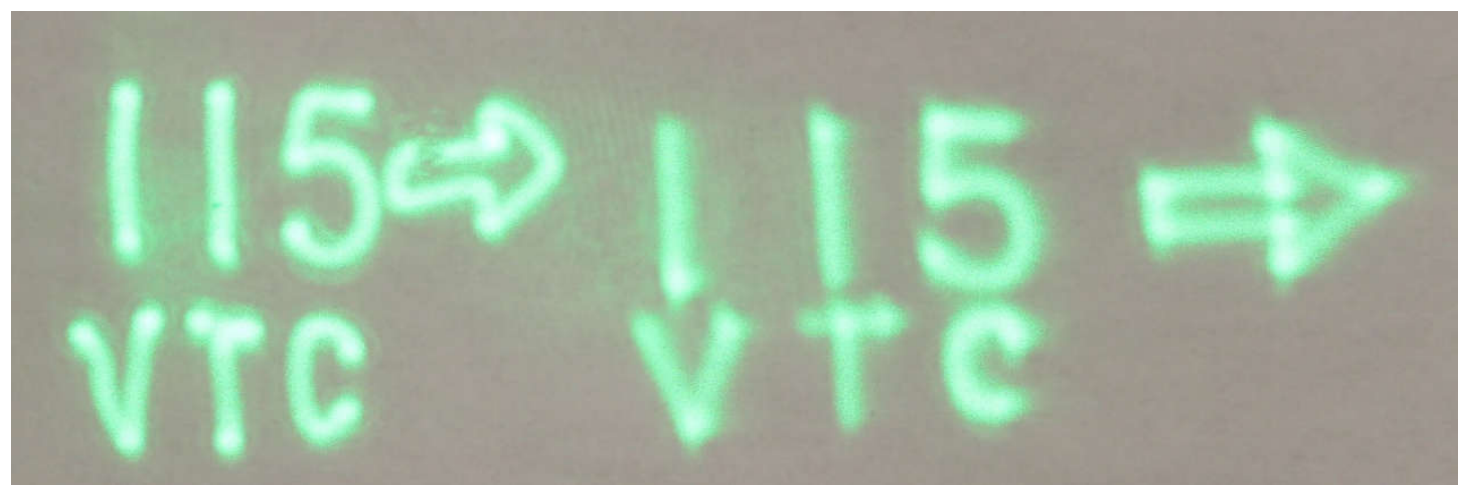

Fig. 5.20 Display results of the ORF micromirror in $43 \mathrm{~Hz}$. Left: Generic display approach.

Right: Advanced display approach.

\subsection{Summary}

From the experiments and discussions above, it can be concluded that the proposed advanced display approach can be widely used on the micromirrors of the similar type and is capable to achieve displays with much better display qualities than that using the generic display approach, especially in high display frequencies. The advanced display have large improvement on the line segments. However, the improvement of the display quality of the curve segments are limited. The TRF micromirror together with the advanced display approach can generate superior displays both in size and quality than that using the ORF micromirror and generic display approach. 


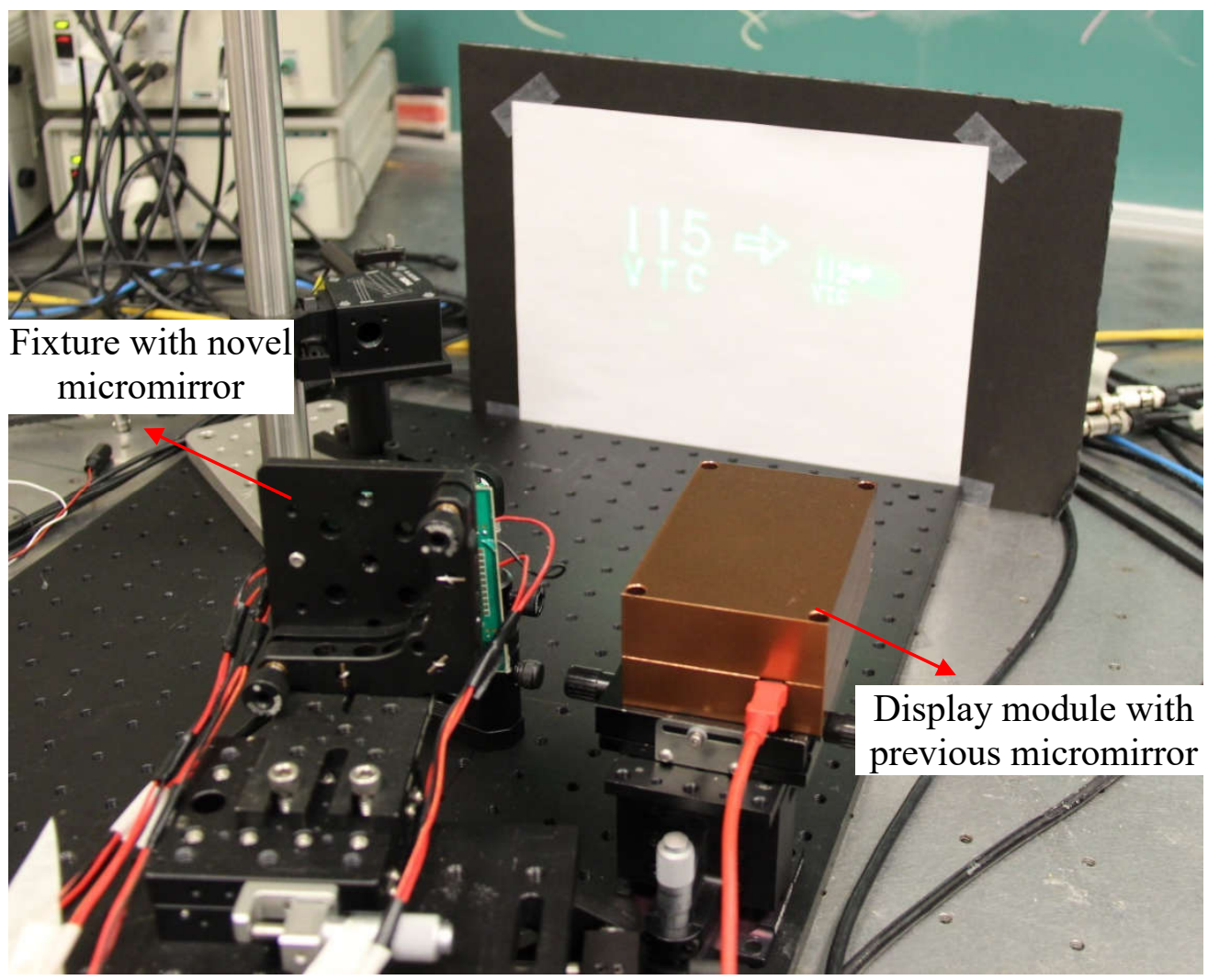

Fig. 5.21 Comparison setup.

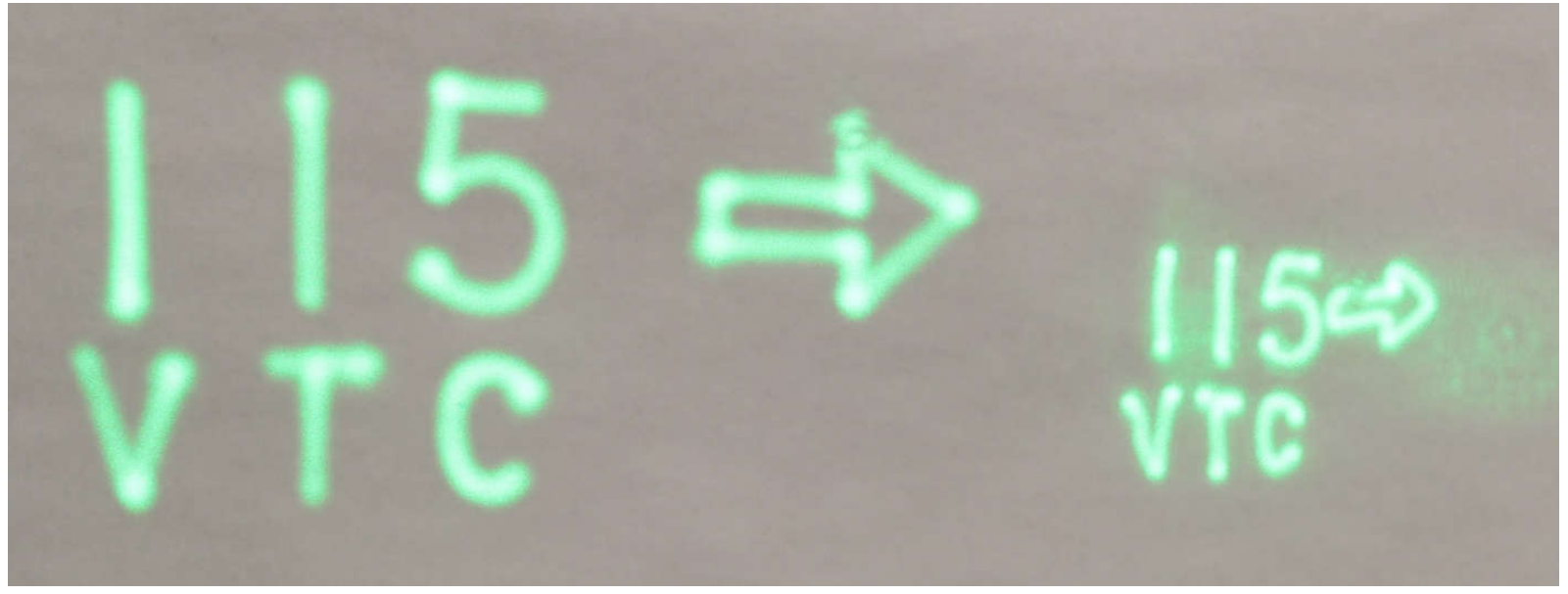

Fig. 5.22 Display result comparison using two micromirrors. 


\section{Chapter 6 Application of the micromirror on automotive head-up display (HUD)}

Part of this chapter is based on the following submitted paper:

Chao Fan and Siyuan He, "Micromirror Based virtual image automotive head-up display", Journal of microsystem technologies, 2015.

Part of this chapter is based on the following to be submitted paper:

Chao Fan and Siyuan He, "Micromirror based automotive head up display (HUD)", IEEE Transaction on Mechatronics.

One application of the micromirror based LSD that is getting more attentions is automotive HUD. This chapter meets the objective 3 of the thesis and constructs a HUD system on experimental cars using the micromirror presented in chapter 4 and advanced display approach presented in chapter 5. The display module is designed and fabricated, which integrates the micromirror and light source and optical components. Two configurations of automotive HUD, i.e., real image and virtual image HUD are built. The display results are shown. The light efficiency and vibration reliability are tested for the micromirror.

\subsection{Design and working principle}

\subsubsection{Display module}

The display module is composed of a laser diode module, a micromirror, and a double concave lens, as shown in Fig. 6.1. A collimated $532 \mathrm{~nm}$ laser diode module with modulation capability (up to $3 \mathrm{kHz}$ ) is used as the light source. The output power of the laser is about $3 \mathrm{~mW}$ with a beam diameter smaller than $1.2 \mathrm{~mm}$ which falls into class IIIa. A pinhole of $0.9 \mathrm{~mm}$ is bonded in front of the lens of the laser diode module which is used to reduce the diameter of the laser beam so that it fits the size of the micromirror plate (1 $\mathrm{mm}$ in diameter). The micromirror is packaged in a CQFP44 chip and is soldered on a small PCB board for wire connection. There is an anti-reflective (AR) coated glass bonded on top of the CQFP44 chip to seal the micromirror inside the chip cavity. This can protect the micromirror from being contaminated by the particles in the air. According to the optical simulation and optimization performed in chapter 4, a $12 \mathrm{~mm}$ diameter AR coated 
double concave lens with $12 \mathrm{~mm}$ effective focal length is used to magnify the scanning. The AR coating is able to provide smaller than $1.75 \%$ reflectance per surface for $400 \sim 700 \mathrm{~nm}$ [145].

A set of mechanical parts are designed to integrate all of the parts into a small device, which includes base plate, laser fixture, micromirror fixture and lens fixture. All fixtures are located on the base plate and provide 1D rotation with the rotational axis normal to the base plate. Besides the micromirror and lens fixtures are able to provide 2D translation in the base plane, vertical translation. All the fixtures work together to align the optical components with the laser beam. It is desired that the incident laser beam is aligned with the micromirror plate and the reflected laser beam is aligned in the center of the double concave lens. The schematic of the integrated device is shown in Fig. 6.1. By controlling the rotation of the micromirror, the reflected laser beam is able to scan through a designed trajectory repeatedly and rapidly. During the scanning, the laser is modulated to be turned on for the visible trajectories and turned off for the transitional trajectories. By combining the micromirror scanning and laser modulation, the desired laser patterns can be generated.

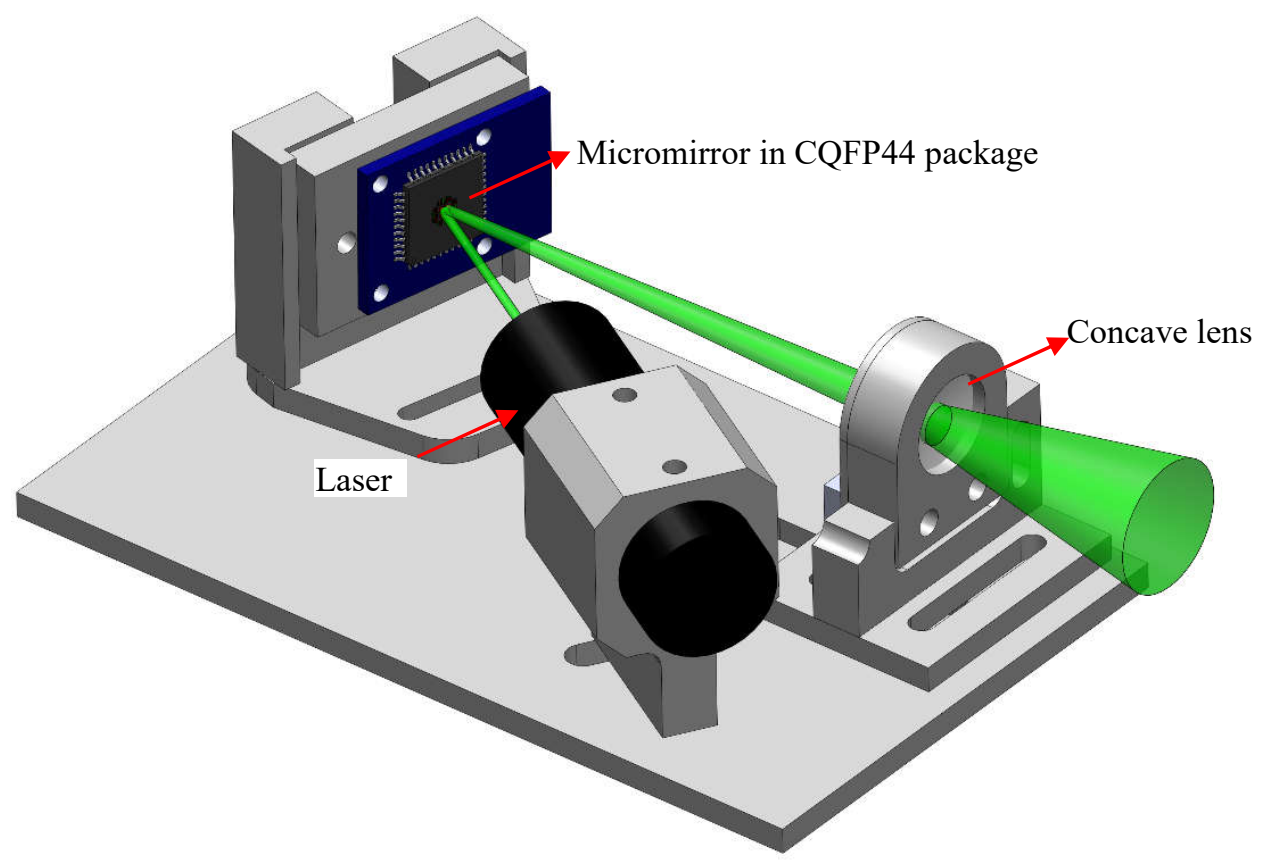

Fig. 6.1 Display module. 


\subsubsection{Real image and virtual image configuration of automotive HUD}

There are two configurations of the automotive HUD, i.e., real image (or direct projection) and virtual image HUD. Real image HUD generates a real image directly on the surface of a combiner or the windshield. On the other hand, virtual image HUD generates a virtual image in front of the divers' sightline which appears to be on the road in the front. The advantages of the real image HUD are simple configuration, high illuminance efficiency and is suitable for aftermarket installation. However it requires the driver to refocus to obtain the information, i.e., move focus from the road to inner surface of the windshield. On the contrary, the virtual image does not require the refocusing for the driver to obtain the HUD information. This comes with the sacrifice of the device volume and lower the illumination efficiency of the display module. Also due to the long optical path length required, it is not easy to be installed aftermarket.

The proposed real image HUD is shown in Fig. 6.2. The display module is directed to the windshield and can be positioned either on the dashboard or buried under it. A partial mirrorreflective transparent film is attached on the inner surface of the windshield with a diffusereflective transparent film stacked on top of it. The real image is generated on the surface of the diffuse-reflective film since the laser vector graphics projected on it illuminate to various directions. As a result, the pattern can be observed from a large angle. The underlying reflective film is used to reflect the transmitted light and enhance the brightness of the display.

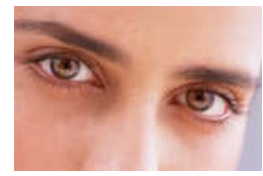

Driver

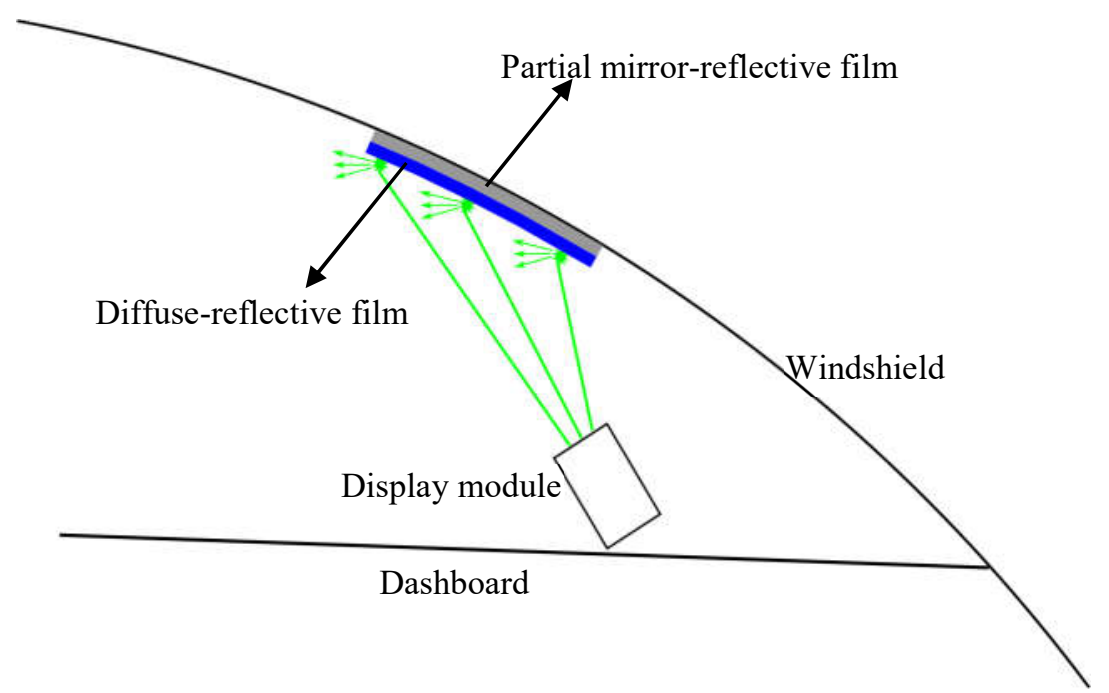

Fig. 6.2 The setup of the real image automotive HUD. 
The optical setup of the virtual image automotive HUD is shown in Fig. 6.3. The scanning laser beams from the display module is first reflected by a metallic coated reflective mirror. The angle of the reflective mirror is adjusted to reflect the laser beams onto a projection screen where a real image is formed. The real image is then partially reflected by a transparent HUD reflector which is placed on top of the dashboard. Consequently, a virtual image is generated in front of the HUD reflector. It is noted that the partially mirror-reflective film same as the one used as the bottom film in real image HUD configuration can also be coated on the inner surface of the windshield instead of using a HUD reflector.

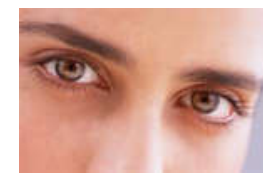

Driver

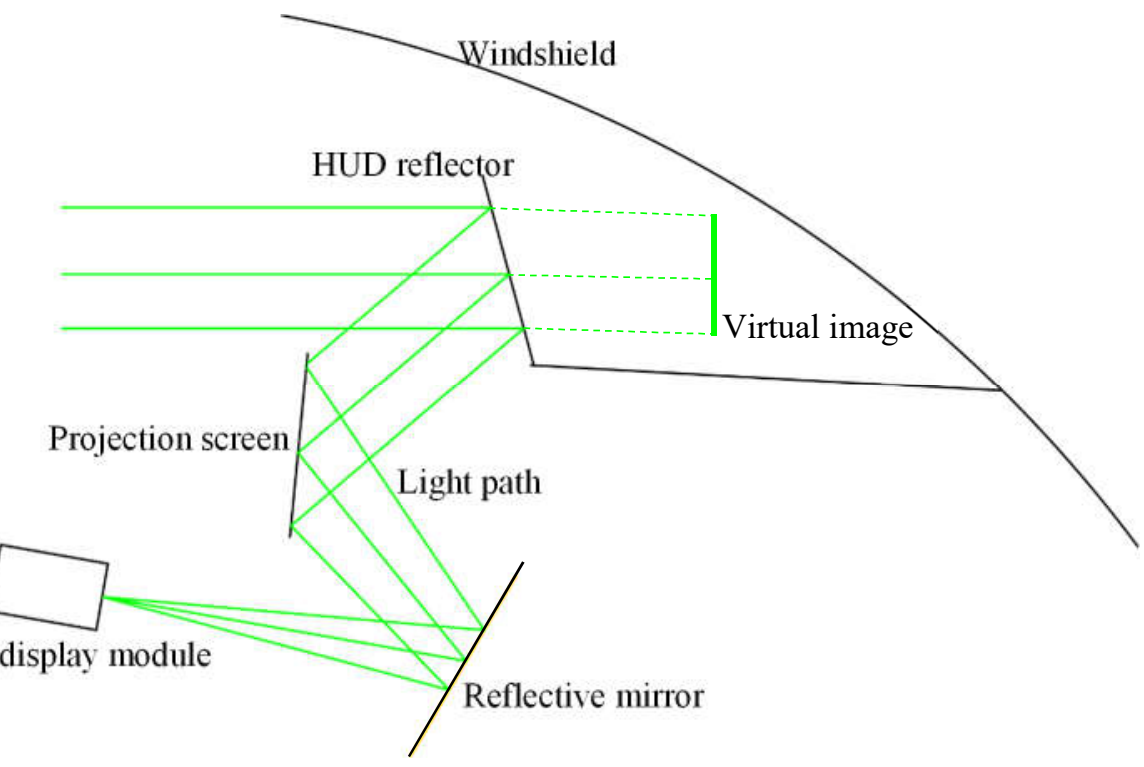

Fig. 6.3 Virtual image automotive HUD setup.

\subsection{Prototype}

\subsubsection{Display module}

The prototype of the display module is fabricated and is shown in Fig. 6.4 below. All the fixtures are fabricated using aluminum and can be fixed on the base by screws. The power supply of the laser, and the driving signals are provided using external equipments. The signal flow is shown in Fig. 6.5. The driving signals together with the laser modulation signal are generated using MATLAB software first using the advanced display approach presented in chapter 5 . Then the data is saved as MATLAB data file. It is noted that the control signals generated using MATLAB are low voltage $(0 \sim 5 \mathrm{~V})$. The PXI-7854R multifunction data acquisition board from National Instrument which can be programed using LabVIEW software and can interface with MATLAB 
data file is used to output the low voltage control signals. There are 5 channels of output signals which correspond to the four driving signals of the micromirror and a modulation signal for the laser. The generated signals are then amplified 50 times using high voltage amplifiers (model 2350 from Tegam). The amplified signals are then applied to the micromirror. The laser modulation signal is sent to the laser modulation control circuit which comes with the laser diode module.

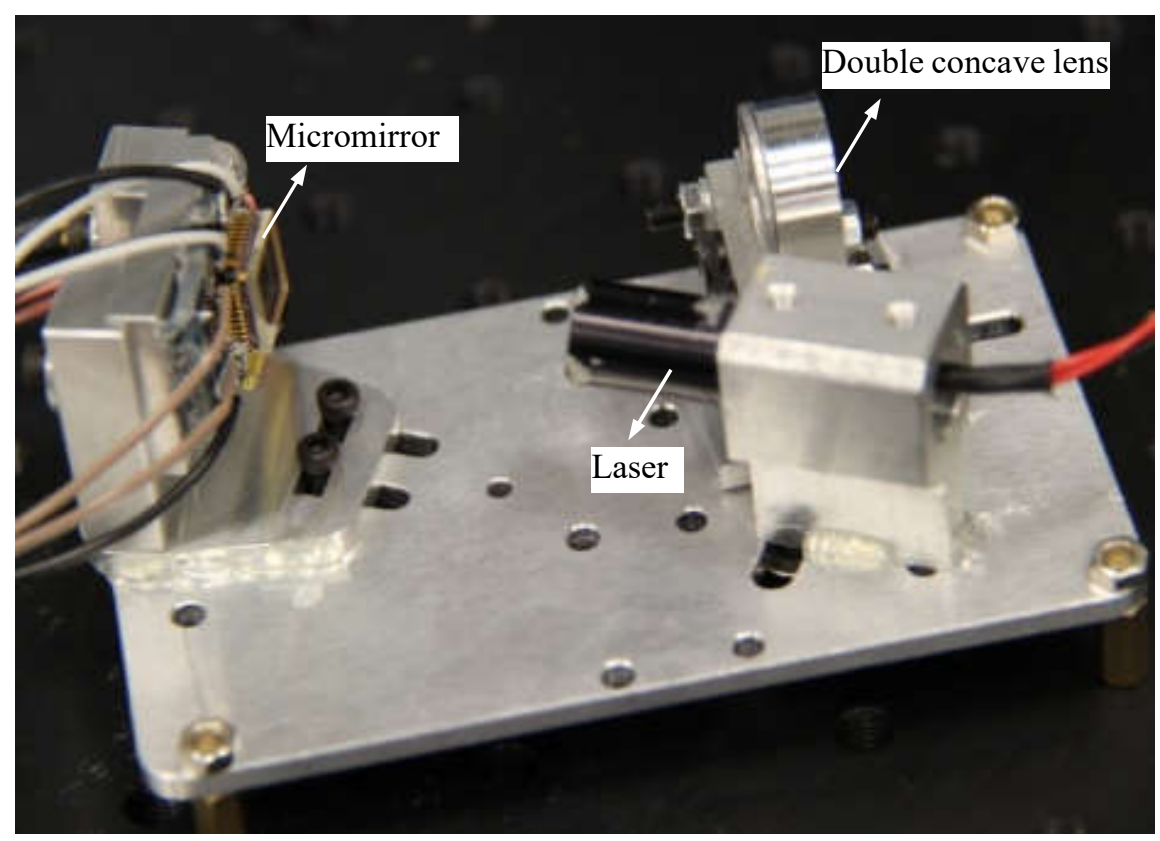

Fig. 6.4 The prototype of the display module.

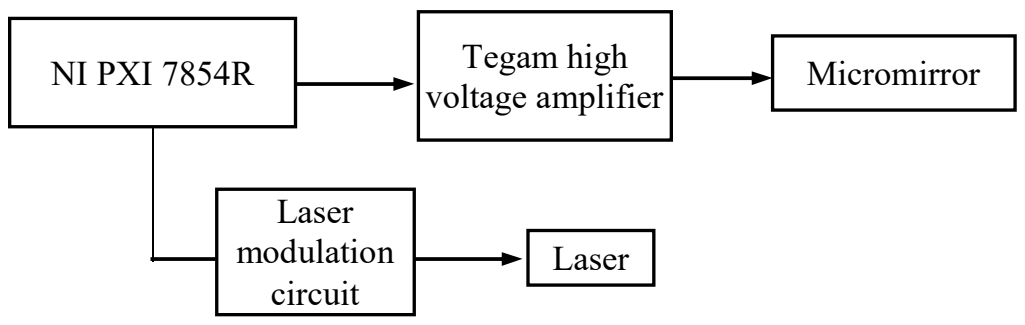

Fig. 6.5 The signal flow diagram.

The light efficiency of the display system setup on the optical bench shown in Fig. 4.6. It is not measured from the fabricated display module since it is difficult to position the detector between the optical components of the integrated display module in Fig. 6.4. Also since the same micromirror and the optical components are used for both system, the light efficiency in percentage should be consistent for both display system. The laser power attenuation in percentage is shown in Fig. 6.6 below. It is assumed that the input power of the laser beam is $100 \%$ at the micromirror. The laser power reduces to $28.5 \%$ after it is reflected by the micromirror. This is mainly caused 
by poor surface quality of the micromirror plate which is determined by the fabrication process, i.e., surface micromachining. The surface micromachining requires releasing holes and dimples on the micromirror plate [112]. As a result, the reflected laser beam contains a lot of light noise i.e., small laser dots, as shown in Fig.6.7. The lens further reduces the laser power about $1.5 \%$. This is due to the fact that the light noise contained in the reflected beam are significantly diverged from the main beam owing to the amplification of the double concave lens. The reflected laser beams before and after going through the double concave lens are shown in Fig. 6.7. It can be observed that the clustered small dots are spreading out after the double concave lens. When measuring the power, only the center beam is measured which leads to the decrease of the laser power measurement after the concave lens. It is noted that in the real application, the pinhole which is used to limit the laser beam size will further reduce the light efficiency.

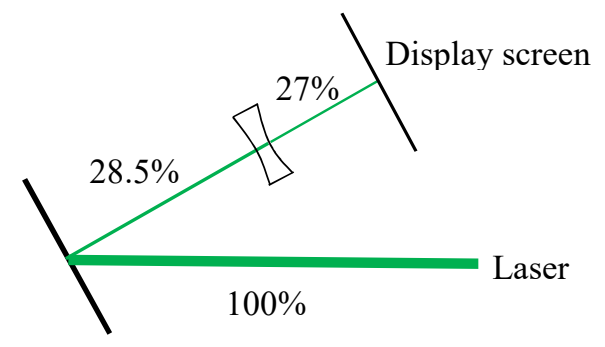

Fig. 6.6 Laser efficiency of the display system.

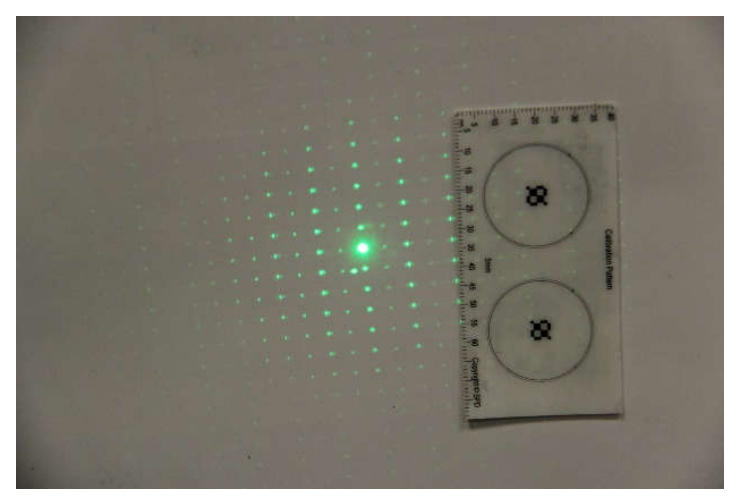

(a)

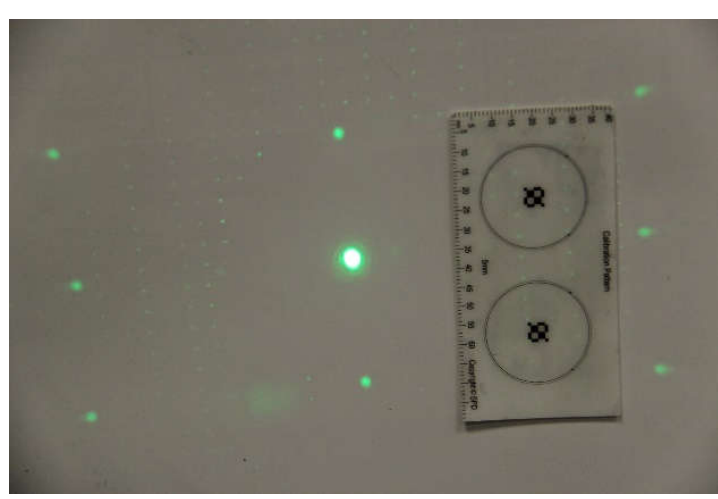

(b)

Fig. 6.7 (a) The reflected laser beam by the micromirror (b) The reflected laser beam after the double concave lens. 


\subsubsection{Real image and HUD setup}

The prototype of real image is shown in Fig. 6.8. A second hand car is cut in the middle and the part that has the windshield and the dash board is used to form the two configurations of the automotive HUD in lab environment. In order to clearly demonstrate the setup of the HUDs, the HUD is set up in the center area of the dashboard instead in the dashboard on the driver's side. The air condition module of car is removed in the center of the dashboard to make enough space for the HUDs. A rectangular hole is drilled through the dashboard to make optical path for the projection. Due to the relative large size of the display module, the display module is positioned under the dashboard instead of on it. A tailored cardboard is fixed at the bottom of the inside space of the dashboard which works as the base for display module. The display module is bond with an "L" shape stand which is made of a thin aluminum sheet which is flexible to be bend. It is used to adjust the pitch angle of the display module. The display module and the "L" shape stand are positioned on the base cardboard. When installing the display module and adjusting its position, the display need to be turned on so that the position of the image on the windshield can be observed in real time. A white paper is attached to the outside of the windshield and covers the whole projection area, so that the projection image can be observed while adjusting the position of display module. Also the mirror-reflective film is attached to the inside of the windshield in the same area to make sure the reflected laser beam is directed to the viewer in front of the film. The reflected light will enhance the brightness on the diffuse-reflective film that will be stacked on the mirrorreflective film. By adjusting the horizontal position and pitch angle of the display module, a best gesture of the display module can be found at which the whole projected pattern can go through the rectangular hole and be observed on the white paper and the projected light will be reflected to viewer in front of the projection films. Then the diffuse-reflective film which has the same size as the mirror-reflective film is bonded on top of it. As a result, a bright display can be observed if looking in front of the coated films. A projector is used to generate a background image to simulate the views on the road. The vector pattern designed in chapter 5 is display in $40 \mathrm{~Hz}$ refreshing rate and the display result is shown in Fig. 6.8 and Fig. 6.9. It can be seen that the real image of the display has a large viewing angle. 


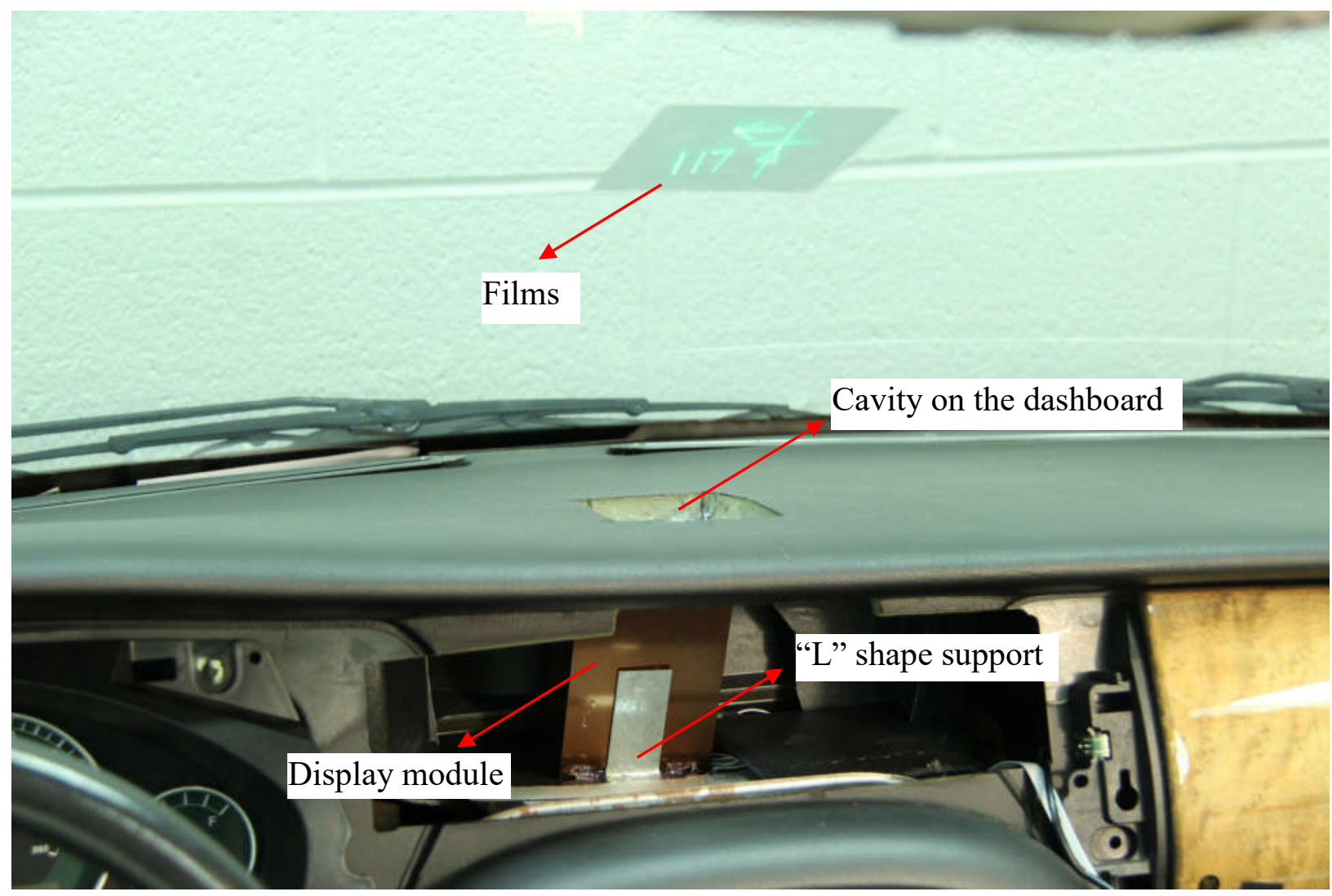

Fig. 6.8 Prototype of the real image HUD.

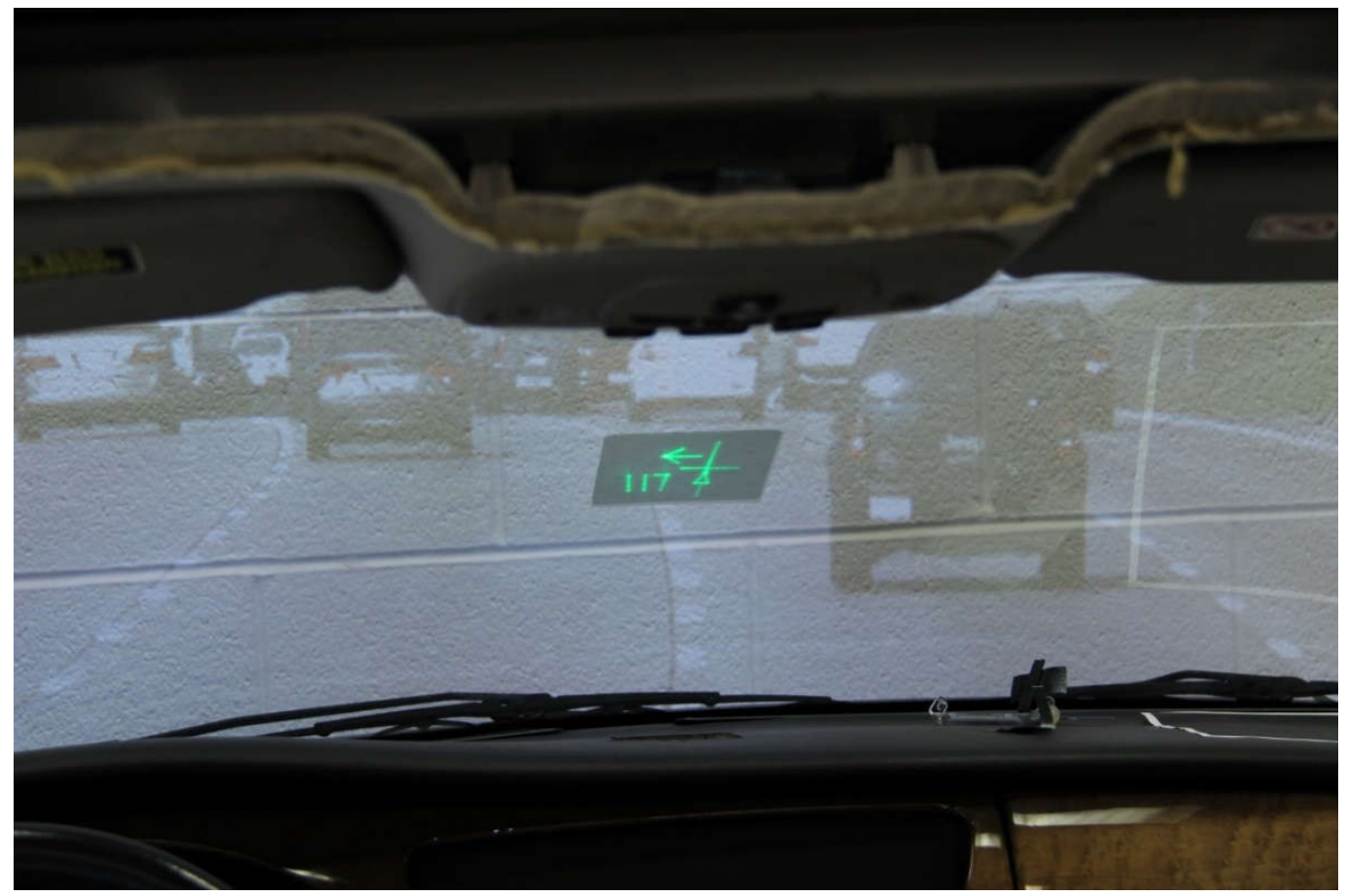

Fig. 6.9 Real image display result. 


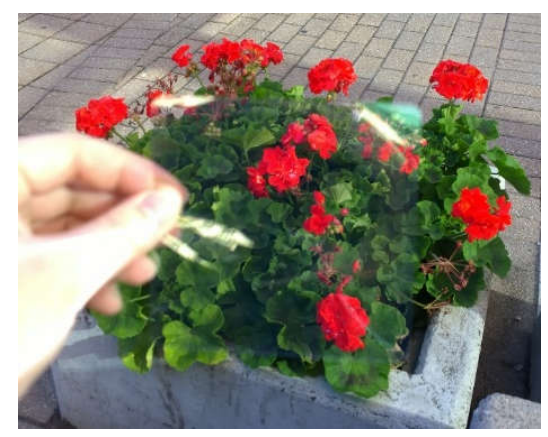

(a)

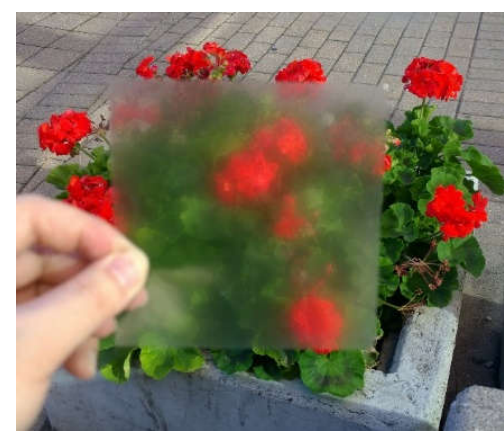

(b)

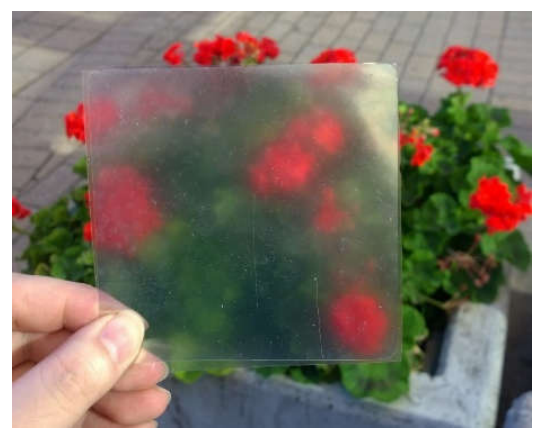

(c)

Fig. 6.10 Display films (a) Mirror-reflective film (b) Diffuse-reflective film (c) Diffuse-reflective film attacked on top of the mirror-reflective film.

The used mirror-reflective film is from Garmin and is widely used for LCD based automotive HUD. The diffuse-reflective film is the anti-glare coating film for the mobile devices. The transparency of both films are tested in the outdoor environment as shown in Fig. 6.10. The two films are separately tested for their transparency first (shown in (a) and (b)). It is can be seen that the mirror-reflective film is much more transparent than the diffuse-reflective film. The transparency of the two films with the diffuse-reflective film stacked on of the reflective film is exhibited in (c). It can be concluded that the current selected defuse-reflective is the main barrier for the transparency of the display films. Still the current diffuse-reflective film is already the best among serval diffuse-reflective films that are tested for the presented real image HUD setup. Usually there is a tradeoff between the transparency and the ability of diffusing the light of the diffuse-reflective film. More research needs to be conducted as the further work to develop a defuse-reflective that is suitable for the real image HUD setup presented in this section.

\subsubsection{Virtual image HUD setup}

The virtual image HUD utilize the same experimental car as the real image HUD. The setup is shown in Fig. 6.11. Similar to the real image HUD a cardboard is used as the base for the whole setup. A metallic coated fold mirror is bonded on an "L" shape flexible aluminum sheet which can adjust its pitch angle. A small display screen is mounted on the inside of one edge of the cavity which is opposite to the fold mirror as shown in Fig. 6.11. The display screen uses the material used for the projection screen which is highly diffuse reflective. A HUD reflector which has the similar characteristic with the mirror-reflective film is positioned on top of the dashboard above the cavity. Two small "L" shape flexible stands are attached to the reflector to adjust its pitch angle. 
The pitch angle of the fold mirror, display screen and the HUD reflector are needed to be adjusted corporately to make the display pattern visible to the viewer in front of the HUD reflector. The display image is shown in Fig. 6.13. It can be seen that in addition to the vector graphics the projection screen can also be seen in the virtual image. This is due to the fact that the background light i.e., the interior illumination of the room, is relatively strong and illuminated on the projection screen. This can be solved by installing a unidirectional glass on the cavity so that the outside light cannot be illuminated on the projection screen. Also the whole HUD system needs to be properly sealed to prevent the interference of the background light.

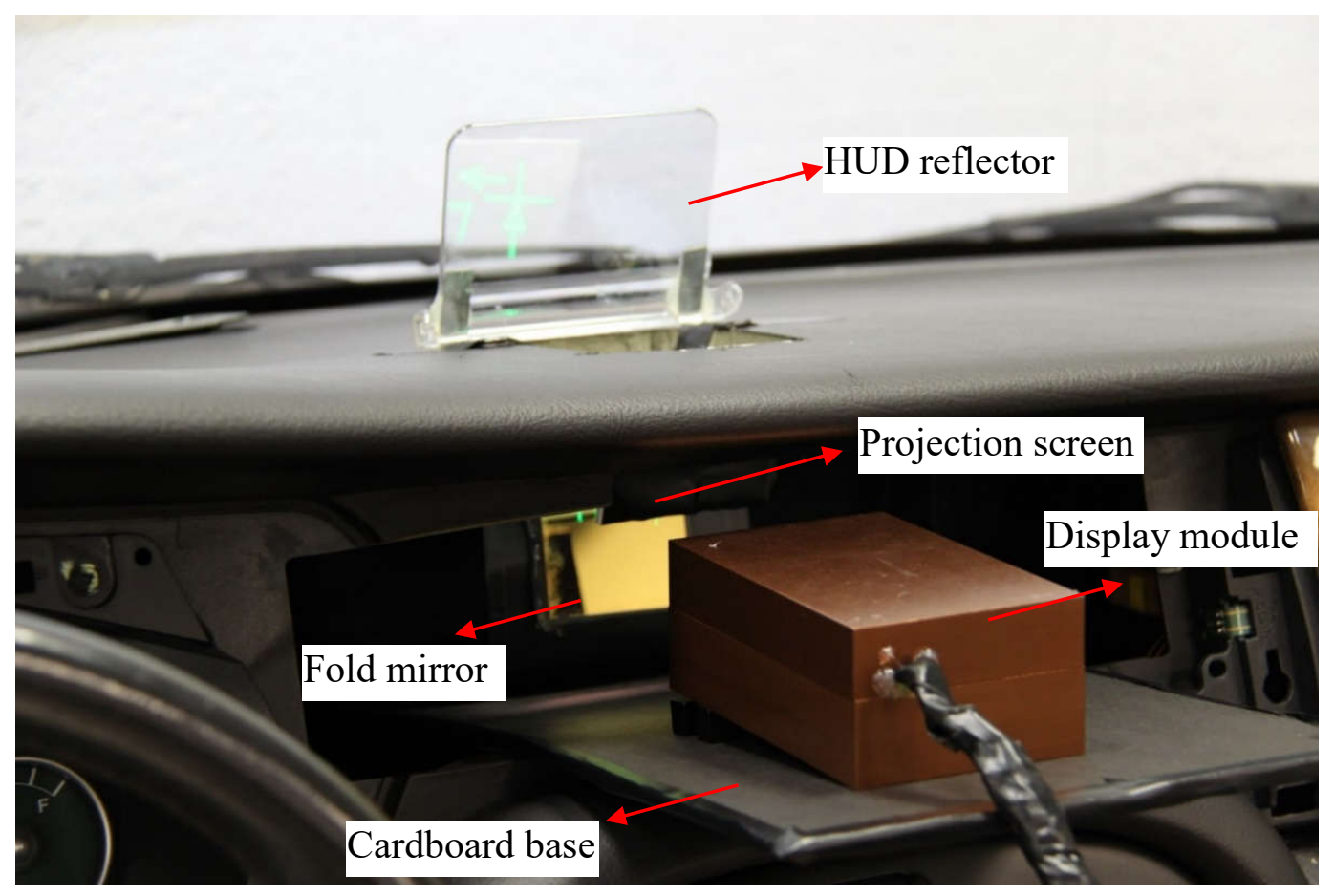

Fig. 6.11 The prototype of the virtual image HUD.

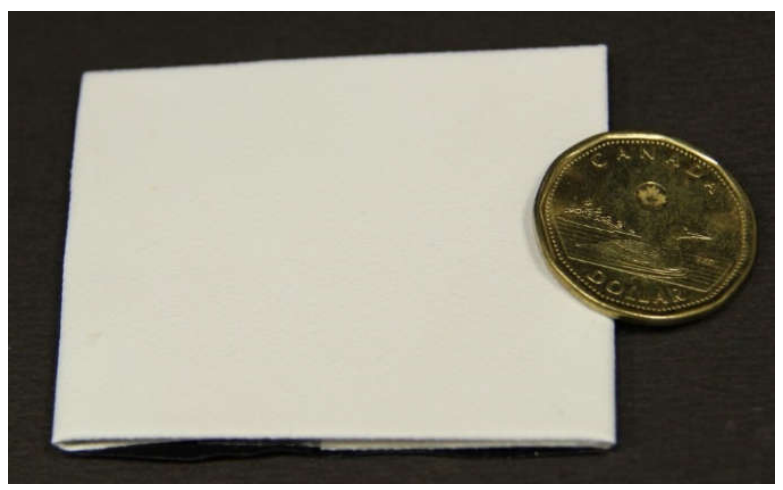

Fig. 6.12 The projection screen used for virtual HUD. 


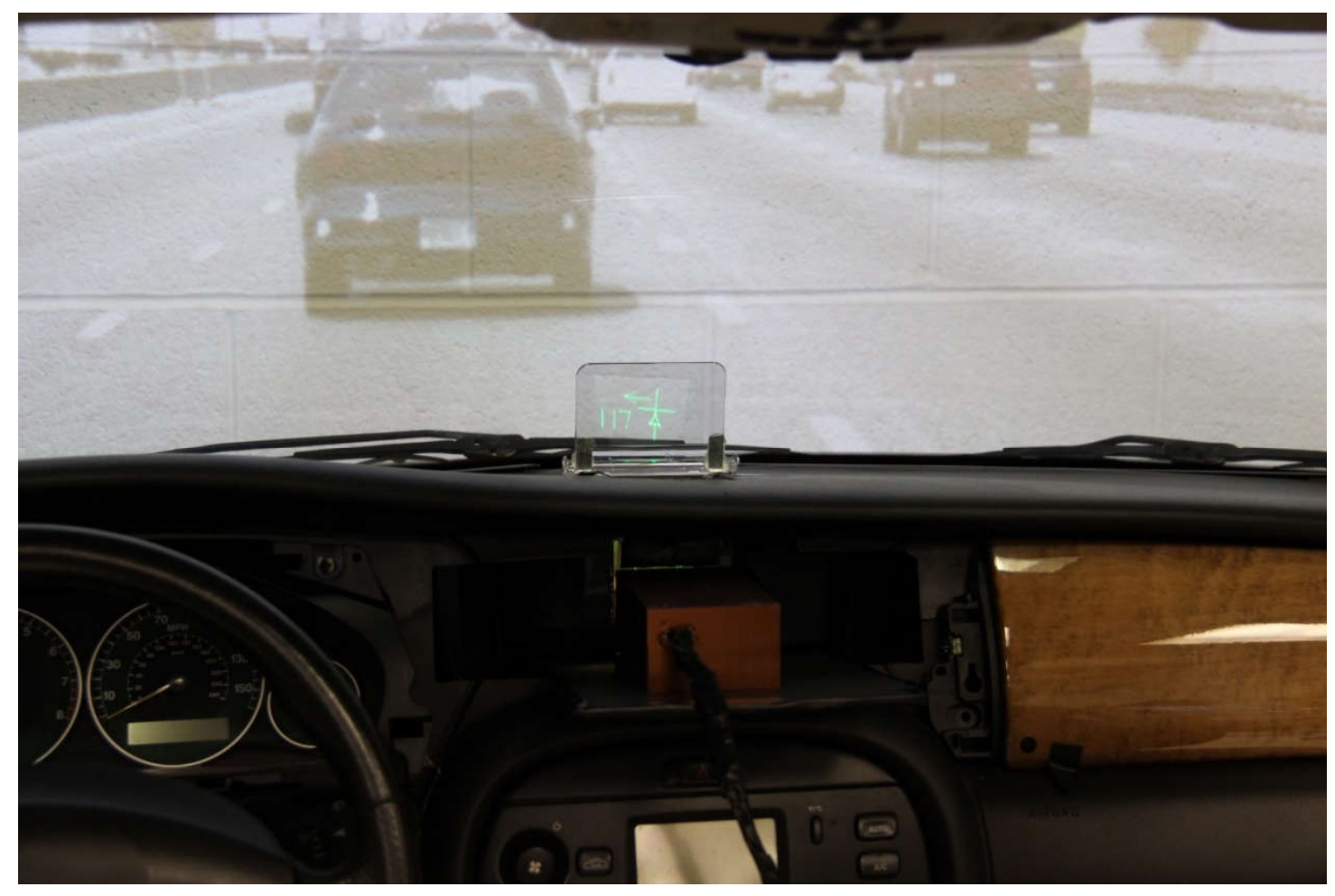

(a)

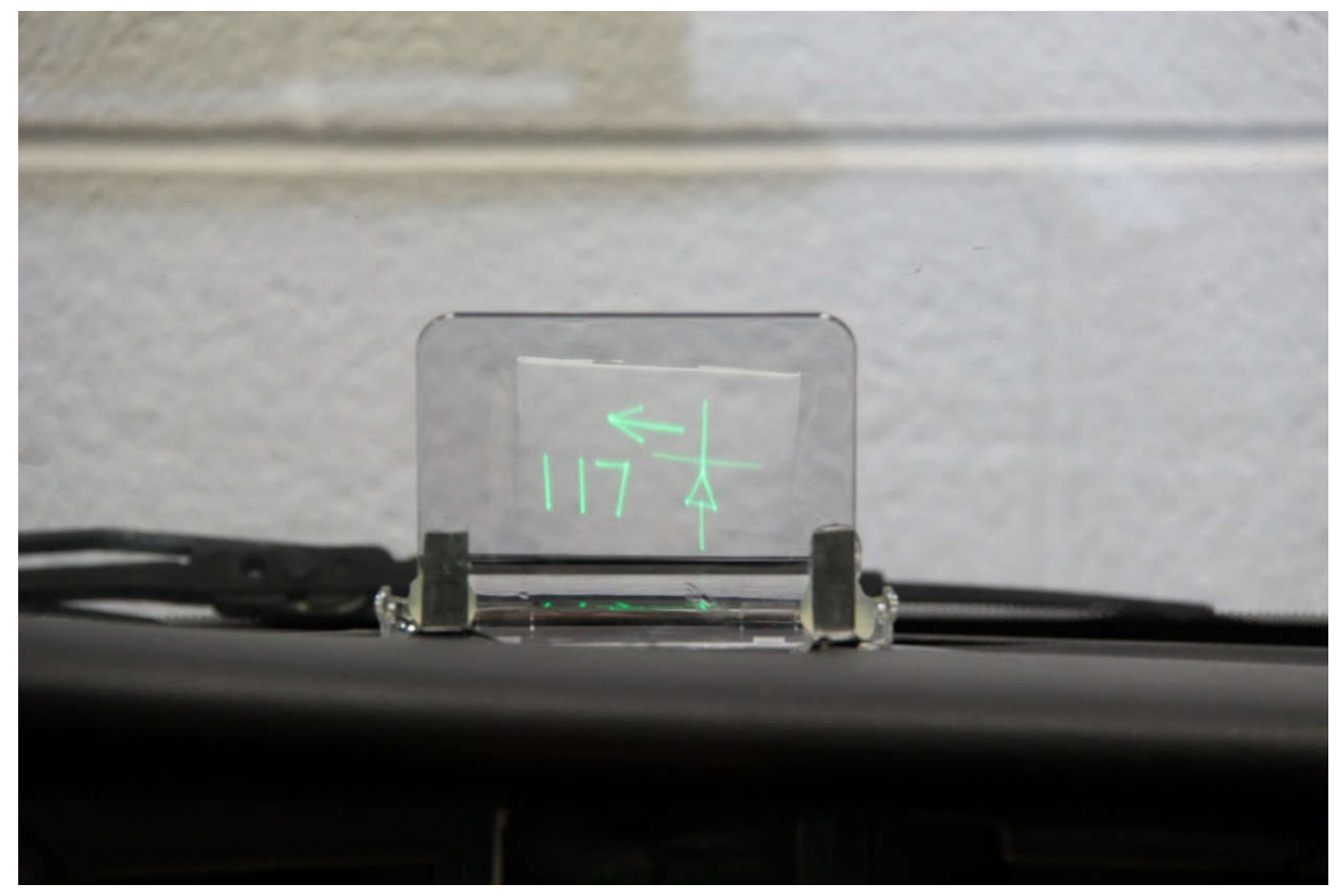

(b)

Fig. 6.13 Display result of the virtual image HUD. 


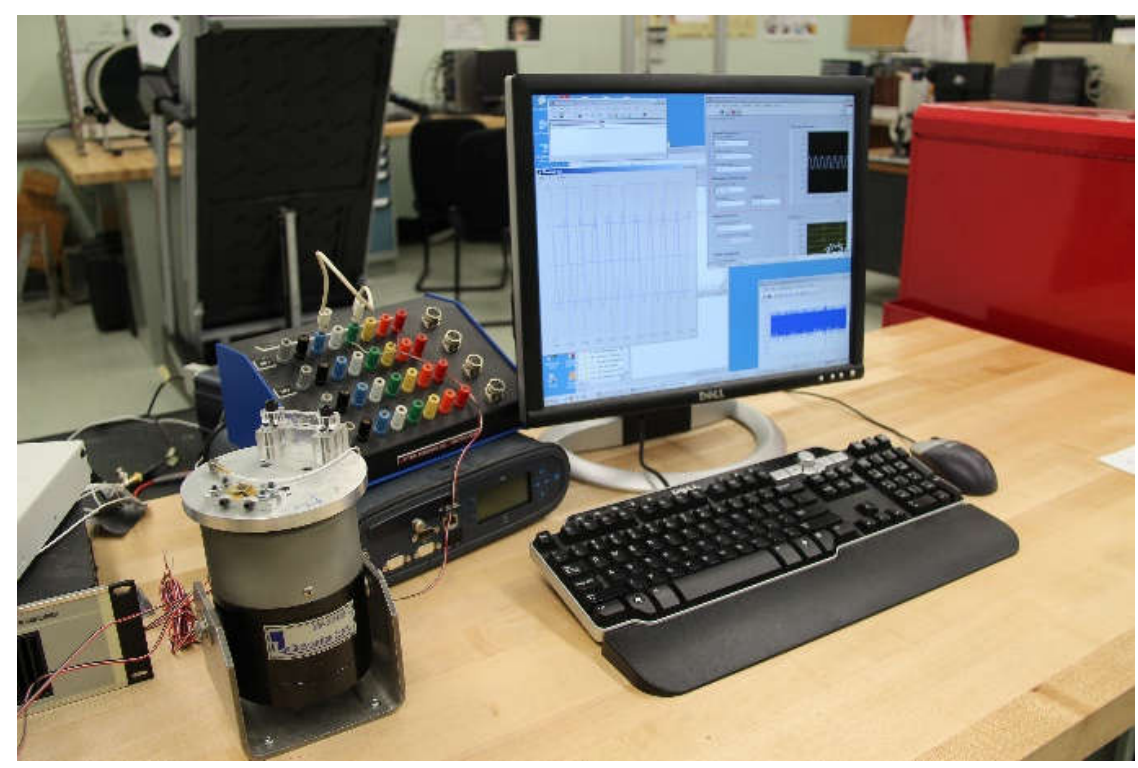

(a)

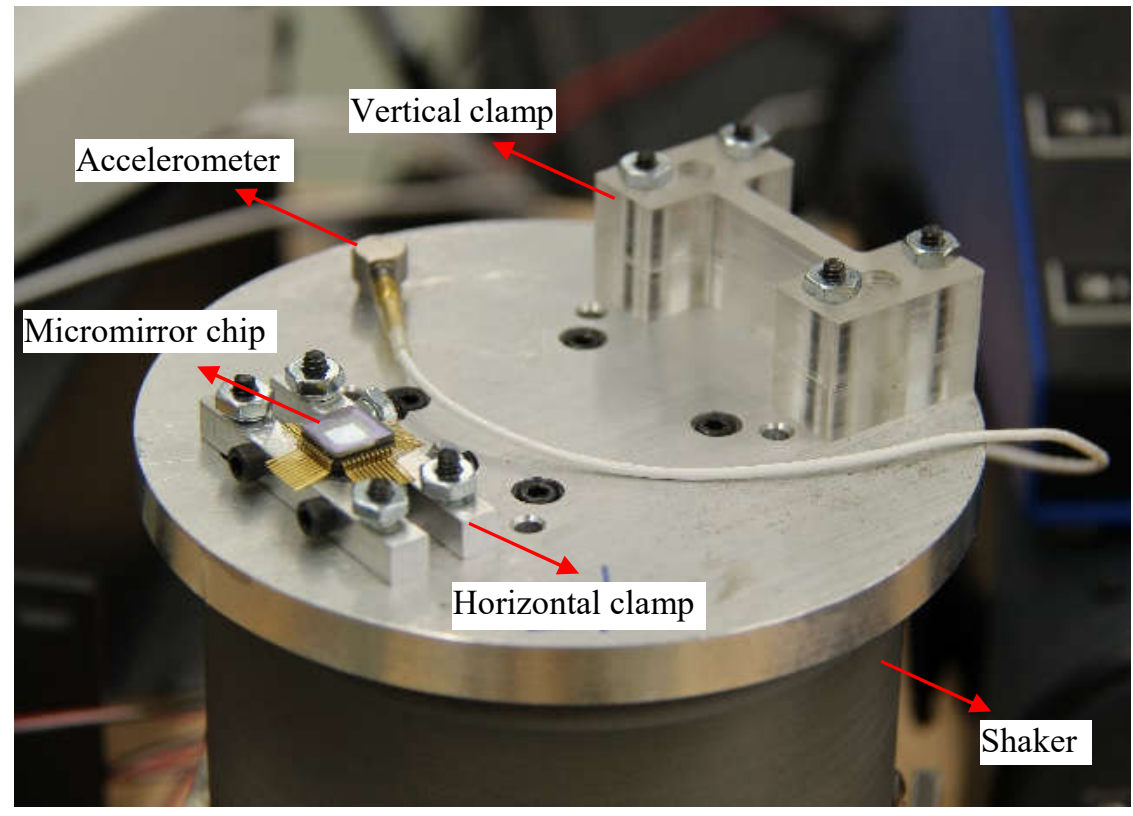

(b)

Fig. 6.14 (a) Micromirror vibration test system (b) Setup on the shaker.

A vibration test has been performed to test the reliability of the micromirror. An aluminum circular plate with two clamp fixtures is used to fix the micromirror and connect with a shaker as shown in Fig. 6.14. There are two fixtures on the circular plate which are used to fix the micromirror horizontally and vertically so that the reliability tests can be performed on two orthogonal directions of the micromirror. There is a small aluminum connector that is glued on the 
backside of the micromirror chip using epoxy adhesive which is a solid connection and has high strength. In the test, the aluminum connector is clamped on the horizontal and vertical clamps and most of the vibration can be conducted on the tested micromirror. The shaker is driven by sinusoidal signals which will drive the shaker to have a sinusoidal acceleration. A piezo accelerometer is screwed on the circular plate so that the real time acceleration can be captured and monitored from a computer. Before the test, the micromirror is placed in the display system (similar to the one shown in Fig. 4.6) to scan some straight lines. The scanning range of the micromirror for each line is manually marked on the display screen. For each test, the sinusoidal acceleration is maintained about $30 \mathrm{~s}$ then the micromirror is taken off from the clamp and positioned in the display system to scan the same straight lines that is previously marked on the display screen. If the micromirror after the acceleration test is still able to scan the same lines, it is then tested for higher acceleration. The same acceleration is performed on two orthogonal directions of the micromirror. The peak value of the acceleration is recorded for each test and the maximum acceleration applied is $5 \mathrm{G}$ under which the micromirror is still properly functional.

\subsection{Compact design of display module}

The size of the display module is important for the automotive HUD application due to the limited space on the dashboard or under it. The prototype presented in the previous section is still relatively large and as a result, in real image HUD setup there is not enough space to place it on the dashboard; in virtual image HUD setup the display module has to extend beyond the space under the dashboard. A more compact display module is developed in this section.

The compact design of the display module is shown in Fig. 6.15 which is composed of two fold mirrors, a laser diode module, a double concave lens, a micromirror and fixtures for the optical components. In order to save the space, the laser is placed on the base plate with its longitudinal direction parallel with the longitudinal direction of the base plate. A fold mirror is used to direct the laser beam to the micromirror. The fold mirror is mounted on a fixture which is able to rotate about the axis normal to the base plate. The micromirror is soldered on a printed circuit board which is mounted on an aluminum slider. The vertical position of the micromirror can be adjusted by sliding the slider along a slide track. The slide track can also rotate along a vertical axis. The reflected laser is then directed by a second fold mirror. The double concave lens is fixed in a lens

holder which can move vertically in a slide track. The lens slide track and the fold mirror holder 
can move along two tracks on the base plate so that the distance from the micromirror to the concave lens can be adjusted to obtain a sharp image. An enclosure is also designed as shown in Fig. (b). The overall dimension of the compact display module is $84 \mathrm{~mm} \times 40 \mathrm{~mm} \times 35 \mathrm{~mm}$ which is about only $36 \%$ of the prototype of the display module presented in the above section. The display result is shown in Fig. 6.16.

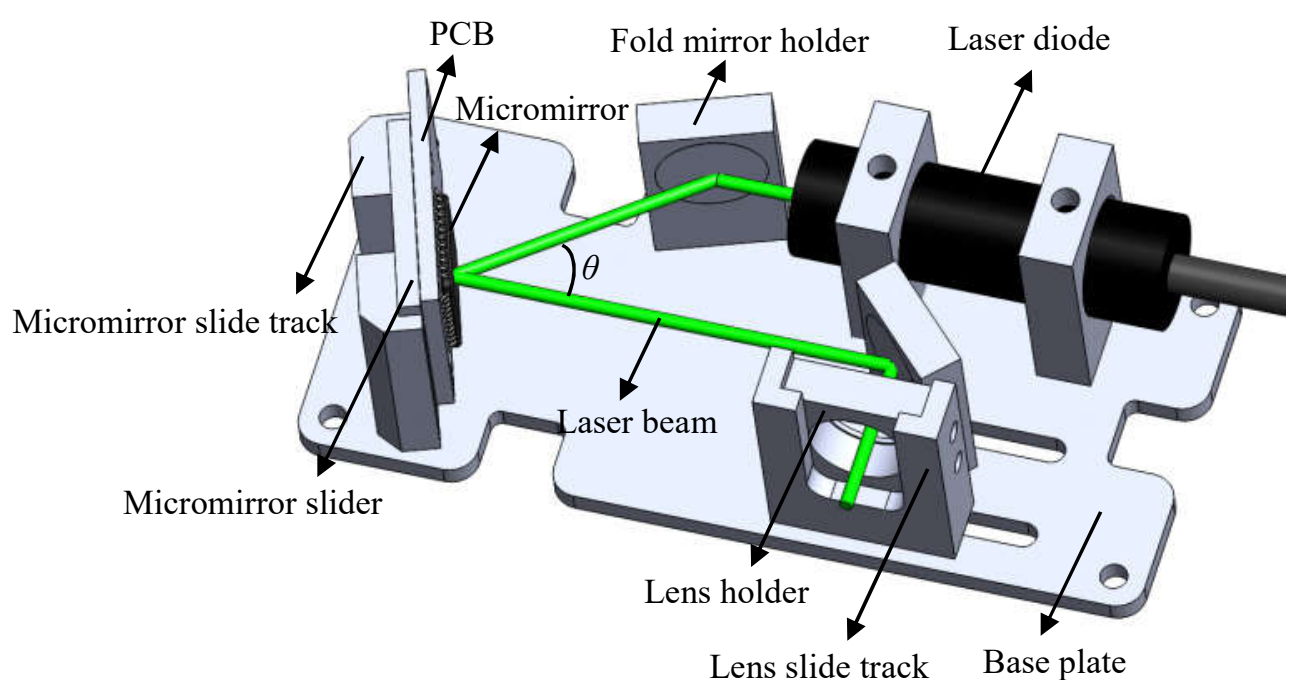

(a)

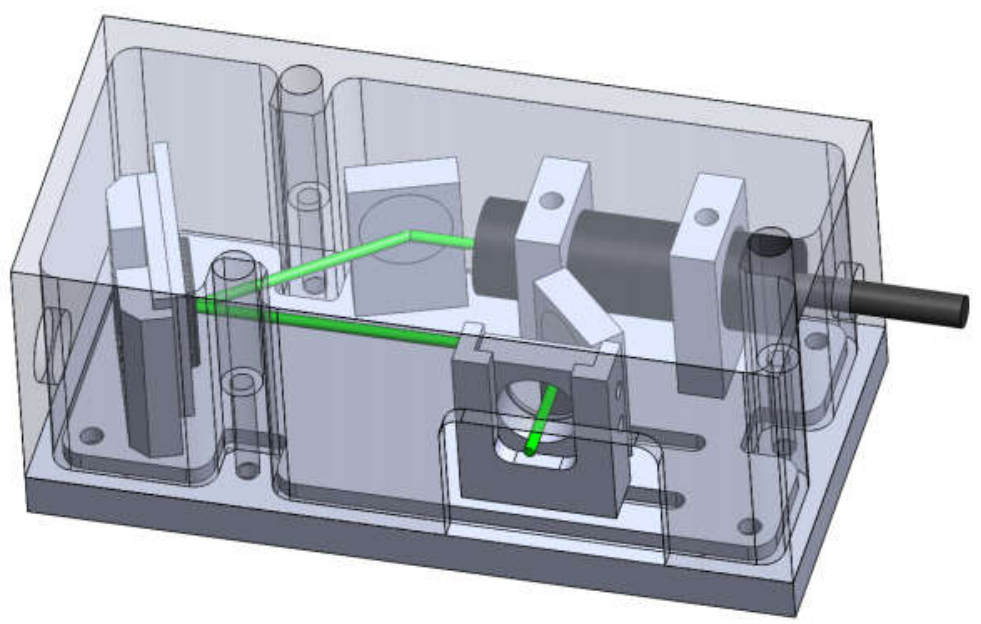

(b) 


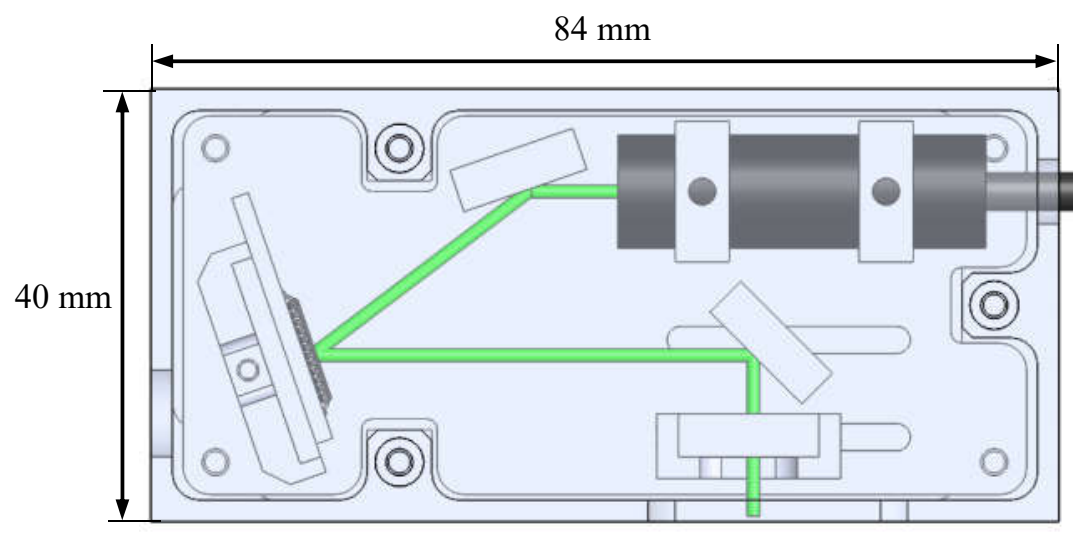

(c)

Fig. 6.15 Compact structure design of the micromirror.

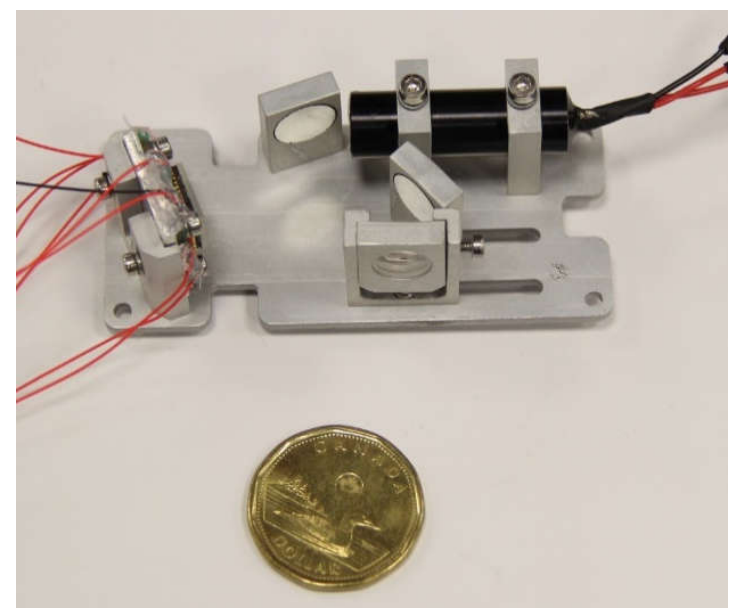

(a)

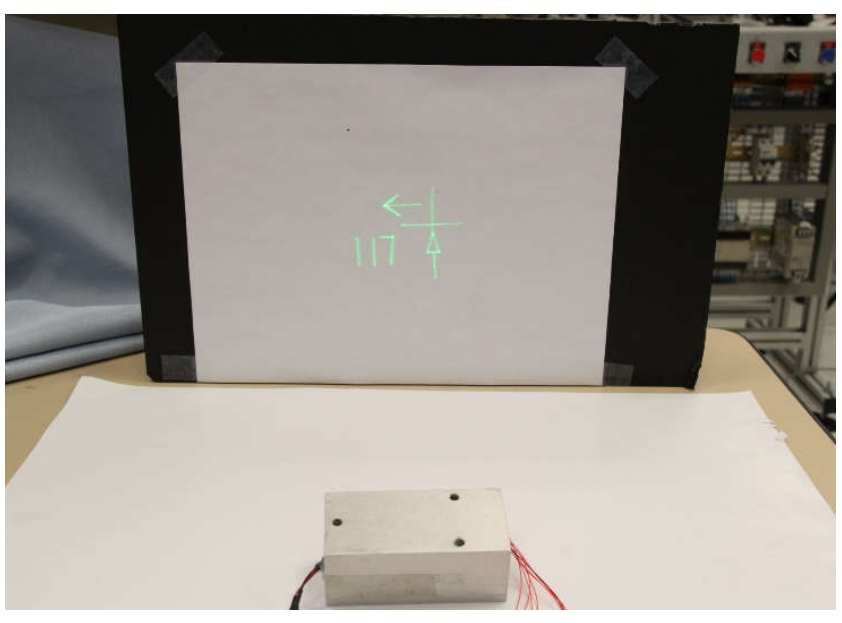

(b)

Fig. 6.16 (a) Prototype of the compact display module. (b) Display result of display module The compact display module is used to construct a real image HUD as shown in Fig. 6.17. The display module is positioned on the dashboard attributed to its small volume. It can be used as a aftermarket product which can be easily installed by the customer. It is noted that the diffusereflective film is also improved with higher transparency than the one used above. The comparison of the transparency is shown in Fig. 6.18. The viewing angle of the constructed HUD is tested to be about $\pm 60^{\circ}$. 


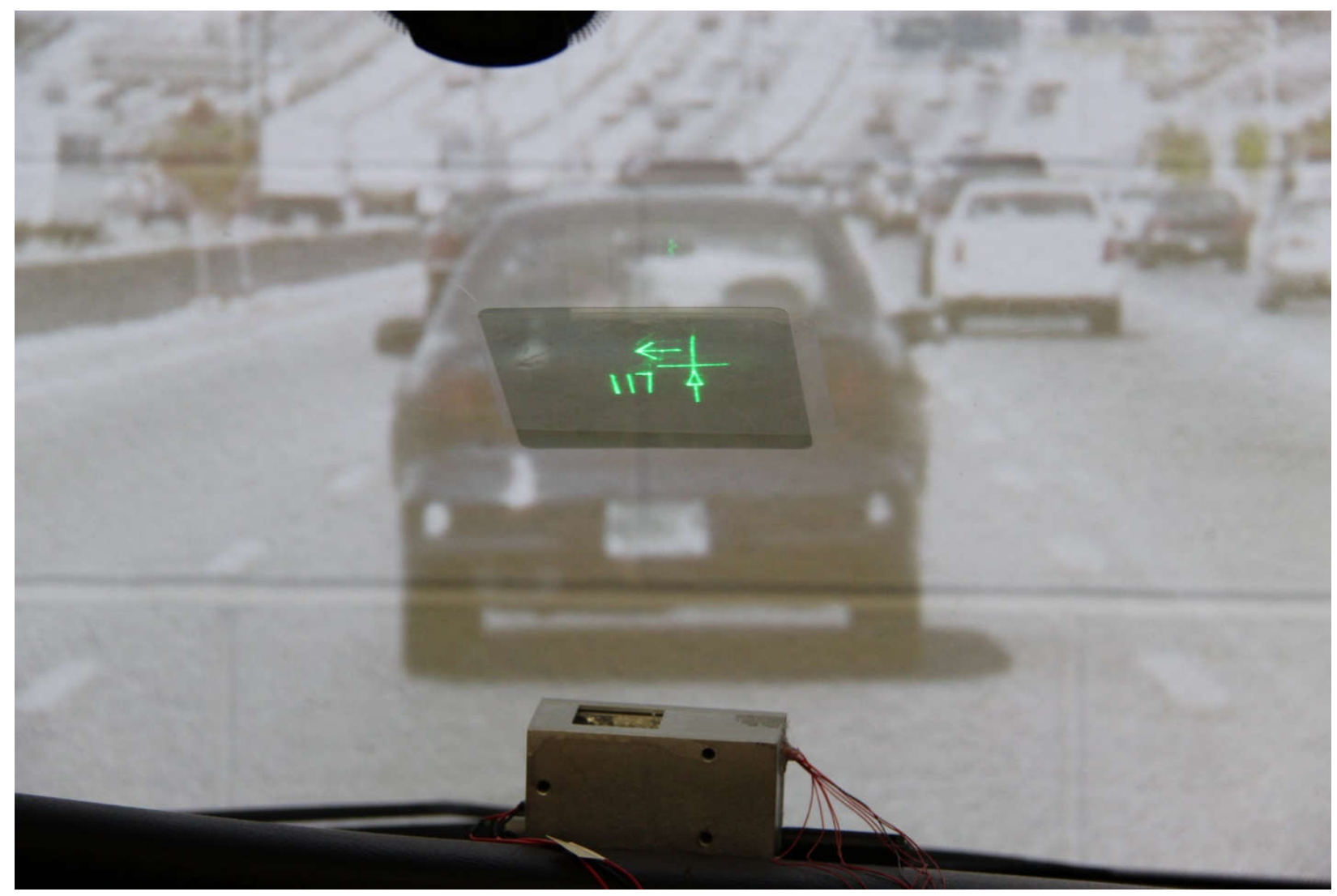

Fig. 6.17 Real image HUD of compact display module.

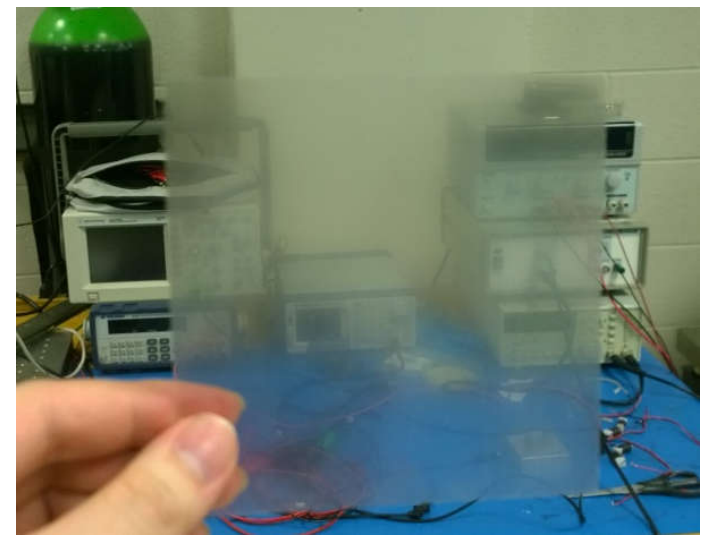

(a)

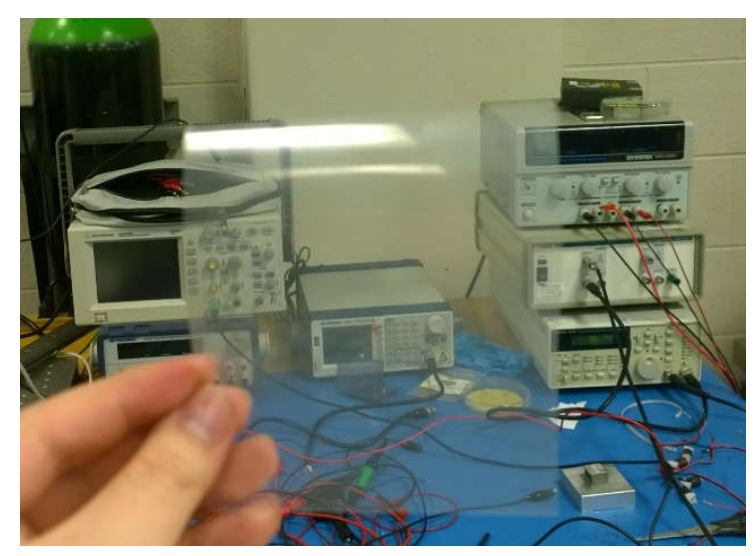

(b)

Fig. 6.18 Comparison of the diffuse-reflective films.

The visibility of the real image HUD has been tested in the lab environment. The experiment setup is shown in Fig. 6.19. Two high power light with their power adjustable are used as the external light source. In the experiment, the display result is recorded using the camera for various illuminance from 0 to 15000 Lux outside of the windshield. The illuminance is measured using a 
lux meter from the outside of the windshield right above the display pattern. It has been observed that the display results starts to be washed away when the external result is higher than 6800 Lux. Some examples of the display results under different external illumination is shown in Fig. 6.20.

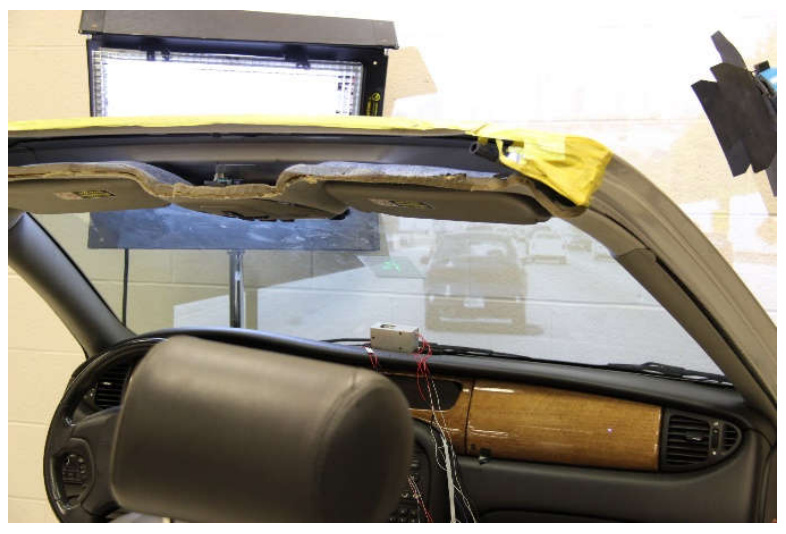

(a)

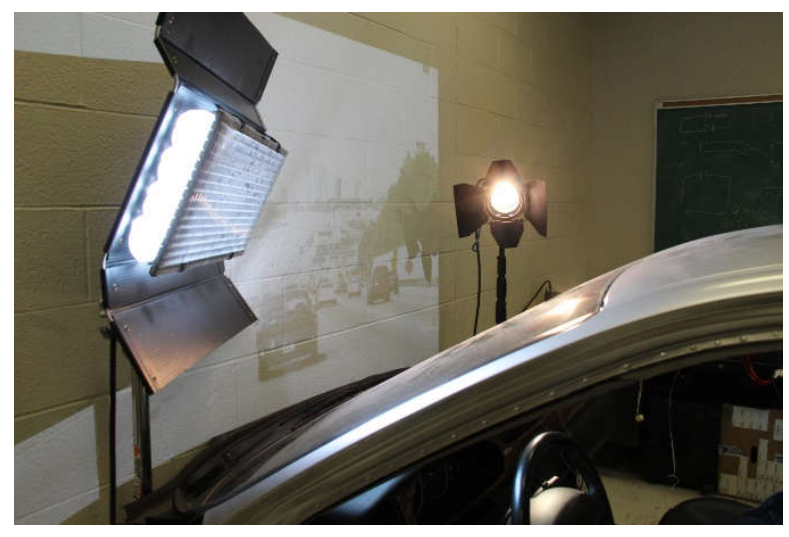

(b)

Fig. 6.19 Visibility test setup (a) Front view (b) Side view

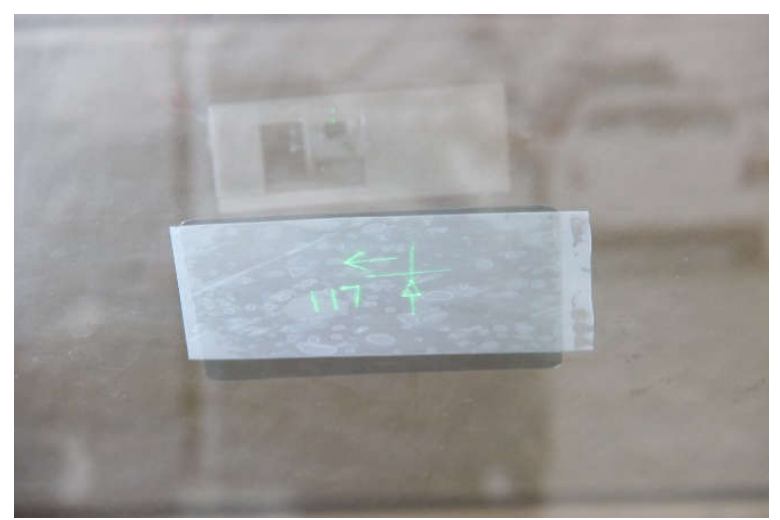

(a)

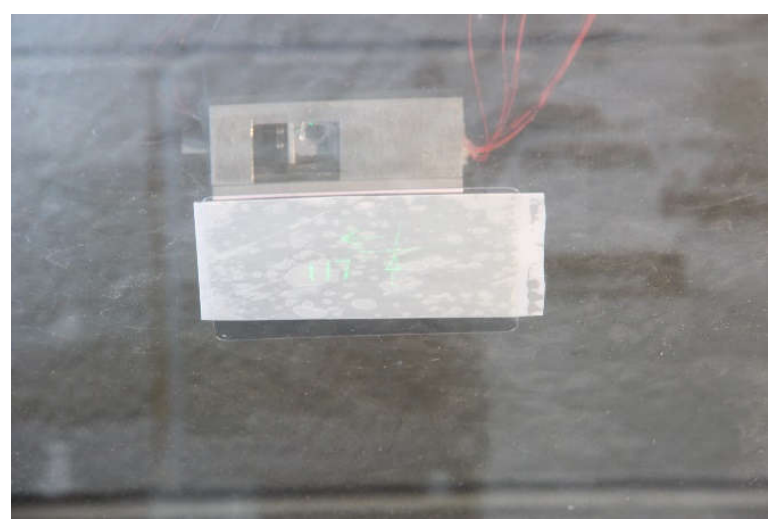

(b)

Fig. 6.20 Display results under different external illumination (a) 2300 Lux (b) 6800 Lux

\subsection{Summary and comparison with the other HUDs}

The developed HUD has a small profile which enables it not only to be originally integrated with the car but also installed as an aftermarket device on the dashboard. For example the dimensions of the developed compact display module is about $8.4 \times 4.0 \times 3.5 \mathrm{~cm}$ which is smaller in length and width direction than most of the commercial HUD modules that can be positioned on top of the dashboard, e.g., 1) The on-dashboard HUD display module from Garmin has a dimension of $10.8 \times 8.8 \times 1.9 \mathrm{~cm} \mathrm{2}$ ) The on-dashboard HUD display module from Navdy is 
claimed to have a size of $14 \times 13 \times 9.5 \mathrm{~cm} 3$ ) The NavGate suspension type HUD display module from Pioneer has a size of $20 \times 19 \times 12 \mathrm{~cm}$. It is noted that the height of the developed compact display module is currently limited by the size of the micromirror packaging and the size of its PCB adapter, both of which can be further significantly reduced. The length and width dimension can also be further reduced by using laser diode instead of commercial laser diode modules. Comparing to the conventional display modules which are buried under the dashboard, the advantage is even more obvious. The display module for the conventional HUD (virtual image HUD) should be at least twice the size of the displayed virtual image. In this case, in order to display the same image size ( 5 inch) as the real image HUD presented above, the display module should be at least has a dimension of $18 \times 18 \mathrm{~cm}$ (height is not considered). Furthermore, the developed real image HUD has a simple structure which only requires the projection film and the display module which enables it to be easily installed after the manufacture.

The developed HUD system has a much lower cost than the currently available ones due to the low cost of the micromirror and the simple structure of the optical setup. Since the micromirror requires only two thin layers, it can be fabricated using commercial fabrication process, i.e., PolyMUMPs which leads to good fabrication yield and low cost. It has been estimated by the MEMSCAP the PolyMUMPs provider that the cost of a single micromirror is less than 5 dollar for mass production. Due to the simple structure, the display module will have a relative low cost (less than 20 dollars). However, for the LCD, DMD and LCoS based HUD, a single display generation unit costs more than 20 dollars without considering other optical components required in the display module. For example, the TI DMD micromirror array DLP3000Q1 which is automotive qualified chip cost more than 100 dollars even with large purchase quantities. Also the developed real image HUD has very large viewing angle, i.e. $\pm 60^{\circ}$, compared to that of the virtual image HUD, i.e., $5^{\circ}$ [146], which is the main HUD setup of the products on the market. 


\section{Chapter 7 Conclusions and future work}

\subsection{Conclusions and contributions}

(1) Developed two novel electrostatic out-of-plane microactuators

Two novel actuators, i.e., two-row-finger (TRF) and two-width-finger (TWF) electrostatic repulsive-force microactuators have been developed in Chapter 2 and Chapter 3. Both actuators have been fabricated using PolyMUMPs and tested. The measured results show that the TRF actuator is able to achieve $11.5^{\circ}$ mechanical rotation and the ORF actuator is able to achieve $7.6^{\circ}$ rotation under $150 \mathrm{~V}$ DC driving voltage. Two mathematical models have been developed for them respectively, which combine the analytical derivations and numerical simulations. The models are able to evaluate the rotation angle variations with the change of driving voltages. The calculated results from the model match well with the testing results. The discrepancies between the model and the test results are approximately $8 \%$ and $11.8 \%$ at $150 \mathrm{~V}$ driving voltage for the TRF and TWF actuators respectively. The models can be used to predict the rotation angles of the two actuators, which can save significant time than numerical simulations. The methodology used in developing the model for these two actuators can be used to build models for other electrostatic actuators. It has been proved that both actuators can achieve much larger rotation angle than that of the previously reported repulsive-force actuators of the same actuator size, spring stiffness and driving voltage. The rotation angle improvements of these two actuator are about $100 \%$ and $33 \%$ at $150 \mathrm{~V}$ respectively.

Besides of the advantage of lager rotation angle, these two actuators do not suffer from the "pull-in" instability associated with the conventional electrostatic actuators. In addition, these two actuators require only two thin layers which can be easily fabricated using mature and commercially available surface micromachining process. The successful development of the two repulsive-force actuators and their models meet the objective 1 of the thesis.

(2) Developed a 2D scanning micromirror

A 2D scanning micromirror has been developed in Chapter 4 based on the novel TRF actuator. A vector scanning display system has been built using this micromirror. Both static and dynamic performances are tested. It has been proved that the micromirror has much larger rotation angle (more than twice) and faster response speed than those of the ORF micromirror which has 
approximately the same size and spring stiffnesses. The development of the micromirror meets the first part of the objective 2 of the thesis.

(3) Developed an advanced display approach

An advanced display approach has been developed in Chapter 5 which is able to display laser patterns with less distortion and higher refreshing frequency compared to the previously developed generic display approach. The advanced display approach includes steps of the camera based calibration, point distributing and voltage allocating. All the steps are easy to be implemented in both the micromirrors developed in this thesis and previously. The experimental tests have been conducted to verify the advanced display approach. The successful development of the advanced display approach meets the second part of objective 2 of the thesis.

(4) Developed an automotive HUD system

Two display modules have been designed and fabricated, which integrates the laser, the micromirror, and the optical components. One of them is more compact than the other. The display modules have been used to form two configurations of the automotive HUD, i.e., real image and virtual image HUDs. Both HUDs are installed in a testing car. Experimental results show that the HUDs can generate display patterns with good quality in both configurations. The development of the automotive HUD has met the objective 3 of the thesis.

\subsection{Future work}

\section{(1) Actuators}

The models have been developed for the TRF and TWF microactuators in the thesis which can predict the rotation angles of the actuators giving all the dimensions of the actuators and the corresponding driving voltages. Furthermore, optimization models can be developed in the future to optimize some dimensions of the actuator. In the TRF actuator, the dimensions that can be optimized include the finger width, the length of the lower and upper fingers and the upper spring stiffness. For the TWF actuator, the dimension of the finger widths of the two-width finger can be optimized.

Another future work that can potentially improve the dynamic performance of the actuator is to incorporate attractive driving force in the actuators. The repulsive-force actuators developed in this thesis has a unidirectional driving force, i.e., electrostatic out-of-plane repulsive force. As a 
result, the actuator's moving part relies on the mechanical restoring force to be pulled to its initial position. It is possible that attractive force can be added to pull the moving part of the actuator back to its initial position. This can be done by adding extra electrodes to form parallel-plate or vertical comb-drive type actuators. As a result, the bidirectional actuation force based actuator has a faster response speed.

\section{(2) Micromirror}

The micromirror presented in the thesis is fabricated using surface micromachining process which has releasing holes and dimples on the micromirror plate. Consequently, the micromirror plate has a relatively poor surface quality which reduces the light efficiency. A potential solution is to bond a metal coated SOI mirror plate which has the good surface quality on top of the micromirror. More researches need to be done to develop such a bonding process to improve the surface quality of the micromirror without sacrificing too much response speed.

\section{(3) HUD}

It has been mentioned in Chapter 6 of the thesis that the display films for the real image HUD configuration is of low transparency. With the development of the laser scanning display technologies a projection film to be used for real image HUD is highly desired and worth further research. One possible solution is to dope some small particles into the transparent film which can make the film diffuse and reflective. The doped film can be bonded with a film or a glass which is embedded with microlens.

The size of the display module and the HUD system can be further reduced by improving the integration level of system. For example, 1) Integrate the control and driving circuit with the display module; 2) Add a wireless communication module into the display module so that it can receive and display information from other mobile devices such as a cellphone. 3) Use the laser diode as the light source instead of a laser diode module which has a bulky volume. Meanwhile, the optical system design needs to be improved to obtain sharp displays. Also multiple micromirrors can be integrated together to display more information. Laser diodes with multiple colors can be integrated to generate colorful displays.

More reliability tests need to be performed according to the automotive industrial standards. The tests that are to be performed include the impact and vibration tolerance and temperature 
tolerance. Also the visibility of the displayed pattern with respect to the background illumination needs to be further studied in the outdoor environment. 


\section{Appendices}

\section{Appendix A}

The 2-D electric field simulation is to provide discrete data for the surface fitting to obtain $f_{\text {interdigitating_lower, }} f_{\text {interdigitating_upper }}$ and $f_{\text {non-interdigitating_upper, }}$ i.e., electrostatic force exerted on the rotation fingers along the cross section with unit length and unit driving voltage. $f_{\text {interdigitating_lower, }}$ $f_{\text {interdigitating_upper }}$ and $f_{\text {non-interdigitating_upper }}$ are functions of $h_{l}$ and $h_{u}$. The ranges of $h_{l}$ and $h_{u}$ should be wide enough to cover all possible values of vertical distance between fixed and rotation fingers. For a given actuator, according to Eqs. (2.5) to (2.8), the limits of $h_{l}$ and $h_{u}$ can be written as Eqs. $(\mathrm{A} 1) \sim(\mathrm{A} 4)$.

$$
\begin{aligned}
& 0 \leq h_{l_{-} \text {interdigitating }} \leq L_{\text {lower }} \sin \theta_{l_{-} \max }
\end{aligned}
$$

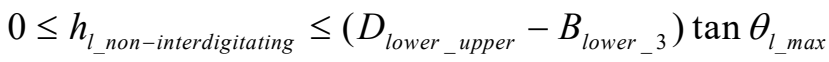

$$
\begin{aligned}
& 0 \leq h_{u_{-} \text {interdigitating }}<L_{\text {lower }} \tan \theta_{u_{-} \max } \\
& 0 \leq h_{u_{-} \text {non-interdigitating }} \leq\left(D_{\text {lower_upper }}-L_{\text {lower }} \cos \theta_{l_{-} \max }\right) \tan \theta_{u_{-} \max }
\end{aligned}
$$

$h_{l}$ interdigitating and $h_{l \_n o n-i n t e r d i g i t a t i n g}$ are representations of $h_{l}$ in the interdigitating area and non-

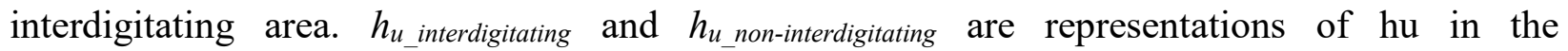
interdigitating area and non-interdigitating area. $\theta_{l_{-} \max }$ and $\theta_{u_{-} \max }$ are the maximum rotation angles of lower and upper rotation fingers which are achieved under maximum driving voltage. If $\theta_{l_{-} \max }$ and $\theta_{u \_ \text {max }}$ can be estimated, the range of $h_{l}$ and $h_{u}$, i.e., $h_{l \_ \text {interdigitating, }} h_{l \_n o n-i n t e r d i g i t a t i n g}, h_{u \_ \text {interdigitating, }}$

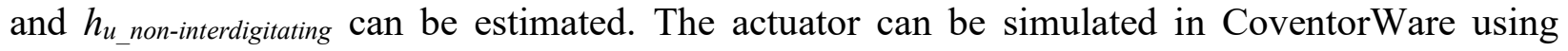
CosolveEM solver to perform coupled field simulation between mechanical and electrical domains. However, converged results cannot be obtained for a driving voltage higher than $25 \mathrm{~V}$ when using CosolveEM solver to simulate the present actuator due to the complexity of the electrodes of this actuator. Since $\theta_{l_{-} \max }$ and $\theta_{u_{-} \max }$ and then the ranges of $h_{l}$ and $h_{u}$ (according to Eqs. (2.5) to (2.8)) are obtained when the driving voltage is $150 \mathrm{~V}$, the coupled field simulation solver "CosolveEM" of Coventorware cannot be used. Instead, the following method is used to roughly estimate the maximum values of $\theta_{l_{-} \max }$ and $\theta_{u_{-} \max }$ and then the ranges of $h_{l}$ and $h_{u}$, which uses only electrical domain simulation instead of coupled field solver to obtain the torques generated by electric field which acts on the lower and upper rotation fingers of the actuator at different combinations of $\theta_{l}$ 
and $\theta_{u}$ under a unit driving voltage. For any other driving voltage, the resulting electrostatic torque equals to the square of the driving voltage multiplying the torque of unit driving voltage. Thus various curves of the torque acting on the lower rotation fingers, i.e., $T_{l o w e r}$ versus $\theta_{l}$ at different $\theta_{u}$ for any driving voltage can be obtained. One example is shown in Fig. A.1 (a) when the driving voltage is $150 \mathrm{~V}$. Similarly, various curves of the torques acting on the upper rotation fingers, $T_{\text {lower }}$ versus $\theta_{u}$ at different $\theta_{l}$ for any driving voltage can be obtained. One example of which is shown in Fig. A.1 (b) under $150 \mathrm{~V}$ driving voltage. The mechanical restoring torque generated by the lower springs versus its rotation angles is plotted as a straight line in Fig. A.1 (a). The mechanical restoring torque generated by the upper springs versus its rotation angles is plotted as a straight line in Fig. A.1 (b).

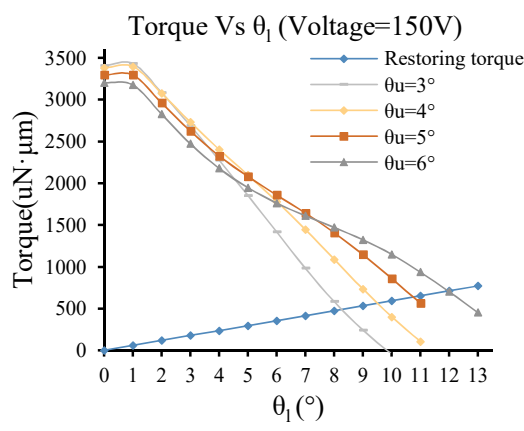

(a)

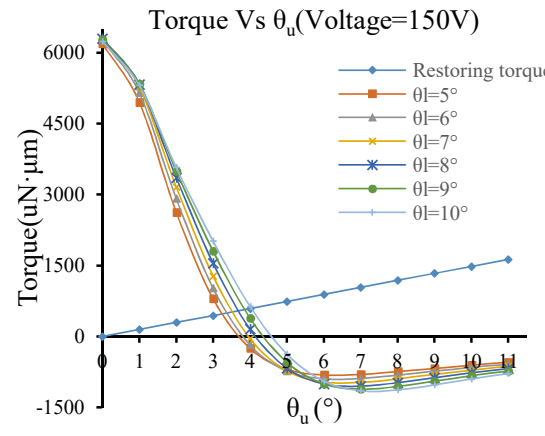

(b)

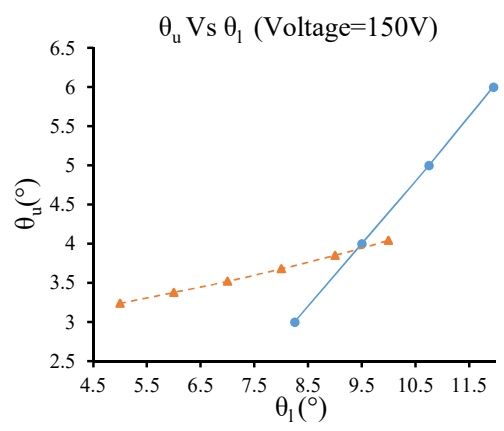

(c)

Fig. A.1 One example (driving voltage of $150 \mathrm{~V}$ ) determining the rotation angles of lower and upper rotation fingers graphically. (a) Torques acting on lower rotation fingers at different rotation angles of upper rotation fingers. (b) Torques acting on upper rotation fingers at different rotation angles of lower rotation fingers. (c) The intersection of two curves represents rotation angles of lower and upper rotation fingers under $150 \mathrm{~V}$ driving voltage

From Fig. A.1 (a) the results of $\theta_{l}$ and $\theta_{u}$, at which the electrostatic torque acting on the lower rotation fingers is equal to the mechanical restoring torque from the lower springs, can be obtained graphically and are plotted as the solid curve in Fig. A.1 (c). In the same way, the results of $\theta_{l}$ and $\theta_{u}$, at which the electrostatic torque acting on the upper rotation fingers is equal to the mechanical restoring torque from the upper springs can be obtained graphically and plotted as the dash curve in Fig. A.1 (c). Then intersection of the two curves in Fig. A.1 (c) represents the rotation angles of the lower and upper rotation fingers at the driving voltage of $150 \mathrm{~V}$, which are $\theta_{l_{-} \max }$ and $\theta_{u_{-} \max }$. Since the above method does not consider the coupling effect between mechanical and electrical 
domains and is based on the simulation data of discrete rotation angles of $\theta_{l}$ and $\theta_{u}$, it is not suitable for simulating the performance of the actuator. But it is useful for approximately determining $\theta_{l_{-} \max }$ and $\theta_{u_{-} \max }$ and then determining the ranges of $h_{l}$ and $h_{u}$ through trial and error verifying iteration.

Using the above method, $\theta_{l_{-} \text {max } \_ \text {estimate }}$ and $\theta_{u_{-} \text {max } \_ \text {estimate }}$ are estimated to be $9.4^{\circ}$ and $3.9^{\circ}$. $\theta_{l_{-} m a x \_s s t i m a t e}$ and $\theta_{u_{-} \text {max_estimate }}$ are the estimated values of $\theta_{l_{-} \max }$ and $\theta_{u_{-} \max }$ using the above method. The trial and error verifying iteration has the following four steps.

Step 1: $\theta_{l \_m a x \_s s t i m a t e}$ and $\theta_{u_{-} m a x \_s t i m a t e}$ are rounded to the larger integers, i.e., $\theta_{l \_m a x \_s s t i m a t e}=10^{\circ}$ and $\theta_{u_{-} \text {max } \_ \text {estimate }}=4^{\circ}$ which are used to calculate the ranges of $h_{l}$ and $h_{u}$ according to Eqs. (2.5) to (2.8), i.e., $h_{l \_ \text {max_estimate }}$ and $h_{\text {u_max_estimate. }}$

Step 2: $h_{\_\_ \text {max_estimate }}$ and $h_{u_{-} \text {max_estimate }}$ are the estimated ranges of $h_{l}$ and $h_{u}$ are used for simulation to obtain $f_{\text {interdigitating_lower, }} f_{\text {interdigitating_upper }}$ and $f_{\text {non-interdigitating_upper }}$ and solve the maximum angles of lower and upper rotation fingers, i.e., $\theta_{l_{-} \text {max } \_ \text {model }}$ and $\theta_{u_{-} \text {max_model }}$ at the driving voltage of $150 \mathrm{~V}$ using the model in Chapter 2.

Step 3-1: If there exists solutions to $\theta_{l_{-} \text {max }}$ model and $\theta_{u_{-} \text {max }}$ model, , they are used to calculate maximum $h_{l}$ and $h_{u}$, i.e., $h_{l \_m a x \_m o d e l}$ and $h_{u \_m a x \_m o d e l}$. If $h_{l_{-} m a x \_m o d e l}$ and $h_{u \_m a x \_m o d e l}$ are smaller than the estimated ranges, i.e., $h h_{\_}$max_estimate and $h_{\text {u_max_estimate, }}$ then the estimated ranges are appropriate and the verification iteration ends. Otherwise the estimated ranges, i.e., $h_{l \_m a x \_s s t i m a t e}$ and $h_{u_{-} \text {max_estimate, are }}$ too small and Step 4 is the next step.

Step 3-2: If there is no solutions to $\theta_{l_{-} \text {max }}$ model and $\theta_{u_{-} \text {max } \_ \text {model }}$, the current estimated ranges, i.e., $h_{l \_m a x}$ estimate and $h_{u_{-} \text {max_estimate }}$ are too small and Step 4 is the next step.

Step 4: An increment of $10 \mu \mathrm{m}$ is added to the current $h_{l_{-} \text {max_estimate }}$ and $h_{u_{-} \text {max_estimate }}$ and then go to Step 2.

Using the above method, the ranges of $h_{l}$ and $h_{u}$ in the interdigitating and non-interdigitating area are determined, i.e., interdigitating area, $h_{l}: 0 \sim 50 \mu \mathrm{m}, h_{u}: 0 \sim 50 \mu \mathrm{m}$, non-interdigitating area, $h_{l}: 0 \sim 110 \mu \mathrm{m}, h_{u}: 0 \sim 50 \mu \mathrm{m}$. 


\section{Appendix B}

$$
\begin{gathered}
f_{\text {interdigitating_lower }}\left(h_{l}, h_{u}\right)=P_{00}+P_{10} h_{l}+P_{01} h_{u}+P_{20} h_{l}^{2}+P_{11} h_{l} h_{u}+P_{02} h_{u}^{2}+P_{30} h_{l}^{3}+P_{21} h_{l}^{2} h_{u}+P_{12} h h_{u}^{2} \\
+P_{03} h_{u}^{3}+P_{40} h_{l}^{4}+P_{31} h_{l}^{3} h_{u}+P_{22} h_{l}^{2} h_{u}^{2}+P_{13} h_{l} h_{u}^{3}+P_{50} h_{l}^{5}+P_{41} h_{l}^{4} h_{u}+P_{32} h_{l}^{3} h_{u}^{2}+P_{23} h_{l}^{2} h_{u}^{3}
\end{gathered}
$$

Where $P_{00}=2.751, P_{10}=7.195 \times 10^{-2}, P_{01}=2.438 \times 10^{-4}, P_{20}=-3.174 \times 10^{-2}, P_{11}=-2.493 \times 10^{-3}, P_{02}=$ -

$8.741 \times 10^{-3}, P_{30}=1.612 \times 10^{-3}, P_{21}=-5.648 \times 10^{-4}, P_{12}=7.295 \times 10^{-4}, P_{03}=9.391 \times 10^{-5}, P_{40}=-3.082 \times 10^{-}$ ${ }^{5}, P_{31}=6.562 \times 10^{-6}, P_{22}=2.633 \times 10^{-6}, P_{13}=-1.449 \times 10^{-5}, P_{50}=2.031 \times 10^{-7}, P_{41}=8.637 \times 10^{-8}, P_{32}=-$ $3.271 \times 10^{-7}, P_{23}=2.977 \times 10^{-7}$

$$
\begin{aligned}
f_{\text {interdigitating_upper }}( & \left.h_{l}, h_{u}\right)=P_{00}+P_{10} h_{l}+P_{01} h_{u}+P_{20} h_{l}^{2}+P_{11} h_{l} h_{u}+P_{02} h_{u}^{2}+P_{30} h_{l}^{3}+P_{21} h_{l}^{2} h_{u}+P_{12} h_{l} h_{u}^{2} \\
& +P_{03} h_{u}^{3}+P_{40} h_{l}^{4}+P_{31} h_{l}^{3} h_{u}+P_{22} h_{l}^{2} h_{u}^{2}+P_{13} h_{l} h_{u}^{3}+P_{04} h_{u}^{4}+P_{50} h_{l}^{5}+P_{41} h_{l}^{4} h_{u} \\
& +P_{32} h_{l}^{3} h_{u}^{2}+P_{23} h_{l}^{2} h_{u}^{3}+P_{14} h_{l} h_{u}^{4}
\end{aligned}
$$

Where $P_{00}=3.007, P_{10}=1.610 \times 10^{-1}, P_{01}=-7.337 \times 10^{-2}, P_{20}=-5.365 \times 10^{-4}, P_{11}=6.988 \times 10^{-4}, P_{02}=$ $1.102 \times 10^{-2}, P_{30}=-1.804 \times 10^{-4}, P_{21}=4.140 \times 10^{-4}, P_{12}=-5.683 \times 10^{-4}, P_{03}=4.885 \times 10^{-4}, P_{40}=3.259 \times$ $10^{-6}, P_{31}=-4.420 \times 10^{-6}, P_{22}=2.473 \times 10^{-6}, P_{13}=6.782 \times 10^{-6}, P_{04}=-5.415 \times 10^{-6}, P_{50}=-7.772 \times 10^{-9}$, $P_{41}=-9.964 \times 10^{-8}, P_{32}=2.976 \times 10^{-7}, P_{23}=-3.274 \times 10^{-7}, P_{14}=8.555 \times 10^{-8}$.

$$
\begin{gathered}
f_{\text {non-interdigitating_upper }}\left(h_{l}, h_{u}\right)=P_{00}+P_{10} h_{l}+P_{01} h_{u}+P_{11} h_{l} h_{u}+P_{02} h_{u}^{2}+P_{12} h_{l} h_{u}^{2} \\
+P_{03} h_{u}^{3}+P_{13} h_{l} h_{u}^{3}+P_{04} h_{u}^{4}+P_{14} h_{l} h_{u}^{4}+P_{05} h_{u}^{5}
\end{gathered}
$$

\begin{tabular}{|c|c|c|c|c|c|c|}
\hline \multirow{2}{*}{$\begin{array}{c}\text { Lens parameter } \\
(\text { Diameter }(\mathrm{mm}) \times \text { Focal } \\
\text { length }(\mathrm{mm}))\end{array}$} & \multirow{2}{*}{$\begin{array}{l}\text { Distance } \\
\text { from the } \\
\text { micromirror } \\
(\mathrm{mm})\end{array}$} & \multicolumn{4}{|c|}{$\begin{array}{l}\text { Geometric radius of the spots on the } \\
\text { image plane (deg) }\end{array}$} & \multirow{2}{*}{$\begin{array}{l}\text { Scanning distance on the } \\
\text { image plane }(\mathrm{mm})\end{array}$} \\
\hline & & $-1.5^{\circ}$ tilt & $0^{\circ}$ tilt & $1.5^{\circ}$ tilt & Average & \\
\hline $9 \times-9$ & 52.33 & 0.234 & 0.167 & 0.190 & 0.197 & 172 \\
\hline $9 \times-12$ & 50.66 & 0.146 & 0.12 & 0.115 & 0.127 & 124 \\
\hline $9 \times-18$ & 50.31 & 0.149 & 0.121 & 0.118 & 0.129 & 121 \\
\hline $12 \times-12$ & 49.74 & 0.146 & 0.119 & 0.115 & 0.126 & 124 \\
\hline $12 \times-24$ & 46.87 & 0.114 & 0.102 & 0.085 & 0.100 & 92 \\
\hline $12 \times-18$ & 50.87 & 0.157 & 0.138 & 10117 & 0.137 & 128 \\
\hline
\end{tabular}

Where $P_{00}=-7.826 \times 10^{-2}, P_{10}=3.120 \times 10^{-2}, P_{01}=-5.305 \times 10^{-1}, P_{11}=-2.423 \times 10^{-3}, P_{02}=8.097 \times 10^{-1}$, $P_{12}=5.462 \times 10^{-4}, P_{03}=-5.021 \times 10^{-1}, P_{13}=1.571 \times 10^{-3}, P_{04}=-1.163 \times 10^{-1}, P_{14}=-8.357 \times 10^{-4}, P_{05}=$ $1.314 \times 10^{-1}$.

\section{Appendix C Optical optimization results}

Table C.1 Optimization results of various lenses. 


\section{Appendix D Bonding process of the protective glass}

The micromirror after the fabrication is positioned in a CQFP chip without cover. Due to the small size of the micromirror, the particles and moistures in the surrounding air and the humidity level of the air may cause the failure of the micromirror. As a result, it is necessary to isolate the micromirror with the outside environment without blocking the light path.

A $10 \mathrm{~mm} \times 10 \mathrm{~mm}$ square AR window with high light transmission is selected to bond with the chip. Epoxy adhesive due to its superior abilities of high strength, resistance to high temperature, humidity and chemical is suitable for this purpose. The Loctite E-30CL epoxy is selected as the boding glue which is stable in the temperature range $-65^{\circ} \sim 250^{\circ}$ and is widely used to bond glass and ceramic. It begins to harden in $30 \mathrm{~min}$ and reaches full strength in 24 hours.

In order to avoid damaging the device while bonding, a reliable bonding process has been developed to bond the window on top of the micromirror.

The setup for the bonding is shown in Fig. D.1 There is a vertical slider sliding along a tall cylinder post. A "L" shape fixture is attached with the vertical slider. A cavity is made on the surface of a Teflon cuboid. The size of the cavity is a little larger than the AR glass so that it can fit in.

The process is listed below:

(1) Peel off the plastic protection layer on both side of the AR glass, and leave positioned on a electrical grounded metal board for about 5 mins and then put it in the cavity on the Teflon for later use. The AR glass comes with a thin plastic protection layers at each side of it. It has been proved that when peeling off the protection layer, the electrostatic charges will be generated on the glass. For some tests, if it is positioned on top of the micromirror with large amount of charge it damaged the micromirror due to the possible large electrostatic force between the glass and the microactuator. Teflon material due to its non-stick nature is used to hold the glass during the bonding process so that the glass or the micromirror can be easily release from it after the bonding is finished.

(2) Squeeze the epoxy resin and hardener onto a flat and clean solid surface such as a piece of silicon wafer and mix them until the color is uniform. Epoxy adhesive comes with resin and hardener which need to be mixed to start the curing process. 
(3) Take off the metal lid on the chip and use double side tape to fix the chip on the "L" shape fixture with the chip facing down, as shown in the Fig. D.1 (a) above. In this process, the micromirror will be exposed directly to the operation environment and the potential to be damaged increases with the time. As a result, it is necessary for the operator to maintain a small movement in order to avoid too much air flow around the micromirror.

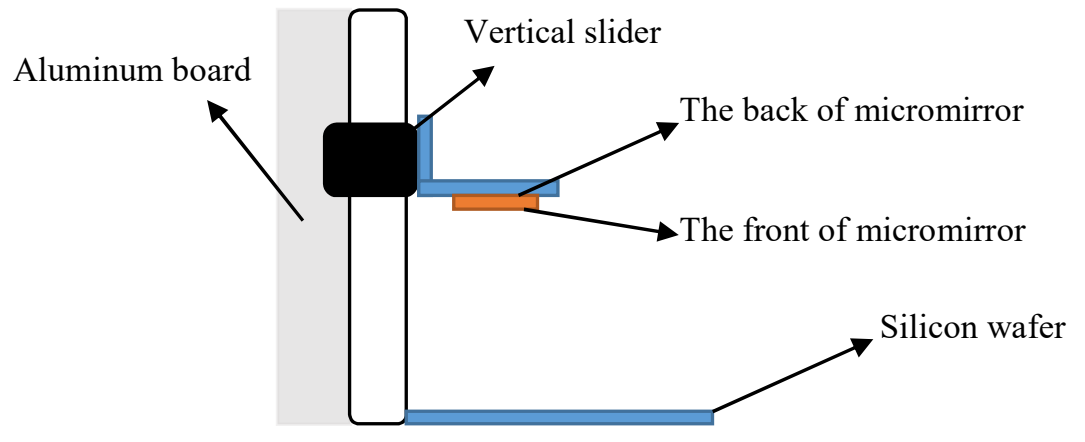

(a)

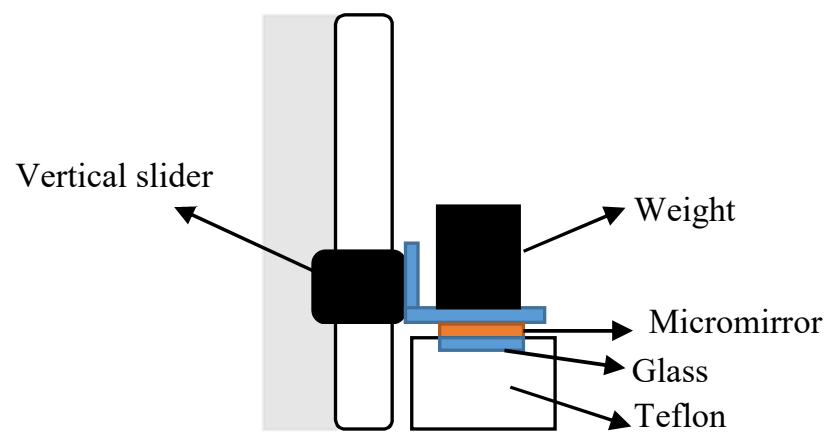

(b)

Fig. D.1 Setup for the bonding process.

(4) Use a piece of thin and clean board such as a piece of wafer to press and slide over the mixed glue that was prepared until a uniform thin layer of the mixture is formed. This step may be repeated serval time to guarantee the generation of the thin and uniform layer of glue. It is important to generate a thin mixture layer so that when the micromirror is contacting with the mixture, it won't have too much mixture squeezed into the cavity of the CQFP chip and cause damage to the micromirror.

(5) Put the wafer with a thin layer of epoxy right under the chip. Lower the "L" shape steadily and slowly until the front face of the chip makes contact with the mixture layer. 
(6) Then slowly lift the "L" shape and take off the chip. Use hand to position the chip into the cavity which contains the glass. Make sure that the front surface of the chip is flat and has a full contact with the glass.

(7) Lower the "L" shape to apply bonding force to the back of the chip. Extra weight can be put on the "L" shape to enhance the bonding force. The device will be ready to use after 24 hours.

The real bonding structure and the bonded micromirror is shown in Fig. D.2. The whole setup is on an optical bench. The black slider with a "L" shape attached to it is able to slide vertically along the pillar. An aluminum board is inserted into the opening at the back of the black slider to prevent it from rotating around the cylinder. In Fig. D.2 the "L" shape is applying the bonding force to the back of a micromirror chip. The front of the chip is attached to an AR glass with epoxy adhesive sandwiched between them. A clean silicon wafer (with a thick glass substrate) is used to hold the epoxy. A micromirror chip without bonding, a piece of AR glass with protection plastic cover and a Teflon brick is shown in Fig. D.3 (a). More than 10 chips has been bonded using the same process and it has been proved that the bonding process is very reliable and can be accomplished under the normal lab environment. A bonded micromirror is shown in Fig. D.3 (b).

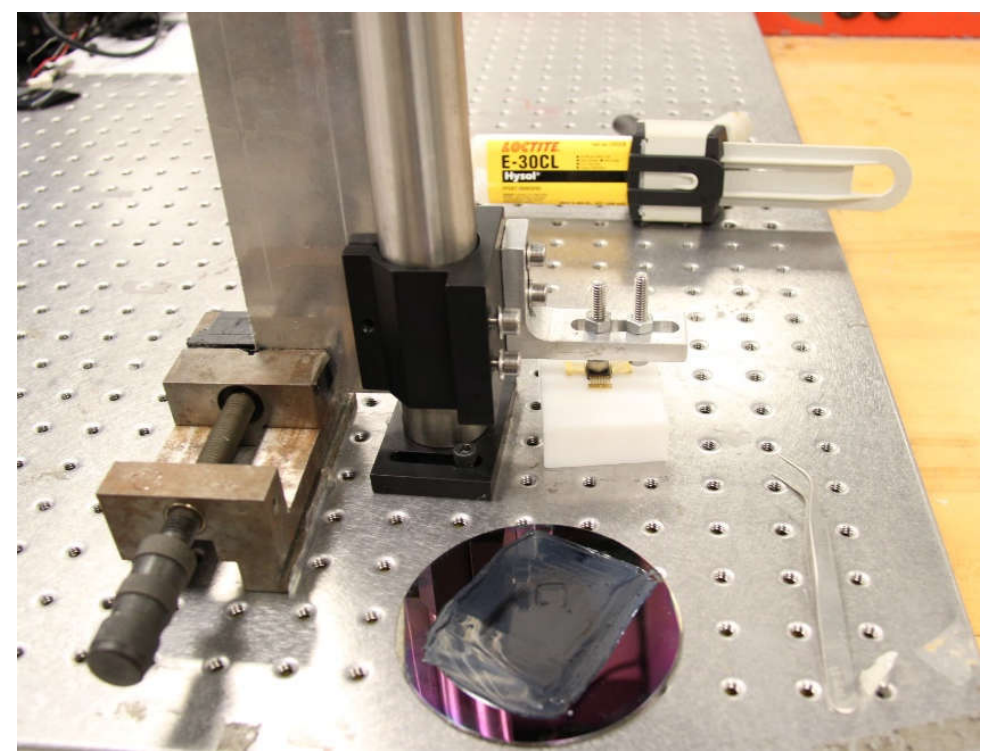

Fig. D.2 The bonding setup of the micromirror. 


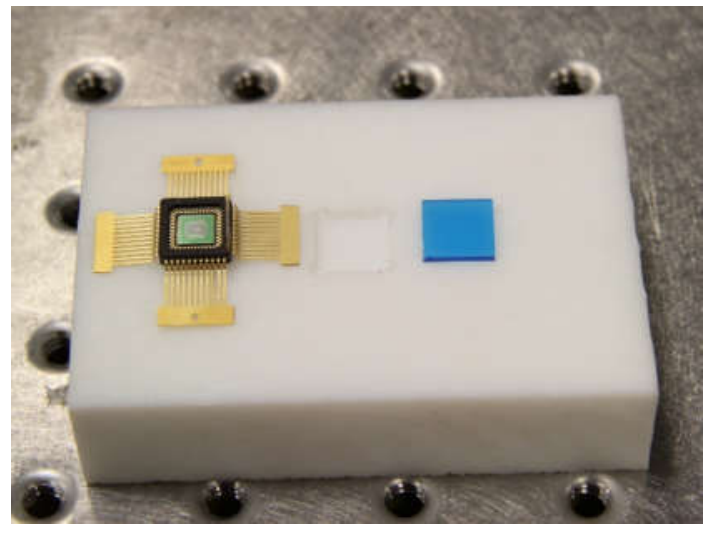

(a)

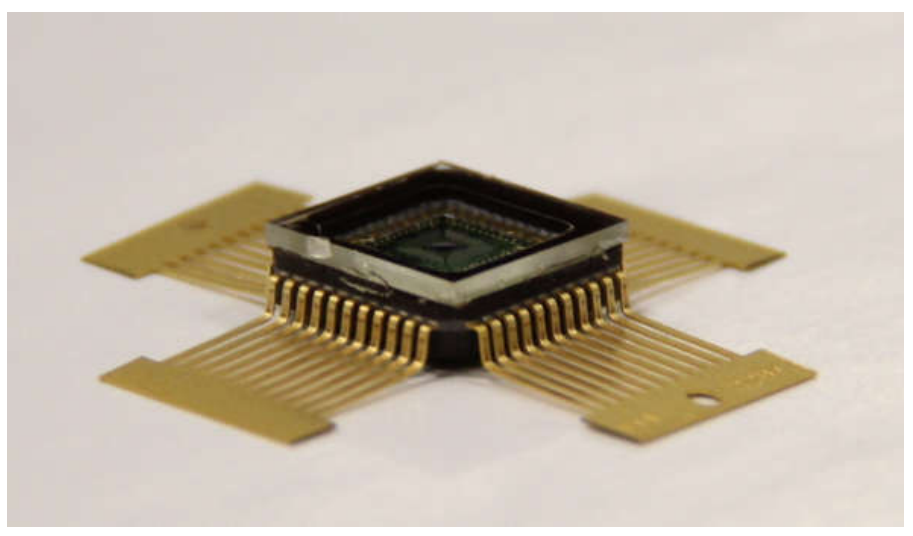

(b)

Fig. D.3 (a) The micromirror chip (before bonding) and an AR glass (with protection covers) on a Tefolon (b) The micromirror chip after bonding 


\section{Reference List}

[1] C. Liu, Foundations of MEMS, 2nd ed. Prentice Hall, 2011.

[2] V. Kaajakari, Practical MEMS: design of microsystems, accelerometers, gyroscopes, RF MEMS, optical MEMS, and microfluidic systems. Small Gear Publishing, 2009.

[3] L. Wu and H. Xie, "A large vertical displacement electrothermal bimorph microactuator with very small lateral shift," Sensors Actuators A Phys., vol. 145-146, pp. 371-379, 2008.

[4] A. Michael, C. Y. Kwok, S. Member, K. Yu, and M. R. Mackenzie, "A novel bistable twoway actuated out-of-plane electrothermal microbridge," J. Microelectromechanical Syst., vol. 17 , no. 1, pp. 58-69, 2008.

[5] Y. Xu, J. Singh, T. Selvaratnam, and N. Chen, "Two-axis gimbal-less electrothermal micromirror for large-angle circumferential scanning," IEEE J. Sel. Top. Quantum Electron., vol. 15, no. 5, pp. 1432-1438, 2009.

[6] S. Pal and H. Xie, "A curved multimorph based electrothermal micromirror with large scan range and low drive voltage," Sensors Actuators A Phys., vol. 170, no. 1-2, pp. 156-163, 2011.

[7] S. R. Samuelson, H. Xie, and S. Member, "A large piston displacement MEMS mirror With electrothermal ladder actuator arrays for ultra-low tilt applications," $J$. Microelectromechanical Syst., vol. 23, no. 1, pp. 39-49, 2014.

[8] J. J. Bernstein, W. P. Taylor, J. D. Brazzle, et al., "Electromagnetically actuated mirror arrays for use in 3-D optical switching applications," J. Microelectromechanical Syst., vol. 13, no. 3, pp. 526-535, 2004.

[9] A. D. Yalcinkaya, H. Urey, D. Brown, T. Montague, and R. Sprague, "Two-axis electromagnetic microscanner for high resolution displays," J. Microelectromechanical Syst., vol. 15, no. 4, pp. 786-794, 2006.

[10] V. F.-G. Tseng, J. Li, X. Zhang, et al., "An electromagnetically actuated micromirror with precise angle control for harsh environment optical switching applications," Sensors Actuators A Phys., vol. 206, pp. 1-9, 2014.

[11] A. R. Cho, A. Han, S. Ju, et al., "Electromagnetic biaxial microscanner with mechanical 
amplification at resonance," Opt. Express, vol. 23, no. 13, pp. 16792-16802, 2015.

[12] X. Lv, W. Wei, X. Mao, et al., "A novel MEMS electromagnetic actuator with large displacement," Sensors Actuators A Phys., vol. 221, pp. 22-28, 2015.

[13] F. Filhol, E. Defaÿ, C. Divoux, C. Zinck, and M.-T. Delaye, "Resonant micro-mirror excited by a thin-film piezoelectric actuator for fast optical beam scanning," Sensors Actuators A Phys., vol. 123-124, pp. 483-489, 2005.

[14] K. H. Koh, T. Kobayashi, F. L. Hsiao, and C. Lee, "Characterization of piezoelectric PZT beam actuators for driving 2D scanning micromirrors," Sensors Actuators, A Phys., vol. 162, no. 2 , pp. 336-347, 2010.

[15] S. C. Shen, Y. J. Wang, and M. T. Shih, "A novel dual-screen projection system using a balance-type micromirror with a piezoelectric actuator," Sensors Actuators A Phys., vol. 199, pp. 80-88, 2013.

[16] T. Naono, T. Fujii, M. Esashi, and S. Tanaka, "A large-scan-angle piezoelectric MEMS optical scanner actuated by a Nb-doped PZT thin film," J. Micromechanics Microengineering, vol. 24, no. 1, p. 015010, 2014.

[17] C.-D. Chen, Y.-H. Lee, and C.-S. Yeh, "Design and vibration analysis of a piezoelectricactuated MEMS scanning mirror and its application to laser projection," Smart Mater. Struct., vol. 23, no. 12, p. 125007, 2014.

[18] Y. Lin, J. Chiou, W. Lin, Y. Lin, and S. Wu, "The design and assembly of surfacemicromachined optical switch for optical add/drop multiplexer application," IEEE Trans. Adv. Packag., vol. 26, no. 3, pp. 261-267, 2003.

[19] V. A. Aksyuk, F. Pardo, D. Carr, et al., "Beam-steering micromirrors for large optical crossconnects," J. Light. Technol., vol. 21, no. 3, pp. 634-642, 2003.

[20] C.-H. Ji, M. Choi, S.-C. Kim, et al., "An electrostatic scanning micromirror with diaphragm mirror plate and diamond-shaped reinforcement frame," $J$. Micromechanics Microengineering, vol. 16, no. 5, pp. 1033-1039, 2006.

[21] X. M. Zhang, A. Q. Liu, C. Lu, F. Wang, and Z. S. Liu, "Polysilicon micromachined fiberoptical attenuator for DWDM applications," Sensors Actuators A Phys., vol. 108, no. 1-3, 
pp. 28-35, 2003.

[22] L. Li, J. Zawadzka, and D. Uttamchandani, "Integrated self-assembling and holding technique applied to a 3-D MEMS variable optical attenuator," J. Microelectromechanical Syst., vol. 13, no. 1, pp. 83-90, 2004.

[23] Y. Liu, J. Xu, S. Zhong, and Y. Wu, "Large size MEMS scanning mirror with vertical comb drive for tunable optical filter," Opt. Lasers Eng., vol. 51, no. 1, pp. 54-60, 2013.

[24] P. F. Van Kessel, L. J. Hornbeck, R. E. Meier, and M. R. Douglass, "A MEMS-based projection display," Proc. IEEE, vol. 86, no. 8, pp. 1687-1704, 1998.

[25] V. Milanovic, G. A. Matus, and D. T. McCormick, "Gimbal-less monolithic silicon actuators for tip-tilt-piston micromirror applications," IEEE J. Sel. Top. Quantum Electron., vol. 10, no. 3, pp. 462-471, 2004.

[26] U. Hofmann, J. Janes, and H.-J. Quenzer, "High-Q MEMS resonators for laser beam scanning displays," Micromachines, vol. 3, no. 4, pp. 509-528, 2012.

[27] S. T. S. Holmström, U. Baran, H. Urey, and S. Member, "MEMS laser scanners : a review," J. Microelectromechanical Syst., vol. 23, no. 2, pp. 259-275, 2014.

[28] O. Degani, E. Socher, A. Lipson, et al., "Pull-in Study of an electrostatic torsion microactuator," J. Microelectromechanical Syst., vol. 7, no. 4, pp. 373-379, 1998.

[29] Y. Nemirovsky, O. Bochobza-degani, and S. Member, "A methodology and model for the pull-in parameters of electrostatic actuators," J. Microelectromechanical Syst., vol. 10, no. 4, pp. 601-615, 2001.

[30] W.-M. Zhang, H. Yan, Z.-K. Peng, and G. Meng, "Electrostatic pull-in instability in MEMS/NEMS: a review," Sensors Actuators A Phys., vol. 214, pp. 187-218, 2014.

[31] H. Ren, F. Tao, W. Wang, and J. Yao, "An out-of-plane electrostatic actuator based on the lever principle," J. Micromechanics Microengineering, vol. 21, no. 4, p. 045019, 2011.

[32] C.-H. Han, D.-H. Choi, and J.-B. Yoon, "Parallel-plate MEMS variable capacitor with superior linearity and large tuning ratio using a levering structure," $J$. Microelectromechanical Syst., vol. 20, no. 6, pp. 1345-1354, 2011.

[33] Y.-H. Song, C.-H. Han, M.-W. Kim, J. O. Lee, and J.-B. Yoon, "An electrostatically 
actuated stacked-electrode MEMS relay with a levering and torsional spring for power applications," J. Microelectromechanical Syst., vol. 21, no. 5, pp. 1209-1217, 2012.

[34] C. Han, D. Choi, H. Yang, Y. Yoon, and J. Yoon, "Voltage-controlled C - V response tuning in a parallel plate MEMS variable capacitor," J. Microelectromechanical Syst., vol. 22, no. 6, pp. 1403-1413, 2013.

[35] M. a Rosa, D. De Bruyker, A. R. Völkel, E. Peeters, and J. Dunec, “A novel external electrode configuration for the electrostatic actuation of MEMS based devices," $J$. Micromechanics Microengineering, vol. 14, no. 4, pp. 446-451, 2004.

[36] J. Su, H. Yang, P. Fay, W. Porod, and G. H. Bernstein, “A surface micromachined offsetdrive method to extend the electrostatic travel range," J. Micromechanics Microengineering, vol. 20, no. 1, pp. 125-35, 2009.

[37] J. Small, A. Fruehling, A. Garg, X. Liu, and D. Peroulis, "DC-dynamic biasing for $>50 \times$ switching time improvement in severely underdamped fringing-field electrostatic MEMS actuators," J. Micromechanics Microengineering, vol. 22, no. 12, p. 125029, 2012.

[38] J. Small, W. Irshad, A. Fruehling, et al., "Electrostatic fringing-field actuation for pull-in free RF-MEMS analogue tunable resonators," J. Micromechanics Microengineering, vol. 22, no. 9, p. 095004, 2012.

[39] E. Gallagher, W. Moussa, and M. McDermott, "A review of fabrication processes for vertical comb drives," Microsyst. Technol., vol. 18, no. 4, pp. 381-397, 2012.

[40] J. I. Seeger and S. B. Crary, "Stabilization of electrostatically actuated mechanical devices," in IEEE International conference on solid-state sensors and actuators, 1997, pp. 11331136.

[41] D. Elata and a. Hirshberg, "A novel method for measuring the strength of microbeams," $J$. Microelectromechanical Syst., vol. 15, no. 2, pp. 396-405, 2006.

[42] B. Rivlin and D. Elata, "Design of nonlinear springs for attaining a linear response in gapclosing electrostatic actuators,” Int. J. Solids Struct., vol. 49, no. 26, pp. 3816-3822, 2012.

[43] S. Shmulevich, B. Rivlin, I. Hotzen, and D. Elata, "A Gap-Closing Electrostatic Actuator With a Linear Extended Range,” J. Microelectromechanical Syst., vol. 22, no. 5, pp. 1109- 
$1114,2013$.

[44] J. I. Seeger and B. E. Boser, "Charge control of parallel-plate, electrostatic actuators and the tip-in instability," J. Microelectromechanical Syst., vol. 12, no. 5, pp. 656-671, 2003.

[45] J. I. Seeger and B. E. Boser, "Dynamics and control of parallel-plate actuators beyond the electrostatic instability," Transducers, pp. 474-477, 1999.

[46] E. K. Chan and R. W. Dutton, "Electrostatic micromechanical actuator with extended range of travel," J. Microelectromechanical Syst., vol. 9, no. 3, pp. 321-328, 2000.

[47] R. Nadal-Guardia, a. Dehé, R. Aigner, and L. M. Castañer, "Current drive methods to extend the range of travel of electrostatic microactuators beyond the voltage pull-in point," J. Microelectromechanical Syst., vol. 11, no. 3, pp. 255-263, 2002.

[48] D. H. S. Maithripala, J. M. Berg, and W. P. Dayawansa, "Control of an electrostatic microelectromechanical system using static and dynamic output feedback," J. Dyn. Syst. Meas. Control. Asme, vol. 127, no. September 2005, pp. 443-450, 2005.

[49] M. Vagia, G. Nikolakopoulos, and A. Tzes, "Design of a robust PID-control switching scheme for an electrostatic micro-actuator," Control Eng. Pract., vol. 16, no. 11, pp. 13211328, 2008.

[50] D. Hah, S. Huang, H. Nguyen, et al., "Low voltage MEMS analog micromirror arrays with hidden vertical comb-drive actuators," pp. 11-14, 2002.

[51] J. Wang, "Silicon-on-insulator out-of-plane electrostatic actuator with in situ capacitive position sensing," J. Micro/Nanolithography, MEMS, MOEMS, vol. 11, no. 3, p. 033006, 2012.

[52] U. Krishnamoorthy and O. Solgaard, "Self-aligned vertical electrostatic combdrives for micromirror actuation," J. Microelectromechanical Syst., vol. 12, no. 4, pp. 458-464, 2003.

[53] D. Hah, C.-A. Choi, C.-K. Kim, and C.-H. Jun, "A self-aligned vertical comb-drive actuator on an SOI wafer for a 2D scanning micromirror," J. Micromechanics Microengineering, vol. 14, no. 8, pp. 1148-1156, 2004.

[54] X.-Y. Li, Q. Jin, D.-Y. Qiao, et al., "Design and fabrication of a resonant scanning micromirror suspended by $\mathrm{V}$ shaped beams with vertical electrostatic comb drives," 
Microsyst. Technol., vol. 18, no. 3, pp. 295-302, 2011.

[55] T. Sasaki and K. Hane, "Varifocal micromirror integrated with comb-drive scanner on silicon-on-insulator wafer," J. Microelectromechanical Syst., vol. 21, no. 4, pp. 971-980, 2012.

[56] R. Bauer, L. Li, D. Uttamchandani, and S. Member, "Dynamic properties of angular vertical comb-drive scanning micromirrors with electrothermally cotrolled variable offset," $J$. Microelectromechanical Syst., vol. 23, no. 4, pp. 1-10, 2014.

[57] D. Hah, P. R. Patterson, H. D. Nguyen, H. Toshiyoshi, and M. C. Wu, "Theory and experiments of angular vertical comb-drive actuators for scanning micromirrors," IEEE $J$. Sel. Top. Quantum Electron., vol. 10, no. 3, pp. 505-513, 2004.

[58] D. Jung, T. Sandner, D. Kallweit, and H. Schenk, "Vertical comb drive microscanners for beam steering, linear scanning, and laser projection applications," in Proc. of SPIE, 2012, vol. 8252 , p. $82520 \mathrm{U}$.

[59] T. Sandner, C. Drabe, H. Schenk, A. Kenda, and W. Scherf, "Translatory MEMS actuators for optical path length modulation in miniaturized Fourier-transform infrared spectrometers," J. Micro/Nanolithography, MEMS, MOEMS, vol. 7, no. 2, p. 021006, 2008.

[60] J. Chiou, C. Kou, and Y. Lin, "A micromirror with large static rotation and vertical actuation," IEEE J. Sel. Top. Quantum Electron., vol. 13, no. 2, pp. 297-303, 2007.

[61] Y. Eun, H. Na, J. Choi, J. Lee, and J. Kim, "Angular vertical comb actuators assembled onchip using in-plane electrothermal actuators and latching mechanisms," Sensors Actuators A Phys., vol. 165, no. 1, pp. 94-100, 2011.

[62] U. Krishnamoorthy, D. Lee, and O. Solgaard, "Chapter 9. Vertical combdrive actuator: design and fabrication for micromirror applications," in MEMS/NEMS Handbook Techniques and Applications Volume 5 Medical Applications and MOEMS, 2006, pp. 393421.

[63] Z. Hailu, S. He, and R. Ben Mrad, "A novel vertical comb-drive electrostatic actuator using a one layer process," J. Micromechanics Microengineering, vol. 24, no. 11, p. 115016, 2014.

[64] Z. Hailu, S. He, and R. Ben Mrad, "Hybrid micro electrostatic actuator," Microsyst. 
Technol., pp. 1-9, 2015.

[65] M. Sasaki, D. Briand, W. Noell, N. F. De Rooij, and K. Hane, "Three-dimensional SOIMEMS constructed by buckled bridges and vertical comb drive actuator," IEEE J. Sel. Top. Quantum Electron., vol. 10, no. 3, pp. 455-461, 2004.

[66] J. Kim, H. Choo, L. Lin, and R. S. Muller, "Microfabricated torsional actuators using selfaligned plastic deformation of silicon," J. Microelectromechanical Syst., vol. 15, no. 3, pp. 553-562, 2006.

[67] J. Kim, D. Christensen, and L. Lin, "Micro vertical comb actuators by selective stiction process," Sensors Actuators A Phys., vol. 127, no. 2, pp. 248-254, 2006.

[68] J. Kim, S. Park, and D.-I. Cho, "A novel electrostatic vertical actuator fabricated in one homogeneous silicon wafer using extended SBM technology," Sensors Actuators A Phys., vol. 97-98, pp. 653-658, 2002.

[69] Q. X. Zhang, A. Q. Liu, J. Li, and A. B. Yu, "Fabrication technique for microelectromechanical systems vertical comb-drive actuators on a monolithic silicon substrate," J. Vac. Sci. Technol. B, vol. 23, pp. 32-41, 2005.

[70] J. M.-L. Tsai, H.-Y. Chu, J. Hsieh, and W. Fang, "The BELST II process for a silicon highaspect-ratio micromaching vertical comb actuator and its applications," J. Micromechanics Microengineering, vol. 14, no. 2, pp. 235-241, 2004.

[71] C. Chan, C. Hsu, M. Wu, et al., "A novel differential capacitive-sensing dual-axis accelerometer design using pendulum-proofmass, gimbal-springs, and harm verticalcombs," Solid-State Sensors, Actuators Microsystems Conf., pp. 1944-1947, 2009.

[72] M. Wu and W. Fang, "Design and fabrication of MEMS devices using the integration of MUMPs, trench-refilled molding, DRIE and bulk silicon etching processes," $J$. Micromechanics Microengineering, vol. 15, no. 3, pp. 535-542, 2005.

[73] M. Wu and W. Fang, "A molded surface-micromachining and bulk etching release (MOSBE) fabrication platform on (1 111$) \mathrm{Si}$ for MOEMS," J. Micromechanics Microengineering, vol. 16, no. 2, pp. 260-265, 2006.

[74] J. L. a Yeh, H. Jiang, and N. C. Tien, "Integrated polysilicon and DRIE bulk silicon 
micromachining for an electrostatic torsional actuator," J. Microelectromechanical Syst., vol. 8, no. 4, pp. 456-465, 1999.

[75] E. Carr, S. Olivier, and O. Solgaard, "Large-stroke self-aligned vertical comb drive actuators for adaptive optics applications," in MEMS/MOEMS Components and Their Applications III, 2006, vol. 6113, p. 61130T.

[76] D. Lee, U. Krishnamoorthy, K. Yu, and O. Solgaard, "Single-crystalline silicon micromirrors actuated by self-aligned vertical electrostatic combdrives with piston-motion and rotation capability," Sensors Actuators A Phys., vol. 114, no. 2-3, pp. 423-428, 2004.

[77] K. Kumar, K. Hoshino, and X. Zhang, "Handheld subcellular-resolution single-fiber confocal microscope using high-reflectivity two-axis vertical combdrive silicon microscanner," Biomed. Microdevices, vol. 10, no. 5, pp. 653-660, 2008.

[78] K. Kumar, "CMOS-compatible 2-axis sefl-aligned vertical comb-drive micromirror for large field-of-view microendoscopes," in IEEE 22nd International Conference on Micro Electro Mechanical Systems, 2009, pp. 1015-1018.

[79] D. H. D. Hah, C.-A. C. C.-A. Choi, C.-H. J. C.-H. Jun, and Y. T. K. Y. T. Kim, “A selfaligned vertical comb-drive actuator using surface micromachining for scanning micromirrors," in 2003 IEEE/LEOS International Conference on Optical MEMS, 2003, pp. 151-152.

[80] W. T. Lin, J. C. Chiou, and C. Tsou, "A self-aligned fabrication method of dual comb drive using multilayers SOI process for optical MEMS applications,” vol. 11, pp. 204-209, 2005.

[81] C. Tsou, W. T. Lin, C. C. Fan, and B. C. S. Chou, "A novel self-aligned vertical electrostatic combdrives actuator for scanning micromirrors," J. Micromechanics Microengineering, vol. 15, no. 4, pp. 855-860, 2005.

[82] E. T. Carlen, K. H. Heng, S. Bakshi, A. Pareek, and C. H. Mastrangelo, "High-aspect ratio vertical comb-drive actuator with small self-aligned finger gaps," J. Microelectromechanical Syst., vol. 14, no. 5, pp. 1144-1155, 2005.

[83] N. Kouma, O. Tsuboi, H. Soneda, S. Ueda, and I. Sawaki, "Fishbone-shaped qrtical comb actuator for dual-axis 1-D analog micromirror array," in The 13th International Conference on Solid-State Sensors, Actuators and Microsystems, 2005, pp. 980 - 983. 
[84] J.-W. Cho, Y.-H. Park, Y.-C. Ko, et al., "Electrostatic 1D microscanner with vertical combs for HD resolution display," in Proc. of SPIE, 2007, vol. 6466, p. 64660B.

[85] M. Kim, J.-H. Park, J.-A. Jeon, et al., "High fill-factor micromirror array using a selfaligned vertical comb drive actuator with two rotational axes," J. Micromechanics Microengineering, vol. 19, no. 3, p. 035014, 2009.

[86] T. Tsuchiya and H. Funabashi, "A z-axis differential capacitive SOI accelerometer with vertical comb electrodes," Sensors Actuators A Phys., vol. 116, no. 3, pp. 378-383, 2004.

[87] C. Wang, G. Yan, and G. Wu, "A bulk micromachined lateral axis gyroscope with vertical sensing comb capacitors," in The 13th International Conference on Solid-State Sensors, Actuators and Microsystems, 2005, pp. 121-124.

[88] X. Liu, Z. Yang, G. Yan, et al., "Design and Fabrication of a lateral axis Gyroscope with Asymmetric Comb-Fingers as Sensing Capacitors," in 1st IEEE International Conference on Nano/Micro Engineered and Molecular Systems, 2006, pp. 762-765.

[89] H. Hamaguchi, K. Sugano, T. Tsuchiya, and O. Tabata, "A differential capacitive three-axis SOI accelerometer using vertical comb electrodes," in The 14th International Conference on Solid-State Sensors, Actuators and Microsystems, 2007, pp. 1483-1486.

[90] M. Zickar, M. Mita, M. Ataka, and H. Fujita, "Low cross talk design and simple fabrication process of electrostatic vertical comb-drive actuators for positioning application," IEEJ Trans. Electr. Electron. Eng., vol. 2, no. 3, pp. 289-294, 2007.

[91] J. Chung and W. Hsu, "Fabrication of a polymer-based torsional vertical comb drive using a double-side partial exposure method," J. Micromechanics Microengineering, vol. 18, no. 3, p. 035014, 2008.

[92] J.-H. Lee, Y.-C. Ko, B.-S. Choi, J.-M. Kim, and D. Y. Jeon, "Bonding of silicon scanning mirror having vertical comb fingers," J. Micromechanics Microengineering, vol. 12, no. 5, p. $320,2002$.

[93] Y.-C. Ko, J.-W. Cho, Y.-K. Mun, et al., "Eye-type scanning mirror with dual vertical combs for laser display," Sensors Actuators A Phys., vol. 126, no. 1, pp. 218-226, 2006.

[94] E. Iwase, H. Onoe, K. Matsumoto, and I. Shimoyama, "Hidden vertical comb-drive actuator 
on PDMS fabricated by parts-transfer," Proc. IEEE Int. Conf. Micro Electro Mech. Syst., pp. 116-119, 2008.

[95] E. Gallagher and W. Moussa, "A study of the effect of the fringe fields on the electrostatic force in vertical Comb drives," Sensors, vol. 14, no. 11, pp. 20149-20164, 2014.

[96] U. Krishnamoorthy, K. Li, K. Yu, et al., "Dual-mode micromirrors for optical phased array applications," Sensors Actuators, A Phys., vol. 97-98, pp. 21-26, 2002.

[97] S. He and R. Ben Mrad, "Design , modeling, and demonstration of a MEMS repulsiveforce out-of-plane electrostatic micro actuator," J. Microelectromechanical Syst., vol. 17, no. 3, pp. 532-547, 2008.

[98] D. Elata, "Modeling the electromechanical response of electrostatic actuators," in Mems/Nems Handbook:Sensors and Actuators, 2006, pp. 93-119.

[99] I. Rezadad, J. Boroumand, E. M. Smith, and R. E. Peale, "Micro electro mechanical cantilever with electrostatically controlled tip contact," Appl. Phys. Lett., vol. 105, p. 033514, 2014.

[100] I. Rezadad, J. Boroumand Azad, E. M. Smith, A. Alhasan, and R. E. Peale, "Vertical electrostatic force in MEMS cantilever IR sensor," in Infrared Technology and Applications XL, 2014, vol. 9070, p. 90701U.

[101] S. He and R. Ben Mrad, "Development of a novel translation micromirror for adaptive optics," in Proc. of SPIE, 2003, vol. 5264, pp. 154-161.

[102] S. He and R. Ben Mrad, "Large-stroke microelectrostatic actuators for vertical translation of micromirrors used in adaptive optics," IEEE Trans. Ind. Electron., vol. 52, no. 4, pp. 974-983, 2005.

[103] W. Wang, F. Tao, Q. Wang, et al., "A 19-element segmented MEMS deformable mirror based on electrostatic repulsive-force actuator," in Proc. of SPIE, 2013, vol. 8617, p. 861702 .

[104] S. He and R. Ben Mrad, "Repulsive force actuated rotary micromirror," in Optomechatronic Sensors, Actuators, and Control, 2004, vol. 5602, pp. 12-18.

[105] S. He, R. Ben Mrad, and J. S. Chang, "Development of a high-performance 
microelectrostatic repulsive-force rotation actuator," J. Microelectromechanical Syst., vol. 19, no. 3, pp. 561-569, 2010.

[106] Y. Xue and S. He, "Novel automated microassembly mechanism based on on-chip actuators," J. Micro/Nanolithography, MEMS, MOEMS, vol. 12, no. 1, p. 013003, 2013.

[107] S. He, R. Ben Mrad, and J. Chong, "Repulsive-force out-of-plane large stroke translation micro electrostatic actuator," J. Micromechanics Microengineering, vol. 21, no. 7, p. $075002,2011$.

[108] J. Chong, S. He, and R. Ben Mrad, "Development of a vector display system based on a surface-micromachined micromirror," IEEE Trans. Ind. Electron., vol. 59, no. 12, pp. 4863-4870, 2012.

[109] C. Fan, S. He, J. Chong, R. Ben Mrad, and L. Feng, "Development of a micromirror based laser vector scanning automotive HUD," in IEEE International Conference on Mechatronics and Automation, 2011, pp. 75-79.

[110] J. M. Bustillo, R. T. Howe, and R. S. Muller, "Surface micromachining for microelectromechanical systems," in Proceedings of the IEEE, 1998, vol. 86, no. 8, pp. $1552-1574$.

[111] G. D. J. Su, H. Toshiyoshi, and M. C. Wu, "Surface-micromachined 2-D optical scanners with high-performance single-crystalline silicon micromirrors," IEEE Photonics Technol. Lett., vol. 13, no. 6, pp. 606-608, 2001.

[112] A. Cowen, B. Hardy, R. Mahadevan, and S. Wilcenski, PolyMUMPs design handbook revision 13.0. MEMSCAP Inc, 2011.

[113] “Small Beam Diameter Scanning Galvo Mirror Systems," THORLABS, Inc. [Online]. Available: https://www.thorlabs.com/newgrouppage9.cfm?objectgroup_id=3770.

[114] "Two axis galvanometer scanning system," ZAMISEL. [Online]. Available: http://www.zamisel.com/SSpostavka2.html.

[115] “Laser projection assembly guide," Assembly Guidance Systems, Inc. [Online]. Available: http://www.assemblyguide.com/laserguidesys.html.

[116] "Laser show," Canton Laserphoto Electronic Equipment Co.Ltd. [Online]. Available: 
http://www.laserphoto.org/en/laser.asp.

[117] V. Milanovi, K. Castelino, and D. T. Mccormick, "Highly adaptable MEMS-based display with wide projection angle," in IEEE 20th International Conference on Micro Electro Mechanical Systems, 2007, pp. 143-146.

[118] E. Buckley, "Eye safety analysis of current laser based scanned beam projection systems," J. Soc. Inf. Disp., vol. 18, no. 11, p. 944, 2010.

[119] E. Buckley, "Detailed eye-safety analysis of laser-based scanned-beam projection systems," J. Disp. Technol., vol. 8, no. 3, pp. 166-173, 2012.

[120] M. Freeman, M. Champion, and S. Madhavaan, "Scanned laser pico-projected: seeing the big picture (with small device)," Optics and Photonics News, no. 20.5, pp. 28-34, 2009.

[121] "Laser safety," Wikipedia. [Online]. Available: https://en.wikipedia.org/wiki/Laser_safety \#cite_note-iec-2060825-1-3.

[122] V. Milanovic, A. Kasturi, and V. Hachtel, "High brightness MEMS mirror based head-up display (HUD) modules with wireless data streaming capability," in Proc. of SPIE, 2015, vol. 9375 , p. 93750A.

[123] F. Chao, "Development of a micromirror based laser vector scanning automotive HUD," Ryerson University, 2011.

[124] J. Christmas, D. Masiyano, and N. Collings, "Holographic automotive head up displays," Milton Keynes, 2015.

[125] C.-P. Weng and G.-D. J. Su, "Using micro-projectors to realize large screen head-up display," in Proc. of SPIE, 2012, vol. 8486, no. 1, p. 84860I.

[126] "Head-up Displays - Eying safety and comfort at all times," Continental AG. [Online].

Available: http://www.continentalautomotive.com/www/automotive_de_en/themes/passenger_ca rs/interior/instrumentation_displays/pi_head_up_en.html?page $=3$.

[127] "Augmented Reality Head-up Display," Continental Automotive GmbH. [Online]. Available: http://continental-head-up-display.com/\#head-up.

[128] Benjamin Lee, "DMD 101: Introduction to Digital Micromirror Device ( DMD )," 
DLPA008A, 2013.

[129] A. Georgiou, J. Christmas, J. Moore, et al., "Liquid crystal over silicon device characteristics for holographic projection of high-definition television images.," Appl. Opt., vol. 47, no. 26, pp. 4793-4803, 2008.

[130] N. Collings, M. Reufer, R. V. Penty, et al., "Holographic projection based on tapered lasers and nematic liquid crystal on silicon devices," in Proc. of SPIE, 2010, vol. 7775, p. 777504.

[131] N. Collings, T. Davey, J. Christmas, D. Chu, and B. Crossland, "The applications and technology of phase-only liquid crystal on silicon devices," J. Disp. Technol., vol. 7, no. 3, pp. 112-119, 2011.

[132] H. Peng, D. Cheng, J. Han, et al., "Design and fabrication of a holographic head-up display with asymmetric field of view," Appl. Opt., vol. 53, no. 29, p. H177, 2014.

[133] "HUD in Land Rover Evoque," Land Rover. [Online]. Available: http://www.landrover.com.au/vehicles/range-rover-evoque/options-and-accessories.html.

[134] “TOLED automotive HUD.” [Online]. Available: http://www.glrhud.com/.

[135] M. K. Hedili, M. O. Freeman, and H. Urey, "Microlens array-based high-gain screen design for direct projection head-up displays.," Appl. Opt., vol. 52, no. 6, pp. 1351-7, 2013.

[136] J. Lincoln, D. Lashmet, and M. Carmean, "How a laser HUD can make driving safer," Redmond, WA, DA0119853, 2007.

[137] K. V Chellappan, E. Erden, and H. Urey, "Laser-based displays: a review," Appl. Opt., vol. 49, no. 25, pp. F79-98, 2010.

[138] K. Yamamoto, "Laser display technologies and their applications," Adv. Opt. Technol., vol. 1, no. 6, pp. 483-488, 2012.

[139] M. O. Freeman, "MEMS scanned laser head-Up display," in Proc. of SPIE, 2011, vol. 7930, p. $79300 \mathrm{G}$.

[140] M. K. Hedili, M. O. Freeman, and H. Urey, "Microstructured head-up display screen for automotive applications," in Proc. of SPIE, 2012, vol. 8428, p. 84280X.

[141] Y. Sun, S. Wu, and I. Spence, "The commingled division of visual attention," PLoS One, 
vol. 10 , no. 6 , pp. 1-18, 2015.

[142] Integrated Engineering Software Sales Inc., "Electro: Tow-Dimensional/RotationalSymmetrical Electrostatic Field Solver.” 2007.

[143] Coventor Inc., “Coventorware.” 2014.

[144] B. Borovic, a Q. Liu, D. Popa, H. Cai, and F. L. Lewis, “Open-loop versus closed-loop control of MEMS devices: choices and issues," J. Micromechanics Microengineering, vol. 15, no. 10, pp. 1917-1924, 2005.

[145] "MgF2 Coated Double-Concave (DCV) Lenses," Edmund Optics Inc., 2014. [Online]. Av ailable: http://www.edmundoptics.com/optics/optical-lenses/double-concave-dcv-spherical -singlet-lenses/commercial-grade-mgf2-double-concave-dcv-lenses/3496/.

[146] “Continental HUD viewing angle," Continental AG. [Online]. Available: http://automotive -eetimes.com/en/a-closer-view-to-continental-s-ar-hud.html?cmp_id=71\&news_id=22290 $3745 \&$ page $=1$. 